\title{
Surface plasmon-polariton Schottky photodetectors
}

\author{
by
}

SeyedMohammad Alavirad, M.A.Sc.

A thesis submitted to the Faculty of Graduate and Postdoctoral Affairs in partial fulfillment of the requirements for the degree of

Doctor of Philosophy

in

Electrical Engineering

Ottawa-Carleton Institute for Electrical and Computer Engineering

Carleton University

Ottawa, Ontario

(C) 2017

SeyedMohammad Alavirad 


\begin{abstract}
This article thesis presents the full design, fabrication, characterization and testing of two surface plasmon-polaritons (SPPs) photodetectors operating at telecommunication wavelengths.

Surface plasmon photodetectors are of great current interest. Such detectors typically combine a metallic structure that supports surface plasmons with a photodetection structure based on internal photoemission or electron-hole pair creation. We present two metal nano-structures supporting SPPs, integrated into a silicon-based Schottky-contact photodetector: an array of nanoantennas and nano-gratings. In both structures, incident photons coupled to the array excite SPPs on the gold $(\mathrm{Au})$ nanowires of the antennas which decay by creating "hot" carriers in the metal. The hot carriers may then be injected over the potential barrier at the Au-Si interface resulting in a photocurrent.
\end{abstract}

A metal grating is used to couple perpendicularly-incident light to SPPs propagating along a thin metal patch forming a Schottky contact to $\mathrm{p}$-Si. The responsivity is enhanced by the absorption of SPPs directly along the Schottky contact leading to the creation of hot holes near the contact. Our measurements reveal a high responsivity of $\sim 13 \mathrm{~mA} / \mathrm{W}$ at telecom wavelengths $(\sim 1550 \mathrm{~nm})$ and under low reverse bias. The responsivity increases with reverse bias. The optical bandwidth (FWHM) is $\sim 80 \mathrm{~nm}$. These detectors have many important advantages such as speed, simplicity, compatibility with silicon, and low-cost fabrication.

In the nanoantenna case, high responsivities of $100 \mathrm{~mA} / \mathrm{W}$ and practical minimum detectable powers of $-12 \mathrm{dBm}$ should be achievable near $1550 \mathrm{~nm}$ which makes monitoring of the spectral response very easy. The device was then investigated for use as a biosensor by computing its bulk and surface sensitivities. Sensitivities of $\sim 250 \mathrm{~nm} / \mathrm{RIU}$ (bulk) and $\sim 8 \mathrm{~nm} / \mathrm{nm}$ (surface) in water are predicted. We identify the mode propagating and resonating along the nanowires of the 
antennas, we apply a transmission line model to describe the performance of the antennas, and we extract two useful formulas to predict their bulk and surface sensitivities. As a biosensor, this structure offers significant advantages as it simplifies the interrogation setup: only the photocurrent generated by the device needs to be monitored, and the excitation optics (bottom) is physically separated from the micro-fluidics (top) by the device. We also propose a Schottky contact photodetector based on electrically-contacted $\mathrm{Au}$ nanoantennas on $\mathrm{p}$-Si for the plasmonic detection of sub-bandgap photons in the optical communications wavelength range. This highly compact component is very promising for applications in high-density Si photonic integration. 


\section{Acknowledgements}

Foremost I would like to thank my supervisors Dr. Pierre Berini and Dr. Langis Roy for their expertise, support, encouragement and most importantly their friendship over these many years. They have helped me to develop my research skills over the years we worked together. They have provided fantastic guidance and cooperation throughout my $\mathrm{PhD}$ studies.

I would like to acknowledge Anthony Olivieri, the optical and fabrication lab technician at Centre for Research in Photonics at the University of Ottawa who always assisted me in fabrication my samples and other invaluable guidance during the experimental measurements; I have always appreciated your patience and willingness to help without reservation.

Sincere thanks also to Saba S. Mousavi for her help with countless little things that allowed me to complete this one large thing. I am also grateful to Natural Sciences and Engineering Research Council of Canada (NSERC) and to Test Photonics Canada for their financial supports.

I would like to thank my best friend forever, Bijan Borzou, whom I cannot call anything but my brother. His help, presence and dedications in the last ten years of my life cannot be described by words. Without him being there, passing this long road was nothing but impossible

My parents have been essential in helping me achieve our dreams. I am blessed beyond measure to have parents whose support and encouragement, during the lowest of lows and the highest of highs, fuels me every moment. I would especially like to thank my mother for opening my eyes toward the dreams and for all her dedications for my life, my studies and my goals. Also, I have been so gifted for having a great father who has always been there for support. I like to appreciate the help and assistance of my brothers Hossein and Hesam in all these hard years of being far away from home.

Finally, and most importantly, I would like to thank my wife, Hoda. Her support, encouragement, quiet patience and unwavering love were undeniably the bedrock upon which the past few years of my life have been built.

I dedicate this work to my parents and my wife. 


\section{Table of Contents}

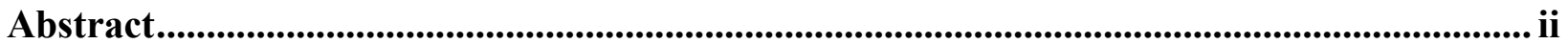

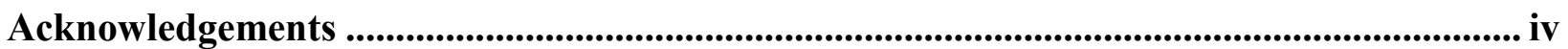

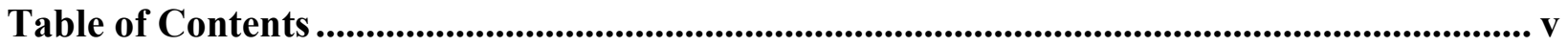

List of Tables ................................................................................................................................. vii

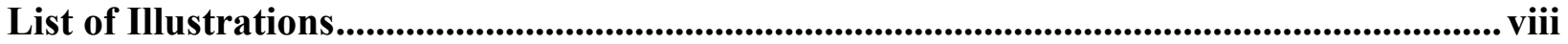

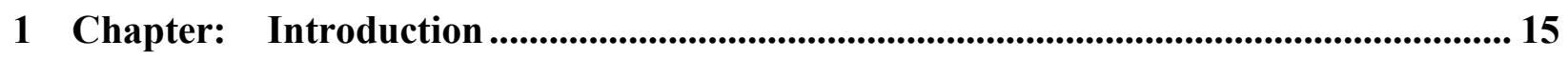

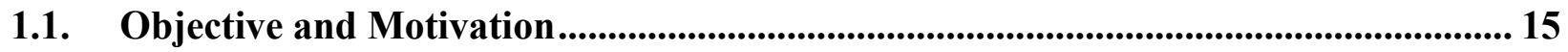

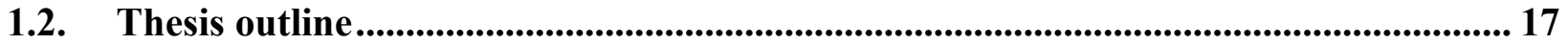

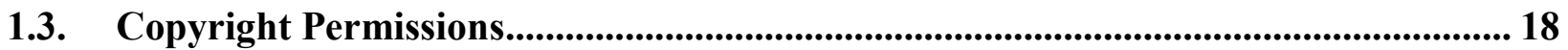

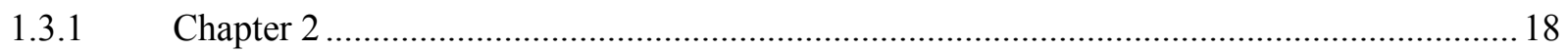

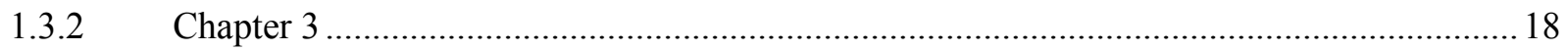

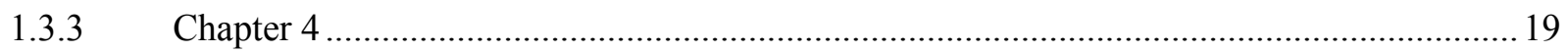

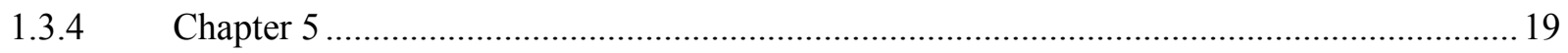

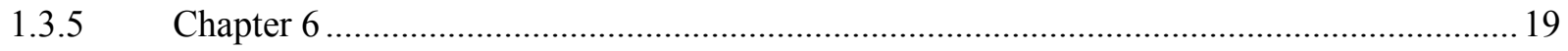

2 Chapter: Background and Literature Review ................................................................. 20

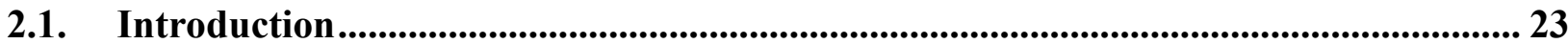

2.2. Operating Principles of IPE-based Devices.................................................................... 25

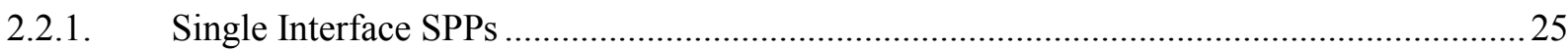

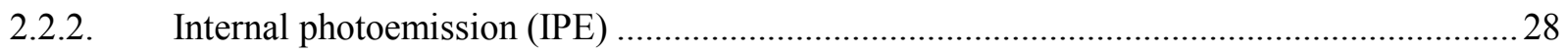

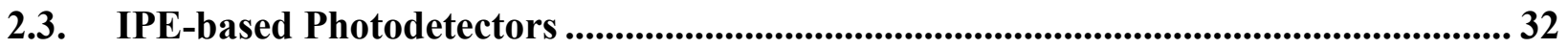

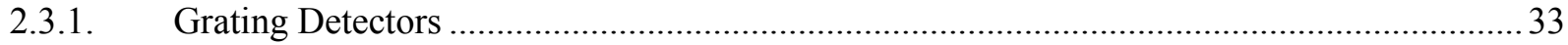

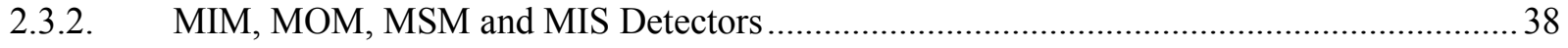

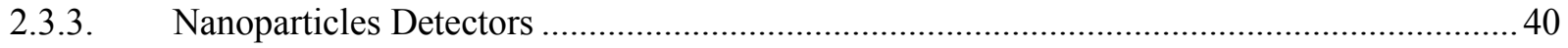




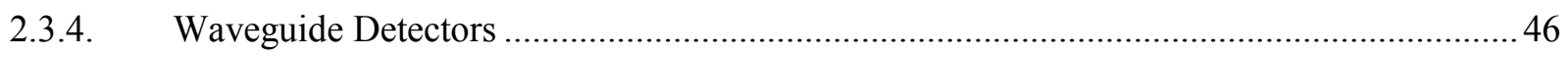

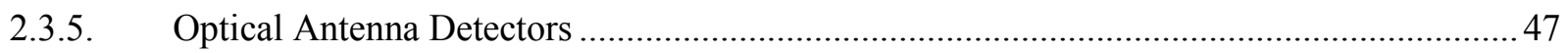

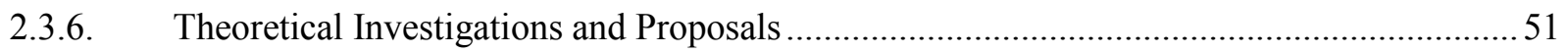

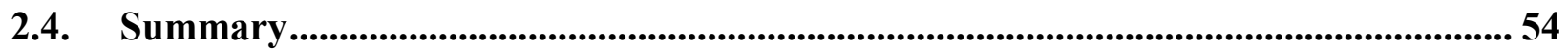

3 Chapter: Design, Fabrication and Measurement of a High-Responsivity Sub-Bandgap Hot-Hole Plasmonic Schottky Detectors ...........................................................................5 59

4 Chapter: Design a High-Sensitivity Schottky-Contact Plasmonic Nanoantenna ......... 73

5 Chapter: Optimization of Plasmonic Nanoantennas for Sensing Applications:

Consideration of Materials and Wavelengths ............................................................. 95

6 Chapter: Sub-Bandgap Photodetection with Electrically-Vontacted Plasmonic Nanoantennas on Silicon ............................................................................................................................. 104

7 Chapter: Conclusions and Future Work ............................................................. 109

7.1. Concluding remarks ......................................................................................................... 109

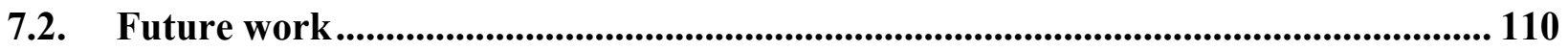

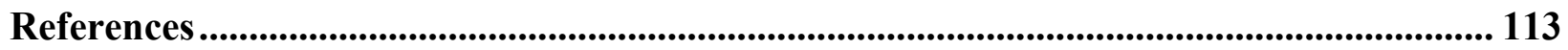




\section{List of Tables}

Table 1 Characteristics of some grating-based photodetectors 55

Table 2 Characteristics of some MIM and MSM photodetectors 56

Table 3 Characteristics of some nanoparticles-based photodetectors 56

Table 4 Characteristics of some waveguide-based photodetectors 57

Table 5 Characteristics of some nanoantennas-based photodetectors 57 


\section{List of Illustrations}

Illustration 1 (Adapted from [35]) Single-interface SPP and waveguide. The relative permittivity of the metal is denoted $\varepsilon_{\mathrm{r}, \mathrm{m}}$ and that of the dielectric $\varepsilon_{\mathrm{r}, \mathrm{d}}$. The distribution of the main transverse electric field component $\left(E_{\mathrm{y}}\right)$ of the SPP is sketched in blue on the structure

Illustration 2 (Adapted from [35]) Dispersion curve of the SPP along a $\mathrm{Ag} / \mathrm{SiO} 2$ semi-infinite interface; the light line in $\mathrm{SiO}_{2}$ is plotted as the green dash-dot curve

Illustration 3 (Adapted from [32]) (a) Schottky diode on n-Si illuminated by light of photon energy $h v$ (green). Reverse biasing $\left(V_{\mathrm{b}}<0\right)$ is assumed. Electrons are shown by filled dark blue circles and holes by unfilled ones. (b) Energy band diagram of a Schottky contact on n-Si and the 3 -step internal photoemission process consisting of $p$ - photoexcitation, $t$-transport, $e$ - emission. $E_{\mathrm{C}}$ and $E_{\mathrm{V}}$ are the conduction and valence band edges, respectively, $E_{\mathrm{F}}$ is the Fermi level, and $\Phi_{\mathrm{B}}$ is the Schottky barrier height

Illustration 4 (a) (Adapted from [31,48]) Photoexcitation processes following the illumination of a metal nanoparticle with a femtosecond laser pulse; the excitation of a localized surface plasmon redirects the flow of light (Poynting vector) towards and into the nanoparticle, (b) (Adapted from [46]) Simulation of field enhancement in a plasmonic bow-tie nanoantenna and the resulting electron trajectories

Illustration 5 (Adapted from [62]) (left) Structure of Au/Si Schottky-type SPP detector with a nanoslit grating. (right) Slit pitch dependencies on SPP intensity and photocurrent for ring type grating when slit pitch varies in the range of $440 \mathrm{~nm}$ to $1280 \mathrm{~nm}$

Illustration 6 (Adapted from [4]) Scanning electron micrographs (SEMs) of (a) a device used to evaluate the photoresponse measured by the devices with diffraction structures, (b) the multi-slit structure, (c) the disk array structure, and (d) no diffraction structure in place, (e) Photoresponses of the devices with each of the diffraction structures, (f) Polarization angle dependence of the photocurrent detected by the device with the multi-slit structure

Illustration 7 (Adapted from [27] (a) Schematic of a gold grating on an n-type Si with a 2-nm Ti adhesion layer, oriented transverse to the laser polarization. The polarization of the incident beam and its $k$ vector are represented by yellow and green arrows, respectively, (b) SEM image of a gold grating structure with a grating thickness $(T)=200 \mathrm{~nm}$, interslit distance $(D)=950 \mathrm{~nm}$ and slit width $(W)=250 \mathrm{~nm}$. For all structures the array measured $12 \times 12 \mu \mathrm{m}$. The scale bar is $1 \mu \mathrm{m}$. (c) Responsivities of grating-based photodetectors for three different gold layer thicknesses, $T=93$ $\mathrm{nm}$ (black), $170 \mathrm{~nm}$ (grey) and $200 \mathrm{~nm}$ (green), showing a strong intensity dependence on grating thickness

Illustration 8 (Adapted from [63]) (a) Scanning electron micrographs of an uncoated $\mathrm{Au} / p-\mathrm{Si}$ nanograting photodetector. The pitch of the device is measured of $406 \mathrm{~nm}$ (400 nm designed) and the thickness of the Au patch is measured as $29.8 \mathrm{~nm}$ ( $20 \mathrm{~nm}$ designed) and its duty cycle of this is $60 \%$, (b) Measured photocurrent response of three grating photodetectors; $V_{\mathrm{B}}=-100 \mathrm{mV}, \Lambda=$ 390,400 and $410 \mathrm{~nm}$, duty cycle of $62 \%$ and patch diameter of $25 \mu \mathrm{m}$, (c) Photocurrent generated 
by grating detectors $v s$. incident power $P_{\text {inc }}$ measured for three different pitches. A linear fit is applied to the data and the slope corresponds to the responsivity of the device

Illustration 9 (Adapted from [67]) (a) Schematic illustration of a typical device featuring a series of MIM crossbar junctions formed between a wide bottom electrode and a set of nanoscale top electrodes. The directions of the electric field for transverse electric (TE) and transverse magnetic (TM) illumination are as depicted. (b) SEM image of a device with a $470 \mathrm{~nm}$ wide top electrode. The inset shows a cross-sectional image of the device, composed of an oxide layer sandwiched by top and bottom metal layers. (c) Photocurrent image of the device taken at an illumination wavelength of $400 \mathrm{~nm}$ with TM polarization overlaid on the SEM image of the device. The red color corresponds to $7 \mathrm{pA}$ and blue indicates a close to zero photocurrent

Illustration 10 (Adapted from [69]) (a) Schematic of the proposed photodetector integrated with SOI waveguide, (b) Responsivity vs reverse voltage applied at $1550 \mathrm{~nm}$

Illustration 11 (Adapted from [73]) (left) High-resolution transmission electron microscopy (HRTEM) images of the cross-section at the interface of the Au-NIs and the $\mathrm{TiO}_{2}$ annealed at $800^{\circ} \mathrm{C}$, (right) The incident photocurrent efficiency (IPCE) action spectrum measured with the Au$\mathrm{NIs} / \mathrm{TiO}_{2}$ photoelectrode (red) and the extinction spectrum of the $\mathrm{Au}-\mathrm{NIs} / \mathrm{TiO}_{2}$ photoelectrode (black). The applied potential was $0.3 \mathrm{~V}$ versus a saturated calomel electrode (SCE)

Illustration 12 (Adapted from [77]) SEM of Ag nanoparticles in $\mathrm{TiO}_{2}$ nanotubes

Illustration 13 (Adapted from [79]) SEM of Ag on $\mathrm{ZnO}$ flower-rod heterostructures

Illustration 14 (Adapted from [56]) (a) Depiction of broad-band NIR Si Schottky photodetector. $\mathrm{Au}$ nanoislands are formed on n-type Si by rapid thermal annealing of a thin Au film. Si substrate is used as the bottom contact; and Au nanoislands together with the AZO capping layer constitute the top contact, (b) SEM image of the samples annealed at $450^{\circ} \mathrm{C}$

Illustration 15 (Adapted from [80]) Oxidation of Ag nanorods and redeposition of small Ag nanoparticles takes place as a result of plasmon-induced charge separation. After LSPR excitation and hot-electron generation, the oxidized $\mathrm{Ag}^{+}$ions diffuse in the water layer adsorbed on the $\mathrm{TiO}_{2}$ substrate and eventually recombine with electrons from $\mathrm{TiO}_{2}$, forming satellite redeposited islands. The AFM images show Ag nanorods with various aspect ratios (ARs) that have undergone excitation of multipole plasmon modes from $m=1$ to 4 after irradiation with $800 \mathrm{~nm}$. The rods experience changes in length depending on the incident wavelength and mode excited. The horizontal scale bars are $50 \mathrm{~nm}$ in length

Illustration 16 (Adapted from [50]) Sketch of an SPP waveguide detector consisting of a metal stripe forming a Schottky contact on $\mathrm{Si}$, excited in the $s a_{\mathrm{b}}{ }^{0}$ mode [82] via butt-coupling to a tapered polarisation-maintaining single mode fibre (PM-SMF). (b) (Adapted from [50]) Spectral response of a $\mathrm{Au}$ on $\mathrm{n}-\mathrm{Si}$ detector as sketched in (a), plotted in Fowler form. The Au stripe width and thickness were $2.5 \mu \mathrm{m}$ and $135 \mathrm{~nm}$, the reverse bias was $200 \mathrm{mV}$ and the incident optical power was $2 \mathrm{~mW}$. (c) (Adapted from [6].) Photocurrent map ( $\mu \mathrm{A})$ of a Au on p-Si detector as sketched in (a), generated by scanning a tapered PM-SMF over the end facet using a piezoelectric nanopositioner. The Au stripe width and thickness were $1.5 \mu \mathrm{m}$ and $40 \mathrm{~nm}$ (sketched as the black 
horizontal line), the reverse bias was $100 \mathrm{mV}$, the incident optical power was $1 \mathrm{~mW}$, and the excitation wavelength was $1310 \mathrm{~nm}$

Illustration 17 (Adapted from [55]) SEM images of a plasmonic enhanced Si pyramids, (a) Formation of the nanoapex in the silicon pyramid. (b) SEM image of a final fabricated device, (c) $I-V$ measurements of the pyramid Schottky device at constant optical power for three different wavelengths and dark measurement. The inset shows the photocurrent versus optical power data and linear fit for $1550 \mathrm{~nm}$ wavelength

Illustration 18 (Adapted from [87]) (a) Schematic of the CPL detector consisting of a chiral metamaterial integrated with a semiconductor that serves as a hot electron acceptor. The Ohmic contact on $\mathrm{Si}$ is formed by soldering indium. The circuit is formed by wire bonding to the silver bus bar and indium, (b), (c) Photoresponsivity spectra of experimentally measured (dots) and theoretically calculated (solid curve) under LCP (blue) and RCP (red) illumination for LH (b) and $\mathrm{RH}(\mathrm{c})$ metamaterials

Illustration 19 (Adapted from [91]) (a) Three-dimensional schematic diagram of the proposed $\mathrm{Si}$ photodetector, (b) Emission cones with angle, $\Omega$ : (top-left) a momentum-space illustration; (topright) real-space illustrations for round $\mathrm{Au}-\mathrm{Si}$ interfaces and (bottoms) an asymmetric corner denoted by the red dashed rectangles when (left) $\Omega \geq \pi / 4$ and (right) $\Omega<\pi / 4$, (c)responsivity, R, for both TE (solid lines) and TM (dotted lines) polarizations, and (d) polarization dependent deviation PDD of responsivity, for photodetectors with $h_{\mathrm{Au}}=10,20,30,50,70$, and $90 \mathrm{~nm}$. The SU8 film thickness is $h_{\mathrm{SU}}=60 \mathrm{~nm}$

\section{Chapter 3}

Illustration 1 (a) Cross-sectional sketch of the proposed $\mathrm{Au} / \mathrm{Si}$ Schottky surface plasmon photodetector. The structure comprises a metal patch of thickness $t$ on $\mathrm{p}-\mathrm{Si}$, with a metal grating consisting of rectangular ridges of width $W$ and thickness $H$ arranged periodically in pitch $\Lambda$. The materials considered are $\mathrm{Au}$ for the metal, $\mathrm{p}-\mathrm{Si}$ for the epitaxial layer and $\mathrm{p}^{+}-\mathrm{Si}$ for the substrate. The device is illuminated from the top with polarised infrared light perpendicular to the grating. (b) Energy band diagram of a metal contact to a p-type semiconductor and the 3-steps of the internal photoemission process: $p$ : photoexcitation, $t$ : transport, $e$ : emission. $E_{\mathrm{C}}$ and $E_{\mathrm{V}}$ are the conduction and valence band edges, respectively, $E_{\mathrm{F}}$ is the Fermi level, and $\Phi_{\mathrm{B}}$ is the Schottky barrier height. (c) Hot hole photoemission across the Schottky junction between a metal and a ptype semiconductor

Illustration 2 Power ratios (absorptance $A_{0}$; transmittance $T_{0}$; reflectance $R_{0}$; and coupling coefficient $C_{0}$ ) of grating photodetectors operating near $1550 \mathrm{~nm}$, with a duty cycle of $60 \%$ and $t$ $=20 \mathrm{~nm}$ (solid lines), and a duty cycle of $62 \%$ and $t=28.9 \mathrm{~nm}$ (dashed lines) for grating designs having $W=200 \mathrm{~nm}, H=80 \mathrm{~nm}$ and (a) $\Lambda=390 \mathrm{~nm}$, (b) $\Lambda=400 \mathrm{~nm}$, and (c) $\Lambda=410 \mathrm{~nm}$. The resonance red-shifts with increasing pitch. Electric field distribution of the gratings corresponding to $\Lambda=400 \mathrm{~nm}, t=20 \mathrm{~nm}$, computed on resonance $\left(\lambda_{0}=1560 \mathrm{~nm}\right) ;(d) \operatorname{Re}\left\{E_{\mathrm{x}}\right\},(\mathrm{e}) \operatorname{Re}\left\{E_{\mathrm{z}}\right\}$ and (f) $|\boldsymbol{E}|$ (also see Visualization 1). The incident electric field strength was set to $1 \mathrm{~V} / \mathrm{m}$

Illustration 3 (a), (b) SEM and AFM images, respectively, of an uncoated Au/p-Si grating photodetector. The pitch of the device is measured as $406 \mathrm{~nm}$ ( $400 \mathrm{~nm}$ designed) and the thickness of the Au patch is measured as $28.9 \mathrm{~nm}$ (20 nm designed). The duty cycle of this device is $60 \%$. 
(c) Optical microscope image of photodetectors of varying grating period, e-beam energy dose factors and duty cycles. Each color spot corresponds to one detector. The area shown is about 8 $\mathrm{mm}^{2}$ and contains $\sim 170$ detectors

Illustration 4 (a) The orange curve shows an average of five dark $I-V$ characteristics for a grating photodetector of diameter $28 \mu \mathrm{m}$ and $\Lambda=400 \mathrm{~nm}$. The purple curve is obtained after removing the effects of the shunt and series resistors shown in the non-ideal model in inset. (b) Measured photocurrent response of three grating photodetectors; $V_{\mathrm{B}}=-100 \mathrm{mV}, \Lambda=390,400$ and $410 \mathrm{~nm}$, duty cycle of $62 \%$ and patch diameter of $25 \mu \mathrm{m}$. A 4-period moving average is plotted on each response as the bold curve. The rapid wavelength variations correspond to Fabry-Perot resonances - the upper right inset is an enlarged response showing such resonances and the lower right inset shows a response calculated using the TMM method, (c) Deep traps formed by the diffusion of $\mathrm{Au}$ into $p$-Si

Illustration 5 (a) Photocurrent generated by grating detectors $v s$. incident power $P_{\text {inc,d }}$ measured for three different pitches: $\Lambda=390 \mathrm{~nm}\left(\lambda_{01}=1537 \mathrm{~nm}\right), \Lambda=400 \mathrm{~nm}\left(\lambda_{02}=1548 \mathrm{~nm}\right)$ and $\Lambda=410$ $\mathrm{nm}\left(\lambda_{03}=1570 \mathrm{~nm}\right)$. A linear fit is applied to the data and the slope corresponds to the responsivity of the device. (b) Responsivity $v s$. reverse bias measured for the photodetector with $\Lambda=400 \mathrm{~nm}$ at $\lambda_{0}=1548 \mathrm{~nm}$; the dashed curve shows a trend curve for the responsivity obtained by taking into account the Schottky barrier lowering effect

\section{Chapter 4}

Illustration 1 (a) Array of $\mathrm{Au}$ dipoles on $p$-Si on $p^{+}$-Si covered by $\mathrm{H}_{2} \mathrm{O}$. Al Ohmic contacts are located at the bottom of the structure and an Au circular contact pad is connected to all dipole arms via optically non-invasive perpendicular Au interconnects. A plane wave source illuminates the array in the $z$-direction from below. (b) Geometry of a unit cell of the array under study (interconnects are shown as well); the dipole is assumed covered by an adlayer of thickness $a$ when the surface sensitivities are computed

Illustration 2 Distribution of $|\boldsymbol{E}|=\sqrt{\left|\boldsymbol{E}_{\boldsymbol{x}}\right|^{2}+\left|\boldsymbol{E}_{\boldsymbol{y}}\right|^{2}+\left|\boldsymbol{E}_{\boldsymbol{z}}\right|^{2}}$ on $x-y$ cross-sectional planes for an Au dipole of dimensions $w=30 \mathrm{~nm}, t=30 \mathrm{~nm}, l=210 \mathrm{~nm}, g=20 \mathrm{~nm}$ and $p=q=300 \mathrm{~nm}$ (a) 3 $\mathrm{nm}$ above the $\mathrm{Au} / \mathrm{Si}$ interface, (b) $15 \mathrm{~nm}$ above the $\mathrm{Au} / \mathrm{Si}$ interface, and (c) $3 \mathrm{~nm}$ above the $\mathrm{Au}$ surface in $\mathrm{H}_{2} \mathrm{O}$. Computations performed at $\lambda_{0}=1353 \mathrm{~nm}$ (resonance wavelength)

Illustration 3 Calculated transmittance $(T)$, reflectance $(R)$, absorptance $(A)$ and electric field enhancement $\left(E_{e n}\right) v s$. free space wavelength $\left(\lambda_{0}\right)$

Illustration 4 (a) Internal quantum efficiency $\left(\eta_{i}^{t}\right) v s . \lambda_{0}$. (b) Responsivity $\left(R_{e s p}\right)$ and minimum detectable power $\left(S_{\min }\right) v s . \lambda_{0}$

Illustration 5 (a) Absorptance $(A) v s$. $\lambda_{0}$ for several cover refractive indices $n_{c}$ ranging from 1 to 2.75). (b) Bulk sensitivity $\left(\partial \lambda_{0 r} / \partial n_{c}\right.$ - blue) and peak responsivity ( $R_{e s p, r}$ - red) of the rectenna as a function of $n_{c}$

Illustration 6 Real part of $E_{z}$ of the $s a_{b}^{0}$ mode plotted over the cross-section of a nanowire waveguide $\left(\lambda_{0}=1353 \mathrm{~nm}\right)$ computed using a mode solver. (a) $n_{c}=1$ (air) and (b) $n_{c}=2.75 \quad 84$ 
Illustration 7 Effective refractive index ( $n_{\text {eff }}-$ blue) and mode power attenuation $(\alpha$-red) of the $s a_{b}^{0}$ mode resonating along the dipoles as a function of $n_{\mathrm{c}}$

Illustration 8 Gap capacitance $C_{g}$ (blue) and characteristic impedance $Z_{0}$ of the $s a_{b}^{0}$ mode (red) as a function of $n_{c}$

Illustration 9 (a) Resonant wavelengths computed using the transmission line model and the FDTD method as a function of bulk index $n_{c}$. (b) Bulk sensitivity computed using the FDTD method (dashed blue), the transmission line model (Eq. (4) - red), and the analytical solution (Eq. (20) - black)

Illustration 10 (a) Absorptance $A v s$. $\lambda_{0}$ for several adlayer thicknesses $(a=0$ to $5 \mathrm{~nm}$ ); the curves are offset vertically by -0.05 for clarity. (b) Surface sensitivity $\left(\partial \lambda_{0 r} / \partial a-\right.$ blue) and peak responsivity $\left(R_{e s p, r}-\right.$ red $)$ as a function of $a$

Illustration 11 Real part of $E_{z}$ of the $s a_{b}^{0}$ mode plotted over the cross-section of a nanowire waveguide ( $\lambda_{0}=1353 \mathrm{~nm}$ ) computed using a mode solver. (a) $a=0$ (no adlyaer) and (b) $a=5 \mathrm{~nm}$

Illustration 12 Effective refractive index ( $n_{\text {eff }}-$ blue) and mode power attenuation ( $\alpha$ - red) of the $s a_{b}^{0}$ mode resonating along the dipoles as a function of $a$

Illustration 13 Schematic of a dipole gap showing three plate capacitances in series

Illustration 14 Gap capacitance $C_{g}$ (blue) and characteristic impedance $Z_{0}$ of the $s a_{b}^{0}$ mode (red) as a function of $a$

Illustration 15 (a) Resonant wavelengths computed using the transmission line model and the FDTD method as a function of adlayer thickness $a$. (b) Surface sensitivity computed using the FDTD method (dashed blue), the transmission line model (Eq. (4) - red), and the analytical solution (Eq. (29) - black)

\section{Chapter 5}

Illustration 1 Array of Au nanodipoles on silicon or glass covered by water; a plane wave source illuminates the array in the $z$-direction from the top or bottom

Illustration 2 Transmission line model of a dipole antenna where the gap is modeled as a parallelplate capacitor [20]

Illustration 3 Modal parameters, bulk sensitivity and length for an array of nanodipoles covered by water on silicon designed for $\lambda_{0 d}=850 \mathrm{~nm} v s$. thickness (or gap), swept over width with $g=20$ $\mathrm{nm}$, pitch size of $300 \mathrm{~nm} \times 300 \mathrm{~nm}$; top: (left) characteristic impedance, (middle) effective refractive index, (right) capacitance of the gap, bottom: (left) mode power attenuation, (middle) bulk sensitivity and nanodipole length, (right) bulk sensitivity and nanodipole length swept over $g(t=35$ $\mathrm{nm})$ 
Illustration 4 Modal parameters, bulk sensitivity and length for an array of nanodipoles covered by water on silicon designed for working at $\lambda_{0 d}=1310 \mathrm{~nm} v s$. thickness (or gap) swept over width with $g=20 \mathrm{~nm}$, pitch size of $300 \mathrm{~nm} \times 300 \mathrm{~nm}$; top: (left) characteristic impedance, (middle) effective refractive index, (right) capacitance related to the gap region, bottom: (left) mode power attenuation, (middle) bulk sensitivity and nanodipole length, (right) bulk sensitivity and nanodipole length swept over $g(t=35 \mathrm{~nm})$

Illustration 5 Modal parameters, bulk sensitivity and length for an array of nanodipoles covered by water on silicon designed for working at $\lambda_{0 d}=1550 \mathrm{~nm} v s$. thickness (or gap) swept over width with $g=20 \mathrm{~nm}$, pitch size of $360 \mathrm{~nm} \times 360 \mathrm{~nm}$; top: (left) characteristic impedance, (middle) effective refractive index, (right) capacitance related to the gap region, bottom: (left) mode power attenuation, (middle) bulk sensitivity and nanodipole length, (right) bulk sensitivity and nanodipole length swept over $g(t=35 \mathrm{~nm})$

Illustration 6 Modal parameters, bulk sensitivity and length for an array of nanodipoles covered by water on glass designed for working at $\lambda_{0 d}=850 \mathrm{~nm} v s$. thickness (or gap) swept over width with $g=20 \mathrm{~nm}$, pitch size of $300 \mathrm{~nm} \times 300 \mathrm{~nm}$; top: (left) characteristic impedance, (middle) effective refractive index, (right) capacitance related to the gap region, bottom: (left) mode power attenuation, (middle) bulk sensitivity and nanodipole length, (right) bulk sensitivity and nanodipole length swept over $g(t=35 \mathrm{~nm})$

Illustration 7 Real part of $E_{z}$ of the $s a^{0}{ }_{b}$ mode plotted over the cross-section of a nanodipole arm $\left(\lambda_{0 d}=850 \mathrm{~nm}\right)$ computed using a mode solver; top: glass and bottom: silicon

101

Illustration 8 Modal parameters, bulk sensitivity and length for an array of nanodipoles covered by water on glass designed for working at $\lambda_{0 d}=1310 \mathrm{~nm} v s$. thickness (or gap) swept over width with $g=20 \mathrm{~nm}$, pitch size of $440 \mathrm{~nm} \times 440 \mathrm{~nm}$; top: (left) characteristic impedance, (middle) effective refractive index, (right) capacitance related to the gap region, bottom: (left) mode power attenuation, (middle) bulk sensitivity and nanodipole length, (right) bulk sensitivity and nanodipole length swept over $g(t=35 \mathrm{~nm})$

Illustration 9 Modal parameters, bulk sensitivity and length for an array of nanodipoles covered by water on glass designed for working at $\lambda_{0 d}=1550 \mathrm{~nm} v s$. thickness (or gap) swept over width with $g=20 \mathrm{~nm}$, pitch size of $480 \mathrm{~nm} \times 480 \mathrm{~nm}$; top: (left) characteristic impedance, (middle) effective refractive index, (right) capacitance related to the gap region, bottom: (left) mode power attenuation, (middle) bulk sensitivity and nanodipole length, (right) bulk sensitivity and nanodipole length swept over $g(t=35 \mathrm{~nm})$

Illustration 10 Comparison of bulk sensitivity obtained from modal analysis (solid blue) to FDTD (green stars) on silicon: (left) $w=25 \mathrm{~nm}, \lambda_{0 d}=850 \mathrm{~nm}$, (middle) $w=10 \mathrm{~nm}, \lambda_{0 d}=1310 \mathrm{~nm}$, and (right) $w=10 \mathrm{~nm}, \lambda_{0 d}=1550 \mathrm{~nm}$

Illustration 11 Comparison of bulk sensitivity obtained from modal analysis (solid blue) to FDTD (green stars) on glass: (left) $w=10 \mathrm{~nm}, \lambda_{0 d}=850 \mathrm{~nm}$, (middle) $w=10 \mathrm{~nm}, \lambda_{0 d}=1310 \mathrm{~nm}$, and (right) $w=10 \mathrm{~nm}, \lambda_{0 d}=1550 \mathrm{~nm}$

\section{Chapter 6}


Illustration 1 Energy band diagram of a metal contact to a p-type semiconductor and the 3-steps of the internal photoemission process: $p$ : photoexcitation, $t$ : transport, $e$ : emission. $E_{\mathrm{C}}$ and $E_{\mathrm{V}}$ are the conduction and valence band edges, respectively, $E_{\mathrm{F}}$ is the Fermi level, and $\Phi_{\mathrm{B}}$ is the Schottky barrier height

106

Illustration 2 Schematic of an Au-Ti/Si optical nanoantenna Schottky-contact diode for subbandgap detection. The materials used are Au for the nanoantenna array, $\mathrm{p}$-Si for the epitaxial layer and $\mathrm{p}^{+}-\mathrm{Si}$ for the substrate and $\mathrm{Au} / \mathrm{Ti}$ for the probing structure (pad and arm). The device is illuminated from the top with polarised infrared light aligned to the nanomonopole lengths. A good design of length $125 \mathrm{~nm}$, width $44 \mathrm{~nm}$ and thickness $20 \mathrm{~nm}$ produces a resonant wavelength at $\lambda$ $=1534 \mathrm{~nm}$ as identified from the absorptance response in inset.

Illustration 3 SEM image of a representative nanoantenna array on p-Si: (top) overall view of the device consisting of a bilayer $\mathrm{Au} / \mathrm{Ti}$ probe structure, and (bottom) an array of Au electricallycontacted nanomonopole antennas.

Illustration 4 The black curve shows an average of five dark $I-V$ characteristics for complete device. The inset shows the dark $I-V$ characteristics of the probing structure only.

Illustration 5 Measured photocurrent response (left panels) of two electrically-contacted nanomonopole array photodetectors $\left(V_{\mathrm{B}}=-10 \mathrm{mV}\right)$. The nanomonopoles are $45 \mathrm{~nm}$ wide, $17 \mathrm{~nm}$ thick and $124 \mathrm{~nm}$ long (top panels) or $130 \mathrm{~nm}$ long (bottom panels). The rapid wavelength variations correspond to Fabry-Perot resonances. The corresponding photocurrent vs. incident power are also plotted (right panels); the slope of the linear fit gives the responsivity of each device. 


\section{Chapter: Introduction}

\subsection{Objective and Motivation}

Nanometallic structures are key to the conversion of light to surface plasmon-polaritons (SPPs) localized to ultra-small volumes. They are essential for several applications including photodetection and biosensing as they can provide highly enhanced fields, strong confinement (to sub-wavelength scale) and high bulk and surface sensitivities.

A Schottky barrier photodetector formed at the interface between a metal and a lightly doped semiconductor can be used to detect infrared radiation below the bandgap energy of the substrate via the internal photoelectric effect (IPE) [1,2]. Such detectors involving SPP excitations in prism [3], grating-coupled detectors [4, 5], waveguides [6, 7] and nanoparticles and nanoantennas $[8,9,10,11,12]$ have been reported; prism coupling to SPPs on a suitable detection structure was one of the first types of SPP detectors investigated in $[13,14,15]$. The Otto coupling configurations used in these studies has been more popular in experimental work, mostly because the detection structures can be fabricated independently, then the prism aligned to the detector in the set-up [16].

Schottky photodetectors based on IPE, integrated with a thin metal stripe waveguide in symmetric [17] and asymmetric [18] claddings have also been proposed, operating with long range and single-interface SPPs, respectively. Also, SPP metal stripe waveguides with asymmetric claddings were investigated for application in integrated Schottky photodetectors on Si [19].

A nanograting structure can be used as an alternative to a prism to increase the in-plane momentum of the incident light in order to match that of the SPP [20] providing compactness and manufacturing advantages and high detection sensitivity. A metal-semiconductor (Schottky) nanograting structure has been investigated to implement SPP photodetectors [21, 22]. They are 
sensitive to the angle of incidence, polarization and wavelength of the light. The SPP photodetector consists of a $\mathrm{Au} / \mathrm{Si}$ Schottky diode with a grating and the detector converts the SPP signals into electrical signals as photocurrents [23]. Because the responsivity of the SPP detector is closely related to the SPP intensity at the $\mathrm{Au} / \mathrm{Si}$ interface, the grating structure can be optimized in terms of the slit pitch and film thickness $[24,25]$. Absorption enhancement is therefore observed in the detection region as a result of incorporating nanogratings [26]. High responsivity is achievable in the presence of nanogratings [27], providing more generated photocurrent. They can also be used as means of increasing the speed of photodetection by lowering the detector capacitance and carrier transit time [28]. Nanograting structures investigated as SPP photodetectors include either an interrogated grating or an array of metal nanowires on a metal-oxide-metal structure, a metal semiconductor structure [29], a metal-oxide-semiconductor [30], or a commercial photodiode [31].

Small metal particles and antennas exhibit resonant responses under optical excitation, structure's shape, size and composition, the dielectric environment in which they find themselves, and the wavelength of illumination [32]. Also, the electric near-fields produced by antennas can be of much greater intensity than the incident electric fields, therefore photodetection applications are more convenient as it allows the detection volume to shrink, leading potentially to faster devices with compelling signal to noise characteristics.

Although many theoretical and experimental studies have been carried out on various aspects of nanogratings, nanoparticles and nanoantennas and their detection applications, achieving a sharp resonant peak (which is easier to track) is still a difficult challenge with most plasmonic nanostructures. Designing well-defined nanostructures, investigating their responsivities and sensitivities systematically, and verifying them experimentally could be done, where we obtain all spectral responses by measuring the photocurrent collected into the 
semiconductor. In this thesis, we deal with two different plasmonic nanostructures operating as Schottky barrier photodetectors: plasmonic nanoantenna arrays comprised of $\mathrm{Au}$ nanowires of rectangular cross-section and a plasmonic detectors of Au nanogratings. High responsivity, large sensitivity, operating wavelengths, small dimensions, easy measurement techniques to characterize them and lower fabrication costs using the most accessible materials are of their main advantages which we will discuss in more details later. In summary, this thesis covers the following topics:

- Design, fabrication and measurements of nanograting-based Schottky photodetectors operating at telecommunication wavelength, suitable for wafer probing applications;

- Design, fabrication and measurements of nanoantenna-based Schottky photodetectors suitable for detection applications.

\subsection{Thesis outline}

The dissertation material is presented in an integrated article format with each chapter being an aspublished or in-review scientific journal article with self-contained background/theory, discussion, references and page numbering. As a result, some of the material may be repeated among the articles, particularly introductory matter. The references for non-article materials can be found in the References section at the end of this document.

Chapter 2 presents background material and a comprehensive literature review on plasmonics, photodetectors and detection and sensing applications. Chapter 3 begins with first nanostructure proposed in this thesis, a nanograting coupler based photodetectors operating at telecommunication wavelengths. Chapter 4 deals with the second structure which is a nanoantenna array, it brings the design and application point of views. Chapter5 talk about optimization of 
nanoantenna photodetector proposed in chapter 3 for biosensing applications and suggests other type of materials can be used alternatively and finally chapter 6 present experimental results of nanoantenna photodetectors.

\subsection{Copyright Permissions}

\subsubsection{Chapter 2}

The article in this chapter is presented in full and is reprinted with the permission of SPIE Journal of Photonics for Energy (JPE).

[CH2] M. Alavirad, L. Roy and P. Berini, "Surface plasmon enhanced photodetectors based on internal photoemission," SPIE J. Photon. Energy 6(4), 042511 (2016).

https://doi.org/10.1117/1.JPE.6.042511

\subsubsection{Chapter 3}

The article in this chapter is presented in full and is reprinted with the permission of Optics Express.

[CH3] M. Alavirad, A. Olivieri, L. Roy and P. Berini, "High-responsivity sub-bandgap hot-hole plasmonic Schottky detectors," Opt. Express, 24(20), 22544-22554 (2016)

•https://doi.org/10.1364/OE.24.022544 


\subsubsection{Chapter 4}

The article in this chapter is presented in full and is reprinted with the permission of Optics Express.

[CH4] M. Alavirad, S. S. Mousavi, L. Roy and P. Berini, "Schottky-contact plasmonic dipole rectenna concept for biosensing," Opt. Express, 21(4), 4328-4347 (2013)

•https://doi.org/10.1364/OE.21.004328

\subsubsection{Chapter 5}

The article in this chapter is presented in full and is reprinted with the permission of IEEE Journal of Selected Topics in Quantum Electronics (IEEE JSTQE).

[CH5] M. Alavirad, L. Roy and P. Berini, “Optimization of Plasmonic Nanodipole Antenna Arrays for Sensing Applications," IEEE J. Sel. Top. Quant. 20(3), 7-14 (2014)

https://doi.org/10.1109/JSTQE.2013.2289963

\subsubsection{Chapter 6}

As of the publication of this thesis, this article has been prepared to be submitted to the Optics Letter.

[CH6] M. Alavirad, A. Olivieri, L. Roy and P. Berini, "Sub-bandgap photodetection with electrically-contacted plasmonic nano-antennas on silicon," to be submitted to Opt. Lett. (2016) 


\section{Chapter: Background and Literature Review}

The following article entitled "Surface plasmon enhanced photodetectors based on internal photoemission" was published in SPIE Journal of Photonics for Energy, Volume 6, Issue 4 on Oct. 2016 and is presented in full and in a plain format.

The article surveys the relevant literature comprehensively. It was performed solely by the primary author under supervision and guidance of the co-authors. In this review paper, authors report on the operating principles behind surface plasmon-polaritons photodetectors based on the internal photoelectric effect, and they survey and compare the most recent and leading edge concepts reported in the literature. This is significant and of great importance because the progress on SPP photodetectors has been rapid in the recent years, and interest on the topic continues to increase by the fact that it enables sub-bandgap detection so the optical bandwidth of a detector can be extended to photon energies below the bandgap of the semiconductor. This article prompts a review of most recent works in this area. It also highlights avenues that appear promising.

Reader of review paper in chapter 2 might need to know the background of Maxwell equations applied to a metal-semiconductor interface. Thereafter, the surface plasmon polaritons (SPPs) concepts are explained clearly but it worth that we discuss bases of surface plasmons (SPs) and difference between SPPs and SPs beforehand.

If we talk about a neutral plasma (which can be the free charges in a metal) and we are enabled to pull the negative and positive charges apart and let them go, they would oscillate due to the electrostatic potential. This is an excitation known as a plasma oscillation. A Plasmon is the quasiparticle associated with plasma oscillations (analogous to phonons being the quasiparticles

associated with mechanical vibrational modes). Surface plasmons (SPs) are plasmon excitations at the boundary of the material. Polaritons are quasiparticles that exist when an EM mode is 
strongly coupled to some other type of mode, i.e. if light strongly couples to some other physical excitation then the particle picture is inaccurate if we treat the 2 degrees of freedom separately, so instead of saying we have some photons and we have some particles representing the excitation, instead we are dealing with a hybrid particle that is simply called a polariton. Because there are lots of types of excitations, such particles are called [*] polaritons, where [*] is the name of the particles of the excitation.

Therefore, Surface plasmon polaritons (SPPs) are the quasiparticles of the coupled modes of an EM field and a surface plasma oscillation. Although SPs are not the same as SPPs, from the context the two are rarely confused. In addition, the charge oscillations will create an EM field, and so we cannot create an SP without it automatically being part of an SPP. And since "surface plasmon polaritons" is a mouthful people often use the term SP even when they are referring to the entire excitation itself and therefore they technically mean SPP.

Also, it is worth to be mentioned that throughout the paper we use $s a_{\mathrm{b}}{ }^{0}$ annotation to denote bounding mode propagating along the $\mathrm{Au} / \mathrm{Si}$ interface. The two letters in this mode annotation represent the symmetry or asymmetry of the fields in the vertical and horizontal axes respectively, while the subscript notes the bound nature of the mode, and the superscript indicates the mode order (also number of times the fields change sign in the horizontal direction in the metal). There are two visualization along with the Fig. 1 in the chapter 3 of this thesis which show a nice video of the discussed asymmetric SPP mode. 


\section{Photonics for Energy}

PhotonicsforEnergy.SPIEDigitalLibrary.org

\section{Surface plasmon enhanced photodetectors based on internal photoemission}

Mohammad Alavirad

Langis Roy

Pierre Berini 


\subsection{Introduction}

Surface plasmon polaritons (SPPs) are plasma oscillations of free electrons coupled with optical fields localized along the interface of a metal and a dielectric [33, 34]. SPPs are promising for several applications including optical data centre interconnects $[25,35,36,37]$, solar cells $[38$, $39,40]$ and bio(chemical) sensors $[41,42,43,44,45,46]$. In such applications surface plasmons (SPs) are excited by light and supported on a variety of metal and dielectric structures including planar arrangements of metal and dielectric films [47], metal gratings (or hole arrays) [36, 48, 49], a prism [50], a metal edge [51, 52], and metal nanoparticles such as islands, spheres and rods [53, 54], or nanoantennas $[55,56]$. The ability of SPs to resonate or propagate on metallic structures enables them to have interesting and useful attributes such as high bulk and surface sensitivities, sub-wavelength confinement and energy asymptotes in dispersion curves which have led to many different applications.

Recently the decay of plasmons into hot carriers (electrons or holes) has attracted considerable interest [57] due to applications in energy harvesting, photocatalysis and photodetection [58]. Specifically, an SP photodetector is a device capable of converting an optical signal to an electrical signal by detecting surface plasmons (light) or involving them in the photodetection mechanism. Such photodetectors typically combine a metallic structure that supports SPPs with photonic detection based on internal photoemission (IPE) or electron-hole pair (EHP) creation. IPE is useful for detection of light at energies below the bandgap of the semiconductor when incident radiation of energy $h v$ is greater than the Schottky barrier energy, $\Phi_{\mathrm{B}}$, and is of particular interest to extend the detection bandwidth of technologically important materials such as Si [59]. However, the creation of EHPs in the semiconductor is more efficient and dominates over IPE when $h v$ is greater than the bandgap energy of the semiconductor $E_{\mathrm{g}}$. In terms of photodetection, hot (energetic) 
carrier devices are typically formed by placing the metal surface in contact with a semiconductor $[35,60]$, forming a Schottky barrier. Hot carriers generated from the nonradiative decay of SPPs can transport to the metal-semiconductor interface and be injected into the conduction band of the semiconductor, resulting in photocurrent [61]. The main advantages of these photodetectors reside in their extremely high speed, in their simple fabrication process and small dimensions, comparable to those of highly integrated electronic circuits $[54,56]$.

The internal quantum efficiency of IPE photodetectors is low compared to that of photodetectors based on EHP, and this limits their application in power monitoring and in the telecommunications fields. The low quantum efficiency is a direct result of hot carrier transport limitations, initially through the metal in which they are generated (due to scattering), then across the Schottky barrier and into the semiconductor, where hot carriers which do not have enough energy to overcome the Schottky barrier are reflected and do not contribute to photoemission. An additional factor limiting the external quantum efficiency (responsivity) is the low absorption of incident light by metals, as they are highly reflective.

In recent years, new approaches and structures have been proposed in order to overcome such limitations. The generation of hot carriers in plasmonic nanostructures is a very promising energy conversion mechanism, and it has found interesting applications in photovoltaic and photocatalytic devices. Clavero has reviewed the fundamentals of hot-electron generation, injection and regeneration in plasmonic nanostructures allowing optimum utilization of the solar spectrum [57], highlighting interesting applications in photovoltaic and photocatalytic devices. This new energyconversion method holds the promises of high conversion efficiencies, while keeping fabrication costs low. Progress on SP photodetectors has been rapid, and interest on the topic continues to increase, prompting a review of most recent works in this area. Brongersma et al. [62] and Berini 
[63] highlight many opportunities for the generation and use of plasmon-induced hot carriers in nanostructures. Our objectives in this review are to describe the operating principles behind SP photodetectors based on IPE, summarize the most recent progress in this area, and highlight avenues that appear promising.

\subsection{Operating Principles of IPE-based Devices}

We first begin with some theoretical background useful to clarify the working principles of IPEbased devices. An $e^{+j \omega t}$ time-harmonic dependence is assumed throughout. The relative permittivity is denoted $\varepsilon_{\mathrm{r}}$, and is written for a metal in terms of real and imaginary parts as $\varepsilon_{\mathrm{r}, \mathrm{m}}=$ $-\varepsilon_{\mathrm{R}}-j \varepsilon_{\mathrm{I}} . \boldsymbol{k}$ denotes a generic wavevector and $k$ its magnitude. $k_{0}=2 \pi / \lambda_{0}=\omega / c_{0}$ is the wavenumber of plane waves in vacuum, $\lambda_{0}$ the wavelength in vacuum, $c_{0}$ the speed of light in vacuum and $\omega=$ $2 \pi v$ is the angular frequency.

\subsubsection{Single Interface SPPs}

Fig. 1 shows the simplest planar structure sustaining SPPs is that of a single, flat interface between a dielectric, non-absorbing half space $(y>0)$ with positive real dielectric constant $\varepsilon_{\mathrm{r}, \mathrm{d}}$ and an adjacent conducting half space $(y<0)$ described via a dielectric function $\varepsilon_{\mathrm{r}, \mathrm{m}}(\omega)$. This structure is termed the single interface SPP waveguide. The metallic character requires that $\operatorname{Re}\left\{\varepsilon_{\mathrm{r}, \mathrm{m}}(\omega)\right\}<$ $0\left(\varepsilon_{\mathrm{R}}>0\right)$. For metals this condition is fulfilled at frequencies below the bulk plasmon frequency $\omega_{\mathrm{p}}[33]$. 


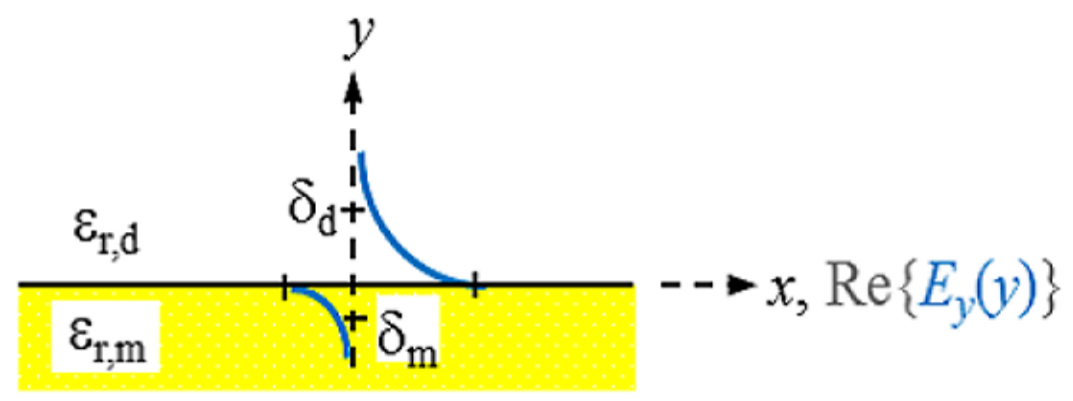

Fig. 1 (Adapted from [64]) Single-interface SPP and waveguide. The relative permittivity of the metal is denoted $\varepsilon_{\mathrm{r}, \mathrm{m}}$ and that of the dielectric $\varepsilon_{\mathrm{r}, \mathrm{d}}$. The distribution of the main transverse electric field component $\left(E_{\mathrm{y}}\right)$ of the SPP is sketched in blue on the structure.

Highly conductive metals and good dielectrics are usually used to implement SPP structures. $\mathrm{Au}$ is a relevant and good choice given its chemical stability and low loss among the other metals. $\mathrm{Ag}$ and $\mathrm{Cu}$ are also good choices but they are reactive so passivation is required. Other good choices are metals satisfying the condition $\varepsilon_{\mathrm{R}}>\varepsilon_{\mathrm{I}}$; nevertheless, many other materials may offer advantages for collecting these hot carriers. In [65] by considering the expected hot carrier distributions based on the electron density of states (EDOS) for 16 different materials ranging from metals (Au, Ag, Cu, Al, Fe, Pt, Ti, and Y), alloys (Ag-Cu, Al-Ga, Au-Pt, and AlOCu), and nanostructures (Ag and $\mathrm{Au}$ nanowires, carbon nanotubes, and graphene), the authors predict the preferred hot carrier type for collection and their expected performance under different illumination conditions. Proper material selection is then achieved by knowing carrier energy distributions, which are extremely sensitive to the details of the electronic bandstructure. For example $\mathrm{Cu}$ and $\mathrm{Au}$ generate hot holes that are much more energetic than electrons, while Ag and Al distribute energies more equitably between electrons and holes [66].

Metals are dispersive at optical wavelengths. Away from intraband transitions, the permittivity can be accurately modeled via the Drude equation which captures their dispersive character [33]:

$$
\varepsilon_{r, m}=-\varepsilon_{R}-j \varepsilon_{I}=1-\frac{\omega_{p}^{2}}{\omega^{2}+1 / \tau^{2}}-j \frac{\omega_{p}^{2} / \tau^{2}}{\omega\left(\omega^{2}+1 / \tau^{2}\right)}
$$


where $\omega_{\mathrm{p}}$ is the plasma frequency and $\tau$ the relaxation time. The Drude region of the permittivity of a real metal corresponds to the region of the electromagnetic spectrum where Eq. (1) is approximately satisfied, which for several metals runs from the visible to the infra-red. The permittivity approaches that of a perfect electric conductor as the wavelength increases into the far-infrared and beyond.

The single-interface SPP waveguide supports non-radiative SPP mode (one-bound) that is transverse magnetic (TM) and may propagate at any angle in the plane of the interface. The SPP fields $\left(E_{\mathrm{y}}, E_{\mathrm{z}}\right.$ and $\left.H_{\mathrm{x}}\right)$ are confined in the $y$ direction, with maxima at the interface, and decaying into both media. The main transverse electric field component $\left(E_{\mathrm{y}}\right)$ of the SPP is sketched on the structure of Fig. 1 as the thick curve. The field penetration in the metal $\delta_{\mathrm{m}}$ is much smaller than that in the dielectric $\delta_{\mathrm{d}}$. The SPP is confined because the metal and dielectric have $\operatorname{Re}\left\{\varepsilon_{\mathrm{r}}\right\}$ of opposite signs at the wavelength of interest. The wavenumber of the single-interface SPP is given by [33]:

$$
k_{S P P}=k_{0}\left(\frac{\varepsilon_{r, m} \varepsilon_{r, d}}{\varepsilon_{r, m}+\varepsilon_{r, d}}\right)^{1 / 2}
$$

For a dielectric cladding that has no loss $\left(\operatorname{Im}\left\{\varepsilon_{r, d}\right\}=0\right)$ the above simplifies to the following approximate expressions for the real and imaginary parts of the wavenumber [33]:

$$
k^{\prime} \cong k_{0}\left(\frac{\varepsilon_{R} \varepsilon_{r, d}}{\varepsilon_{R}-\varepsilon_{r, d}}\right)^{1 / 2} \text { and } k^{\prime \prime} \cong k_{0} \frac{\varepsilon_{I}}{2 \varepsilon_{R}^{2}}\left(\frac{\varepsilon_{R} \varepsilon_{r, d}}{\varepsilon_{R}-\varepsilon_{r, d}}\right)^{3 / 2}
$$

Fig. 2 gives the dispersion of the SPP ( $E=h v$ in eV versus $k^{\prime}, h$ is Planck's constant) on a $\mathrm{Ag} / \mathrm{SiO}_{2}$ single-interface [64] with the light-line in $\mathrm{SiO}_{2}$ plotted as the dash-dot curve. As the frequency decreases, the metal approaches a perfect electrical conductor, so the SPP confinement decreases and its dispersion merges with the light-line. As the frequency increases, the SPP dispersion approaches the energy asymptote, apparent in Fig. 2 near $E \sim 3.4 \mathrm{eV}\left(\lambda_{0} \sim 360 \mathrm{~nm}\right)$, and $k^{\prime}$ becomes large. 


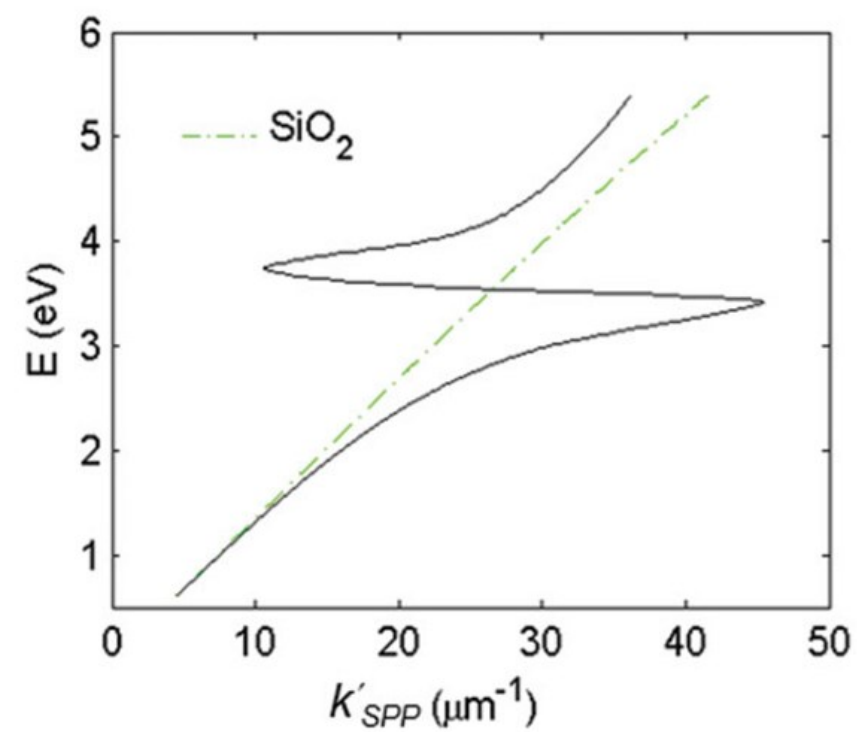

Fig. 2 (Adapted from [64]) Dispersion curve of the SPP along a $\mathrm{Ag} / \mathrm{SiO}_{2}$ semi-infinite interface; the light line in $\mathrm{SiO}_{2}$ is plotted as the green dash-dot curve.

The optical density-of-states increases and the group velocity decreases near the energy asymptote. Bend-back in the SPP dispersion is observed for frequencies above the asymptote $(h v$ $>3.4 \mathrm{eV}$ ), linking the bound SPP on the right of the light-line to the radiative SPP on the left. The bound SPP (right of the light line - Fig. 2) cannot be directly excited by incident light - an additional structure is needed in order to increase the in-plane wavenumber of the light to match that of the SPP; a corrugated grating or a prism is commonly used to accomplish this task [33].

\subsubsection{Internal photoemission (IPE)}

A conventional Schottky photodiode on n-type Si is sketched in Fig. 3(a) [67], and used to describe two detection mechanisms that can be used to detect SPPs. The structure is convenient for the detection of SPPs because the latter may propagate along its metal surfaces, particularly along the metal-Si interface. 

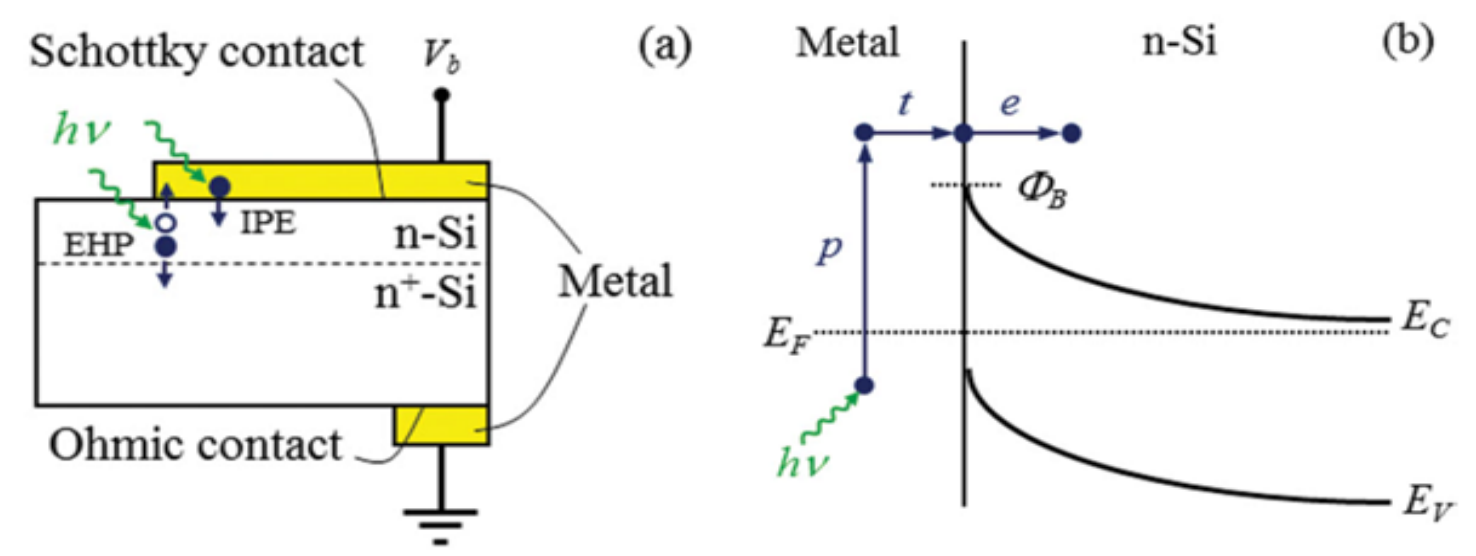

Fig. 3 (Adapted from [63]) (a) Schottky diode on n-Si illuminated by light of photon energy $h v$ (green). Reverse biasing $\left(V_{\mathrm{b}}<0\right)$ is assumed. Electrons are shown by filled dark blue circles and holes by unfilled ones. (b) Energy band diagram of a Schottky contact on n-Si and the 3-step internal photoemission process consisting of $p-$ photoexcitation, $t$-transport, $e-$ emission. $E_{\mathrm{C}}$ and $E_{\mathrm{V}}$ are the conduction and valence band edges, respectively, $E_{\mathrm{F}}$ is the Fermi level, and $\Phi_{\mathrm{B}}$ is the Schottky barrier height.

Two mechanisms for photodetection are sketched in Fig. 3(a): The first is creation of electronhole pairs (EHPs) in Si due to the absorption of incident radiation of energy $h v$ greater than the bandgap energy $E_{\mathrm{g}}$. This mechanism, labeled EHP, involves the 3 steps of optical absorption and creation of EHPs, separation of EHPs, and transport under reverse bias for collection as the photocurrent. The second is the internal photoemission (IPE) of hot carriers (electrons in this case) created in the metal due to absorption of incident radiation of energy $h v$. This mechanism, labeled IPE, is described via the energy band diagram of Fig. 3(b): IPE is also a 3-step process consisting of photoexcitation of hot (energetic) carriers in the metal, transport with scattering of hot carriers towards the metal-Si interface, and the emission of hot carriers over the Schottky barrier into the semiconductor where they are collected as the photocurrent. IPE requires that $h v$ be greater than the Schottky barrier energy $\Phi_{\mathrm{B}}$, and is a useful process for $\Phi_{B}<h v<E_{\mathrm{g}}$, i.e., for detection at energies below the semiconductor bandgap. 
The internal quantum efficiency $\eta_{\mathrm{i}}$ is defined as the number of carriers that contribute to the photocurrent $I_{\mathrm{p}}$ per absorbed photon per second:

$$
\eta_{i}=\frac{I_{P} / q}{S_{a b s} / h v}
$$

where $S_{\text {abs }}$ is the absorbed optical power and $q$ is the electronic charge.

For detection using IPE, if $h v<<E_{\mathrm{F}}$ and there is no bandgap then it can be assumed that the density of states is approximately constant, so $\eta_{\mathrm{i}}$ is given approximately by [68]:

$$
\eta_{i}=\frac{1}{2}\left(1-\sqrt{\frac{\Phi_{B}}{h v}}\right)^{2}
$$

for absorption in the metal only and near the Schottky contact. Example Schottky barriers are $\Phi_{\mathrm{B}}$ $=0.34,0.8,0.58$ and $0.72 \mathrm{eV}$ for $\mathrm{Au} / \mathrm{p}-\mathrm{Si}, \mathrm{Au} / \mathrm{n}-\mathrm{Si}, \mathrm{Al} / \mathrm{p}-\mathrm{Si}$ and $\mathrm{Al} / \mathrm{n}-\mathrm{Si}[67]$. At $\lambda_{0}=1310 \mathrm{~nm}, \eta_{i}$ ranges approximately from $0.3 \%$ to $9 \%$. The external quantum efficiency $\eta_{\mathrm{e}}$ and responsivity $R$ describe how a detector performs upon insertion in a system. $\eta_{\mathrm{e}}$ is defined as:

$$
\eta_{e}=\frac{I_{P} / q}{S_{\text {inc }} / h v}
$$

where $S_{\text {inc }}$ is the incident optical power. $\eta_{\mathrm{e}}$ and $\eta_{\mathrm{i}}$ are related by:

$$
\eta_{e}=A \eta_{i}
$$

where $A$ is the absorptance, given by:

$$
A=\frac{S_{a b s}}{S_{i n c}}
$$

The responsivity of a photodetector is given by the ratio of the photocurrent to the incident optical power and can be expressed in terms of $\eta_{\mathrm{i}}$ and $\eta_{\mathrm{e}}$ as:

$$
R=\frac{I_{p}}{S_{\text {inc }}}=\frac{\eta_{e} q}{h v}=\frac{A \eta_{i} q}{h v}
$$

IPE can be enhanced in several plasmonic structures as discussed in the following sections. It should be mentioned that the creation of EHPs can also be enhanced by involving SPPs. Also it is 
important to mention that plasmon hot carrier generation results in higher-energy electrons than direct excitation [69].

The probability for photon absorption is proportional to the square of the local electric field inside the metal. Planar metal surfaces reflect most of the incident light, and light absorption is not very efficient. The importance of plasmons to enhance light absorption in the near-surface region of a metal and consequently enhance photoemission processes has been highlighted in the past [70]. In metallic nanostructures light absorption can be further enhanced by exciting localized surface plasmon resonances. This produces an antenna effect resulting in light collection from an area that is larger than the physical size of the nanostructure [71]. The associated light concentration has been verified by, and used in, surface-enhanced processes, including Raman spectroscopy [72], photochemistry [73], and the photoexcitation of nearby quantum emitters [56]. Also knowledge of the imaginary part of the permittivity of a metal provides a means for optimizing hot carrier generation. For instance, by tuning a plasmon mode to energies where the imaginary part of the permittivity is large, maximizing the hot carrier production is possible. This tuning can be performed either by exploiting the unique geometrical tunability of localized surface plasmon resonances (LSPRs) of metallic nanoparticles, or by fabricating composite structures consisting of both noble metals [74] and metals with strong intraband transitions (such as transition metals) [75].

Fig. 4(a) shows photoexcitation following the illumination of a metal nanoparticle with a femtosecond source pulse. Excitation of SPPs guides the flow of light (Poynting vector) towards and into the nanoparticle. Larger nanoparticles and shorter lifetimes result in higher carrier production rates but smaller energies [76]. By following the trajectories of photoemitted electrons created by intense laser pulses, researchers have also been able to map the evanescent fields around 
plasmonic nanoparticles. In Fig. 4(b) one can see that electrons are preferentially emitted from regions of high field intensity (hot spots) and subsequently accelerated within the evanescent tail of plasmons resonating on nanoparticles.

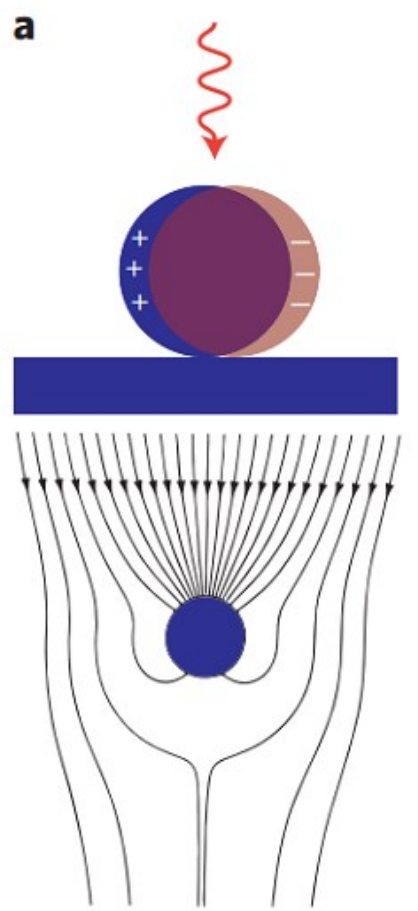

b

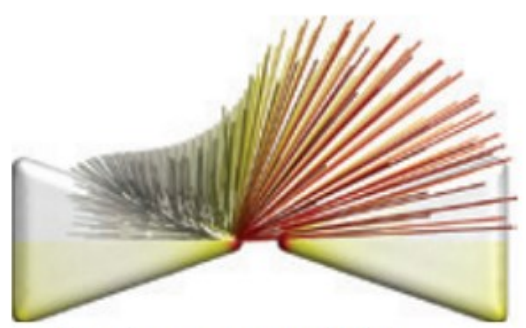

Electron energy (eV)

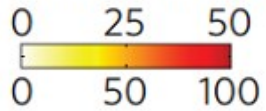

Fig. 4 (a) (Adapted from $\left[62,{ }^{77}\right]$ ) Photoexcitation processes following the illumination of a metal nanoparticle with a femtosecond laser pulse; the excitation of a localized surface plasmon redirects the flow of light (Poynting vector) towards and into the nanoparticle, (b) (Adapted from [78]) Simulation of field enhancement in a plasmonic bowtie nanoantenna and the resulting electron trajectories.

This enables the steering of photoemitted carriers in directions determined by particle geometry [78]. Precisely designed plasmonic nanostructures can significantly enhance photon absorption and convert incident light into hot carriers [79].

\subsection{IPE-based Photodetectors}

A Schottky barrier photodetector formed at the interface between a metal and a lightly doped semiconductor can be used to detect infrared radiation below the bandgap energy of the substrate via IPE $[2,80]$. Such detectors involving SPP excitations in prism [81], grating-coupled detectors 
[5], waveguides [35, 82] and nanoparticles and nanoantennas $[12,41,60,83,84]$ have been reported; prism coupling to SPPs on a suitable detection structure was one of the first types of SPP detectors investigated in [85]. The Otto coupling configuration used in these studies has been more popular in experimental work, mostly because the detection structures can be fabricated independently, then the prism aligned to the detector in the setup [63].

\subsubsection{Grating Detectors}

A grating structure can be used as an alternative to a prism to increase the in-plane momentum of the incident light in order to match that of the SPP [33] providing compactness and manufacturing advantages and high detection sensitivity. In particular, the grating coupling method is convenient to excite SPPs on a detector $[48,51]$, because the arbitrary wavenumber of the diffracted light can be generated by simply changing the grating pitch. The coupling condition of an SPP at a metal surface containing a grating is described by the following equation:

$$
k_{s p p}=k_{0} \sin \left(\theta_{i}\right)+M \frac{2 \pi}{\Lambda}
$$

where $k_{s p p}$ is the wavenumber of the SPP parallel to the metal surface, $k_{0}$ is the wavenumber of the incident light, $\theta_{i}$ is the angle of incidence of the light, $M$ is the grating order and $\Lambda$ is the grating pitch. Gold nanogratings on Si have been shown to successfully couple incoming optical light to SPPs excited at the nanograting-Si interface [86]. A metal-semiconductor (Schottky) nanograting structure has been proposed as an SPP photodetector [87, 88], sensitive to the angle of incidence, polarization and wavelength of light. The SPP photodetector consists of a Au/Si Schottky diode with a grating also used as the metal contact, and the detector converts SPP signals into photocurrent [89]. Because the responsivity of the SPP detector is closely related to the SPP intensity at the $\mathrm{Au} / \mathrm{Si}$ interface, the grating structure can be optimized in terms of the slit pitch and film thickness $[24,25]$. 
Fig. 5 shows a novel grating structure forming an Au/Si Schottky type SPP detector, producing a responsivity of $38 \mu \mathrm{A} / \mathrm{W}$ (under no electrical bias) [24].
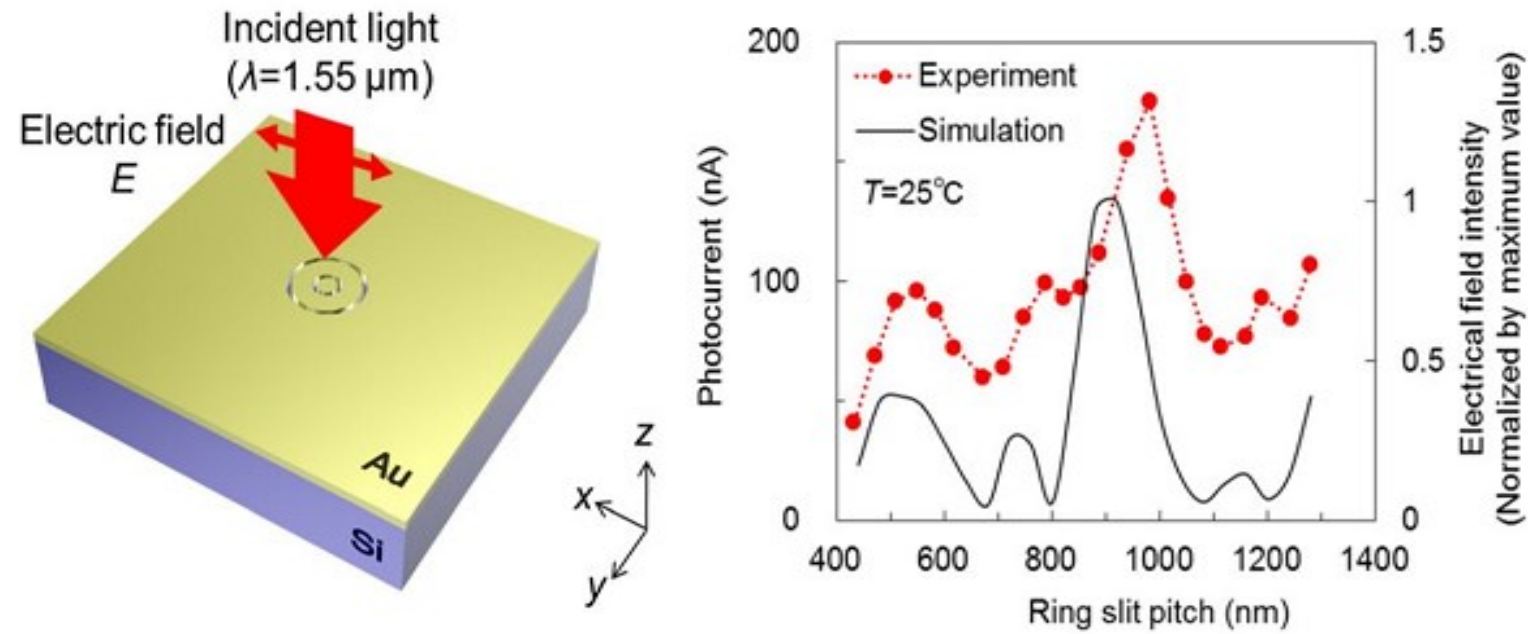

Fig. 5 (Adapted from [24]) (left) Structure of Au/Si Schottky-type SPP detector with a nanoslit grating. (right) Slit pitch dependencies on SPP intensity and photocurrent for ring type grating when slit pitch varies in the range of 440 $\mathrm{nm}$ to $1280 \mathrm{~nm}$

An efficient nano-slit grating arrangement that affects the phase conditions at both the air/Au interface and the $\mathrm{Au} / \mathrm{Si}$ interface has been studied. When the slit pitch was nearly equal to an integral multiple of the SPP wavelength at the Au/Si interface, a higher intensity SPP mode was excited at the $\mathrm{Au} / \mathrm{Si}$ interface outside the slits. Also, while the phase matching conditions were satisfied at the $\mathrm{Au} / \mathrm{Si}$ interface, the anti-phase interference of the SPP mode along the air/Au interface outside the slits was also confirmed. Experimentally, similar results were confirmed as an SPP photodetector with a ring-type grating that has a polarization-independent photocurrent.

Light coupling, guiding and photodetection can be combined in the same structure. Fig. 6 shows a simple metal/semiconductor SPP structure consisting of a SPP waveguide and a detector where the SPP couples from the waveguide to the photodetector [25]. The air/Au interface is used for guiding SPPs and the SPPs propagate at this interface without large losses. To convert the SPPs into an electrical signal using internal photoemission, the propagating SPPs are coupled to the 
$\mathrm{Au} / \mathrm{Si}$ interface by the diffraction structure consisting of a multi-slit structure and a disk array. A tunable laser was set to the wavelength of $1550 \mathrm{~nm}$, emitting an optical power in the range of 0 to $10 \mathrm{~mW}$. The photocurrents detected at the $\mathrm{Au} / \mathrm{Si}$ interface are much larger when compared with those detected for the device without the diffraction structure (26 times for the multi-slit structure and 10 times for the disk array). This kind of device is targeted to be used as a low-loss SPP waveguide and detector in photonic integrated circuit applications and wavelength filters controlled by the slit pitches.

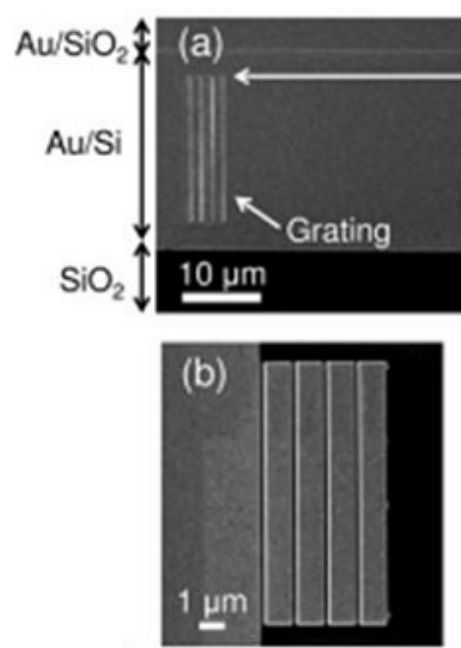

(e)

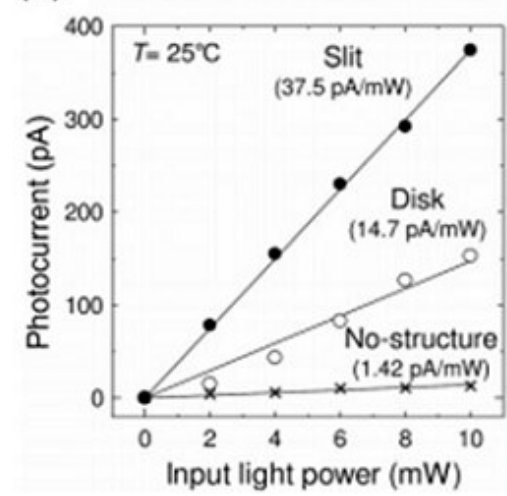

\section{$100 \mu \mathrm{m}$}
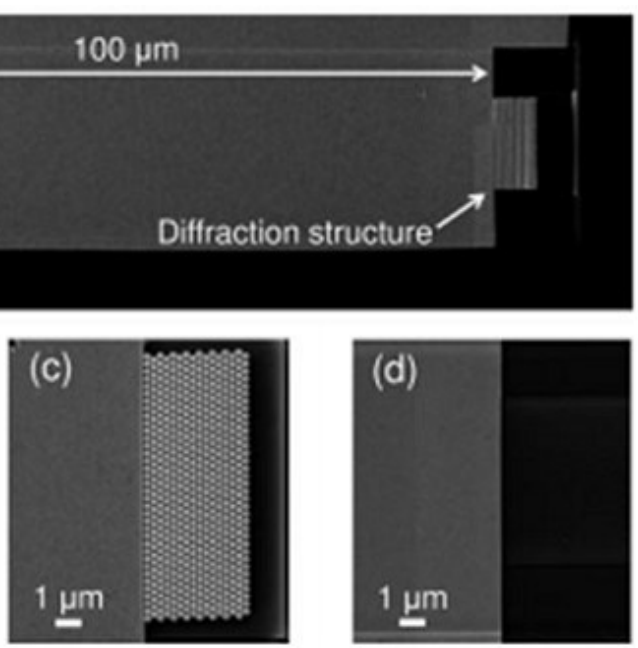

(f)

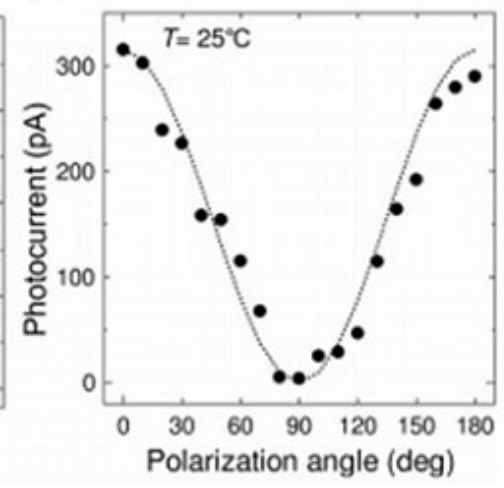

Fig. 6 (Adapted from [25]) Scanning electron micrographs (SEMs) of (a) a device used to evaluate the photoresponse measured by the devices with diffraction structures, (b) the multi-slit structure, (c) the disk array structure, and (d) no diffraction structure in place, (e) Photoresponses of the devices with each of the diffraction structures, (f) Polarization angle dependence of the photocurrent detected by the device with the multi-slit structure. 
Sobhani et al. presented a grating Schottky photodetector based on IPE with the geometry shown in Fig. 7, enabling three times narrower spectral response than observed for nanoantenna-based devices [58]. The responsivity of this device $(\sim 0.6 \mathrm{~mA} / \mathrm{W}$ at zero bias voltage $)$ is similar to responsivities reported in the literature for SPP detectors [35].

The internal quantum efficiency of this photodetector is $\sim 0.2 \%, 20$ times larger than the previously reported literature value of $0.01 \%$ for nanoantenna-based devices [60]. Unlike previous devices, this responsivity maintains its narrow full width at half maximum (FWHM) bandwidth of nominally $54 \mathrm{meV}$, narrower by a factor of more than three compared to previously reported devices [60].
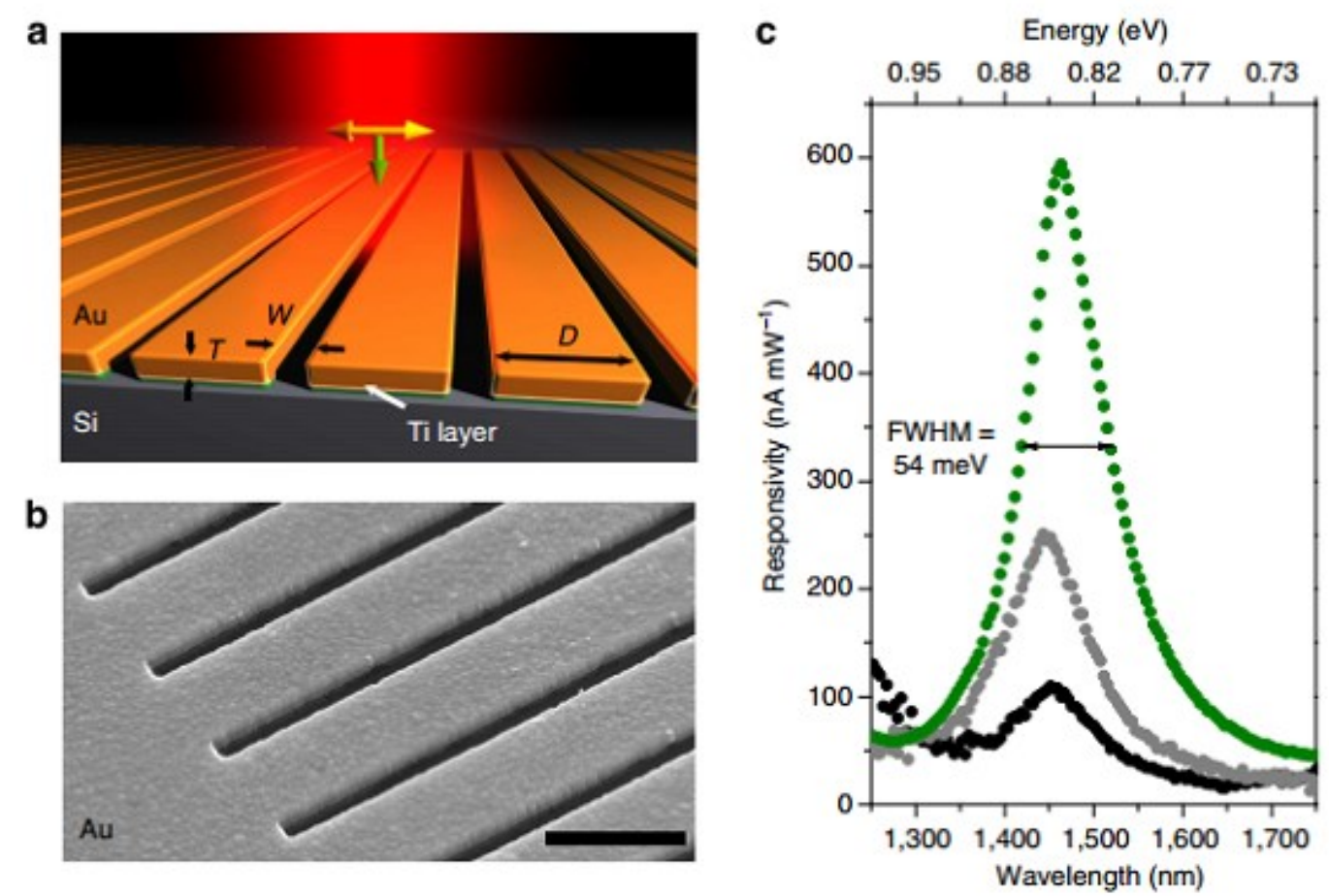

Fig. 7 (Adapted from [58] (a) Schematic of a gold grating on an n-type Si with a 2-nm Ti adhesion layer, oriented transverse to the laser polarization. The polarization of the incident beam and its $k$ vector are represented by yellow and green arrows, respectively, (b) SEM image of a gold grating structure with a grating thickness $(T)=200 \mathrm{~nm}$, interslit distance $(D)=950 \mathrm{~nm}$ and slit width $(W)=250 \mathrm{~nm}$. For all structures the array measured $12 \times 12 \mu \mathrm{m}^{2}$. The scale bar is $1 \mu \mathrm{m}$. (c) Responsivities of grating-based photodetectors for three different gold layer thicknesses, $T=$ $93 \mathrm{~nm}$ (black), $170 \mathrm{~nm}$ (grey) and $200 \mathrm{~nm}$ (green), showing a strong intensity dependence on grating thickness. 
Although studies have been carried out on various aspects of nanogratings and their detection applications, achieving a high infrared (sub-bandgap) responsivity over a broad spectral range on low-cost Si remains a difficult (and worthwhile) challenge. In [90] Alavirad et al. proposed a novel plasmonic Schottky photodetector consisting of a Au nanograting on a thin metal patch on $p$-type $\mathrm{Si}$, as shown in Fig. 8, providing detection via the IPE of hot holes generated in the patch along the Schottky contact. This structure produced some of the highest responsivities reported to date in the literature, of about $13 \mathrm{~mA} / \mathrm{W}$ (with no electrical bias) at $\lambda_{0} \sim 1550 \mathrm{~nm}$. The RC time constant limited electrical bandwidth of this device is predicted to be up to $\sim 26 \mathrm{GHz}$. Gathered into arrays, these small area devices are capable of aggregate bandwidths into the $\mathrm{Tb} / \mathrm{s}$ range. These detectors have many important advantages such as simplicity, compatibility with silicon, and low-cost fabrication.
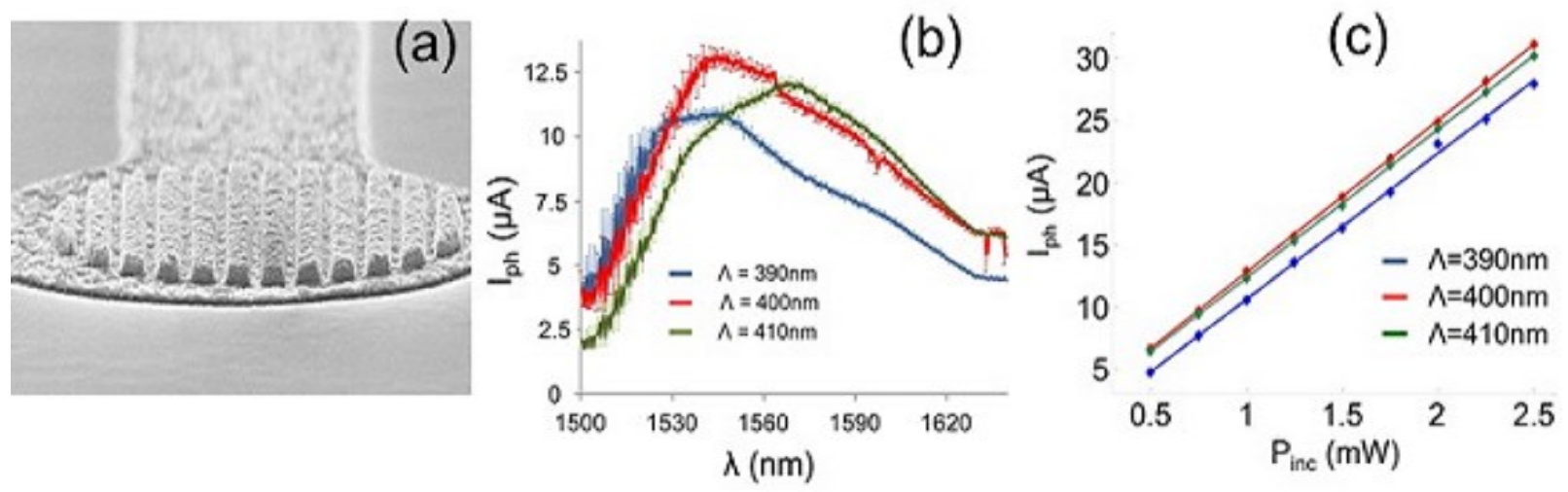

Fig. 8 (Adapted from [90]) (a) Scanning electron micrographs of an uncoated Au/p-Si nanograting photodetector. The pitch of the device is measured of $406 \mathrm{~nm}$ (400 nm designed) and the thickness of the Au patch is measured as $29.8 \mathrm{~nm}$ (20 nm designed) and its duty cycle of this is $60 \%$, (b) Measured photocurrent response of three grating photodetectors; $V_{\mathrm{B}}=-100 \mathrm{mV}, \Lambda=390,400$ and $410 \mathrm{~nm}$, duty cycle of $62 \%$ and patch diameter of $25 \mu \mathrm{m}$, (c) Photocurrent generated by grating detectors $v s$. incident power $P_{\text {inc }}$ measured for three different pitches. A linear fit is applied to the data and the slope corresponds to the responsivity of the device. 


\subsubsection{MIM, MOM, MSM and MIS Detectors}

Other nanostructures investigated as SPP photodetectors include gratings or arrays of metal nanowires on metal-oxide-semiconductor structures (MOS) [30], metal-oxide-metal (MOM) structures [91], or on a commercial photodiode [92]. In these planar junctions, the excitation of propagating SPPs can enhance efficiency. Brongersma et al. have examined the phenomenon of photocurrent generation by hot electrons in wavelength-scale metal-insulator-metal (MIM) tunneling devices [93] and enhanced their efficiency by reshaping one of the metal contacts of the $\mathrm{Au} / \mathrm{Al}_{2} \mathrm{O}_{3} / \mathrm{Au}$ junction into an array of metal nanowires that support resonating SPPs, as depicted in Fig. 9. The voltage dependence, spectral dependence, nanowire width dependence and polarization dependence of the photocurrent were examined experimentally.

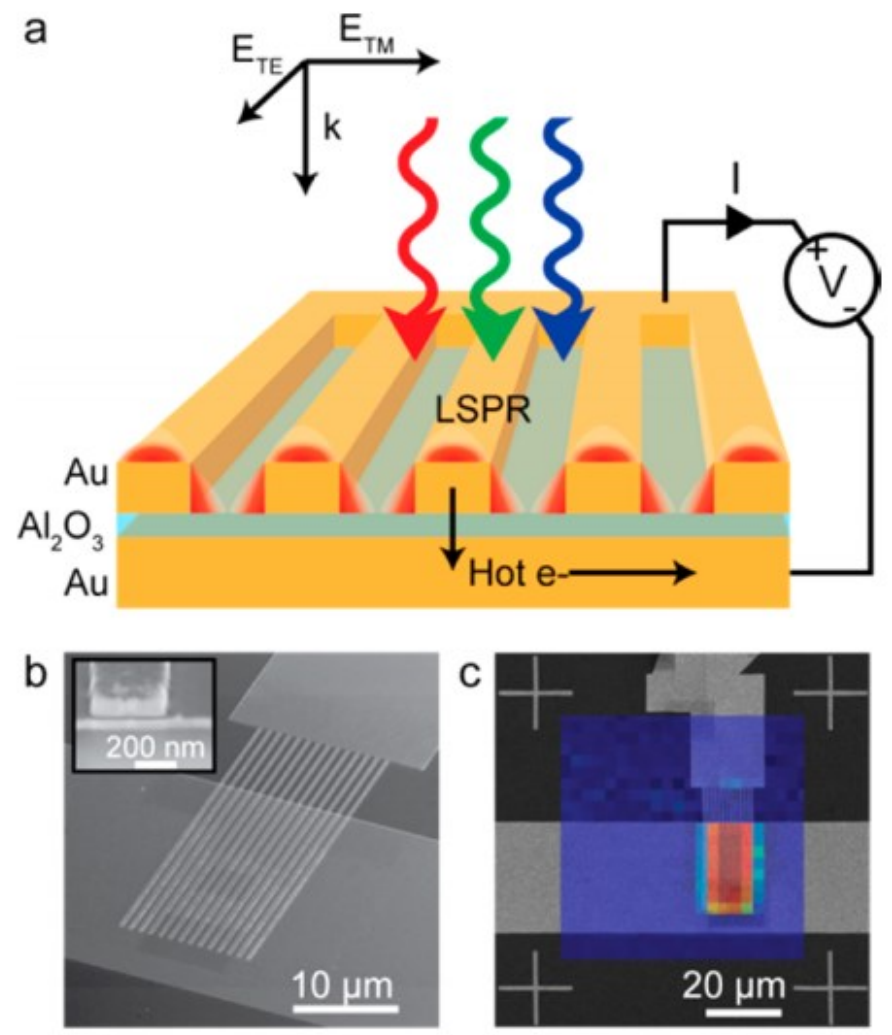

Fig. 9 (Adapted from [67]) (a) Schematic illustration of a typical device featuring a series of MIM crossbar junctions formed between a wide bottom electrode and a set of nanoscale top electrodes. The directions of the electric field for 
transverse electric (TE) and transverse magnetic (TM) illumination are as depicted. (b) SEM image of a device with a $470 \mathrm{~nm}$ wide top electrode. The inset shows a cross-sectional image of the device, composed of an oxide layer sandwiched by top and bottom metal layers. (c) Photocurrent image of the device taken at an illumination wavelength of $400 \mathrm{~nm}$ with TM polarization overlaid on the SEM image of the device. The red color corresponds to $7 \mathrm{pA}$ and blue indicates a close to zero photocurrent.

Fig. 9(c) shows an image photocurrent taken from a device with a $470 \mathrm{~nm}$ wide stripe overlaid on its SEM image (no bias voltage). It is clearly observed that photocurrent is generated only in the regions where the top electrodes overlay the wide bottom electrode to form a series of nanoscale MIM junctions. Finally, they measured the spectral dependence of the responsivity of a detector with $750 \mathrm{~nm}$ wide stripes upon illumination with TM polarized light with the bias voltages in the range from -0.4 to $+0.4 \mathrm{~V}$ and obtained a maximum value of $\sim 0.55 \mathrm{~mA} / \mathrm{W}$ in the visible range. In modern optical communication systems, high efficiency (realized from some parameters such as size, responsivity, dark current and photocurrent) photodetectors at the communication window with the wavelength of $1550 \mathrm{~nm}$ are strongly desired. Wu et al. designed an MIM IPEbased photodetector exhibiting an unbiased responsivity of $0.1 \mathrm{~mA} / \mathrm{W}$ and an ultra-narrow response band $(\mathrm{FWHM}=4.66 \mathrm{meV})$, which promises to be a candidate as the compact photodetector operating in communication band [94].

Casalino et al. presented a $\mathrm{Si}$ on insulator (SOI) waveguide Metal-Semiconductor-Metal (MSM) photodetector, based on IPE and working at a wavelength of $1550 \mathrm{~nm}$ [95]. Taking advantage of the MSM structure, their device is able to increase responsivity by lowering the barrier. At the same time, a very low dark current is achieved both by depositing a very small active metal area and by realizing a device with asymmetric electrodes [96]. Fig. 10(a) shows the SOI structure obtained by depositing the absorbing $\mathrm{Cu}$ metal strictly around the output vertical facet of a SOI rib waveguide. 

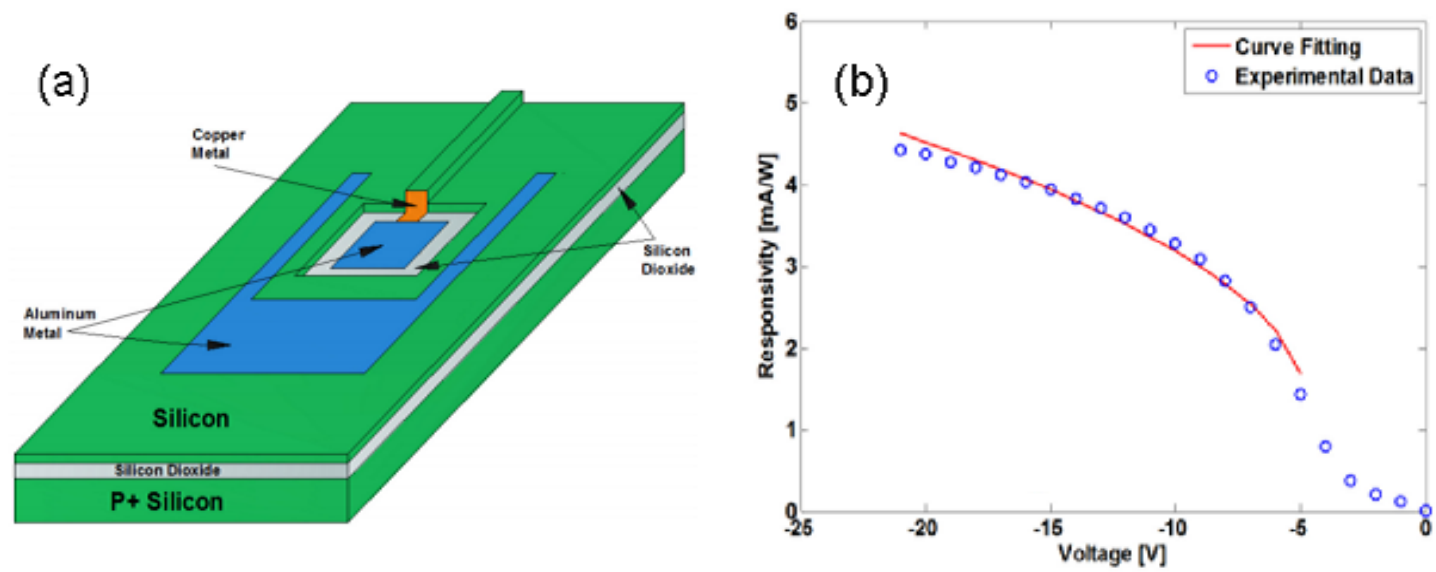

Fig. 10 (Adapted from [95]) (a) Schematic of the proposed photodetector integrated with SOI waveguide, (b)

Responsivity vs reverse voltage applied at $1550 \mathrm{~nm}$.

This active metal is in contact with a large Al pad deposited on the buried oxide layer of the SOI structure, and therefore does not contribute to the dark current or the junction capacitance. A second large Al electrode was deposited on the Si substrate to collect the generated photocurrent. In Fig. 10(b) the responsivity of MSM photodetector versus bias voltage measured at $1550 \mathrm{~nm}$ is shown. It reaches a maximum value of about $4.5 \mathrm{~mA} / \mathrm{W}$ at $-21 \mathrm{~V}$.

Another alternative for conventional metal-semiconductor Schottky junction is a choice of metal-insulator-semiconductor (MIS) heterojunction which reduces dark current significantly, resulting in increased device performance [97].

\subsubsection{Nanoparticles Detectors}

In recent years plasmonic energy conversion has been proposed as a promising alternative to conventional electron-hole separation in semiconductor structures. This emerging method is based on the generation of hot carriers in plasmonic devices through the decay of surface plasmons. LSPRs occur in well-designed nanostructures in which confined free electrons oscillate with the same frequency as the incident light and eventually enter resonance, giving rise to enhanced, highly localized electromagnetic fields. Therefore, such nanostructures have been investigated as very 
efficient light-trapping elements that can be integrated into photovoltaic cells to considerably increase the quantum efficiency of conventional schemes [57]. Many studies have considered plasmonic hot-electron generation with application to both photovoltaic and catalytic devices.

Many of these studies considered $\mathrm{Au}$ or $\mathrm{Ag}$ nanoparticles in contact with $\mathrm{TiO}_{2}[98,99,100$, 101], as shown in Fig. 11. The Au-Nanoislands (Au-NIs) annealed at $800{ }^{\circ} \mathrm{C}$ tightly contacted the $\mathrm{TiO}_{2}$ surface and appeared to be incorporated into the interfacial structure atomic layers. Electron energy loss spectroscopy (EELS) measurements were obtained to characterize the interfacial structure of the Au-NIs/TiO 2 . In EELS, the corresponding core edge (the titanium (Ti) $\mathrm{L}_{23}$ edge) in the spectrum provides information about the electronic nanostructure and symmetry coordination of the excited atom. Measurements show extinction spectra of $\mathrm{Au}-\mathrm{NIs} / \mathrm{TiO}_{2}$ prepared under $800{ }^{\circ} \mathrm{C}$ annealing. Although the extinction values at the peak wavelength are different, all the $\mathrm{Au}-\mathrm{NIs} / \mathrm{TiO}_{2}$ show plasmon peak at the wavelength range from $600 \mathrm{~nm}$ to $630 \mathrm{~nm}$.

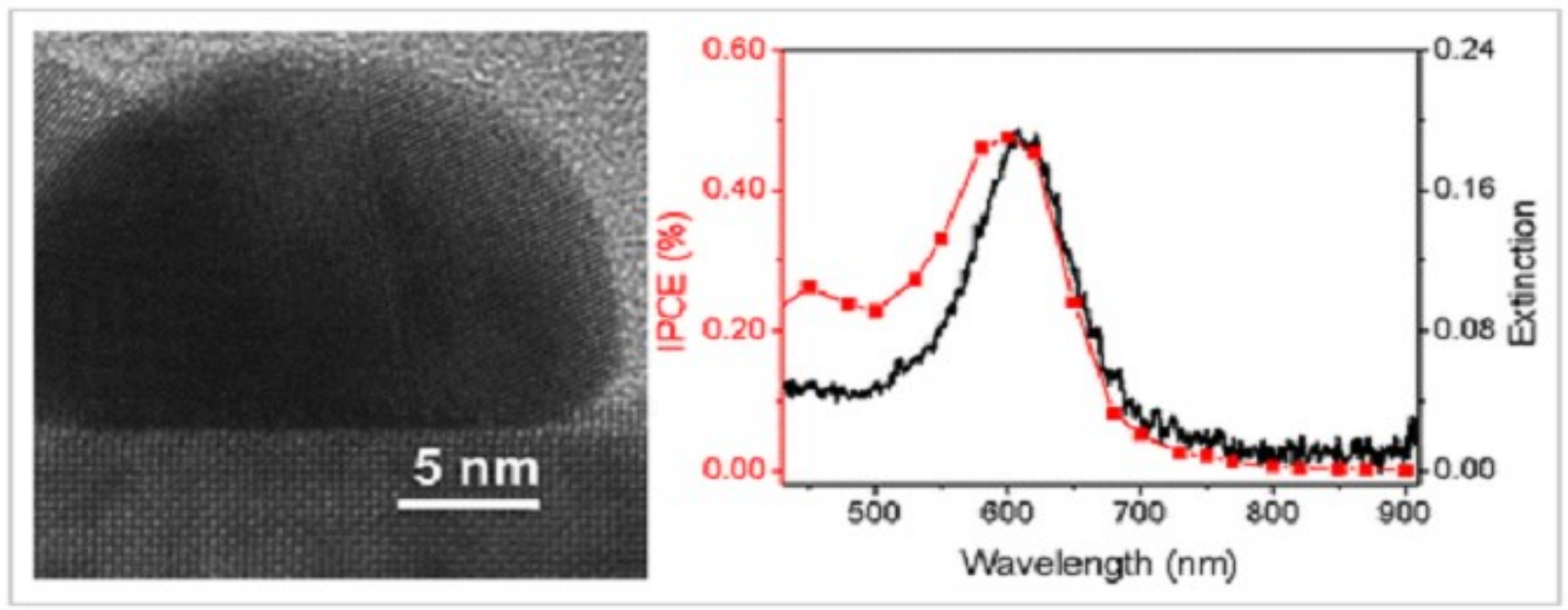

Fig. 11 (Adapted from [99]) (left) High-resolution transmission electron microscopy (HR-TEM) images of the cross-section at the interface of the $\mathrm{Au}-\mathrm{NIs}$ and the $\mathrm{TiO}_{2}$ annealed at $800^{\circ} \mathrm{C}$, (right) The incident photocurrent efficiency (IPCE) action spectrum measured with the $\mathrm{Au}-\mathrm{NIs} / \mathrm{TiO}_{2}$ photoelectrode (red) and the extinction spectrum of the Au-NIs/ $/ \mathrm{TiO}_{2}$ photoelectrode (black). The applied potential was $0.3 \mathrm{~V}$ versus a saturated calomel electrode 
In further recent developments, multiple combinations of materials have been proposed to enable hot carrier detectors and catalytic devices. Some examples include multilayer assemblies of $\mathrm{Pt}$ nanoparticles on $\mathrm{TiO}_{2}$ thin films [102], Ag-decorated $\mathrm{TiO}_{2}$ nanotube arrays [103] as shown in Fig. 12, ZnO nanorods decorated with Au nanoparticles [104], and hierarchical $\mathrm{Au}-\mathrm{ZnO}$ flowerrod heterostructures [105] as shown in Fig. 13.

Referring to Fig. 12, Au nanoparticles are well dispersed on the surface of $\mathrm{TiO}_{2}$ nanotubes of metallic state. The SPP resonance (SPR) effect of Ag nanoparticles extends the visible light response and consequently enhances the absorption capacity of $\mathrm{TiO}_{2}$. They can also restrain the recombination of photo-generated $\mathrm{EHPs}$ in $\mathrm{TiO}_{2}$ nanotubes efficiently. Experimental results prove that $\mathrm{Ag} / \mathrm{TiO}_{2}$ nanotube devices have better photocatalytic performance than pure $\mathrm{TiO}_{2}$ nanotubes and their corresponding hydrogen evolution rate is 3.3 times as that of the latter.

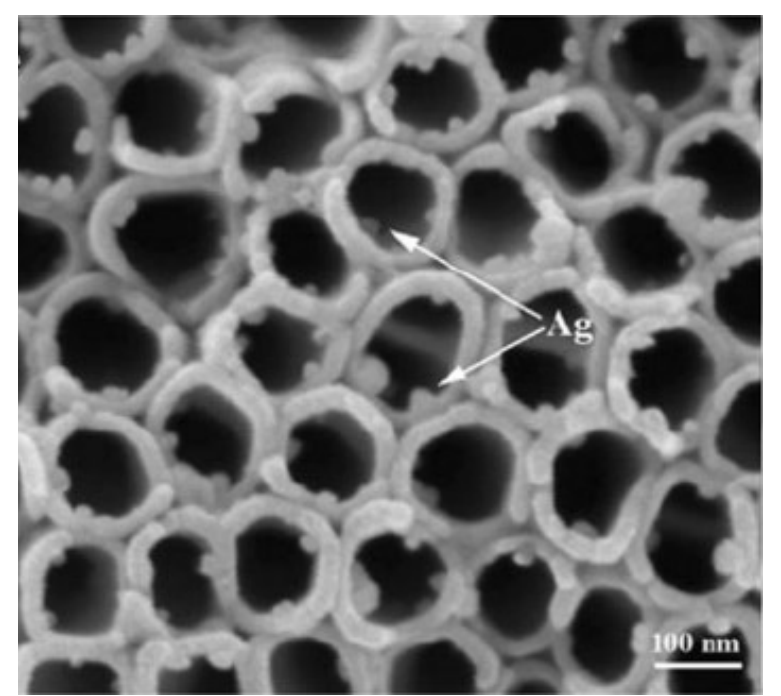

Fig. 12 (Adapted from [103]) SEM of Ag nanoparticles in $\mathrm{TiO}_{2}$ nanotubes

The $\mathrm{Au} / \mathrm{ZnO}$ samples in Fig. 13 are made through a thermal decomposition method. Au nanoparticles were loaded on oriented hierarchical $\mathrm{ZnO}$ flower-rod heterostructures. Measurements reveal that there is an electronic interaction between the Au nanoparticles and $\mathrm{ZnO}$ flower-rods, and more oxygen molecules are adsorbed on the surface of $\mathrm{Au} / \mathrm{ZnO}$ heterostructures. 
They show a strong absorbance in the visible region due to SPR and enhance the separation of EHPs, resulting in the improvement of photocatalytic activity under visible light radiation.

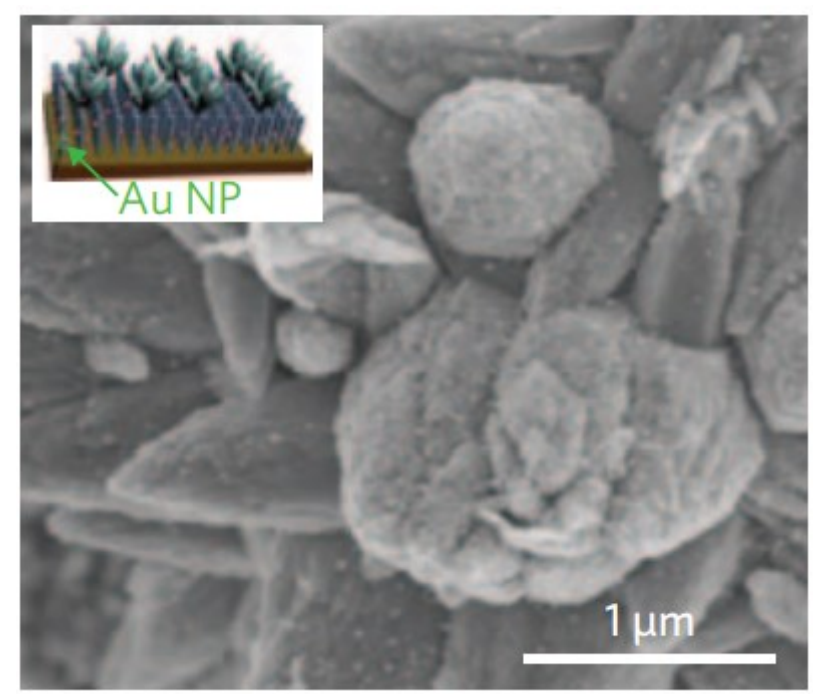

Fig. 13 (Adapted from [105]) SEM of Ag on $\mathrm{ZnO}$ flower-rod heterostructures.

Small metal nanoparticles exhibit resonant responses under optical excitation, dependent on the structure's shape, size and composition, the dielectric environment in which they find themselves, and the wavelength of illumination [54]. Nazirzadeh et al. presented a broadband near infra-red (NIR) Si Schottky photodetector consisting of Au nanoislands (randomly-sized) formed on an n-type Si substrate, fabricated by rapid thermal annealing of a thin Au film [12]. Their device structure is shown in Fig. 14. Its potential monolithic integration with electronics makes it promising for ultra-low-cost NIR imaging in large volume applications such as automotive and security, in addition to telecommunications. The measured photoresponse of the devices extend up to $\lambda_{0}=2 \mu \mathrm{m}$ and the responsivities at $1.3 \mu \mathrm{m}$ and $1.55 \mu \mathrm{m}$ are $2 \mathrm{~mA} / \mathrm{W}$ and $0.6 \mathrm{~mA} / \mathrm{W}$, respectively. 
(a)
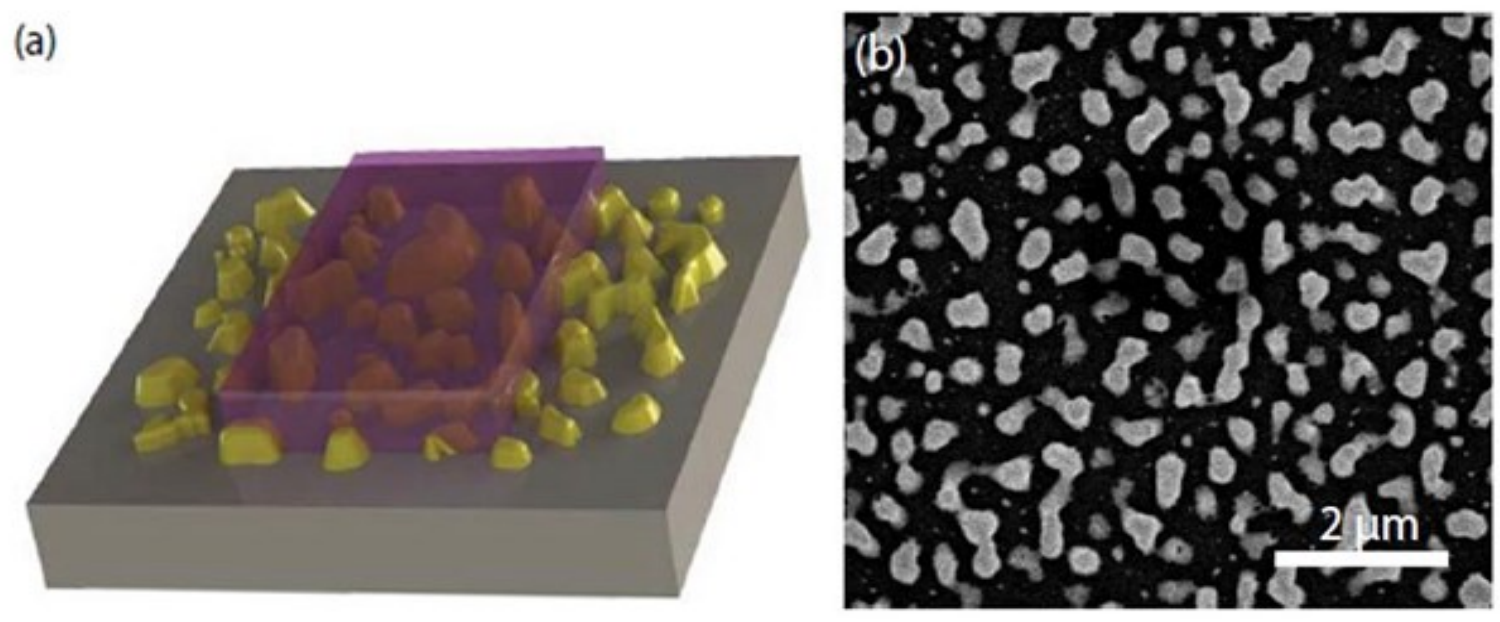

Fig. 14 (Adapted from [12]) (a) Depiction of broad-band NIR Si Schottky photodetector. Au nanoislands are formed on n-type Si by rapid thermal annealing of a thin Au film. Si substrate is used as the bottom contact; and $\mathrm{Au}$ nanoislands together with the AZO capping layer constitute the top contact, (b) SEM image of the samples annealed at $450^{\circ} \mathrm{C}$.

Several works have provided insight into the localization of hot electron generation in plasmonic nanostructures. Dombi et al. demonstrated the ultrafast generation of hot electrons from tailored metallic nanoparticles and realized the role of plasmonic field enhancement in this process by comparing resonant and off-resonant particles, as well as different nanorod geometries. These nanoparticles offer the possibility of tailoring nanoscale electric fields with high-level control over the plasmonic resonance, which can be overlapped with the spectrum of the exciting laser pulse to achieve field enhancements of the incident radiation by factors of up to several hundred [78]. Kazuma et al. [106] showed that sites in $\mathrm{Ag}$ nanorods on $\mathrm{TiO}_{2}$ exposed to higher electromagnetic fields generate more hot electrons. After SPP excitation and hot electron generation, oxidized $\mathrm{Ag}^{+}$ ions diffuse into the water layer, adsorb on the $\mathrm{TiO}_{2}$ substrate, and eventually recombine with electrons from $\mathrm{TiO}_{2}$, leading to the formation of satellite redeposited $\mathrm{Ag}$ islands, as shown in Fig. 15 (top). 

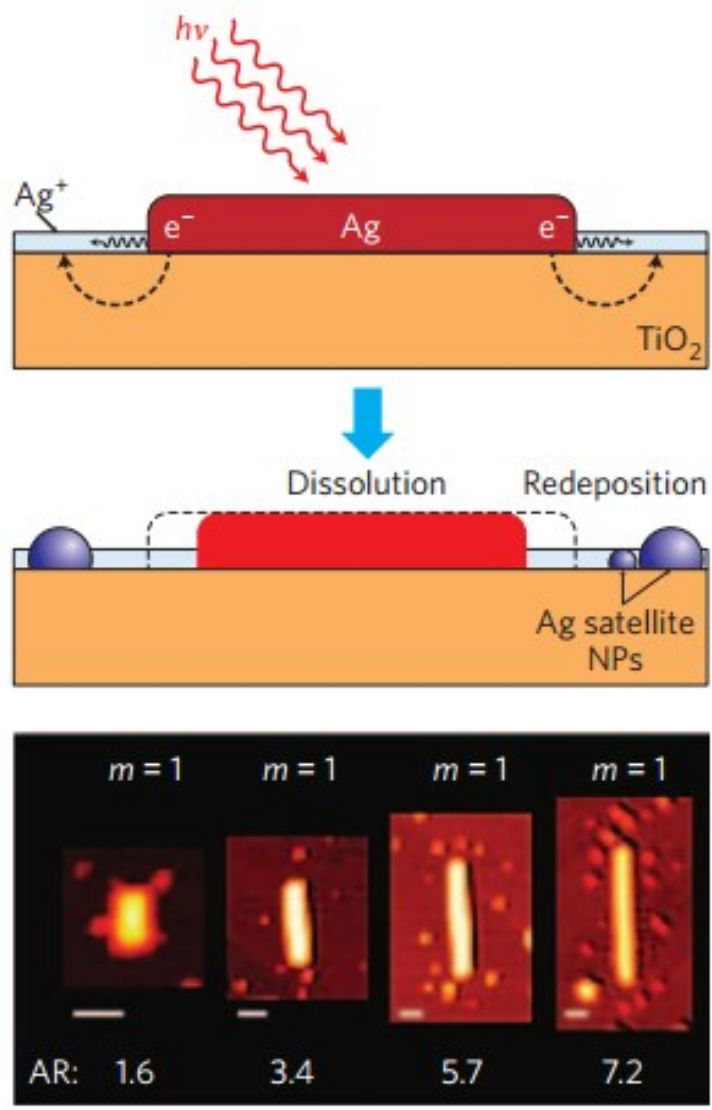

Fig. 15 (Adapted from [106]) Oxidation of Ag nanorods and redeposition of small Ag nanoparticles takes place as a result of plasmon-induced charge separation. After LSPR excitation and hot-electron generation, the oxidized $\mathrm{Ag}^{+}$ ions diffuse in the water layer adsorbed on the $\mathrm{TiO}_{2}$ substrate and eventually recombine with electrons from $\mathrm{TiO}_{2}$, forming satellite redeposited islands. The AFM images show Ag nanorods with various aspect ratios (ARs) that have undergone excitation of multipole plasmon modes from $m=1$ to 4 after irradiation with $800 \mathrm{~nm}$. The rods experience changes in length depending on the incident wavelength and mode excited. The horizontal scale bars are $50 \mathrm{~nm}$ in length.

This effect was observed in nanorods with different aspect ratios that sustained different multipole plasmon modes ( $m$ is $1,2,3,4$ ), as shown in the atomic force microscopy (AFM) images in the bottom of Fig. 15. Islands appeared in the regions where the SPP electromagnetic field was more intense, confirming the hypothesis that the charge generation process is induced and/or enhanced by the intense fields on the nanostructures. 


\subsubsection{Waveguide Detectors}

Schottky photodetectors based on IPE, integrated with a thin metal stripe waveguide in symmetric [17] and asymmetric [2] claddings have also been proposed, operating with long range and singleinterface SPPs, respectively. Fig. 16(a) shows a sketch of an asymmetric SPP waveguide photodetector consisting of a thin narrow metal stripe on Si with air on top [2]. Structures were fabricated as Au or Al stripes on n-Si forming Schottky contacts thereon. The $s a_{\mathrm{b}}{ }^{0}$ mode [107], localised to the metal-Si interface, was excited at wavelengths below the bandgap of Si via buttcoupling to a tapered polarisation-maintaining single mode fiber (PM-SMF). This mode propagates along the metal-Si interface with strong absorption, creating hot carriers in the metal along the Schottky contact, some of which cross over the Schottky barrier and are collected as the photocurrent. Fig. 16(b) presents the spectral response in Fowler form of a Au stripe of width 2.5 $\mu \mathrm{m}$ and thickness $135 \mathrm{~nm}$ on $\mathrm{n}-\mathrm{Si}$, for a reverse bias of $0.2 \mathrm{~V}$ and an incident optical power of 2 $\mathrm{mW}$ [2]. The intercept with the abscissa yields the cut-off photon energy, $\sim 0.765 \mathrm{eV}$, corresponding to a cut-off wavelength of $\lambda_{0} \sim 1620 \mathrm{~nm}$. Responsivities up to $1 \mathrm{~mA} / \mathrm{W}$ were reported with this photodetector schematic. Fig. 16(c) shows a measured photocurrent map of a Au on p-Si detector, generated by scanning a tapered PM-SMF over the end facet (following Fig. 16(a)) using a piezoelectric nanopositioner [37]. The Au stripe width and thickness in this case were $1.5 \mu \mathrm{m}$ and $40 \mathrm{~nm}$, the reverse bias was $0.1 \mathrm{~V}$, the incident optical power was $1 \mathrm{~mW}$, and the excitation wavelength was $1310 \mathrm{~nm}$. The photocurrent was largest when the PM-SMF was perfectly aligned with the stripe maximising the overlap between the incident beam and the $s a_{\mathrm{b}}{ }^{0}$ mode of the waveguide. 

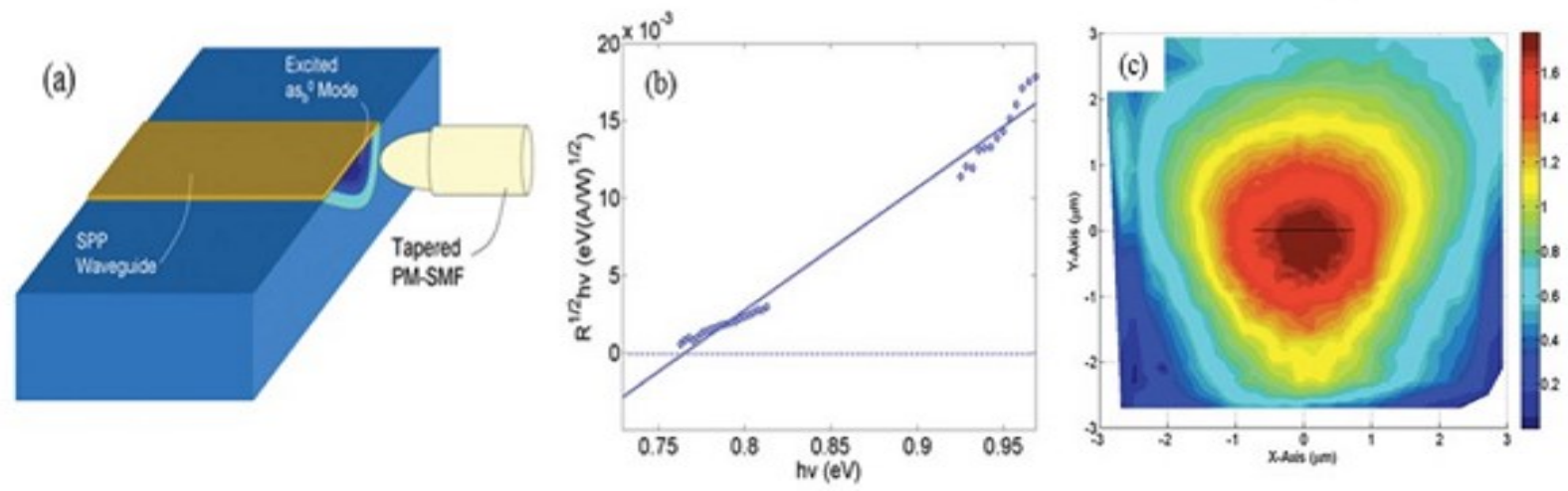

Fig. 16 (a) (Adapted from [37]) Sketch of an SPP waveguide detector consisting of a metal stripe forming a Schottky contact on Si, excited in the $s a_{\mathrm{b}}{ }^{0}$ mode [107] via butt-coupling to a tapered polarisation-maintaining single mode fibre (PM-SMF). (b) (Adapted from [2]) Spectral response of a Au on n-Si detector as sketched in (a), plotted in Fowler form. The Au stripe width and thickness were $2.5 \mu \mathrm{m}$ and $135 \mathrm{~nm}$, the reverse bias was $200 \mathrm{mV}$ and the incident optical power was $2 \mathrm{~mW}$. (c) (Adapted from [37].) Photocurrent map ( $\mu \mathrm{A})$ of a Au on p-Si detector as sketched in (a), generated by scanning a tapered PM-SMF over the end facet using a piezoelectric nanopositioner. The Au stripe width and thickness were $1.5 \mu \mathrm{m}$ and $40 \mathrm{~nm}$ (sketched as the black horizontal line), the reverse bias was $100 \mathrm{mV}$, the incident optical power was $1 \mathrm{~mW}$, and the excitation wavelength was $1310 \mathrm{~nm}$.

Other studies involving asymmetric metal stripe Schottky detectors include operation under a strong reverse bias approaching breakdown where a $10 \times$ increase in responsivity relative to low bias was reported [108], and a performance comparison of such detectors for two metals on p-Si and $\mathrm{n}-\mathrm{Si}$, two SPP modes of operation, and several stripe geometries [109].

\subsubsection{Optical Antenna Detectors}

The electric near-fields produced by nanoantennas can be of much greater intensity than the incident electric fields [41], therefore the photo-detection volume can shrink, leading potentially to faster devices with compelling signal to noise characteristics [110]. Mousavi et al. investigated a SPP photodetector concept, based on the enhancement of electrical near-field in low-defect, lowdoped $\operatorname{In}_{0.53} \mathrm{Ga}_{0.47} \mathrm{As}$ detection volumes located in the gaps of an array of metal nanodipole antennas [55]. Significant enhancement in responsivity in the presence of nanodipoles at telecom 
wavelengths is predicted $(\sim 100 \mathrm{~mA} / \mathrm{W}$ at $1550 \mathrm{~nm})$. The $3-\mathrm{dB}$ electrical bandwidth of the device is estimated based on its RC rise time and the hole transit time through the detection volume for the cases of conventional and ballistic transport in InGaAs and is found to range from $\sim 0.7$ to 4 THz. Knight et al. reported an active optical monopole array which can be considered as a highly compact, wavelength-specific, and polarization-specific light detector [60].

Desiatov et al. proposed a nanoscale broadband Si plasmonic Schottky photodetector with high responsivity and improved signal to noise ratio operating in the sub-bandgap regime [84]. Fig. 17 (a) and (b) show SEM images of a typical fabricated device [84]. The apex of pyramid was found to be $\sim 50 \mathrm{~nm}$. Due to the large cross-section of the pyramid, light is collected from a large area which corresponds to its base, concentrated toward the nanoapex of the pyramid, absorbed in the metal, and generates hot electrons. At the apex of the pyramid, the electric field intensity is enhanced by a factor of $\sim 30$ compared with the electromagnetic intensity in the base of the silicon pyramid. Theoretical investigation proves that most of the hot electrons are generated in the metallic part of the pyramid close to the pyramid's apex. Fig. 16 (c) shows the measured $I-V$ characteristic of a typical device for different wavelengths of illumination at a constant incident optical power. The responsivity of the device is found to be 5,12 , and $30 \mathrm{~mA} / \mathrm{W}$ for incident optical wavelengths of $1550 \mathrm{~nm}, 1300 \mathrm{~nm}$, and $1064 \mathrm{~nm}$, respectively. 

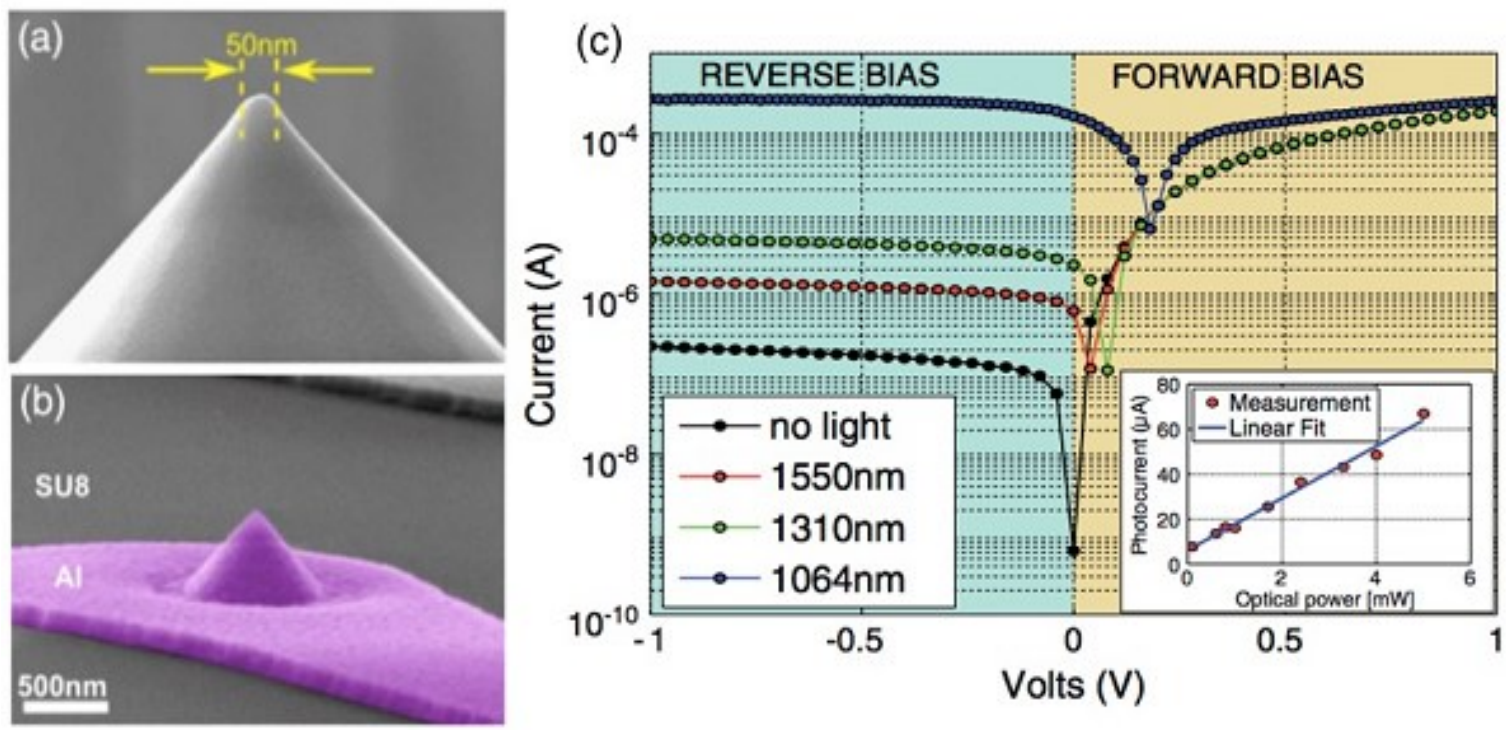

Fig. 17 (Adapted from [84]) SEM images of a plasmonic enhanced Si pyramids, (a) Formation of the nanoapex in the silicon pyramid. (b) SEM image of a final fabricated device, (c) $I-V$ measurements of the pyramid Schottky device at constant optical power for three different wavelengths and dark measurement. The inset shows the photocurrent versus optical power data and linear fit for $1550 \mathrm{~nm}$ wavelength.

Beside the ability in tunability of operating wavelength and bandwidth in SPP photodetectors, there are many interesting plasmonic structures which enable a straightforward tailoring of the polarization dependence. In the past decade, SPRs have been employed in creating metamaterials offering interesting optical properties such as strong chirality [111] which is some orders of magnitude higher than that of chiral molecules. Distinguishing between the two polarizations of circularly polarized light (CPL) is an inherent issue and a big problem with conventional photodetectors due to the fact that conventional semiconductors lack intrinsic chirality. Li et al. proposed a nanoantenna CPL photodetector in which the ability to distinguish left-handed circularly polarized (LCP) light and right-handed circularly polarized (RCP) light are obtained from the engineered chirality in metamaterial plasmonic nanostructures [112]. Fig. 18(a) shows a periodic array of chiral metamaterials consisting of a unit cell of Ag nanoantenna on top of a PMMA spacer and an optically thick layer of $\mathrm{Ag}$ as backplane forming a Schottky interface 
between Si and Ag nanowires. Generated hot electrons can then be captured and the high circular dichroism (CD) directly leads to enhanced discrimination between LCP and RCP in the CPL photodetectors. Fig. 18(b) and (c) show two fabricated (left-hand) LH and (right-hand) RH chiral metamaterials arrays and their theoretical and experimental responses. Responsivities are obtained by illuminating the metamaterials with a $\mathrm{CP}$ laser source and measuring photocurrent as a function of the laser handedness and wavelength. The peak value for responsivity is $\sim 2.2 \mathrm{~mA} / \mathrm{W}$ corresponding to a quantum efficiency of $\sim 0.2 \%$ which is two times that of chiral organic semiconductor transistors reported in [113].
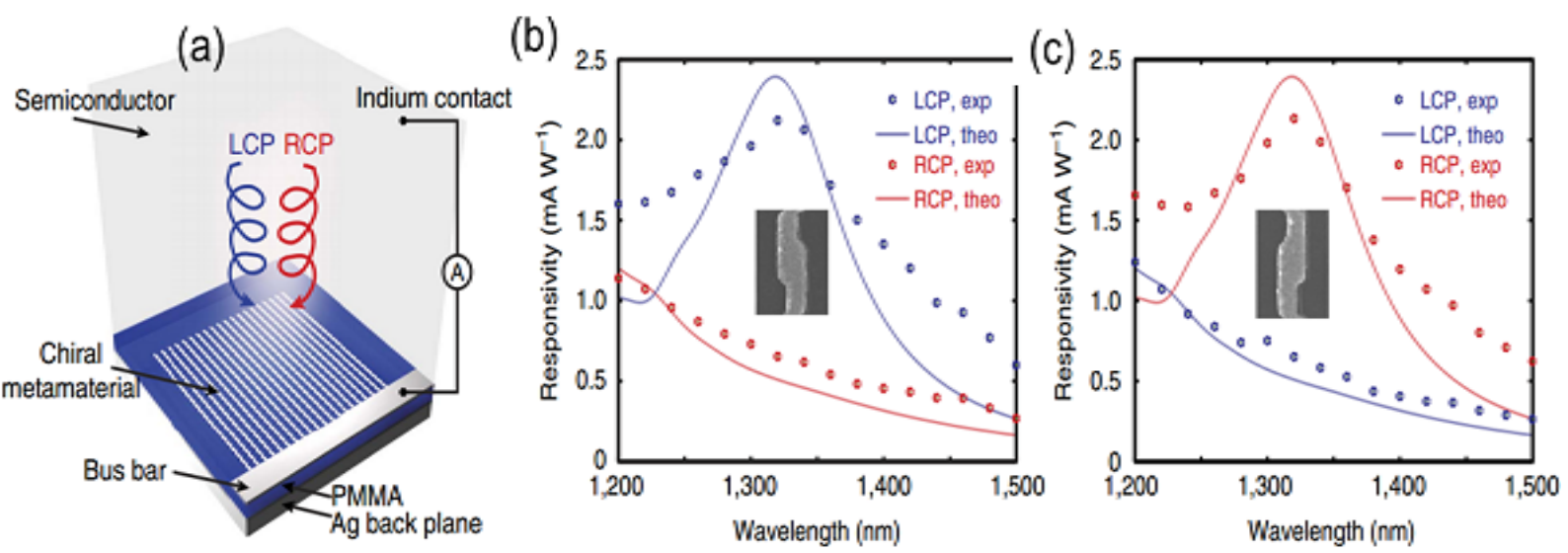

Fig. 18 (Adapted from [112]) (a) Schematic of the CPL detector consisting of a chiral metamaterial integrated with a semiconductor that serves as a hot electron acceptor. The Ohmic contact on $\mathrm{Si}$ is formed by soldering indium. The circuit is formed by wire bonding to the silver bus bar and indium, (b), (c) Photoresponsivity spectra of experimentally measured (dots) and theoretically calculated (solid curve) under LCP (blue) and RCP (red) illumination for LH (b) and RH (c) metamaterials.

One can combine this CPL photodetector with an existing linearly polarized IPE photodetectors and make a hot electron polarimeter with the ability to determine the Stokes parameters or the sates of polarization (SOP) of an arbitrarily polarized beam. 


\subsubsection{Theoretical Investigations and Proposals}

A complete review of the theory of IPE in Schottky junctions has been reported, and the Vickers model enriched by Casalino et al. [114]. The conventional Fowler equation used in the description of IPE was modified. They propose an analytical formulation to calculate the metal thickness that maximizes the efficiency of the devices. An important point addressed by this work is to determine if IPE-based $\mathrm{Si}$ devices have the potential to compare with photodetectors based on III-V compounds (e.g., GaAs and GaAsIn) and play a key role in telecommunications.

In general, plasmonic nanostructures are deposited on top of the semiconductor, which is convenient from the fabrication point of view but this causes only a small percentage of hot electrons to be excited with a wavevector permitting transport across the Schottky barrier. Embedding the nanostructure into the semiconductor introduces vertical Schottky interfaces, which permit emission of ballistic electrons over the Schottky barriers into the semiconductor, increasing the internal quantum efficiency. Scales and Berini presented phenomenological models for the internal quantum efficiency of Schottky barrier photodetectors suitable for the detection of optical radiation below the bandgap energy of the semiconductor based on IPE from the metal into the semiconductor substrate [68]. They studied both single and two Schottky barrier configurations. The double-barrier case provides higher enhancement due to emission over two barriers. The models proposed are based on assessing the emission probability of hot carriers as a function of their energy, taking into account multiple reflections within the metal film and energy losses due to internal scattering. Following this model, a Schottky contact detector comprising a symmetric metal stripe buried in p-type $\mathrm{Si}$, capable of detecting SPPs at wavelengths below the bandgap of $\mathrm{Si}$, is described in [17]. End-fire coupled responsivities of about $100 \mathrm{~A} / \mathrm{W}$ and minimum detectable powers of about $-20 \mathrm{dBm}$ are predicted at room temperature. They also 
comprehensively studied the internal quantum efficiency, responsivity, and sensitivity of symmetric surface-plasmon waveguide Schottky detectors, consisting of a thin metal stripe buried in Si. Responsivities of 0.1 to $0.21 \mathrm{~A} / \mathrm{W}$ and receiver sensitivities of -24 and $-18 \mathrm{dBm}$ are predicted for 1.5 and $10 \mathrm{GHz}$ electrical bandwidths ( 2.5 Gbit/s and >; $10 \mathrm{Gbit} / \mathrm{s})$, respectively.

Knight et al. determined that embedded nanowires, under normal incidence can produce a $25 \times$ greater efficiency than comparable planar Schottky devices [115]. This clearly suggests that 3D Schottky barriers can be a key design feature for increasing the efficiency of plasmon-based photodetection. A polarization-insensitive and high-efficiency plasmonic sub-bandgap Si Schottky photodetector operating at telecom wavelengths via IPE is proposed in [116]. The proposed structure, shown in Fig. 19(a), consists of a Si ridge waveguide, a Au Schottky electrode (of thickness $h_{\mathrm{Au}}$ ) covering both the top and sidewalls of the Si ridge, an aluminum (Al) Ohmic electrode on top of the Si slab, and a thin SU8 insulating film (of thickness $h_{\mathrm{SU}}$ ) separating the two electrodes. The hot carrier emission probability $P(\mathrm{E})$ is a very important factor in the estimation of the internal quantum efficiency. Depending on the configuration of the structure, the emission cone may consist of one of the sub-figures shown in Fig. 19(b). For the proposed detector, $P(\mathrm{E})$ for $\mathrm{Au}-\mathrm{Si}$ round interfaces follows the same expression as that for flat interfaces (See Fig. 19(b) top-right side one). By comparison with a diode with only a top Au contact, the polarization dependent responsivity is significantly minimized in this photodetector and higher responsivities for both TE and TM polarizations are achieved over a very broad wavelength range of 1.2-1.5 $\mu \mathrm{m}$.

Figs. 19(c) and (d) show the effects of $h_{\mathrm{Au}}$ on the responsivity $(R)$ and polarization dependent deviation (PDD) of the device. Specifically, changing $h_{\mathrm{Au}}$ changes the total absorption and the responsivity of the detector and also the polarization dependence of the responsivity. When $h_{\mathrm{Au}} \geq$ $50 \mathrm{~nm}$, the absorption spectrum no longer changes very much for both TE and TM polarizations, 
because the optical field is tightly confined to the $\mathrm{Au}-\mathrm{Si}$ interfaces and it can be sufficiently absorbed with a $50 \mathrm{~nm}$ thick Au film. However, PDD is very large over the whole wavelength range for $h_{\mathrm{Au}} \geq 50 \mathrm{~nm}$. As $h_{\mathrm{Au}}$ decreases, PDD decreases first and then quickly increases. At $h_{\mathrm{Au}}$ $=10 \mathrm{~nm}$, the internal quantum efficiency is the highest in each part of the Au film among all the cases.

(a)

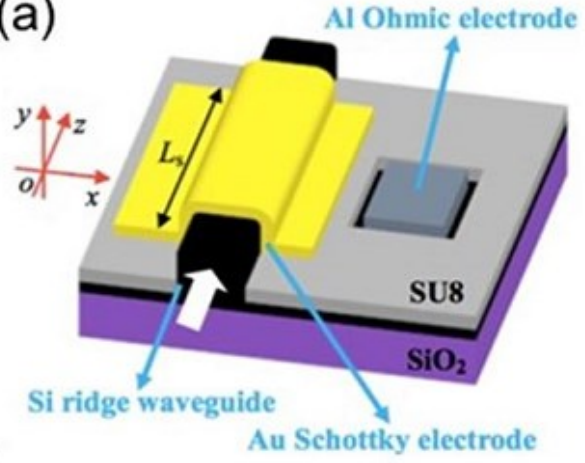

(b)
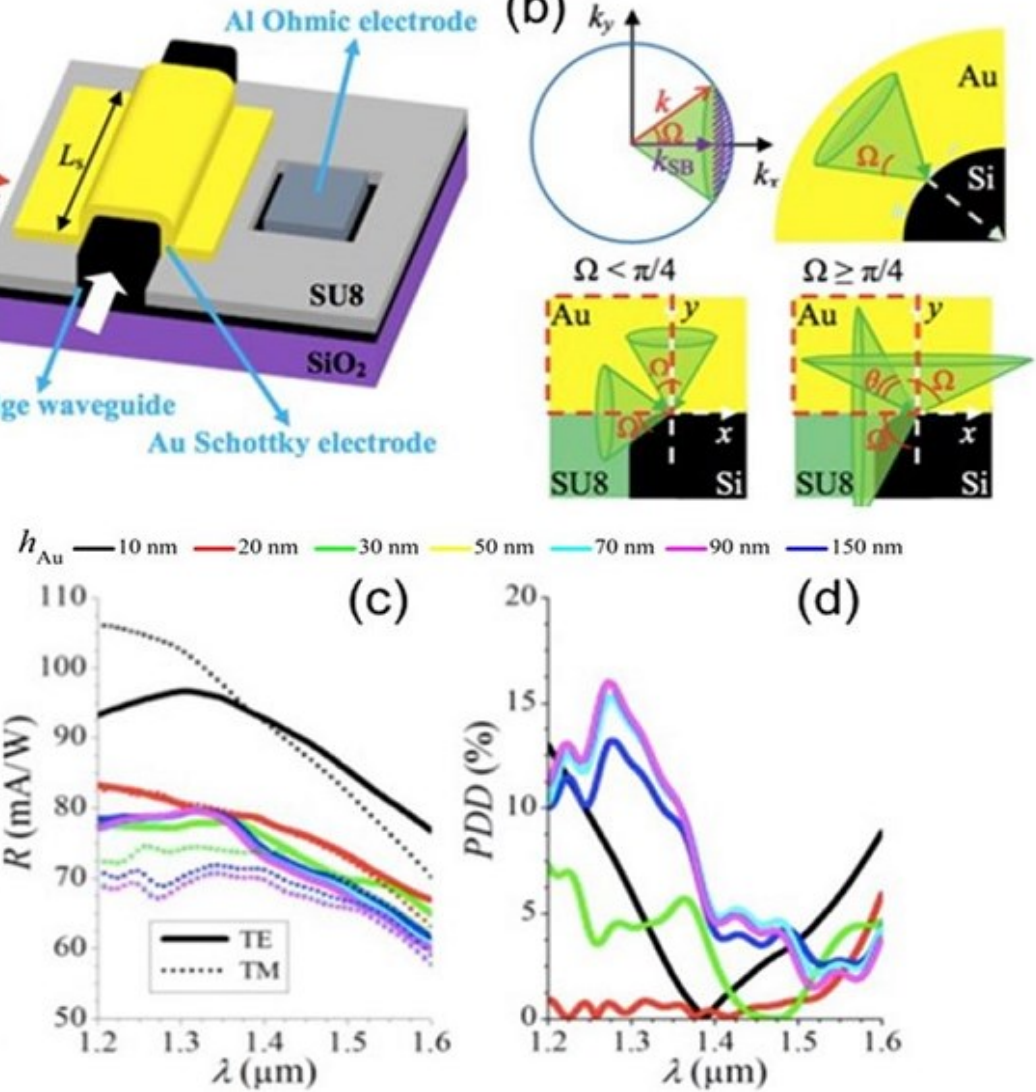

$50 \mathrm{~nm}-70 \mathrm{~nm}-90 \mathrm{~nm}-150 \mathrm{~nm}$

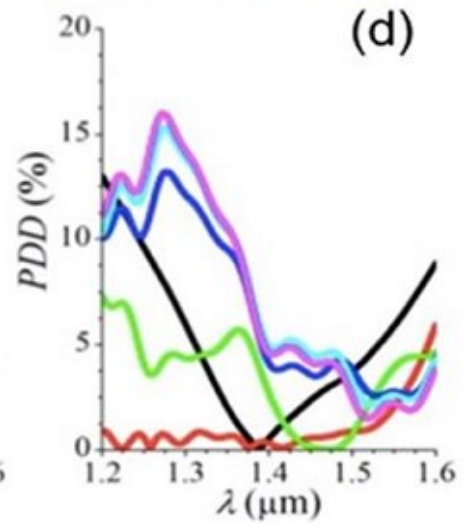

Fig. 19 (Adapted from [116]) (a) Three-dimensional schematic diagram of the proposed Si photodetector, (b)

Emission cones with angle, $\Omega$ : (top-left) a momentum-space illustration; (top-right) real-space illustrations for round Au-Si interfaces and (bottoms) an asymmetric corner denoted by the red dashed rectangles when (left) $\Omega \geq \pi / 4$ and (right) $\Omega<\pi / 4$, (c) responsivity, $R$, for both TE (solid lines) and TM (dotted lines) polarizations, and (d) polarization dependent deviation PDD of responsivity, for photodetectors with $h_{\mathrm{Au}}=10,20,30,50,70$, and $90 \mathrm{~nm}$. The SU8 film thickness is $h_{\mathrm{SU} 8}=60 \mathrm{~nm}$. 
Therefore, the responsivity spectrum rises to the highest level, especially in the short wavelength range, even though the absorption spectrum lies in the same range as the other cases with larger $h_{\mathrm{Au}}$. In this case, the internal quantum efficiency dominates the responsivity spectrum.

\subsection{Summary}

This review highlights opportunities for the generation and use of plasmon-induced hot carriers in metallic nanostructures on semiconductors. EHP creation and IPE have both been used extensively as detection mechanisms. Interest in IPE is motivated by the fact that it enables sub-bandgap detection so the optical bandwidth of a detector can be extended to photon energies below the bandgap of the semiconductor. IPE-based SPP photodetectors typically combine a metallic structure that supports SPPs, such as a planar or grating structure, metallic nano-particles, or holes in metal films, with a semiconductor detector structure such as a Schottky or pn junction. Several investigations aim at improving the efficiency of detectors based on IPE by exciting SPPs along a metal contact forming the device. Target applications of IPE are very broad, but improving the efficiency of solar cells and providing infrared detection at telecom wavelengths in $\mathrm{Si}$ are frequently evoked.

Although performance and reliability of SPP-induced hot carrier generation has been comprehensively proposed and observed in many works highlighted in this review paper, further fundamental studies are needed to optimize these processes for viable technological applications. Particularly, it will be important to determine more precisely the timescales involved in hot carrier relaxation and what physical and chemical parameters influence these timescales. Properties inherent to SPPs are exploited to convey useful characteristics to detectors in addressing applications such as low-noise high-speed detection, single-plasmon detection, near and mid- 
infrared imaging, solar energy conversion and (bio)chemical sensing. The prospects for combining SPPs with detection structures are bright.

Finally, it is worth to summarize some of described photodetector architectures involving SPPs and LSPRs that appear particularly promising as follows:

Gratings: Early investigations were based on grating-coupled detector structures. Corrugated grating couplers were used initially to create integrated and compact structures which are of high interest today. Table 1 briefly reviews the structures and characteristics of the devices explained in this paper:

Table 1 Characteristics of some grating-based photodetectors

\begin{tabular}{cccccc}
\hline Reference & Structure / Material & $\begin{array}{c}\text { R @ zero bias } \\
(\mathbf{m A} / \mathbf{W})\end{array}$ & $\begin{array}{c}\text { Optical BW } \\
\mathbf{( T H z )}\end{array}$ & $\begin{array}{c}\text { Electrical } \\
\text { BW (GHz) }\end{array}$ & $\begin{array}{c}\text { Wavelength } \\
(\mathbf{n m})\end{array}$ \\
\hline$[25]$ & Disk arrays / Au/Si & 0.0375 & NA & NA & $1500-1600$ \\
\hline$[58]$ & Nano-slit / Au/Ti/Si & 0.6 & 13 & NA & $1290-1635$ \\
\hline$[24]$ & Nano-slit, ring/ & 0.038 & NA & NA & $1500-1600$ \\
\hline$[90]$ & $\mathrm{Au} / \mathrm{Si}$ & & & & $1520-1620$ \\
\hline
\end{tabular}

Other interesting similar structures can be found in [34], [48], [86], [87], [88] and [89].

MIM, MOM, MSM and MIS: Referring to this point that in modern optical communication systems, size, responsivity, dark current and photocurrent of a detector are very important, these kinds of detector structures are popular for high-speed operation because the contact architecture lowers the capacitance and carrier transit times. It is promising to design the contacts in such a detector simultaneously as a nanowire grating to couple incident light to SPPs thereon, such that they overlap strongly with the detection region. Table 2 briefly reviews the structures and characteristics of the devices explained in this paper: 
Table 2 Characteristics of some MIM and MSM photodetectors

\begin{tabular}{|c|c|c|c|c|c|}
\hline Reference & Structure / Material & $\begin{array}{c}\text { R@zero bias } \\
(\mathbf{m A} / \mathbf{W})\end{array}$ & $\begin{array}{c}\text { Optical BW } \\
\text { (THz) }\end{array}$ & $\begin{array}{l}\text { Electrical } \\
\text { BW (GHz) }\end{array}$ & $\begin{array}{c}\text { Wavelength } \\
\text { (nm) }\end{array}$ \\
\hline$[93]$ & $\mathrm{MIM} / \mathrm{Au} / \mathrm{Al}_{2} \mathrm{O}_{3} / \mathrm{Au}$ & 0.55 & NA & NA & $400-700$ \\
\hline$[94]$ & $\mathrm{MIM} / \mathrm{Ag} / \mathrm{TiO}_{2} / \mathrm{Ag}$ & 0.1 & 1.13 & $\mathrm{NA}$ & $\sim 1550$ \\
\hline [95] & $\mathrm{MSM} / \mathrm{Cu} / \mathrm{SOI} / \mathrm{Al}$ & 0.01 & NA & 1 & $\sim 1550$ \\
\hline
\end{tabular}

Other interesting similar structures can be found in [30], [91], [92] and [97].

Nanoparticles: As light strikes metal nanoparticles, SPP resonances can be excited thereon, high near-field enhancement factors can be generated, and strong absorption or scattering leading to light trapping can occur. These effects are useful to increase the absorptance of detectors, particularly those based on a thin absorption layer, or at wavelengths where the absorption is low. These effects are promising to improve the performance of thin-film Si or organic solar cells with application to photovoltaic and catalytic devices. Table 3 briefly reviews the structures and characteristics of the devices explained in this paper:

Table 3 Characteristics of some nanoparticles-based photodetectors

\begin{tabular}{|c|c|c|c|c|c|}
\hline Reference & Structure / Material & $\begin{array}{c}\mathbf{R} \text { @ zero bias } \\
(\mathbf{m A} / \mathbf{W})\end{array}$ & $\begin{array}{c}\text { Optical BW } \\
\text { (THz) }\end{array}$ & $\begin{array}{l}\text { Electrical } \\
\text { BW (GHz) }\end{array}$ & $\begin{array}{c}\text { Wavelength } \\
\text { (nm) }\end{array}$ \\
\hline$[12]$ & Nanoislands / Au/Si & 2 & 150 & NA & $1250-1550$ \\
\hline [99] & Nanoislands / $\mathrm{Au} / \mathrm{TiO}_{2}$ & NA & 3480 & NA & $600-630$ \\
\hline$[103]$ & Decorated / Ag/TiO2 & NA & 3000 & NA & $640-700$ \\
\hline$[105]$ & Flower-rods/ $\mathrm{Au} / \mathrm{ZnO}$ & NA & 3150 & NA & $500-600$ \\
\hline
\end{tabular}


Other interesting similar structures can be found in [117], [100], [101], [102] and [104]

Waveguides: SPP waveguides can be formed into SPP photodetectors by replacing a dielectric region with a detection medium. Such detectors are attractive because they can be integrated with other plasmonic or photonic waveguides, they can increase the absorptance, shrink dimensions, enhance the detection process by exploiting enhanced or localised guided fields, and provide broad electrical and optical bandwidths. Table 4 briefly reviews the structures and characteristics of the devices explained in this paper:

Table 4 Characteristics of some waveguide-based photodetectors

\begin{tabular}{|c|c|c|c|c|c|}
\hline Reference & Structure / Material & $\begin{array}{c}\text { R@zero } \\
\text { bias } \\
(\mathrm{mA} / \mathbf{W})\end{array}$ & $\begin{array}{c}\text { Optical } \\
\text { BW (THz) }\end{array}$ & $\begin{array}{l}\text { Electrical } \\
\text { BW (GHz) }\end{array}$ & $\begin{array}{c}\text { Wavelength } \\
(\mathbf{n m})\end{array}$ \\
\hline$[37]$ & Asymmetric metal stripe / Au/p-Si & 0.95 & NA & NA & $1300-1620$ \\
\hline [109] & $\begin{array}{c}\text { Asymmetric metal stripe / Au/n- } \\
\qquad \mathrm{Si}, \mathrm{Al} / \mathrm{n}-\mathrm{Si}\end{array}$ & 0.25 & NA & NA & $1300-1620$ \\
\hline
\end{tabular}

Other interesting similar structures can be found in [2] and [17], [107] and [108].

Nanoantennas: Optical nanoantennas are passive structures that capture part of an incident optical wave to produce oscillating near-fields at defined locations along its geometry. Combining this function with photodetection leads to integrated nanoscale photodetectors having compelling characteristics, as the antennas allow the detection volume to shrink, leading to fast devices with high signal-to-noise characteristics, and to polarisation-resolved spectroscopic nano-detectors. Such properties have attracted much interest lately, due to the implications they have both in fundamental research and in technological applications. Table 5 briefly reviews the structures and characteristics of the devices explained in this paper:

Table 5 Characteristics of some nanoantennas-based photodetectors 


\begin{tabular}{|c|c|c|c|c|c|}
\hline Reference & Structure / Material & $\begin{array}{c}\text { R@zero } \\
\text { bias } \\
(\mathrm{mA} / \mathbf{W})\end{array}$ & $\begin{array}{c}\text { Optical } \\
\text { BW (THz) }\end{array}$ & $\begin{array}{l}\text { Electrical } \\
\text { BW (GHz) }\end{array}$ & $\begin{array}{c}\text { Wavelength } \\
\text { (nm) }\end{array}$ \\
\hline 24 & $\begin{array}{l}\text { Nanodipole array / } \\
\mathrm{Au} / \mathrm{In}_{0.53} \mathrm{Ga}_{0.47} \mathrm{As} / \mathrm{InP}\end{array}$ & 100 & 3300 & 4 & $1520-1600$ \\
\hline 55 & Nanopyramids / Al/Si & 30 & NA & NA & $1000-1200$ \\
\hline 87 & $\begin{array}{c}\text { Nanochiral / } \\
\text { Metamaterial/PMMA/Ag }\end{array}$ & 2.2 & 2000 & NA & $1300-1500$ \\
\hline
\end{tabular}

Other interesting similar structures can be found in [41], [110] and [111].

Authors' note: This review article comprehensively surveys and compares the most recent cutting-edge surface plasmon-polaritons IPE-based photodetectors reported until mid-2016. From the time of submission of the paper to now, more research with promising results has been reported in the literature in this topic which we did not address them yet. In terms of responsivity which is the main interest of this chapter, it has been shown in [118] that the insertion of a graphene monolayer in between the metal and the semiconductor greatly enhances the internal quantum efficiency to about $7 \%$ and responsivity to the record number (at the time of writing this thesis) of $85 \mathrm{~mA} / \mathrm{W}$. At a reverse bias of $3 \mathrm{~V}$, they achieve avalanche multiplication, with $0.37 \mathrm{~A} / \mathrm{W}$ responsivity and avalanche photo-gain $\sim 2$. 


\section{Chapter: Design, Fabrication and Measurement of a High-}

\section{Responsivity Sub-Bandgap Hot-Hole Plasmonic Schottky Detectors}

The following article entitled "High-responsivity sub-bandgap hot-hole plasmonic Schottky detectors" was published in Optics Express, Volume 24, Issue 20 on Oct. 2016 and is presented in full. Fabrication of gratings are done in Carleton University Fabrication Lab and Berini Lab at the University of Ottawa under supervision of Anthony Olivieri. Numerical simulation, AFM and the experimental results are solely done by the primary author under the supervision and guidance of the co-authors. The characterization was performed entirely at the Berini Lab at the University of Ottawa.

In this manuscript, we propose a novel sub-bandgap photodetector consisting of a metal grating on a thin metal patch on silicon, which makes use of the enhancement produced by the excitation of surface plasmon polaritons at the metal-silicon interface. The grating is defined via e-beam lithography and Au lift-off on an Au patch defined beforehand by optical lithography on doped p-type silicon. The surface plasmon polaritons are absorbed by the metal, leading to the creation of hot holes that can cross into the silicon where they are collected as the photocurrent. We report infrared responsivities among the largest achieved to date. Given the small area of each detector, thousands can be densely integrated into arrays over the surface of the substrate. The Schottky photodetector can be used in, e.g., non-contact wafer probing or in short-reach optical 
communications applications. The experiments were performed at the Berini Lab at the Centre for Research in Photonics in the University of Ottawa.

Some comments received from the examination board during the $\mathrm{PhD}$ thesis defense are carefully addressed in this preamble:

1- Our definition for p-polarised and s-polarised light: it is quite common to talk about spolarised and p-polarized laser beams, incident on optical surfaces or diffraction gratings. Usually, p-polarized light is understood to have an electric field direction parallel to the plane of incidence on a device, and s-polarised light has the electric field oriented perpendicular to that plane. (in fact, the letters have a German origin: $\mathrm{s}=$ senkrecht $=$ perpendicular, $\mathrm{p}=$ parallel.) According to that definition, we can obtain vanishing reflectivity of a surface at Brewster's angle for p-polarization. That's easy to remember: " $\mathrm{p}$ " is closer to "B" (like "Brewster"), so Brewster's trick works with $\mathrm{p}$ polarization. In the context of diffraction gratings, the opposite definition can be found in the literature. Here, "s" still means perpendicular, but in that case perpendicular not to the plane of incidence, but to the lines of the grating. So for s polarization, the electric field vector is perpendicular to the lines, i.e., it lies in the plane of incidence. Notably, laser beam is sometimes called s-polarised, when its polarization is vertical, i.e., perpendicular to the lab's floor. It is better, though, to restrict the terms $s$ 
polarization and $p$ polarization to cases where the polarization direction with respect to a plane of incidence is relevant.

2- Schottky barrier height, relative to the Fermi level is denoted by $\Phi_{\mathrm{B}}$ in $\mathrm{eV}$ throughout this paper. It may be used as $q \Phi_{\mathrm{B}}$ (in $\mathrm{eV}$ ) in the next chapters.

3- Throughout the paper, during the design of each structure using FDTD method, we did consider frequency dependency of any material in use. This point is not explained in the papers, instead we mentioned the database we have applied to the method.

4- Energy bandgap diagram in Fig. 1 is not correct in the published paper, $h v$ energy arrow on energy band diagram must be smaller than $E_{\mathrm{g}}$. Here we fixed the picture:

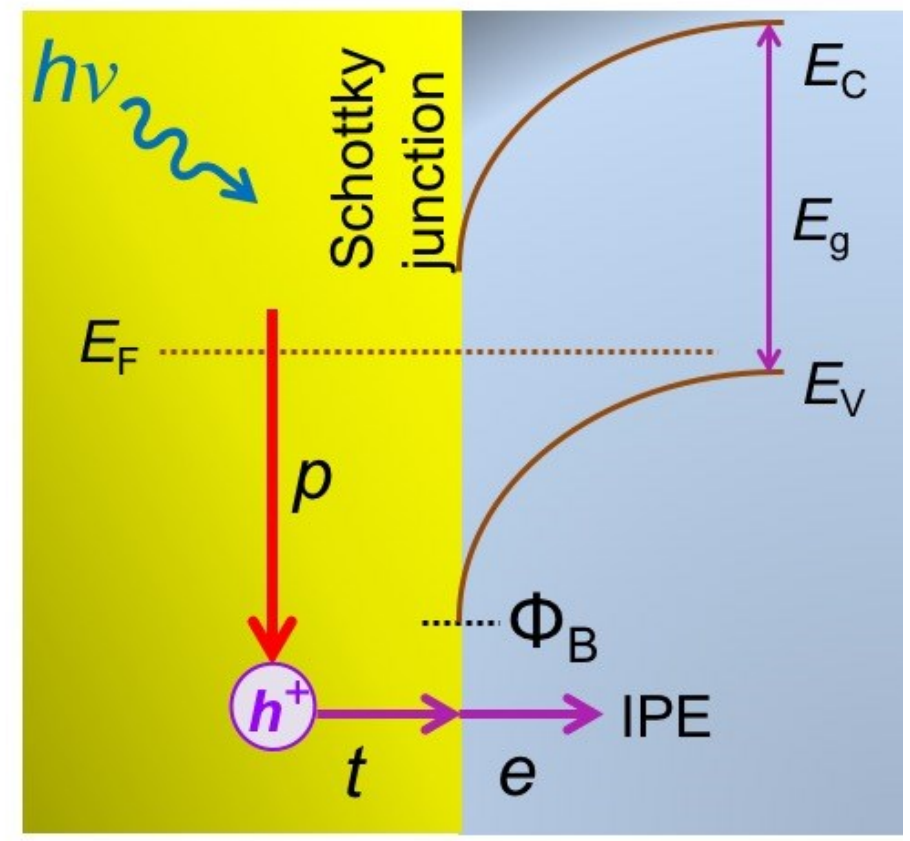




\title{
High-responsivity sub-bandgap hot-hole plasmonic Schottky detectors
}

\author{
Mohammad Alavirad, ${ }^{1,2}$ Anthony Olivieri, ${ }^{2}$ LANGis Roy, ${ }^{3}$ And Pierre \\ BERINI ${ }^{2,4,5, *}$ \\ ${ }^{I}$ Department of Electronics, Carleton University, 1125 Colonel By Drive, Ottawa, ON K1S 5B6, \\ Canada \\ ${ }^{2}$ Centre for Research in Photonics at the University of Ottawa, 800 King Edward Avenue, Ottawa, ON \\ K1N 6N5, Canada \\ ${ }^{3}$ Department of Electrical, Computer and Software Engineering, University of Ontario Institute of \\ Technology, 2000 Simcoe Street North Oshawa, ON L1H 7K4, Canada \\ ${ }^{4}$ Department of Electrical Engineering and Computer Science, University of Ottawa, 800 King Edward \\ Avenue, Ottawa, ON K1N 6N5, Canada \\ ${ }^{5}$ Department of Physics, University of Ottawa, 150 Louis Pasteur, Ottawa, ON K1N 6N5, Canada \\ berini@eecs.uottawa.ca
}

\begin{abstract}
In this paper we present a sub-bandgap photodetector consisting of a metal grating on a thin metal patch on silicon, which makes use of the enhancement produced by the excitation of surface plasmon polaritons at the metal-silicon interface. The grating is defined via e-beam lithography and Au lift-off on a Au patch defined beforehand by optical lithography on doped p-type silicon. The surface plasmon polaritons are absorbed by the metal, leading to the creation of hot holes that can cross into the silicon where they are collected as the photocurrent. Physical characterization of intermediate structure is provided along with responsivity measurements at telecom wavelengths. Results are promising in terms of responsivity, with a value of $13 \mathrm{~mA} / \mathrm{W}$ measured at $1550 \mathrm{~nm}$ - this is among the highest values reported to date for sub-bandgap detectors based on internal photoemission. The Schottky photodetector can be used in, e.g., non-contact wafer probing or in short-reach optical communications applications.
\end{abstract}

(C)2016 Optical Society of America

OCIS codes: (220.0220) Optical design and fabrication; (230.0230) Optical devices; (240.6680) Surface plasmons; (250.5403) Plasmonics; (350.2770) Gratings.

\section{References and links}

1. S. A. Maier, Plasmonics: Fundamentals and Applications (Springer Science + Business Media, 2007).

2. P. Berini, "Long-range surface plasmon polaritons," Adv. Opt. Photonics 1(3), 484-588 (2009).

3. M. Casalino, G. Coppola, M. Iodice, I. Rendina, and L. Sirleto, "Critically coupled silicon Fabry-Perot photodetectors based on the internal photoemission effect at $1550 \mathrm{~nm}$," Opt. Express 20(11), 12599-12609 (2012).

4. R. Sundararaman, P. Narang, A. S. Jermyn, W. A. Goddard 3rd, and H. A. Atwater, "Theoretical predictions for hot-carrier generation from surface plasmon decay," Nat. Commun. 5, 5788 (2014).

5. I. Goykhman, B. Desiatov, J. Shappir, J. B. Khurgin, and U. Levy, "Model for quantum efficiency of guided mode plasmonic enhanced silicon Schottky detectors," arXiv: 1401.2624 (2014).

6. M. L. Brongersma, N. J. Halas, and P. Nordlander, "Plasmon-induced hot carrier science and technology," Nat. Nanotechnol. 10(1), 25-34 (2015).

7. P. Berini, "Surface plasmon photodetectors and their applications," Laser Photonics Rev. 8(2), 197-220 (2014).

8. C. Clavero, "Plasmon-induced hot-electron generation at nanoparticle/metaloxide interfaces for photovoltaic and photocatalytic devices," Nat. Photonics 8(2), 95-103 (2014).

9. M. Tsuneyasu, C. Sasakawa, N. Naruishi, Y. Tanaka, Y. Yoshida, and K. Taw, "Sensitive detection of interleukin- 6 on a plasmonic chip by grating-coupled surface-plasmon-field-enhanced fluorescence imaging," Jpn. J. Appl. Phys. 53, 06JL05 (2014).

10. A. Takeda, T. Aihara, M. Fukuhara, Y. Ishii, and M. Fukuda, "Schottky-type surface plasmon detector with nano-slit grating using enhanced resonant optical transmission," J. Appl. Phys. 116(8), 084313 (2014).

11. A. Akbari, R. N. Tait, and P. Berini, "Surface plasmon waveguide Schottky detector," Opt. Express 18(8), 85058514 (2010). 
12. I. Goykhman, B. Desiatov, J. Khurgin, J. Shappir, and U. Levy, "Waveguide based compact silicon Schottky photodetector with enhanced responsivity in the telecom spectral band," Opt. Express 20(27), 28594-28602 (2012).

13. M. W. Knight, H. Sobhani, P. Nordlander, and N. J. Halas, "Photodetection with active optical antennas," Science 332(6030), 702-704 (2011).

14. X. Li, D. Xiao, and Z. Zhang, "Landau damping of quantum plasmons in metal nanostructures," New J. Phys. 15(2), 023011 (2013).

15. B. Desiatov, I. Goykhman, N. Mazurski, J. O. Shappir, J. B. Khurgin, and U. Levy, "Plasmonic enhanced silicon pyramids for internal photoemission Schottky detectors in the near-infrared regime," Optica 2(4), 335-338 (2015).

16. M. A. Nazirzadeh, F. B. Atar, B. B. Turgut, and A. K. Okyay, "Random sized plasmonic nanoantennas on Silicon for low-cost broad-band near-infrared photodetection," Sci. Rep. 4, 7103 (2014).

17. M. Alavirad, S. S. Mousavi, L. Roy, and P. Berini, "Schottky-contact plasmonic dipole rectenna concept for biosensing," Opt. Express 21(4), 4328-4347 (2013).

18. A. Sellai and P. Dawson, "Quantum efficiency in GaAs Schottky photodetectors with enhancement due to surface plasmon excitations," Solid-State Electron. 46(1), 29-33 (2002).

19. J. W. Yoon, W. J. Park, K. J. Lee, S. H. Song, and R. Magnusson, "Surface-plasmon mediated total absorption of light into silicon," Opt. Express 19(21), 20673-20680 (2011).

20. X. G. Guo, R. Zhang, J. C. Cao, and H. Liu, "Surface plasmon-enhanced absorption in metal grating coupled terahertz quantum well photodetectors," IEEE J. Quantum Electron. 48(9), 1113-1119 (2012).

21. J. Hetterich, G. Bastian, N. A. Gippius, S. G. Tikhodeev, G. von Plessen, and U. Lemmer, "Optimized design of plasmonic MSM photodetector," IEEE J. Quantum Electron. 43(10), 855-859 (2007).

22. T. Tekin, "Silicon photonic circuits: on-CMOS integration, fiber optical coupling, and packaging," IEEE J. Sel. Top. Quantum Electron. 17, 704-719 (2011).

23. M. Fukuhara, M. Ota, H. Sakai, T. Aihara, Y. Ishii, and M. Fukuda, "Low-loss waveguiding and detecting structure for surface plasmon polaritons," Appl. Phys. Lett. 104(8), 081111 (2014).

24. A. Olivieri, C. Chen, S. Hassan, E. Lisicka-Skrzek, R. N. Tait, and P. Berini, "Plasmonic nanostructured metaloxide-semiconductor reflection modulators," Nano Lett. 15(4), 2304-2311 (2015).

25. H. Hu, X. Zeng, D. Ji, L. Zhu, and Q. Gan, "Efficient end-fire coupling of surface plasmons on flat metal surfaces for improved plasmonic Mach-Zehnder interferometer," J. Appl. Phys. 113(5), 053101 (2013).

26. R. Hunsperger, Integrated Optics, Theory and Technology (Springer Science + Business Media, 2009).

27. S. M. Sze and K. K. Ng, Physics of Semiconductor Devices (Wiley, 2006).

28. FDTD Solutions; Lumerical Solutions Inc.: Vancouver, Canada, (Accessed 2014).

29. B. Hecht, H. Bielefeldt, L. Novotny, Y. Inouye, and D. W. Pohl, "Local excitation, scattering, and interference of surface plasmons," Phys. Rev. Lett. 77(9), 1889-1892 (1996).

30. C. Scales and P. Berini, "Thin-film Schottky barrier photodetector models," IEEE J. Quantum Electron. 46(5), 633-643 (2010).

31. P. Berini, A. Olivieri, and C. Chen, "Thin Au surface plasmon waveguide Schottky detectors on p-Si," Nanotechnology 23(44), 444011 (2012).

32. e_LiNE; Raith nanofabrication: Dortmund Germany, www.raith.com (Accessed 2014).

33. S. Hassan, E. Lisicka-Skrzek, A. Olivieri, R. N. Tait, and P. Berini, "Fabrication of a plasmonic modulator incorporating an overlaid grating coupler," Nanotechnology 25(49), 495202 (2014).

34. LabVIEW; National Instruments Corporation: Austin TX, United States, (Accessed 2014).

35. P. Kramer and L. J. van Ruyven, "Position of the band edges of silicon under uniaxial stress," Appl. Phys. Lett. 20(11), 420-422 (1972).

36. C. D. Lien, F. C. T. So, and M. A. Nicolet, "An improved forward I-V method for nonideal Schottky diodes with high sense resistance," IEEE Trans. Electron Dev. 31(10), 1502-1503 (1984).

37. J. H. Werner, "Schottky barrier and pn-junction I/V plots -Small signal evaluation," Appl. Phys., A Mater. Sci. Process. 47(3), 291-300 (1988).

38. C. C. Katsidis and D. I. Siapkas, "General transfer-matrix method for optical multilayer systems with coherent, partially coherent, and incoherent interference," Appl. Opt. 41(19), 3978-3987 (2002).

39. B. E. A. Saleh and M. C. Teich, Fundamentals of Photonics (Wiley, 2007).

40. S. M. Sze, C. R. Crowell, and D. J. Kahng, "Photoelectric determination of the image force dielectric constant for hot electrons in Schottky barriers," Appl. Phys. (Berl.) 35(8), 2534 (1964).

41. J. J. Ackert, D. J. Thomson, L. Shen, A. C. Peacock, P. E. Jessop, G. T. Reed, G. Z. Mashanovich, and A. P. Knights, "High-speed detection at two micrometres with monolithic silicon photodiodes," Nat. Photonics 9(6), 393-396 (2015).

\section{Introduction}

Nanometallic structures are key to the conversion of light to surface plasmon-polaritons (SPPs) localized to small volumes. They are promising for several applications including telecommunications, solar cells and biosensing as they can provide highly enhanced fields, strong confinement (to sub-wavelength scales) and high bulk and surface sensitivities [1, 2]. 
A Schottky barrier photodetector formed at the interface between a metal and a lightly doped semiconductor can be used to detect radiation below the bandgap energy of the semiconductor via internal photoemission (IPE) [3-5]. Several Schottky photodetectors involving SPP excitations on the metal contact have been reported [6-8], including gratingcoupled detectors $[9,10]$, waveguides [11, 12], and nanoparticles and nanoantennas [13-17], building on early work involving prism-coupling to SPPs on a suitable detection structure [18]. A grating structure can be used as an alternative to a prism to increase the in-plane momentum of the incident light in order to match that of the SPP, providing compactness and manufacturing advantages, and, potentially, high sensitivity. Metal-semiconductor Schottky grating structures have been investigated as SPP photodetectors $[19,20]$ and optimized for slit pitch and film thickness [10,21].

Although studies have been carried out on various aspects of gratings and their detection applications, achieving high infrared sub-bandgap responsivities over a broad spectral range on low-cost Si remains a difficult and worthwhile challenge. In this paper we propose a novel plasmonic Schottky photodetector consisting of a Au grating on a thin metal patch on p-type $\mathrm{Si}(\mathrm{p}-\mathrm{Si})$, providing detection via IPE of hot holes generated in the patch directly along the Schottky contact. We report infrared responsivities among the largest achieved to date. Given the small area of each detector, thousands can be densely integrated into arrays over the surface of the substrate. Packaging is simplified because butt-coupling and propagation through end-facets are not required, and simpler packages suitable for surface devices can be used [22]. Potential applications include infrared imaging, biosensors and optical receivers for interconnects [23, 24] and short-reach communications [25].

\section{Proposed Structure}

Figure 1(a) shows a schematic of the proposed structure. It includes a metal patch (thickness $t$ ) for guiding SPPs on a lightly-doped $\mathrm{p}-\mathrm{Si}$ epitaxial layer on a heavily doped $\mathrm{p}-\mathrm{Si}$ substrate. A metal grating on the metal patch is designed to couple normally-incident $p$-polarised infrared ( $\lambda_{0} \sim 1550 \mathrm{~nm}$, sub-bandgap) light to SPPs propagating along the bottom surface of the patch such that the SPP fields are coincident with the Schottky contact. As SPPs propagate they are absorbed in the metal, generating hot carriers therein primarily along the Schottky contact. Detection then occurs through the collection of hot holes in $\mathrm{p}-\mathrm{Si}$ via IPE. The substrate is placed on a backside $\mathrm{Al} \mathrm{Ohmic} \mathrm{ground} \mathrm{contact.}$
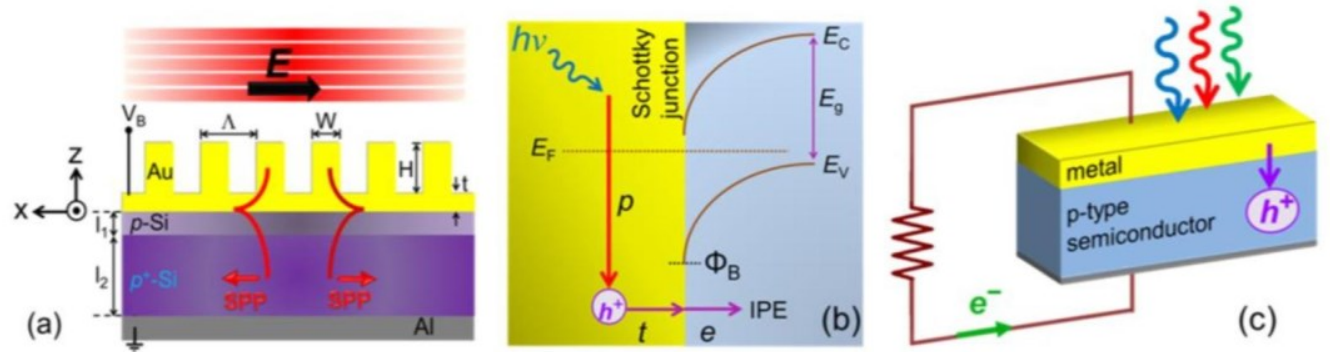

Fig. 1. (a) Cross-sectional sketch of the proposed $\mathrm{Au} / \mathrm{Si}$ Schottky surface plasmon photodetector. The structure comprises a metal patch of thickness $t$ on $\mathrm{p}-\mathrm{Si}$, with a metal grating consisting of rectangular ridges of width $W$ and thickness $H$ arranged periodically in pitch $\Lambda$. The materials considered are Au for the metal, $\mathrm{p}-\mathrm{Si}$ for the epitaxial layer and $\mathrm{p}^{+}-\mathrm{Si}$ for the substrate. The device is illuminated from the top with polarised infrared light perpendicular to the grating. (b) Energy band diagram of a metal contact to a p-type semiconductor and the 3-steps of the internal photoemission process: $p$ : photoexcitation, $t$ : transport, $e$ : emission. $E_{\mathrm{C}}$ and $E_{\mathrm{V}}$ are the conduction and valence band edges, respectively, $E_{\mathrm{F}}$ is the Fermi level, $E_{\mathrm{g}}$ is the energy bandgap of $\mathrm{Si}$, and $\Phi_{\mathrm{B}}$ is the Schottky barrier height. (c) Hot hole photoemission across the Schottky junction between a metal and a p-type semiconductor. 
The IPE mechanism is sketched in Figs. 1(b) and 1(c). IPE is a 3-step process consisting of the photoexcitation of hot (energetic) carriers in the metal, transport with scattering of hot carriers towards the metal-Si interface, and the emission of hot carriers over the Schottky barrier into the semiconductor where they are collected as the photocurrent. IPE requires that the energy of the incident photons, $h v$ ( $h$ is Plank's constant and $v$ is the optical frequency), be greater than the Schottky barrier energy, $\Phi_{\mathrm{B}}$. This mechanism is useful for detection at energies below the semiconductor bandgap, $E_{\mathrm{g}}$.

\section{Theoretical}

The grating coupler is highly suitable because arbitrary wavenumbers for diffracted light can be generated by simply changing the grating pitch. The grating coupling condition for SPPs on a metal surface is given by:

$$
k_{s p p}=k_{0} \sin \left(\theta_{i}\right)+M \frac{2 \pi}{\Lambda}
$$

where $k_{\text {spp }}$ is the wave number of the SPP propagating in the plane of the metal surface, $k_{0}$ is the wave number of the incident light, $\theta_{\mathrm{i}}$ is the angle of incidence of the light, $M$ is the grating order and $\Lambda$ is the grating pitch. We set to $\theta_{\mathrm{i}}=0^{\circ}$ for broadside excitation along the surface normal. Choosing $M=1$ simplifies Eq. (1) to the following:

$$
\Lambda=\frac{2 \pi}{k_{s p p}}=\frac{\lambda_{0}}{n_{e f f}}
$$

where $n_{\text {eff }}$ is the average effective refractive index of the SPP propagating along the structure including the grating. Equation (2) is used to obtain an initial value for $\Lambda$, using $n_{\text {eff }}$ for SPPs localised along the $\mathrm{Au} / \mathrm{Si}$ interface, from which the design of the grating is optimised via numerical modelling.

The substrate on which the devices are fabricated is a double-side polished wafer, consisting of a substrate $\left(\mathrm{p}^{+}-\mathrm{Si}\right) l_{2}=250 \pm 25 \mu \mathrm{m}$ thick, of carrier density $N_{\mathrm{A}}=5 \times 10^{18}$ to 3.5 $\times 10^{18} \mathrm{~cm}^{-3}$, bearing an epitaxial layer (p-Si) $l_{1}=3 \pm 0.5 \mu \mathrm{m}$ thick, of carrier density $N_{\mathrm{A}}=1.7$ $\times 10^{15}$ to $1.0 \times 10^{15} \mathrm{~cm}^{-3}$. We used this doping information to determine the refractive indices of these layers at $\lambda_{0}=1550 \mathrm{~nm}$ as 3.477 and 3.4735 for the p-Si and $\mathrm{p}^{+}$-Si layers, respectively [26]. Au is selected as the metal for the patch and the grating, and its refractive index was taken as $0.55+i 11.5$ [27].

The incident wave was set to a Gaussian beam at $\lambda_{0}=1550 \mathrm{~nm}$ and its polarization was transverse magnetic (TM) (i.e., electric field parallel to the $x$-direction). The finite difference time domain (FDTD) method [28], with a discretisation mesh size of $1 \mathrm{~nm} \times 0.5 \mathrm{~nm} \times 0.0001$ $\mathrm{nm}(x-y-z)$ around the grating, was used to model the optical performance of a cross-section (invariant along $y$ ) of several designs to determine good candidates for the device. Perfectly matched layers (PML) backed by perfect electric conductor (PEC) boundary conditions were used to terminate the computational domain in the $z$-direction along the top and bottom. The reflectance $\left(R_{0}\right)$, transmittance $\left(T_{0}\right)$, absorptance $\left(A_{0}\right)$ and coupling coefficient $\left(C_{0}\right)$ to SPPs are computed. The absorptance is calculated using:

$$
A_{0}=1-R_{0}-T_{0}
$$

The coupling coefficient is taken as the difference between transmittance spectra at the top of the Si substrate $(z=0)$ and the bottom of the grating (at $z=t)$ :

$$
C_{0}=T_{0}(z=t)-T_{0}(z=0)
$$

By adopting this definition we include the loss by direct absorption in Au within the coupling coefficient but the former is negligible compared to the latter for a thin Au film in the 
infrared. Through extensive modelling, good dimensions for the grating were found to be $W=$ $200 \mathrm{~nm}, H=80 \mathrm{~nm}$, and $t=20 \mathrm{~nm}$ for the metal patch. The spectral response of the structure was investigated by varying the duty cycle and the pitch for the cases of $\Lambda=390,400$ and $410 \mathrm{~nm}$. Simulations show that duty cycles of $60-70 \%$ produce the maximum absorptance, which is desirable for this photodetector design.

Figures 2(a)-2(c) show the computed $R_{0}, T_{0}, A_{0}$ and $C_{0}$ for photodetectors having $\Lambda=390$, 400 and $\Lambda=410 \mathrm{~nm}$ respectively. The subscript " $d$ " (dashed curves) represents the initial design whereas the subscript " $f$ " (solid curves) identifies the computed response of fabricated devices using their real dimensions. In general, the absorptance and coupling coefficient are largest at the wavelengths where Eq. (1) is satisfied, and the peak value red-shifts with $\Lambda$ as expected. The absorptance of the photodetector is mostly due to coupling of light to SPPs which are then absorbed as they propagate: in the case of, e.g., the design having $\Lambda=400 \mathrm{~nm}$ and $t=20 \mathrm{~nm}$ (Fig. 2(b)), on resonance $\left(\lambda_{0}=1560 \mathrm{~nm}\right)$ the peak value of $A_{0, \mathrm{~d}}$ is 0.42 and the peak value of $C_{0, \mathrm{~d}}$ is 0.38 , the difference being due to direct optical absorption of the incident Gaussian beam in the metal grating. It is worth pointing out that the metal loss is usually considered harmful in most optoelectronic devices but here this parameter plays an important role because its consequence is the generation of useful hot carriers in the metal.
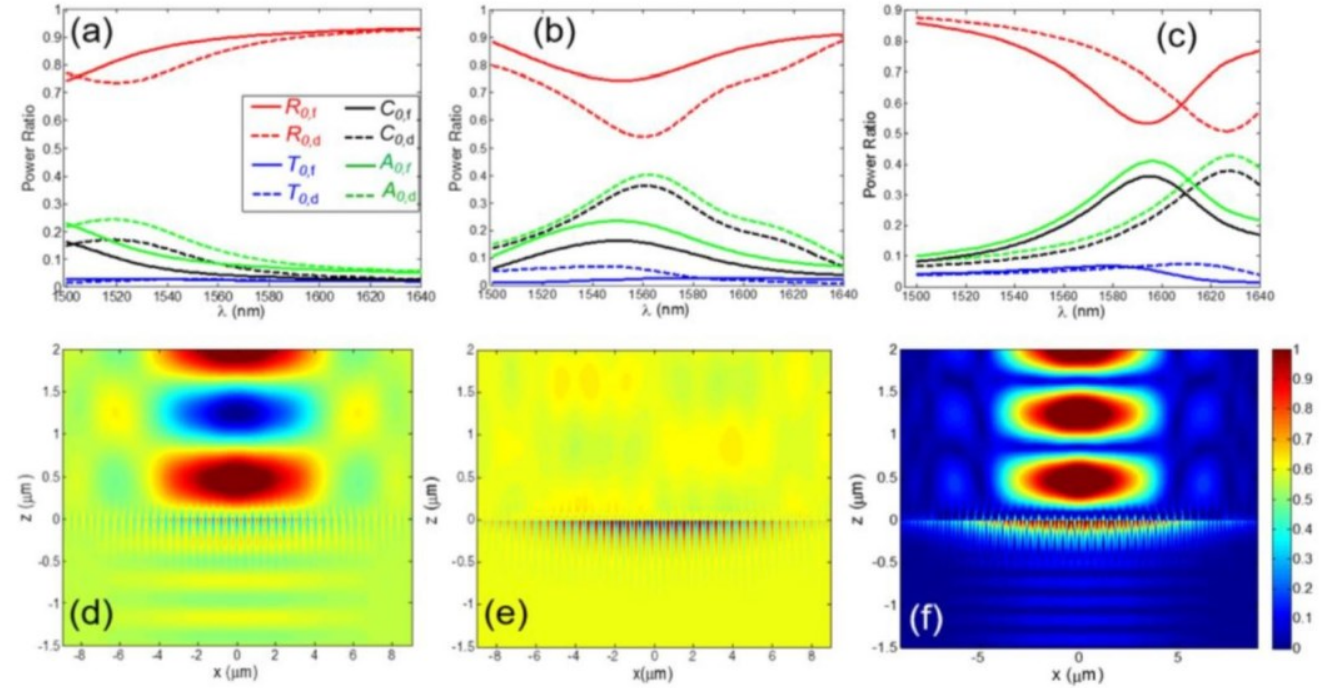

Fig. 2. Power ratios (absorptance $A_{0}$; transmittance $T_{0}$; reflectance $R_{0}$; and coupling coefficient $C_{0}$ ) of grating photodetectors operating near $1550 \mathrm{~nm}$, with a duty cycle of $60 \%$ and $t=20 \mathrm{~nm}$ (solid lines), and a duty cycle of $62 \%$ and $t=28.9 \mathrm{~nm}$ (dashed lines) for grating designs having $W=200 \mathrm{~nm}, H=80 \mathrm{~nm}$ and (a) $\Lambda=390 \mathrm{~nm}$, (b) $\Lambda=400 \mathrm{~nm}$, and (c) $\Lambda=410 \mathrm{~nm}$. The resonance red-shifts with increasing pitch. Electric field distribution of the gratings corresponding to $\Lambda=400 \mathrm{~nm}, t=20 \mathrm{~nm}$, computed on resonance $\left(\lambda_{0}=1560 \mathrm{~nm}\right)$; (d) $\operatorname{Re}\left\{E_{\mathrm{x}}\right\}$, (e) $\operatorname{Re}\left\{E_{z}\right\}$ and (f) $|\boldsymbol{E}|$ (also see Visualization 1 and Visualization 2). The incident electric field strength was $1 \mathrm{~V} / \mathrm{m}$.

Figures 2(d)-2(f) show the distribution of electric fields over the cross-section of the device for the case $\Lambda=400 \mathrm{~nm}, t=20 \mathrm{~nm}$, computed on resonance $\left(\lambda_{0} \sim 1560 \mathrm{~nm}\right)$. Figure 2(d) shows $\operatorname{Re}\left\{E_{\mathrm{x}}\right\}$, associated primarily with the incident Gaussian beam. Portions of the incident beam are absorbed, coupled to SPPs, reflected and transmitted through the grating. Figure 2(e) shows $\operatorname{Re}\left\{E_{\mathrm{z}}\right\}$ associated with SPPs propagating laterally (in the $\pm x$ directions) along the Au/p-Si interface, consistent with our sketch of Fig. 1(a). Figure 2(f) shows $|\boldsymbol{E}|$, summarising the contribution of all field components. A movie of $|\boldsymbol{E}|$, showing light propagation and scattering in the device is given in Visualization 1 and Visualization 2. It is observed from these field distributions that strong localised fields along the $\mathrm{Au} / \mathrm{p}-\mathrm{Si}$ interface 
are produced, which in turn will produce a considerable density of hot carriers in the metal along the Schottky contact as the SPPs are absorbed, ultimately enhancing IPE.

The detection wavelength can be tuned by varying the grating pitch $\Lambda$ (the resonance redshifts with increasing pitch). The photodetector can be optimized to operate at any infrared wavelength of sufficient photon energy to overcome $\Phi_{\mathrm{B}}$. (i.e.; from $\sim 1100$ to $4000 \mathrm{~nm}$ in this case). The full-width-at-half-maximum (FWHM) is another important parameter in the design of a resonant photodetector which is defined as the difference between the two wavelengths corresponding to the half-values of the peak of the absorptance curve, expressed in the frequency domain as:

$$
\Delta v=\frac{c_{0}}{\lambda_{0}^{2}} \Delta \lambda
$$

where $c_{0}$ is the speed of light in vacuum and $\lambda_{0}$ is the vacuum resonant wavelength. For our three pitches, using the absorptance spectra and this formula, we compute an optical FWHM bandwidth of $\sim 75 \mathrm{~nm}$ which is reasonably broadband.

The proposed photodetector is then analyzed by investigating its detection performance over a range of wavelengths. The photocurrent generation mechanism is IPE [29], wherein conduction carriers in the metal absorb energy quanta $h v$ from the propagating SPPs. Gaining enough energy, hot carriers can cross over the Schottky barrier into the Silicon where they are collected as photocurrent under reverse bias as long as $h v>\Phi_{\mathrm{B}}$. The internal quantum efficiency for a thick-film Schottky barrier, $\eta_{\mathrm{i}}$, which is dependent on the emission probability of hot carriers at the gold-silicon interface, is given by [30]:

$$
\eta_{i}=\frac{1}{2}\left(1-\sqrt{\frac{q \varphi_{B}}{h v}}\right)^{2}
$$

where $q$ is the charge of an electron. The responsivity, defined as the ratio of the photocurrent to the incident power, is:

$$
R_{e s p}=\kappa \frac{A q \eta_{i}}{h v}=\frac{I_{p h}}{P_{i n c}}
$$

where $A$ is the absorptance of the structure, $\kappa$ is the fraction of the absorptance that contributes to the photocurrent [31] (we assume $\kappa=1$ in this study), $I_{\mathrm{ph}}$ is the generated photocurrent and $P_{\text {inc }}$ is the incident power. The computed internal quantum efficiency and responsivity at $\lambda_{0}=1550 \mathrm{~nm}$, using $A_{0, \mathrm{~d}} \sim 0.4$ (Fig. 2), are $\eta_{\mathrm{i}} \sim 0.06$ and $R_{\text {esp }} \sim 30 \mathrm{~mA} / \mathrm{W}$.

\section{Fabrication and experimental results}

$\mathrm{Au}$ patches of diameter in the range of 4 to $30 \mu \mathrm{m}$ were fabricated on $\mathrm{p}-\mathrm{Si}$ on $\mathrm{p}^{+}-\mathrm{Si}$ wafers with the doping levels given above. The patches are circular to avoid field discontinuities and premature breakdown at corners, and each includes a contact arm with a probing pad. The patches were defined on the wafer's topside (i.e.; on the p-Si epitaxial layer) using a bi-layer liftoff lithography technique with a UV contact mask aligner to expose the resist. Immediately prior to metal patch deposition, the wafers were placed in a buffered hydrofluoric (HF) acid solution to remove any native $\mathrm{SiO}_{2}$ and allow intimate contact between the $\mathrm{Au}$ and the semiconductor. The grating duty cycles considered were $20 \%$ and $60 \%$ along with grating periods near $400 \mathrm{~nm}$ targeting telecom wavelengths $(\sim 1550 \mathrm{~nm})$. The layout of the Au gratings was drawn using e-LiNE software [32] and e-beam lithography was used to define the grating ridges in a bi-layer PMMA lift-off process. Additional details on the fabrication of the patches and gratings can be found in [33]. Sintered Al was used for the Ohmic contact on 
the backside. No cover layer was used to allow for direct electrical probing and photocurrent measurements.

Figures 3(a) and 3(b) show a scanning electron microscope (SEM) image and an amic force microscope (AFM) image of a fabricated grating photodetector. The metal thickness and roughness were deduced from AFM scans of the metal patch and grating ridges (e.g., Fig. $3(\mathrm{~b})$ ), revealing a thickness of $t=28.9 \mathrm{~nm}$ with a root mean square (RMS) roughness of $3 \mathrm{~nm}$ for the patch and $H=79 \mathrm{~nm}$ with an RMS roughness of $1 \mathrm{~nm}$ for the ridges. The width and spacing of the ridges are $W=252 \mathrm{~nm}$ and $154 \mathrm{~nm}$, yielding a pitch of $\Lambda=406 \mathrm{~nm}(400 \mathrm{~nm}$ designed) and a duty cycle of $62 \%$ (60\% designed). All dimensions are well in keeping with the target ones, except for the thickness of the patch $(28.9 \mathrm{~nm} \mathrm{vs}$. the target of $20 \mathrm{~nm})$. The low surface roughness on all metal surfaces (ridges and patch), attest to the good quality of the thermally evaporated Au.
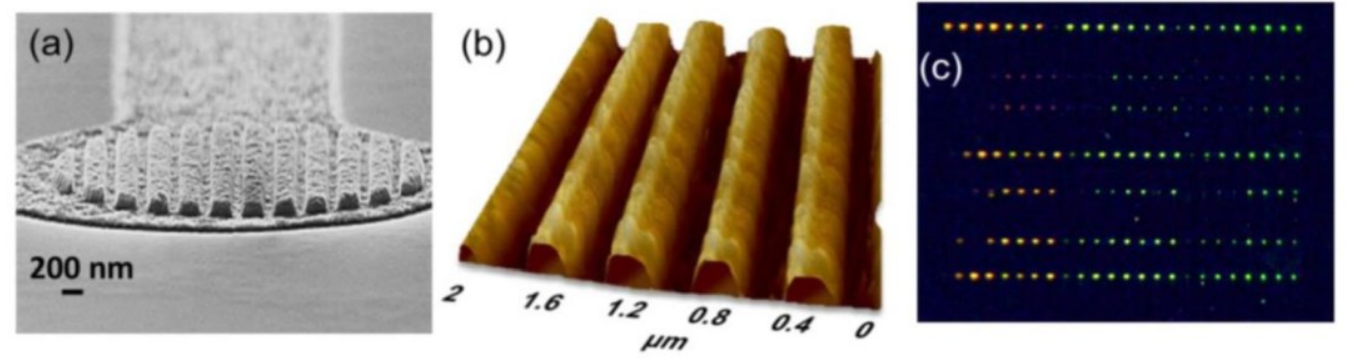

Fig. 3. (a), (b) SEM and AFM images, respectively, of an uncoated Au/p-Si grating photodetector. The pitch of the device is measured as $406 \mathrm{~nm}$ (400 nm designed) and the thickness of the Au patch is measured as $28.9 \mathrm{~nm}(20 \mathrm{~nm}$ designed). The duty cycle of this device is $60 \%$. (c) Optical microscope image of photodetectors of varying grating period, ebeam energy dose factors and duty cycles. Each color spot corresponds to one detector. The area shown is about $8 \mathrm{~mm}^{2}$ and contains $\sim 170$ detectors.

Figure 3(c) shows a microscope image of $\sim 170$ detectors distributed over a $\sim 8 \mathrm{~mm}^{2}$ area. The image was taken at a shallow angle in order to produce visible higher-order diffraction from each device. Each color spot corresponds to one detector, and the diffracted color changes with the period of the design. In each row, each yellow or orange spot corresponds to a detector with $\Lambda=390 \mathrm{~nm}$ but of e-beam energy dose factors of $1,1.1,1.2$ and 1.3 and different duty cycles of $20 \%$ and $60 \%$. Likewise, light green and dark green spots correspond to devices with $\Lambda=400 \mathrm{~nm}$ or $\Lambda=410 \mathrm{~nm}$, respectively.

A photodetector under test is biased by probing its probe pad and grounding its $\mathrm{Al}$ contact (Fig. 1(c)). A cleaved polarisation-maintaining single-mode fibre (PM-SMF) is used to excite the photodetector under test from the top. The fibre is manipulated using a six-axis micropositioner. A Photonetics TUNIC-Plus tunable external cavity laser is used to generate the incident light at $\lambda_{0} \sim 1550 \mathrm{~nm}$. The laser is thermally stabilized and linearly polarized, with the polarisation aligned along the slow axis of the PM-SMF. The setup is placed on a vibration isolation optical table and is shielded from background light. Instrument control and data acquisition are performed via computer using LabVIEW (SP1 2013) [34]. The optical power incident on a photodetector is determined by removing the losses of all the elements in the input path of the setup.

The experiments are conducted by placing a die under test under microscope and using tungsten probes attached to micropositioners to make electrical contact to a device via its probe pad. A reverse-bias voltage applied between the probe pad and the Al Ohmic contact on bottom surface (ground). Another micropositioner is then used to align the PM-SMF (polarisation-aligned to launch TM light) to the photodetector. The cleaved fibre output field behaves as a Gaussian beam, with the beam waist coincident with the fibre output facet. The fibre is aligned to the photodetector by placing the device under reverse-bias and maximising 
the measured photocurrent. When the fibre is rotated to produce TE-polarised incidence, almost no photocurrent is generated. Once the best possible alignment is achieved, a computer automated routine controlling ancillary instrumentation is run to rapidly execute test routines and gather measurement data. Ancillary instruments consisted of a laser tunable over $\sim 100 \mathrm{~nm}$ and a sourcemeter (Keithley 2400) used to apply a bias voltage and read the total current (dark + photo) in another channel simultaneously. All measurements were obtained at room temperature.

The red curve in Fig. 4(a) shows an average of five measured $I-V$ curves for a Schottky photodetector without illumination, comprising a Au patch of diameter of $28.9 \mu \mathrm{m}$ bearing a grating of pitch $\Lambda=400 \mathrm{~nm}$ designed to work at $\sim 1550 \mathrm{~nm}$. It shows the rectifying property of the Schottky structure. The reverse bias characteristics were rather soft for all devices, due to the low Schottky barrier and possibly to deep traps formed by the diffusion of $\mathrm{Au}$ into $\mathrm{p}-\mathrm{Si}$ during fabrication (Fig. 4(c)). The dark current density, although high in low-barrier structures in general, produced a manageable dark current given the small area of the contacts. The contact area of our test device (including the pad) is $A_{c}=767 \mu^{2}$. Isolation of the pad from the Si can be achieved by adding an intervening dielectric, which would result in a reduction of the dark current and an increase in the photodetector's signal to noise ratio.
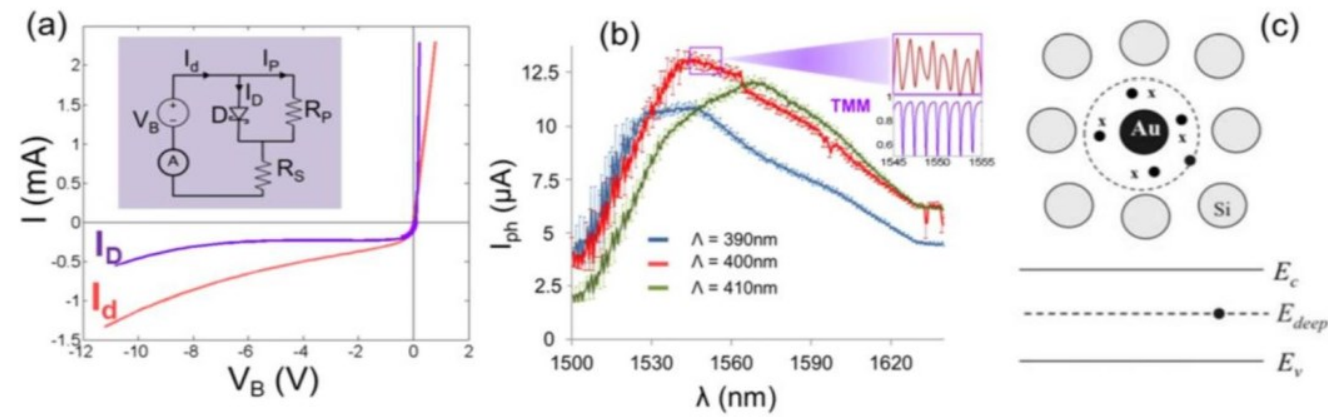

Fig. 4. (a) The red curve shows an average of five dark $I-V$ characteristics for a grating photodetector of diameter $28 \mu \mathrm{m}$ and $\Lambda=400 \mathrm{~nm}$. The purple curve is obtained after removing the effects of the shunt and series resistors shown in the non-ideal model in inset. (b) Measured photocurrent response of three grating photodetectors; $V_{\mathrm{B}}=-100 \mathrm{mV}, \Lambda=390,400$ and 410 $\mathrm{nm}$, duty cycle of $62 \%$ and patch diameter of $25 \mu \mathrm{m}$. A 4-period moving average is plotted on each response as the bold curve. The rapid wavelength variations correspond to Fabry-Perot resonances - the upper right inset is an enlarged response showing such resonances and the lower right inset shows a response calculated using the TMM method, (c) Deep traps formed by the diffusion of $\mathrm{Au}$ into $p-\mathrm{Si}$.

The dark current of a Schottky diode is given by:

$$
I_{D}=A_{c} A^{* * *} T^{2} e^{-\frac{q \varphi_{B}}{n k T}}
$$

where $k$ is Boltzmann's constant, $n$ is the ideality factor of diode, $T$ is the absolute temperature, $A^{* *}$ is the effective Richardson constant $\left(32 \mathrm{Acm}^{-2} \mathrm{~K}^{-2}\right.$ for holes [35]) and $A_{c}$ is the Schottky contact area. Solving Eq. (8) to obtain $\Phi_{\mathrm{B}}$ is not very accurate because the experimental setup and the photodetectors have a high resistance. The reverse bias and small forward bias regimes are affected by the shunt current $I_{P}$, and the shunt and series resistances, $R_{P}$ and $R_{S}$, shown in the equivalent circuit of a non-ideal diode in inset to Fig. 4(a). Several methods can be applied to extract the barrier height of high resistance Schottky diodes. The methods proposed by Lien et al. [36] and Werner [37] have been shown to yield accurate results. Following the first method [36] yields $R_{P}=17.11 \mathrm{k} \Omega, R_{S}=311 \Omega$ and $\Phi_{\mathrm{B}}=0.33 \mathrm{eV}$, whereas the second method [37] yields $R_{P}=16.88 \mathrm{k} \Omega, R_{S}=296 \Omega$ and $\Phi_{\mathrm{B}}=0.32 \mathrm{eV}$. Removing the effects of the resistor yields the purple $I-V$ curve for the diode shown in Fig. 
4(a). The ideality factor was found to be 2.13 and 2.08 , respectively. Given that $h v>\Phi_{\mathrm{B}}$ must be satisfied in order for the SPPs to be detected, the long-wavelength cut-off of the photodetectors is $\lambda_{0} \sim 3880 \mathrm{~nm}$ implying a broad range of operating wavelengths limited by the grating design.

Figure 4(b) plots the $p$-polarized photocurrent response measured at normal incidence with $P_{\text {inc }}=1.4 \mathrm{~mW}$ and $V_{\mathrm{B}}=-100 \mathrm{mV}$ for three grating detectors of different period. Two wavelength dependencies are observed on each response. The first one is a rapid variation which comes from Fabry-Perot resonances, as light transmitted through the grating and metal patch propagates through the semi-transparent substrate $\left(\mathrm{p}^{+}-\mathrm{Si}\right)$ and is reflected from the backside Ohmic contact. The right inset shows two plots, the upper is an enlarged plot of the measured photocurrent response near $1550 \mathrm{~nm}$ and the lower is the computed response of a three-layer structure comprising a $30 \mathrm{~nm}$ thick $\mathrm{Au}$ film, the $\mathrm{p}-\mathrm{Si}$ epi layer, and the $\mathrm{p}^{+}-\mathrm{Si}$ substrate bounded by air on top and $\mathrm{Al}$ on the bottom, obtained using the transfer matrix method (TMM) [38]. The measured rapid variations and the computed response are in good agreement, as the free spectral range (FSR) of the Fabry-Perot responses (given by the difference between the frequencies of two adjacent peaks) is in good agreement; FSR $\sim 1.87$ THz. The Fabry-Perot resonances would disappear if a more heavily doped Si substrate was used. The second wavelength dependency observed in the main panel of Fig. 4(b) is the slow one highlighted as the moving average plotted in bold on each grating response, and is due to the incident light coupling into SPPs. We measured the photoresponse of each device three times; they are all repeatable with a normalized mean square error (NMSE) on the photocurrent relative to the averaged photoresponse of $\sim 0.02-0.025 \mu \mathrm{A}$. These bold responses follow the absorptance responses labeled $A_{0, \mathrm{f}}$ in Figs. 2(a)-2(c), computed using the actual dimensions $(t=28.9 \mathrm{~nm}$, duty cycle of $62 \%)$. We calculated the photocurrent enhancement factor of the photodetector, relative to one without the grating but a $100 \mathrm{~nm}$ thick Au film, as $12.86 \mu \mathrm{A} / 2.48 \mu \mathrm{A}=5.18$.

The peak of the bold responses clearly red-shifts as the grating pitch increases from 390 to $410 \mathrm{~nm}$, following Eq. (1). Photocurrents were then measured as a function of incident optical power (emerging from the PM-SMF) at the resonance peak of each response, $\lambda_{01}=1537, \lambda_{02}=$ 1548 and $\lambda_{03}=1570 \mathrm{~nm}$ with $V_{\mathrm{B}}=-100 \mathrm{mV}$ applied. It should be noted that only the incident power overlapping with the device area couples to SPPs and contributes to the photocurrent and responsivity. As shown in the inset of Fig. 5(a), a portion of the incident power does not overlap with the device. There are two ways to refine the measurements to take this into account. One is to use a tapered fiber to focus the incident beam onto the photodetector area. The other is to correct the incident power assuming that a Gaussian beam emerges from the fibre, such that the beam diameter at the surface of the device can be estimated, and the fraction of overlapping incident power determined:

$$
\frac{P_{i n c, d}(s)}{P_{i n c}(0)}=1-e^{-\frac{2 s^{2}}{w_{s}^{2}}}
$$

where $s$ is the distance from the fiber tip to the device, $P_{\text {inc,d }}$ is the incident power that overlaps with the device area, $P_{\text {inc }}$ is the emerging beam power at the fiber tip, and $w_{\mathrm{s}}$ is the beam width at $s$ which is obtained from the beam waist, $w_{0}$, and $s$ via [39]:

$$
w_{s}=w_{0} \sqrt{1+\frac{s^{2}}{z_{0}^{2}}}
$$

$z_{0}$ is the Rayleigh range, computed directly from $w_{0}$ and the wavelength using $z_{0}=\pi w_{0}{ }^{2} / \lambda_{0}$. Knowing that the core diameter of our cleaved fiber is $2 w_{0}=8.2 \mu \mathrm{m}$, the cladding diameter is $125 \mu \mathrm{m}$, the angle between the tungsten probe intercepting the fiber and the device surface is $\theta \approx 45 \sim 50^{\circ}$, we find $s \approx 50 \sim 55 \mu \mathrm{m}$. Substituting this in Eqs. (10) and (9), we determine $P_{\text {inc,d }}$. 
Typical measurement results are shown in Fig. 5(a). The slope of the curves yields the responsivity, Eq. (7). We fit the measured data points to a linear model and take the slope of the fit as the responsivity of the photodetector, yielding $R_{\text {esp }}=11.82,12.95$ and $12.46 \mathrm{~mW} / \mathrm{A}$ for the photodetectors having $\Lambda=390,400$ and $410 \mathrm{~nm}$, respectively. These are among the highest responsivities measured at telecom wavelengths $(\sim 1550 \mathrm{~nm})$ for sub-bandgap detectors on $\mathrm{Si}$.

Figure 5(b) plots the responsivity as a function of reverse bias, extracted from the measurements of Fig. 5(a) and from additional measurements at other bias points, revealing that the responsivity increases significantly with bias. Applying a reverse bias to a Schottky photodetector reduces $\Phi_{\mathrm{B}}$ via the Schottky effect, so the responsivity is expected to increase with reverse bias [40]. A reverse bias reduces the Schottky barrier $\Phi_{B}$ by an amount $\Delta \Phi_{B}$ via the Schottky effect [27]:

$$
\Delta \Phi_{B}=\left[\frac{q^{3} N\left(V_{R}+V_{b i}-k T / q\right)}{8 \pi^{2} \varepsilon_{S i}^{3}}\right]^{0.25}
$$

where $\Delta \Phi_{B}$ is in $\mathrm{eV}, q$ is the electronic charge, $N$ is the density of ionized impurities, $V_{\mathrm{R}}$ is the reverse bias voltage, $V_{\mathrm{bi}}$ is the built-in potential, $k$ is Boltzmann's constant, $T$ is the absolute temperature $(T=300 \mathrm{~K})$ and $\varepsilon_{\mathrm{Si}}$ is the permittivity of $\mathrm{Si}$ (taken as the static permittivity). Equations (6), (7) and (11) indicate that the responsivity will increase with reverse bias due to the Schottky effect, but only modestly.

A trend curve for the responsivity was computed using Eqs. (6), (7) and (11) and plotted in Fig. 5 (b) (we added an offset to the trend curve such that it would coincide with the measurement at low-bias in order to illustrate the effect of $\Delta \Phi_{\mathrm{B}}$ on the responsivity). For the computations, the Schottky barrier height and the built-in voltage were taken as $\Phi_{\mathrm{B}}=0.32 \mathrm{eV}$ and $V_{\mathrm{bi}}=0.26 \mathrm{~V}$, as extracted from the $I-V$ curve in Fig. 4(a), and the Fowler characteristics measured on a similar detector [11]. The change in Schottky barrier height over the range of reverse bias voltage considered does not explain the change in the measured responsivity. The large increase observed in Fig. 5(b) is more likely caused by deep traps in the Si bandgap, formed by Au migration into the Si during fabrication, which are uncovered with increasing reverse bias.

In perfect semiconductors, there exists a bandgap between the valence and conduction bands of energy $E_{\mathrm{g}}=E_{\mathrm{C}}-E_{\mathrm{V}}$ (Fig. 1(b)). During fabrication Au ions can migrate into the $\mathrm{Si}$ (driven by process temperatures) where they introduce energy states somewhere in the band gap, usually close to mid-gap $\left(\sim E_{\mathrm{g}} / 2\right)$, and thus are termed deep traps (Fig. 4(c)). They generally act as recombination-generation centres and are not classified as donor or acceptor levels. The reason is that the generation-recombination centres have to capture/emit both electrons and holes. The capture/emission cross-sections of a deep trap will depend upon several factors such as whether the process is capture or emission, the ionisation energy of the trap, whether the trap is neutral, or positively or negatively charged, and whether the trap is in the bulk or at the interface. A deep trap (e.g. Au in $\mathrm{Si}$ ) is most effective as a recombination centre if the hole capture time (from the valence band) is nearly the same as the electron capture time (from the conduction band). The trap levels for $\mathrm{Au}$ in $\mathrm{Si}$ are $0.54 \mathrm{eV}$ for acceptors and $0.29 \mathrm{eV}$ for donors [27], both of which are below the photon energy of the incident light, so traps can assist in producing photocurrent by acting as a mid-gap "bridge" for EHPs between the valence and conduction bands [41]. In Fig. 5(b), we suspect that such traps become increasingly uncovered as the reverse bias increases, thus enabling this photodetection channel resulting in increased responsivity. 


\section{Optics EXPRESS}
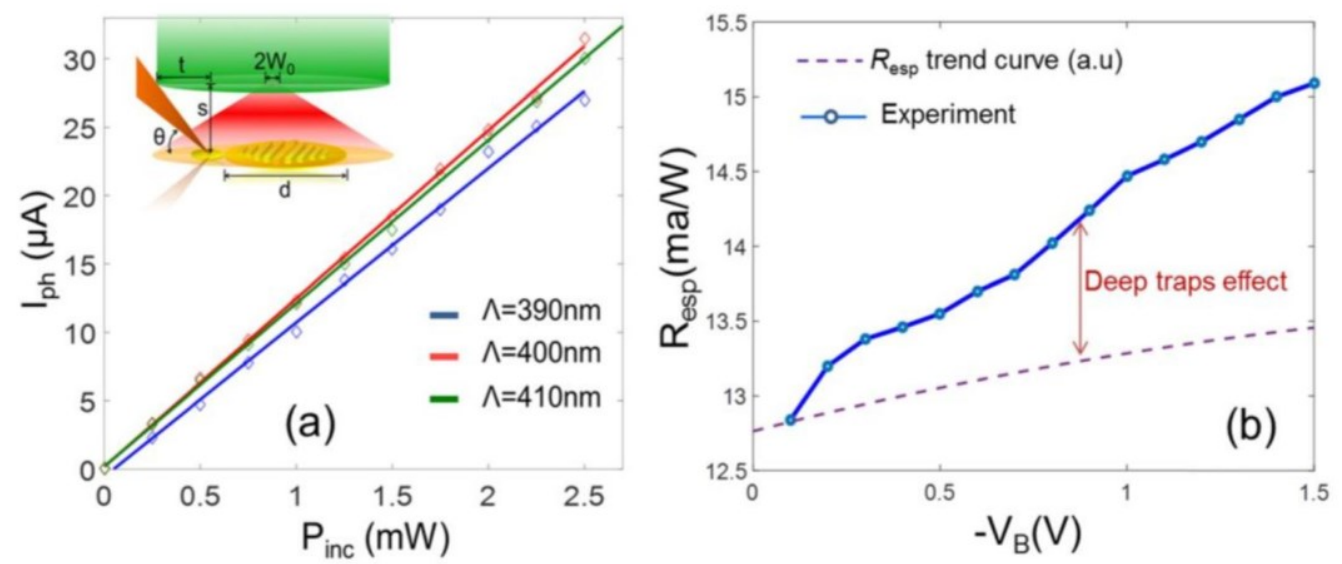

Fig. 5. (a) Photocurrent generated by grating detectors $v s$. incident power $P_{\text {inc,d }}$ measured for three different pitches: $\Lambda=390 \mathrm{~nm}\left(\lambda_{01}=1537 \mathrm{~nm}\right), \Lambda=400 \mathrm{~nm}\left(\lambda_{02}=1548 \mathrm{~nm}\right)$ and $\Lambda=410$ $\mathrm{nm}\left(\lambda_{03}=1570 \mathrm{~nm}\right)$. A linear fit is applied to the data and the slope corresponds to the responsivity of the device. (b) Responsivity vs. reverse bias measured for the photodetector with $\Lambda=400 \mathrm{~nm}$ at $\lambda_{0}=1548 \mathrm{~nm}$; the dashed curve shows a trend curve for the responsivity obtained by taking into account the Schottky barrier lowering effect.

\section{Conclusion}

In summary, we have presented a broadband silicon plasmonic Schottky photodetector with a high responsivity operating in the sub-bandgap regime exploiting internal photoemission. A metal grating is used to couple perpendicularly-incident light to SPPs propagating along a thin metal patch forming a Schottky contact to p-Si. The responsivity is enhanced by the absorption of SPPs directly along the Schottky contact leading to the creation of hot holes near the contact. These holes cross over the Schottky barrier $(\sim 0.32 \mathrm{eV})$ and are collected as a photocurrent. Our measurements reveal a high responsivity of $\sim 13 \mathrm{~mA} / \mathrm{W}$ at telecom wavelengths $(\sim 1550 \mathrm{~nm})$ and under low reverse bias. The responsivity increases with reverse bias. The optical bandwidth (FWHM) is $\sim 80 \mathrm{~nm}$. The dark current performance of the photodetector could be significantly improved by using a smaller area and fabricating the contact arm and probing pad on a higher level (e.g., above a dielectric). These detectors have many important advantages such as speed, simplicity, compatibility with silicon, and low-cost fabrication.

\section{Acknowledgments}

The authors are grateful to Chengkun Chen for assistance with the modelling. Financial support from the Natural Sciences and Engineering Research Council of Canada and from Test Photonics Canada is gratefully acknowledged. 


\section{Chapter: Design a High-Sensitivity Schottky-Contact Plasmonic}

\section{Nanoantenna}

The following article entitled "Schottky-contact plasmonic dipole rectenna concept for biosensing" was published in Optics Express, Volume 24, Issue 20 on Feb. 2013 and is presented in full. The article represents work performed solely by the primary author under the supervision and guidance of the co-authors.

In this manuscript, we present an array of metal nano-dipoles supporting short-range surface plasmon polaritons (SPPs) integrated into a silicon-based Schottky-contact photodetector. Photodetection occurs via the internal photoelectric effect for photon energies above the Schottky barrier height and below the bandgap of Si. Excitation through the substrate by a normally-incident plane wave polarized along the length of the dipoles excites a specific strongly-confined SPP mode on the antennas ( $s a_{b}^{0}$ [P. Berini, Phys. Rev. B 63, 125417 (2001)]). Calculations of the spectral response of the device predict a high responsivity of $100 \mathrm{~mA} / \mathrm{W}$ and practical minimum detectable powers of $-12 \mathrm{dBm}$ in the infra-red $(1310 \mathrm{~nm})$. The proposed device was also investigated for use as a biosensor by computing its bulk and surface sensitivities. Sensitivities of $\sim 250 \mathrm{~nm} / \mathrm{RIU}$ (bulk) and $\sim 8 \mathrm{~nm} / \mathrm{nm}$ (surface) in water are predicted. In terms of changes in photocurrent, an incident optical power of $1 \mathrm{~mW}$ would yield a change of $\sim 1 \mu \mathrm{A}$ as an adlayer grows to $5 \mathrm{~nm}$ in thickness, which is readily measurable. We also apply a transmission line model to describe the performance of the antennas, and we extract two useful formulas to predict their bulk and surface sensitivities. We prove analytically that the sensitivities of dipoles are much greater than those of similar 
monopoles and they show that this difference comes from the gap in dipole antennas where electric fields are strongly enhanced.

Correction in Fig.3: Labels A and R must be swapped. The curves, data and colors assigned all are right, but there is a mislabeling in the axes. The corrected figure is the one that $\mathrm{A}$ is at the left axis and instead $\mathrm{R}$ is at the right axis.

Correction in equation (9): $f(x, y)$ must change to $f(y, z)$

Discussion on equations (7) to (9): we calculated the characteristic impedance of transmission line model using this definition: $Z_{0}=P / I^{2}$, however it may happen that someone uses another definition such as $Z_{0}=V^{2} / P$ and get different characteristic impedance value. Final value for $Z_{0}$ should be approximately same using both methods. 


\title{
Schottky-contact plasmonic dipole rectenna concept for biosensing
}

\author{
Mohammad Alavirad, ${ }^{1}$ Saba Siadat Mousavi, ${ }^{2}$ Langis Roy, ${ }^{1}$ and Pierre \\ Berini $^{2,3}$,* \\ ${ }^{1}$ Department of Electronics, Carleton University, 1125 Colonel By Dr., \\ Ottawa, ON, K1S 5B6, Canada \\ ${ }^{2}$ School of Electrical Engineering and Computer Science, University of Ottawa, 800 King \\ Edward Ave., \\ Ottawa, ON, KIN 6N5, Canada \\ ${ }^{3}$ Department of Physics, University of Ottawa, 150 Louis Pasteur, \\ Ottawa ON, KIN 6N5, Canada \\ "berini@eecs.uottawa.ca
}

\begin{abstract}
Nanoantennas are key optical components for several applications including photodetection and biosensing. Here we present an array of metal nano-dipoles supporting surface plasmon polaritons (SPPs) integrated into a silicon-based Schottky-contact photodetector. Incident photons coupled to the array excite SPPs on the Au nanowires of the antennas which decay by creating "hot" carriers in the metal. The hot carriers may then be injected over the potential barrier at the Au-Si interface resulting in a photocurrent. High responsivities of $100 \mathrm{~mA} / \mathrm{W}$ and practical minimum detectable powers of $-12 \mathrm{dBm}$ should be achievable in the infra-red (1310 $\mathrm{nm})$. The device was then investigated for use as a biosensor by computing its bulk and surface sensitivities. Sensitivities of $\sim 250 \mathrm{~nm} / \mathrm{RIU}$ (bulk) and $\sim 8 \mathrm{~nm} / \mathrm{nm}$ (surface) in water are predicted. We identify the mode propagating and resonating along the nanowires of the antennas, we apply a transmission line model to describe the performance of the antennas, and we extract two useful formulas to predict their bulk and surface sensitivities. We prove that the sensitivities of dipoles are much greater than those of similar monopoles and we show that this difference comes from the gap in dipole antennas where electric fields are strongly enhanced.
\end{abstract}

(C) 2013 Optical Society of America

OCIS codes: (240.6680) Surface plasmons; (290.5850) Scattering particles; (310.6628) Subwavelength structures, nanostructures; (130.6010) Sensors; (240.0240) Optics at surfaces.

\section{References and links}

1. S. A. Maier, Plasmonics: Fundamentals and Applications (Springer, 2007), 1st ed.

2. B. Hecht, H. Bielefeldt, L. Novotny, Y. Inouye, and D. W. Pohl, "Local excitation, scattering, and interference of surface plasmons," Phys. Rev. Lett. 77, 1889-1892 (1996).

3. W. L. Barnes, T. W. Preist, S. C. Kitson, and J. R. Sambles, "Physical origin of photonic energy gaps in the propagation of surface plasmons on gratings," Phys. Rev. B 54, 6227-6244 (1996).

4. S. S. Mousavi, P. Berini, and D. McNamara, "Periodic plasmonic nanoantennas in a piecewise homogeneous background," Opt. Express 20, 18044-18065 (2012).

5. P. Berini, "Bulk and surface sensitivities of surface plasmon waveguides," New Journal of Physics 10, 105010 (2008).

\#181367 - \$15.00 USD Received 10 Dec 2012; revised 17 Jan 2013; accepted 18 Jan 2013; published 12 Feb 2013 
6. F. J. Rodriguez-Fortuno, M. Martinez-Marco, B. Tomas-Navarro, R. Ortuno, J. Marti, A. Martinez, and P. J. Rodriguez-Canto, "Highly-sensitive chemical detection in the infrared regime using plasmonic gold nanocrosses," Applied Physics Letters 98, 133118 (2011).

7. C.-Y. Tsai, S.-P. Lu, J.-W. Lin, and P.-T. Lee, "High sensitivity plasmonic index sensor using slablike gold nanoring arrays," Applied Physics Letters 98, 153108 (2011).

8. S. Lal, S. Link, and N. J. Halas, "Nano-optics from sensing to waveguiding," Nat Photon 1, $641-648$ (2007).

9. P. Berini, "Plasmon-polariton waves guided by thin lossy metal films of finite width: Bound modes of symmetric structures," Phys. Rev. B 61, 10484-10503 (2000).

10. E. M. Larsson, J. Alegret, M. Kll, and D. S. Sutherland, "Sensing characteristics of nir localized surface plasmon resonances in gold nanorings for application as ultrasensitive biosensors," Nano Letters 7, 1256-1263 (2007).

11. G. I. Stegeman, J. J. Burke, and D. G. Hall, "Nonlinear optics of long range surface plasmons," Applied Physics Letters 41, 906-908 (1982).

12. C. L. Nehl, H. Liao, and J. H. Hafner, "Optical properties of star-shaped gold nanoparticles," Nano Letters 6 , 683-688 (2006).

13. M. Piliarik, P. Kvasnička, N. Galler, J. R. Krenn, and J. Homola, "Local refractive index sensitivity of plasmonic nanoparticles," Opt. Express 19,9213-9220 (2011).

14. M. W. Knight, H. Sobhani, P. Nordlander, and N. J. Halas, "Photodetection with active optical antennas," Science 332, 702-704 (2011).

15. H. Wang, D. W. Brandl, F. Le, P. Nordlander, and N. J. Halas, "Nanorice: a hybrid plasmonic nanostructure," Nano Letters 6, 827-832 (2006). PMID: 16608292.

16. M. M. Miller and A. A. Lazarides, "Sensitivity of metal nanoparticle surface plasmon resonance to the dielectric environment," The Journal of Physical Chemistry B 109, 21556-21565 (2005).

17. F. Mazzotta, G. Wang, C. Hgglund, F. Hk, and M. P. Jonsson, "Nanoplasmonic biosensing with on-chip electrical detection," Biosensors and Bioelectronics 26, 1131 - 1136 (2010).

18. L. Guyot, A.-P. Blanchard-Dionne, S. Patskovsky, and M. Meunier, "Integrated silicon-based nanoplasmonic sensor," Opt. Express 19,9962-9967 (2011).

19. C. Wu, A. B. Khanikaev, R. Adato, N. Arju, A. A. Yanik, H. Altug, and G. Shvets, "Fano-resonant asymmetric metamaterials for ultrasensitive spectroscopy and identification of molecular monolayers," Nat Mater 11, 69-75 (2012).

20. M. Casalino, G. Coppola, M. Iodice, I. Rendina, and L. Sirleto, "Critically coupled silicon fabry-perot photodetectors based on the internal photoemission effect at $1550 \mathrm{~nm}$," Opt. Express 20, 12599-12609 (2012).

21. S. R. J. Brueck, V. Diadiuk, T. Jones, and W. Lenth, "Enhanced quantum efficiency internal photoemission detectors by grating coupling to surface plasma waves," Applied Physics Letters 46, 915-917 (1985).

22. C. Daboo, M. Baird, H. H. N. Apsley, and M. Emeny, "Improved surface plasmon enhanced photodetection at an augaas schottky junction using a novel molecular beam epitaxy grown otto coupling structure," Thin Solid Films 201, 9 - 27 (1991).

23. A. Akbari, R. N. Tait, and P. Berini, "Surface plasmon waveguide schottky detector," Opt. Express 18, 8505-8514 (2010).

24. S. Zhu, G. Q. Lo, and D. L. Kwong, "Theoretical investigation of silicide schottky barrier detector integrated in horizontal metal-insulator-silicon-insulator-metal nanoplasmonic slot waveguide," Opt. Express 19, 1584315854 (2011)

25. I. Goykhman, B. Desiatov, J. Khurgin, J. Shappir, and U. Levy, "Locally oxidized silicon surface-plasmon schottky detector for telecom regime," Nano Letters 11, 2219-2224 (2011).

26. E. S. Barnard, R. A. Pala, and M. L. Brongersma, "Photocurrent mapping of near-field optical antenna resonances," Nat Nano 6, 588-593 (2011).

27. J. McSpadden, L. Fan, and K. Chang, "Design and experiments of a high-conversion-efficiency 5.8-ghz rectenna," Microwave Theory and Techniques, IEEE Transactions on 46, 2053 -2060 (1998).

28. FDTD Solutions (Lumerical Solutions Inc.).

29. E. D. Palik, Handbook of Optical Constants of Solids (Academic Press, 1985).

30. D. J. Segelstein, "The complex refractive index of water," Master's thesis, University of Missouri, Kansas City, Missouri, USA (1981).

31. R. Soref and B. Bennett, "Electrooptical effects in silicon," Quantum Electronics, IEEE Journal of 23, 123 - 129 (1987).

32. P. Nordlander, C. Oubre, E. Prodan, K. Li, and M. I. Stockman, "Plasmon hybridization in nanoparticle dimers," Nano Letters 4, 899-903 (2004).

33. C. Scales and P. Berini, "Thin-film schottky barrier photodetector models," Quantum Electronics, IEEE Journal of 46, 633-643 (2010).

34. R. N. Stuart, F. Wooten, and W. E. Spicer, "Mean free path of hot electrons and holes in metals," Phys. Rev. Lett. 10, 119-119 (1963).

35. S. M. Sze and K. K. Ng, Physics of Semiconductor Devices (Wiley, 2006), 3rd ed.

36. A. Akbari, A. Olivieri, and P. Berini, "Sub-bandgap asymmetric surface plasmon waveguide schottky detectors on silicon," Accepted for publication in Sel. Top. Quantum Electronics, IEEE Journal of (2013). 
37. S. J. Zalyubovskiy, M. Bogdanova, A. Deinega, Y. Lozovik, A. D. Pris, K. H. An, W. P. Hall, and R. A. Potyrailo, "Theoretical limit of localized surface plasmon resonance sensitivity to local refractive index change and its comparison to conventional surface plasmon resonance sensor," J. Opt. Soc. Am. A 29, 994-1002 (2012).

38. J. Homola, "Surface plasmon resonance sensors for detection of chemical and biological species," Chemical Reviews 108, 462-493 (2008). PMID: 18229953.

39. V. Brioude and O. Parriaux, "Normalised analysis for the design of evanescent-wave sensors and its use for tolerance evaluation," Optical and Quantum Electronics 32, 899-908 (2000).

40. L. Tang, S. E. Kocabas, S. Latif, A. K. Okyay, D.-S. Ly-Gagnon, K. C. Saraswat, and D. A. B. Miller, "Nanometre-scale germanium photodetector enhanced by a near-infrared dipole antenna," Nat Photon 2, 226-229 (2008).

41. R. F. Harrington, Time-Harmonic Electromagnetic Fields (McGraw-Hill, 1961), 1st ed.

\section{Introduction}

Surface plasmon polaritons (SPPs) are of great interest in a wide range of fields from physics, chemistry, materials processing, to biology. SPPs are electromagnetic surface waves coupled to free electron oscillations propagating along a metal-dielectric interface at optical wavelengths [1]. SPPs have many interesting properties such as highly-enhanced fields [2], strong confinement (to sub-wavelength scale) [3,4] and high bulk and surface sensitivities [5-7]. These characteristics are of interest to several applications such as waveguides $[5,8,9]$, sensors [10], and nonlinear optics [11].

Advances in nanofabrication have made periodic metallic nanostructures and nanoantennas attractive for practical uses $[2,8,12]$. SPP biosensors have become key tools for the investigation of biomolecular interactions and for detection applications. Piliarik emphet al. measured the localized surface plasmon resonance shift of an arrays of chains of metallic nanorods in response to bulk refractive index changes as $140 \mathrm{~nm} / \mathrm{RIU}$ [13]. Knight et al. reported an active optical monopole array which can be considered as a highly compact, wavelength-specific, and polarization-specific light detector appropriate for the sensing applications [14]. Rodriguez et $a l$. reported a chemosensor fabricated from gold nano-crosses exhibiting a strongly localized SPP in the infrared and obtained a high bulk sensitivity of $740 \mathrm{~nm} / \mathrm{RIU}$ [6]. Tsai emphet al. described an optical sensor consisting of square-lattice slab-like gold nano-rings, reporting a bulk sensitivity of $691 \mathrm{~nm} / \mathrm{RIU}$ [7]. Star-shaped nanoparticles suitable for microscopic imaging exhibited a wavelength peak shift of $665 \mathrm{~nm} / \mathrm{RIU}$ [12]. Rice-shaped nanoparticles are reported to have even larger sensitivity but with a broader spectral response [15]. The bulk sensitivity of a rod-like metal nanoparticle surface plasmon resonance is reported in [16]; the natural resonance frequency of this structure strongly depends on its aspect ratio and thickness: narrow rods with a high aspect ratio have lower resonant frequencies. Bulk sensitivities reported for cylindrical rods of aspect ratio ranging from 0.5 to 3 are from 150 to $450 \mathrm{~nm} / \mathrm{RIU}$. Mazzotta et al. measured photocurrent ratio sensitivity of a nanoplasmonic biosensor chip with integrated electrical detection (an array of gold nanodisks) using hole-mask colloidal lithography and they achieved a value around $133 \mathrm{~nm} / \mathrm{RIU}$ as of it biosensing property [17]. Guyot et al. developed miniaturized silicon based nanoplasmonic biosensing platform as a symmetric nanohole array structure and got the sensitivity of $4 \times 10^{-5}$ RIU which is greatly improved and promising for the application in portable nanoplasmonic multisensing and imaging [18]. The bulk sensitivity was also calculated by a quasistatic theory which serves as an upper bound to sensitivities of nanoparticles on dielectric substrates such as those associated with biomolecule sensing. Achieving a sharp resonant peak (which is easier to track) is a difficult challenge with most plasmonic nanostructures. A multipixel array of Fano-resonant asymmetric metamaterial is presented in [19], which is capable of confining light to nanoscale regions with a very sharp resonant peak. The narrower linewidth of this structure can also increase the electric field enhancement in regions of the metamaterial, which improves the sensitivity.

A Schottky barrier photodetector formed at the interface between a metal and a lightly

\#181367 - \$15.00 USD Received 10 Dec 2012; revised 17 Jan 2013; accepted 18 Jan 2013; published 12 Feb 2013

(C) 2013 OSA

25 February 2013 / Vol. 21, No. 4 / OPTICS EXPRESS 4330 
doped semiconductor can be used to detect infrared radiation below the bandgap energy of the substrate via the internal photoelectric effect (IPE) [20]. Such detectors involving SPP excitations in grating [21], prism [22], waveguide [23-25] and antenna [14,26] geometries have been reported. The combination of a classical (microwave) antenna with a rectifier, termed a "rectenna", is characterized (in part) by its ability to convert microwave to de power [27]. In this paper we propose a plasmonic rectenna array comprised of Au nanowires of rectangular cross-section operating as optical dipole antennas. The difference between dipole and monopole antennas is the existence of a gap in the former where highly localised fields are existed. The dipoles are inter-connected electrically (in an optically non-invasive manner), on a $p$-doped $\mathrm{Si}$ substrate forming a Schottky contact thereon. The antennas are assumed covered with $\mathrm{H}_{2} \mathrm{O}$ to represent use as a biochemical sensor. We investigate the spectral response of the rectenna as a function of the index of the superstrate and with various thicknesses of biochemical adlayer grown on the antennas. We combine numerical methods (FDTD, modal analysis) with an analytical model for IPE, and a transmission line model for the nano-dipole antennas to determine the performance characteristics of the proposed structure.

First the rectenna geometry and the methods applied in its analysis are discussed. Secondly, a particular geometry is considered and computed results are presented. Thirdly, we discuss the operation of the rectenna as a Schottky contact detector and we estimate its responsivity and detection limit. Finally, we assess the potential of the structure for bulk and surface (bio)chemical sensing.

\section{Geometry}

Figure 1(a) shows our rectenna concept, consisting of an array of Au nano-dipole antennas on $\mathrm{Si}$, electrically interconnected via Au lines running perpendicularly to the dipole axes through the middle of each arm to a common circular contact pad; as the array is illuminated via $x$ polarized light, such interconnects do not affect the optical behavior of the array. The array is symmetric about the $x$ and $y$ axes. It is illuminated by a Gaussian beam focused onto the array through the substrate near $\lambda_{0}=1310 \mathrm{~nm}$ ( $\mathrm{Si}$ is transparent at this wavelength) and its response monitored via the photocurrent generated by (IPE) using the Au contact pad (on top) and the $\mathrm{Al}$ Ohmic contacts (below). In a biosensing application, this arrangement is advantageous as it simplifies interrogation, because only the photocurrent need be monitored, and it separates the optics (bottom) from the micro-fluidics (top). A single dipole is depicted in Fig. 1(b) showing our definition of its dimensions and the coordinate system used for the analysis; $a=0$ throughout except for the surface sensitivity computations.

The finite difference time domain (FDTD) method [28] with a $0.5 \times 0.5 \times 0.5 \mathrm{~nm}^{3}$ discretisation in the region around the antenna was used for the modeling along with Paliks material data [29] for $\mathrm{Au}$ and $\mathrm{Si}$, and Segelsteins data [30] for $\mathrm{H}_{2} \mathrm{O}$ which takes the water absorption into consideration. Transmittance and reflectance monitors were placed $2.5 \mu \mathrm{m}$ above and below the $\mathrm{Si} / \mathrm{H}_{2} \mathrm{O}$ interface, respectively, parallel to the $x-y$ plane. We consider the case of an array that is small compared to the waist of the Gaussian beam, so the beam can be considered as a plane wave in the simulations. Thus, an $x$-polarised plane wave source located $2.8 \mu \mathrm{m}$ below the interface illuminates the array along the $+z$ direction. We assumed doping levels for $p^{+}$-Si and $p$-Si of $10^{+18} \mathrm{~cm}^{-3}$ and $10^{+15} \mathrm{~cm}^{-3}$; the attenuation of a plane wave at $\lambda_{0}=1310 \mathrm{~nm}$ propagating through a $500 \mu \mathrm{m}$ thick $p^{+}$-Si substrate of this doping level, calculated using the Drude model [31], is $0.2037 \mathrm{~dB}$, which is negligible in this work.

\section{Optical response}

From Fig. 1(b), we define the nanoantenna length $(l)$, width $(w)$, thickness $(t)$, and the gap length $(g)$, as well as the vertical and horizontal distance $(p, q)$ between any two adjacent

\#181367 - \$15.00 USD Received 10 Dec 2012; revised 17 Jan 2013; accepted 18 Jan 2013; published 12 Feb 2013 (C) 2013 OSA 

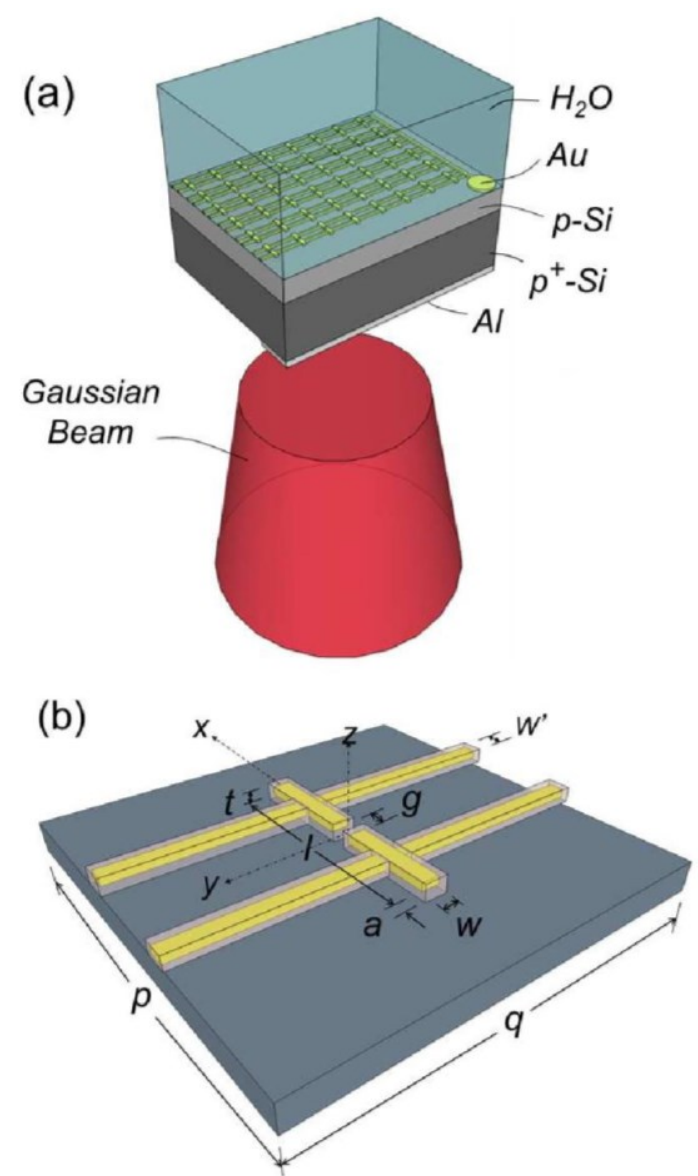

Fig. 1. (a) Array of $\mathrm{Au}$ dipoles on $p$-Si on $p^{+}-\mathrm{Si}$ covered by $\mathrm{H}_{2} \mathrm{O}$. $\mathrm{Al}$ Ohmic contacts are located at the bottom of the structure and an Au circular contact pad is connected to all dipole arms via optically non-invasive perpendicular Au interconnects. A plane wave source illuminates the array in the $+z$-direction from below. (b) Geometry of a unit cell of the array under study (interconnects are shown as well); the dipole is assumed covered by an adlayer of thickness $a$ when the surface sensitivities are computed.

nanoantennas. Also the interconnect width is labeled as $w^{\prime}$. Good dipole (infinitely periodic, "good dipole" means a dipole that resonates near a desired wavelength and that performs well in terms of enhancement and absorptance) dimensions were determined via modeling to be: $w=30 \mathrm{~nm}, t=30 \mathrm{~nm}, l=210 \mathrm{~nm}, g=20 \mathrm{~nm}$ and $p=q=300 \mathrm{~nm}$. Interconnect dimensions were taken as $w^{\prime}=20 \mathrm{~nm}$ and $t=30 \mathrm{~nm}$. All dimensions except for $g$ remain constant throughout the paper. Figure 2(a)-2(c) show the electric field distribution of the dipole array at resonance over $x-y$ cross-sections slightly inside the Au close to the $\mathrm{Si} / \mathrm{Au}$ interface, through the middle of the Au dipoles, and slightly above the Au surface in $\mathrm{H}_{2} \mathrm{O}$. As noted, the field intensity is very high in the dipoles near the Au/Si interface (Fig. 2(a)) and in the gap (Fig. 2(b)), compared to along the dipole arms and the ends. Localized fields in the gap make small-gap antennas highly sensitive to changes in this region (as discussed below), and strong fields in the Au near the $\mathrm{Au} / \mathrm{Si}$ interface are desirable to enhance IPE; both attributes are useful for the envisaged biosensors. It is evident that the Au interconnects have a negligible influence on the field distribution, and that the periodicity is large enough for coupling between two neighboring dipoles 
to be negligible. Given the uniform illumination and their length, the dipoles resonate in their lowest order bonding mode [32], in the $s a_{b}^{0}$ mode propagating along the nanowire waveguides forming the dipole arms $[4,5]$.

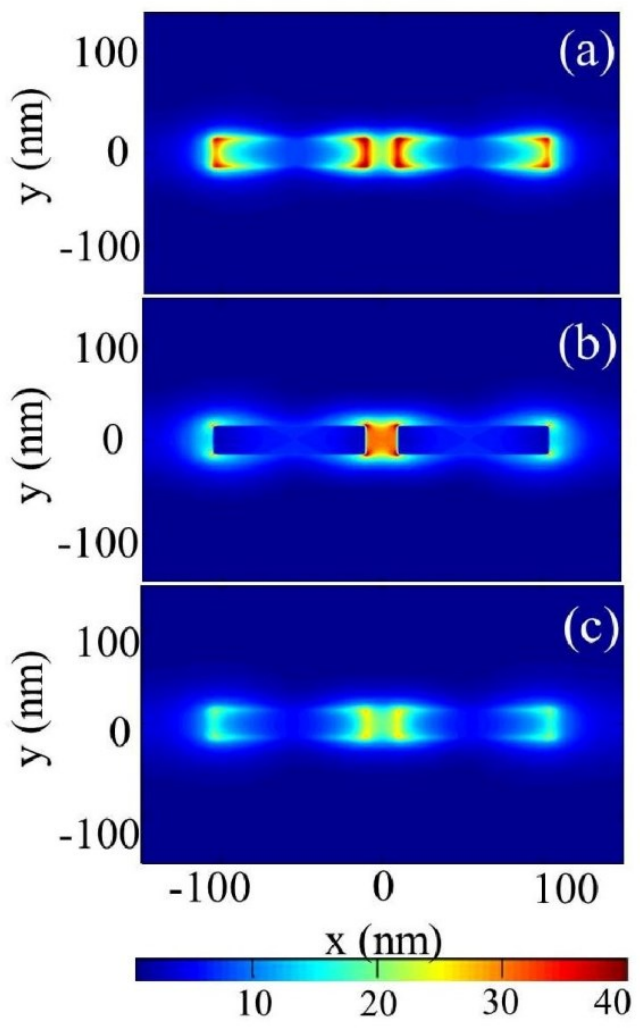

Fig. 2. Distribution of $|\mathbf{E}|=\sqrt{\left|\mathbf{E}_{\mathbf{X}}\right|^{2}+\left|\mathbf{E}_{\mathbf{y}}\right|^{2}+\left|\mathbf{E}_{\mathbf{z}}\right|^{2}}$ on $x-y$ cross-sectional planes for an Au dipole of dimensions $w=30 \mathrm{~nm}, t=30 \mathrm{~nm}, l=210 \mathrm{~nm}, g=20 \mathrm{~nm}$ and $p=q=300$ $\mathrm{nm}$ (a) $3 \mathrm{~nm}$ above the $\mathrm{Au} / \mathrm{Si}$ interface, (b) $15 \mathrm{~nm}$ above the $\mathrm{Au} / \mathrm{Si}$ interface, and (c) 3 $\mathrm{nm}$ above the Au surface in $\mathrm{H}_{2} \mathrm{O}$. Computations performed at $\lambda_{0}=1353 \mathrm{~nm}$ (resonance wavelength).

The transmittance $T$ is calculated as a function of frequency $(f)$ or wavelength via:

$$
T(f)=\frac{\int_{S} \operatorname{Re}\left(\mathbf{P}_{\mathbf{m}}\right) \cdot \mathbf{d} \mathbf{s}}{\int_{S} \operatorname{Re}\left(\mathbf{P}_{\mathbf{s}}\right) \cdot \mathbf{d} \mathbf{s}}
$$

where $\mathbf{P}_{\mathbf{m}}$ and $\mathbf{P}_{\mathbf{s}}$ are Poynting vectors at the location of the monitor where $T$ is calculated and at the location of the source, respectively. $S$ is the surface of the reference plane where the transmittance is calculated [28]. Eq. (1) can also be used to compute the reflectance $R$ of the system by replacing $\mathrm{S}$ with the appropriate reference plane.

Figure 3 plots the calculated transmittance $(T)$, reflectance $(R)$, absorptance $(A)$, and the electric field enhancement $E_{e n}$ of the array over wavelength, where $A$ is given by:

$$
A=1-T-R
$$

and the electric field enhancement $E_{\text {en }}$ is calculated at the center of the gap, $15 \mathrm{~nm}$ above the $\mathrm{Si} / \mathrm{H}_{2} \mathrm{O}$ interface, relative to the electric field at the same location in the absence of the antenna. 
From Fig.3 it is noted that $E_{\text {en }}$ peaks near resonance to a value of about 25. (Throughout this paper the resonant wavelength of the antennas $\left(\lambda_{0 r}\right)$ refers to the free-space wavelength at which the absorptance curve reaches its maximum value.) The misalignment of extrema in the $T, R$, and $A$ responses is due to absorption in Au. The absorptance of the system is due almost entirely to absorption in $\mathrm{Au}$ (losses are negligible in $\mathrm{Si}$ and $\mathrm{H}_{2} \mathrm{O}$ by comparison) [4].

One can relate the optical performance and response of the dipoles to their geometry via the full parametric study reported in [4]. Decreasing the gap size changes the transmittance and reflectance as the proportion of Au covering the surface changes. Also, decreasing the gap size increases the capacitance causing a red-shift in the resonance of the system. Finally, decreasing the gap size increases the electric field therein, which as we show below, increases the bulk and surface sensitivities of the dipoles.

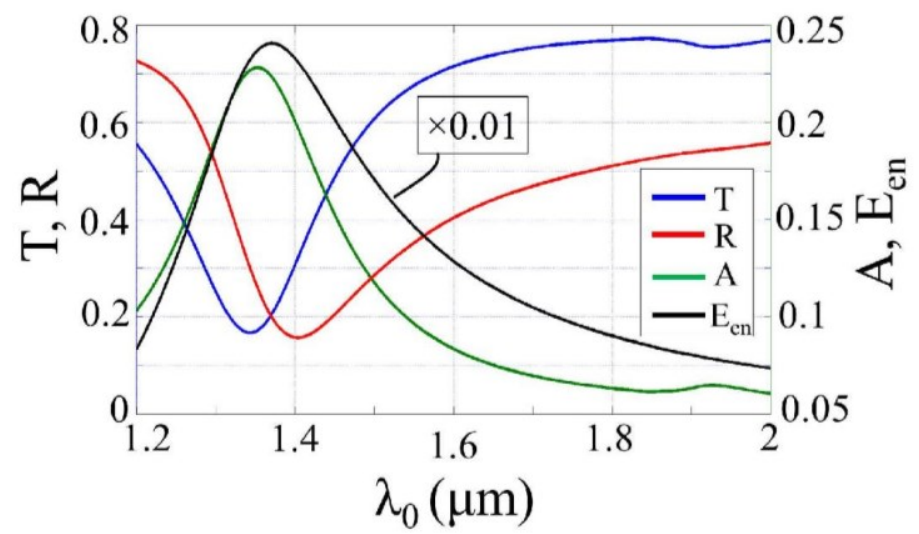

Fig. 3. Calculated transmittance $(T)$, reflectance $(R)$, absorptance $(A)$ and electric field enhancement $\left(E_{e n}\right)$ vs. free space wavelength $\left(\lambda_{0}\right)$.

\section{Quantum efficiency and responsivity}

The Au dipoles are assumed to form a Schottky contact to Si thereby creating an infrared photodetector operating on the basis of IPE (Fig. 1(a)). Hot (energetic) conduction carriers are created in the Au dipoles by absorption of SPPs resonating on the antennas. If the photon energy is greater than the Schottky barrier energy, then hot carriers may be emitted over the barrier and collected in the Si as photocurrent. The internal quantum efficiency $\eta_{i}^{t}$ is the number of hot carriers that contribute to the photocurrent per absorbed photon per second. We assumed $p-\mathrm{Si}$ because Schottky barriers are lower thereon than on $n$-Si, leading to increased quantum efficiency and detection at longer wavelengths. Also the effective Richardson coefficient for holes is lower than that for electrons which helps manage the dark current. We have used the thinfilm model described in [33] to estimate the internal quantum efficiency, taking the attenuation length of hot holes in Au as $55 \mathrm{~nm}$ [34] and the Schottky barrier height as $\phi_{B}=0.34 \mathrm{eV}$ for $\mathrm{Au}$ on $p$-Si [35]. Figure 4(a) shows $\eta_{i}^{t}$ computed over the wavelengths of interest.

Taking all $\mathrm{Au} / \mathrm{Si}$ contacts into consideration (a $25 \times 25$ network of dipoles and their related interconnects, and a circular contact pad of diameter $10 \mu \mathrm{m}$ ), a total contact area of $\sim 95.5 \times 10^{-12}$ $\mathrm{m}^{2}$ is obtained. Consequently, a dark current of $I_{d}=5.4 \mu \mathrm{A}$ is obtained [35] at $300 \mathrm{~K}$. The rectenna and interconnect area is $\sim 0.17 \times 10^{-12} \mathrm{~m}^{2}$ and the contact pad area is $95.45 \times 10^{-12}$ $\mathrm{m}^{2}$, so the pad area dominates; the dark current associated with the latter could be eliminated by inserting a dielectric between the pad and the Si surface. 
The responsivity $R_{e s p}$ is defined as the ratio of the photocurrent to the incident optical power and can be expressed in terms of $\eta_{i}^{t}$ as [36]:

$$
R_{e s p}=\kappa \frac{A \eta_{i}^{t} q}{h v}
$$

where $A$ is the absorptance, taken as that of the dipole array (Eq. (2)), and $\kappa$ is the fraction of the absorptance that contributes to the photocurrent [36] (taken as $\kappa=1$ herein). The minimum detectable power is given as the ratio of the dark current to the responsivity $S_{\min }=I_{d} / R_{e s p}$.
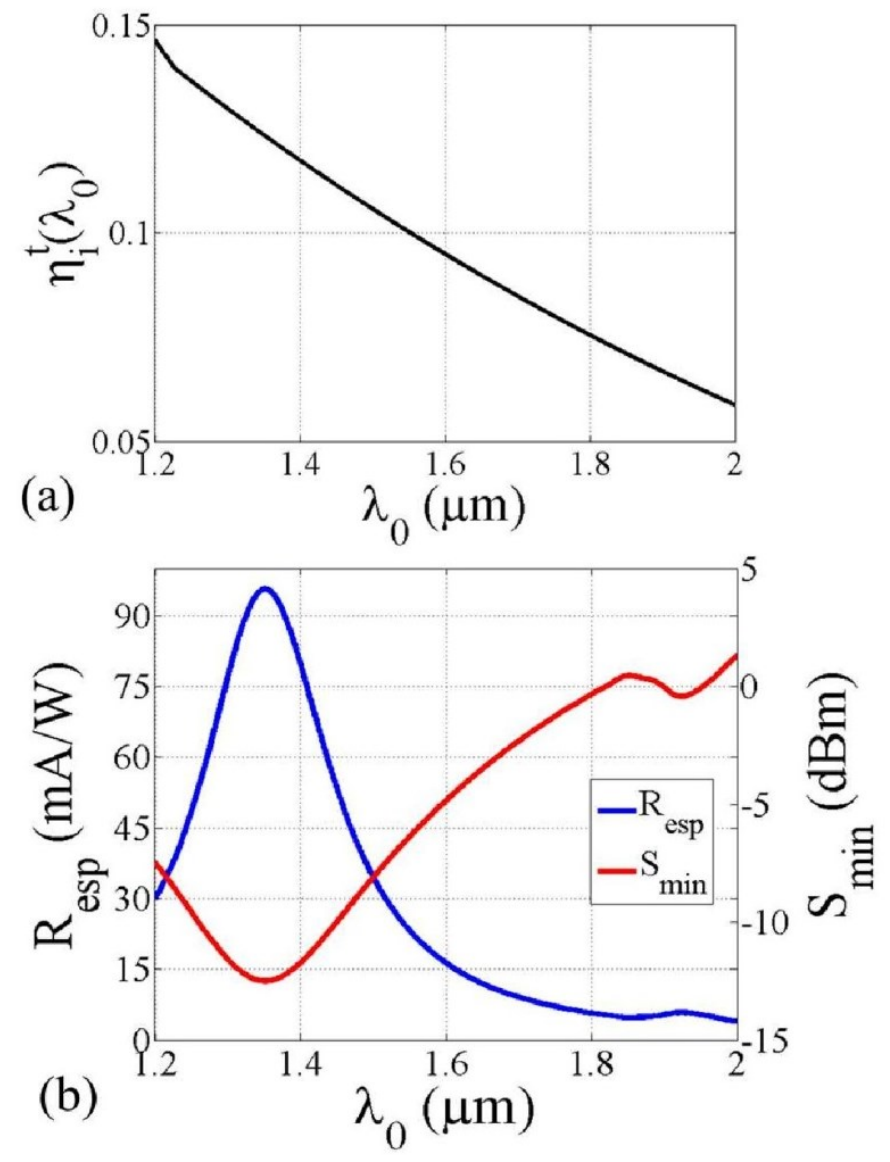

Fig. 4. (a) Internal quantum efficiency $\left(\eta_{i}^{t}\right)$ vs. $\lambda_{0}$. (b) Responsivity $\left(R_{\text {esp }}\right)$ and minimum detectable power $\left(S_{\min }\right)$ vs. $\lambda_{0}$.

The detection performance of the rectenna was assessed over a range of wavelengths and is plotted in Fig. 4(b). Compared to other SPP Schottky detectors, the responsivity and minimum detectable power of this detector are quite reasonable, even with a large contact pad [23,25]. This performance level is suitable for low-cost silicon based detection and for the intended biosensing, optical monitoring and interconnect applications. The wavelength response follows closely that of the absorptance of the dipole array (Fig. 4(b)), except that the former drops more rapidly at longer wavelengths due to the decrease in internal quantum efficiency $\left(\eta_{i}^{t}\right)$ as shown in Fig. 4(a). 


\section{Bulk sensitivity}

Monitoring changes in a surface plasmon resonance is a widely used interrogation approach for measuring changes in the refractive index of bulk solutions and changes in the thickness or composition of thin bio(chemical) adlayers on the biosensor surface.

For bulk sensing, the index of the cover material $n_{c}$ was changed from the nominal one $\left(\mathrm{H}_{2} \mathrm{O}\right)$ over a large range, and absorptance spectra were computed using the FDTD method and the same mesh size as in the first section of this paper $\left(0.5 \times 0.5 \times 0.5 \mathrm{~nm}^{3}\right)$. Figure $5(a)$ shows $A$ for all test cases considered. Increasing $n_{c}$ decreases $A$ and increases the electric field enhancement (not shown). The bulk sensitivity $\partial \lambda_{0 r} / \partial n_{c}$ of the rectenna and the peak responsivity $\left(R_{e s p, r}\right)$ are plotted in Fig. 5(b). The bulk sensitivity was computed by interpolating the spectral shifts using a cubic spline, then computing the derivative of the spline.
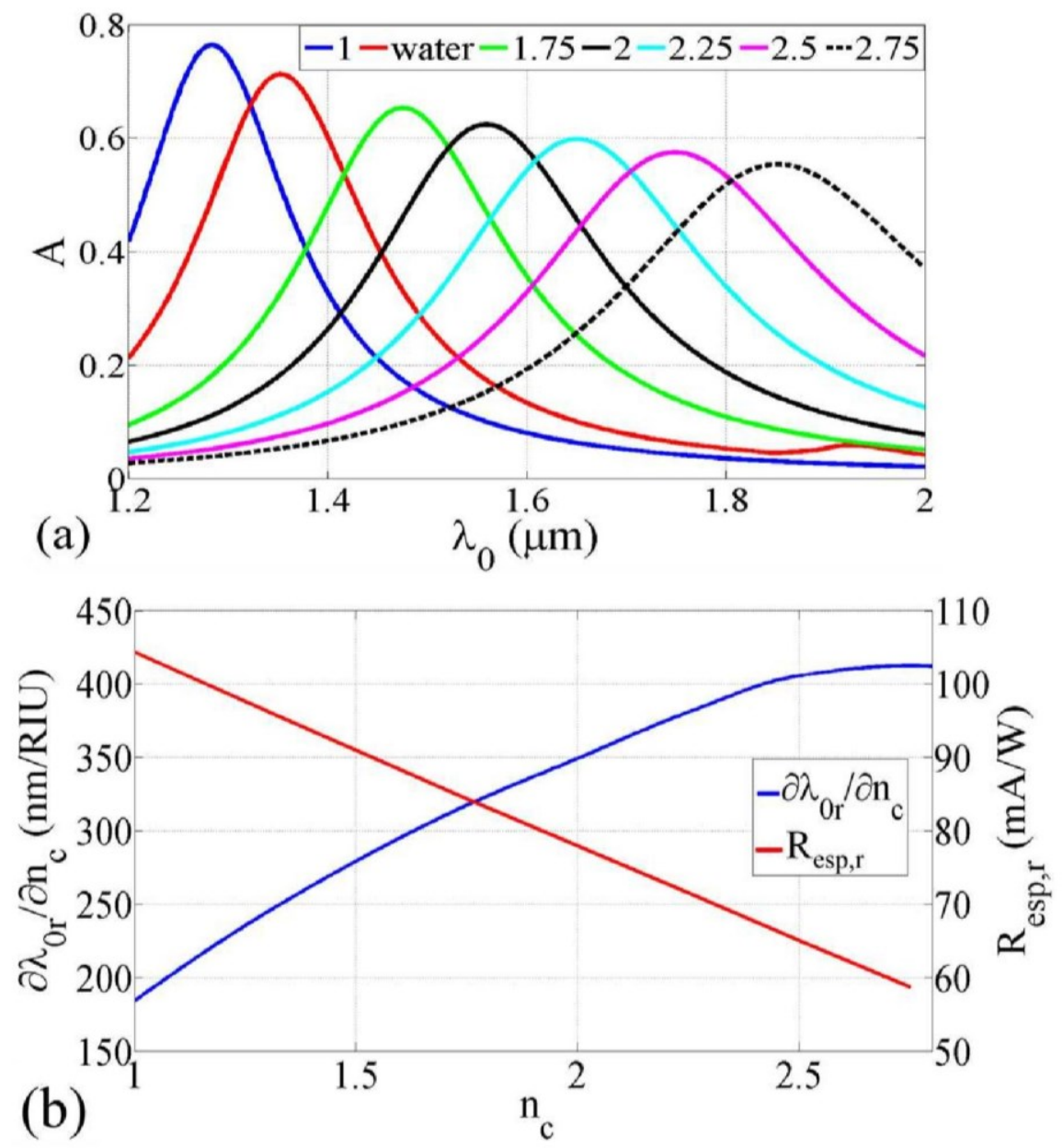

Fig. 5. (a) Absorptance $(A)$ vs. $\lambda_{0}$ for several cover refractive indices $n_{c}$ ranging from 1 to 2.75$)$. (b) Bulk sensitivity ( $\partial \lambda_{0 r} / \partial n_{c}-$ blue) and peak responsivity $\left(R_{e s p, r}-\right.$ red) of the rectenna as a function of $n_{c}$.

The rectenna has a bulk sensitivity comparable to what is reported in $[6,7,13]$. Recent analytical studies show that the bulk sensitivity increases with the surface plasmon resonance wavelength and the upper limit of this sensitivity is totally independent of nanostructure shape [37]; the

\#181367 - \$15.00 USD Received 10 Dec 2012; revised 17 Jan 2013; accepted 18 Jan 2013; published 12 Feb 2013 (C) 2013 OSA 
results of Fig. 5 are consistent with this trend. The peak responsivity drops linearly with $n_{c}$ following the peak absorptance, with the largest peak responsivity occurring for $n_{c}=1$. The drop in responsivity would translate to a commensurate drop in the monitored photocurrent as the bulk index changes. An incident optical power of $1 \mathrm{~mW}$ would yield a change in photocurrent of $\sim 40 \mu \mathrm{A}$ over the full index range, which is easily measureable.

\subsection{Waveguide modal analysis}

Modal analysis was carried out on the waveguide used as the arms of the dipoles using a mode solver based on the finite-difference method [26] in order to identify the mode propagating thereon. The wavelength for the modal analysis was set to $\lambda_{0}=1353 \mathrm{~nm}$, which is the resonant wavelength of the rectenna covered by $\mathrm{H}_{2} \mathrm{O}$. Figures 6(a) and 6(b) show the real part of the main transverse electric field $\left(E_{z}\right)$ of the mode, plotted over the $y-z$ cross-section of one of the dipole arms for the extreme cases of bulk index used in the sensitivity study, i.e., $n_{c}=1$ and $n_{c}=2.75$. The distribution of $E_{z}$ identifies the mode propagating along the nanowires as the $s a_{b}^{0}$ mode $[9,32]$, which is excited on the dipoles given the polarization, orientation and uniformity of the source field ( $x$-polarised plane wave). The field distribution is clearly perturbed by changes in the cover index (Fig. 6).

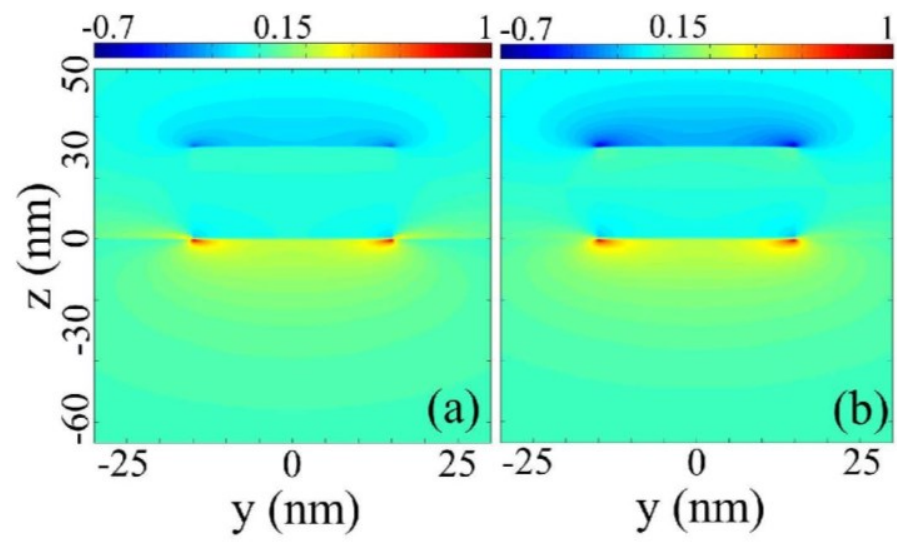

Fig. 6. Real part of $E_{z}$ of the $s a_{b}^{0}$ mode plotted over the cross-section of a nanowire waveguide $\left(\lambda_{0}=1353 \mathrm{~nm}\right)$ computed using a mode solver. (a) $n_{c}=1$ (air) and (b) $n_{c}=2.75$.

We compute the effective refractive index $\left(n_{\text {eff }}\right)$ and the mode power attenuation $(\alpha)$ of the $s a_{b}^{0}$ mode as a function of $n_{c}$ and plot the results in Fig. 7. Increasing $n_{c}$ increases the effective refractive index which causes, in part, the red shift observed in the absorptance curves of Fig. 5(a) $\left(\lambda_{0 r} \propto n_{c}\right)$; the red shift is also due to changes in the gap capacitance $C_{g}$ as $n_{c}$ changes. The mode power attenuation is also observed to increase with increasing $n_{c}$ explaining in part the broadening of the responses of Fig. 5(a) as $n_{c}$ increases.

\subsection{Analytical expression for the bulk sensitivity of dipoles}

An equivalent circuit was proposed in [4] to relate the resonant wavelength of dipole antennas to the effective index $\left(n_{\text {eff }}\right)$ of the $s a_{b}^{0}$ mode propagating along the nanowires forming the dipole, and to the characteristics of the dipole gap. We briefly summarize this circuit as it will be required in what follows. The circuit consists of two open-circuited transmission lines, one for each arm of the dipole, connected by a capacitor modeling the effects of the gap. The resonant 


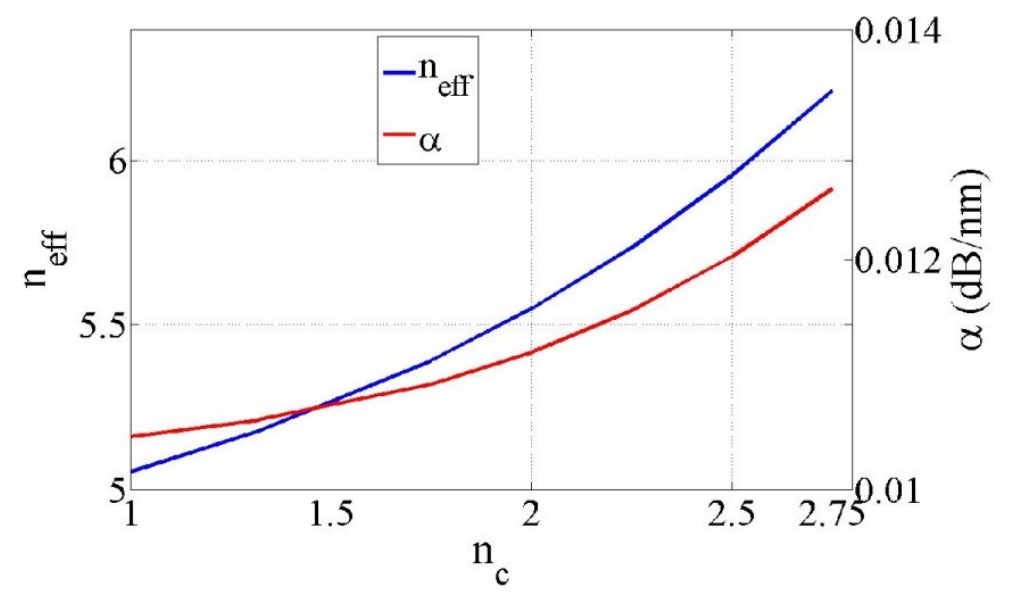

Fig. 7. Effective refractive index ( $n_{\text {eff }}$ blue) and mode power attenuation ( $\alpha$-red) of the $s a_{b}^{0}$ mode resonating along the dipoles as a function of $n_{c}$.

frequencies of dipole antennas $\omega_{0 r}$ satisfy the following transcendental equation derived for the circuit:

$$
\tan \left(n_{e f f} \omega_{0 r} \sqrt{\varepsilon_{0} \mu_{0}}\left(d+\delta_{m}\right)\right)=-2 \omega_{0 r} C_{g} Z_{0}
$$

where

$$
\omega_{0 r}=\frac{2 \pi c_{0}}{\lambda_{0 r}}
$$

In Eq. (4), $d+\delta_{m}$ is the effective length of each transmission line section, where $d=$ $(l-g) / 2$. The parameter $\delta_{m}$ is the distance from the end of each arm where the main field component decays to $1 / e$; in our dipole design $\delta_{m} \approx 21 \mathrm{~nm}$, taken as the decay of $E_{z}$ (the main transverse electric field component of the $s a_{b}^{0}$ mode - Fig. 6). Neglecting fringing fields, the capacitance of the gap $C_{g}$ is:

$$
C_{g}=\varepsilon_{c} \frac{A_{d}}{g}
$$

where $\varepsilon_{c}=\varepsilon_{0} \varepsilon_{r, c}$ and $\varepsilon_{r, c}$ are the absolute and relative permittivities of the material filling the gap at the wavelength of modal analysis, which is taken as a wavelength near the expected resonant wavelength of the dipole antenna. $A_{d}=w t$ is the cross-sectional area of the dipole arms. In Eq. (4), $Z_{0}$ is the characteristic impedance of the transmission lines calculated as:

$$
Z_{0}=\frac{\iint_{\infty} f(y, z) \operatorname{Re}\left[Z_{\omega}(y, z)\right] d S}{\iint_{\infty} f(y, z) d S}
$$

where $Z_{\omega}$ is the wave impedance of the $s a_{b}^{0}$ mode:

$$
Z_{\omega}=\frac{\hat{\mathbf{k}} \cdot\left(\mathbf{E} \times \mathbf{H}^{*}\right)}{(\hat{\mathbf{k}} \times \mathbf{H}) \cdot\left(\hat{\mathbf{k}} \times \mathbf{H}^{*}\right)}
$$

$\hat{\mathbf{k}}=\hat{\mathbf{x}}$ is the direction of modal propagation which is along the dipole axis, and $\mathbf{E}$ and $\mathbf{H}$ are the mode fields. In Eq. (7), $f(y, z)$ is a weighting function defined from the $s a_{b}^{0}$ mode fields as: 


$$
f(x, y)=\sqrt{\left|E_{y}(y, z)\right|^{2}+\left|E_{z}(y, z)\right|^{2}}
$$

Figure 8 plots the gap capacitance as a function of the bulk index. The red-shift in the absorptance curves are caused in part by the increase in $C_{g}$ which is noted to increase from 0.4 to $3 \mathrm{aF}$. Figure 8 also plots $Z_{0}$ as a function of $n_{c}$, showing a non-negligible variation (compared to the surface sensing case as discussed below).

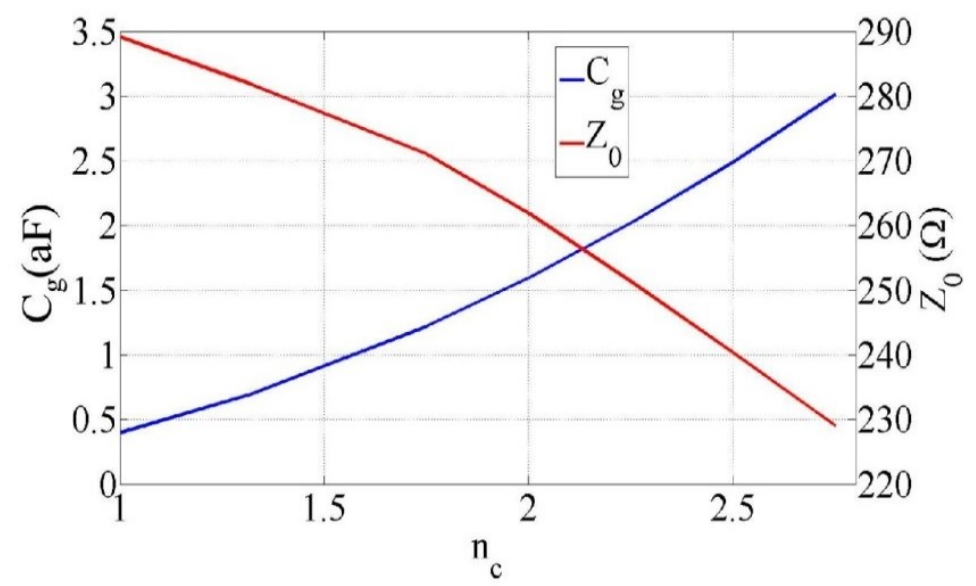

Fig. 8. Gap capacitance $C_{g}$ (blue) and characteristic impedance $Z_{0}$ of the $s a_{b}^{0}$ mode (red) as a function of $n_{c}$.

We use now the equivalent circuit model to gain insight on the bulk sensing performance of the dipoles. Taking the derivative of both sides of Eq. (4) with respect to nc yields:

$$
\begin{array}{r}
{\left[\left(d+\delta_{m}\right) \sqrt{\varepsilon_{0} \mu_{0}} \omega_{0 r} \frac{\partial n_{e f f}}{\partial n_{c}}+\left(d+\delta_{m}\right) \sqrt{\varepsilon_{0} \mu_{0}} n_{e f f} \frac{\partial \omega_{0 r}}{\partial n_{c}}\right] \times\left[1+\tan ^{2}\left(n_{e f f} \omega_{0 r} \sqrt{\varepsilon_{0} \mu_{0}}\left(d+\delta_{m}\right)\right]\right.} \\
=-2 \omega_{0 r} C_{g} \frac{\partial Z_{0}}{\partial n_{c}}-2 \omega_{0 r} Z_{0} \frac{\partial C_{g}}{\partial n_{c}}-2 C_{g} Z_{0} \frac{\partial \omega_{0 r}}{\partial n_{c}}
\end{array}
$$

where we note that $n_{\text {eff }}=n_{e f f}\left(n_{c}\right), \omega_{0 r}=\omega_{0 r}\left(n_{c}\right), \delta_{m}=\delta_{m}\left(n_{c}\right), C_{g}=C_{g}\left(n_{c}\right)$ and $Z_{0}=Z_{0}\left(n_{c}\right)$. In writing Eq. (10) we have supposed that $\partial \delta_{m} / \partial n_{c} \approx 0$, which is justified based on verifications carried out at our extreme values for $n_{c}$. After some manipulations, the following equation for the bulk sensitivity is obtained from the above:

$$
\zeta_{\omega} \frac{\partial \omega_{0 r}}{\partial n_{c}}=\zeta_{n} \frac{\partial n_{e f f}}{\partial n_{c}}+\zeta_{C} \frac{\partial C_{g}}{\partial n_{c}}+\zeta_{Z} \frac{\partial Z_{0}}{\partial n_{c}}
$$

where

$$
\begin{gathered}
\zeta_{\omega}=\left(\frac{d+\delta_{m}}{c_{0}}\right) n_{e f f}\left(1+4 \omega_{0 r}^{2} C_{g}^{2} Z_{0}^{2}\right)+2 C_{g} Z_{0} \\
\zeta_{n}=\left(\frac{d+\delta_{m}}{c_{0}}\right) n_{e f f}\left(1+4 \omega_{0 r}^{2} C_{g}^{2} Z_{0}^{2}\right) \\
\zeta_{C}=-2 \omega_{0 r} Z_{0}
\end{gathered}
$$




$$
\zeta_{\mathrm{Z}}=-2 \omega_{0 r} C_{g}
$$

and $c_{0}$ is the speed of light in vacuum. The term $\partial C_{g} / \partial n_{c}$ in Eq. (11) can be expressed in terms of dipole dimensions and gap material properties. Ignoring the imaginary part of the bulk material permittivity $\left(\varepsilon_{r, c} \approx n_{c}^{2}\right)$ this term can be simplified to:

$$
\frac{\partial C_{g}}{\partial n_{c}}=\left(\frac{w t}{g}\right) \frac{\partial \varepsilon_{c}}{\partial n_{c}}=2 \varepsilon_{0}\left(\frac{w t}{g}\right) n_{c}
$$

Substituting Eqs. (12) to (16) into Eq. (11), the expression for the bulk sensitivity becomes:

$$
\frac{\partial \omega_{0 r}}{\partial n_{c}}=-\zeta_{\omega}^{-1}\left[4\left(\frac{w t}{g}\right) \omega_{0 r} Z_{0}\right] n_{c}+\zeta_{\omega}^{-1}\left(\zeta_{n} \frac{\partial n_{e f f}}{\partial n_{c}}+\zeta_{Z} \frac{\partial Z_{0}}{\partial n_{c}}\right)
$$

The second term in Eq. (17) comes from mode properties and the first term is directly related to the gap capacitance. In Eq. (12), $2 C_{g} Z_{0} \ll\left(\left(d+\delta_{m}\right) / c_{0}\right) n_{e f f}\left(1+4 \omega_{0 r}^{2} C_{g}^{2} Z_{0}^{2}\right)$; neglecting this term and substituting in the expressions for $\zeta_{\omega}, \zeta_{n}$ and $\zeta_{Z}$ from Eqs. (12) to (15) into Eq. (17), leads to the simplified expression for the bulk sensitivity:

$$
\frac{\partial \omega_{0 r}}{\partial n_{c}} \approx-\left[\frac{2 w t c_{0} \omega_{0 r}}{g\left(d+\delta_{m}\right) n_{e f f}}\right] \frac{2 Z_{0} n_{c}+\frac{\partial Z_{0}}{\partial n_{c}} n_{c}^{2}}{1+\left(2 w t g^{-1} \omega_{0 r} Z_{0}\right)^{2} n_{c}^{2}}-\frac{\omega_{0 r}}{n_{e f f}} \frac{\partial n_{e f f}}{\partial n_{c}}
$$

The bulk sensitivity can be written in terms of the resonant wavelength using:

$$
\frac{\partial \lambda_{0 r}}{\partial n_{c}}=-\left(\frac{2 \pi c_{0}}{\omega_{0 r}^{2}}\right) \frac{\partial \omega_{0 r}}{\partial n_{c}}
$$

which yields

$$
\frac{\partial \lambda_{0 r}}{\partial n_{c}} \approx\left[\frac{2 w t c_{0} \lambda_{0 r}}{g\left(d+\delta_{m}\right) n_{e f f}}\right] \frac{2 Z_{0} n_{c}+\frac{\partial Z_{0}}{\partial n_{c}} n_{c}^{2}}{1+\left(4 \pi w t g^{-1} c_{0} \lambda_{0 r} Z_{0}\right)^{2} n_{c}^{2}}+\frac{\lambda_{0 r}}{n_{e f f}} \frac{\partial n_{e f f}}{\partial n_{c}}
$$

Figure 9(a) compares the resonant wavelengths computed using the FDTD method (Fig. 5(a)) with those generated by the transmission line model (Eq. (4) using the corresponding modal parameters $\left(n_{e f f}, Z_{0}\right)$ computed at the resonant wavelength of each FDTD test case. From Fig. 9(a) one observes that for small bulk indices, and perhaps for very large ones, the two curves diverge slightly from each other. However over most of the index range, the transmission line model agrees very well with the FDTD results. Based on these curves we compute $\partial \lambda_{0 r} / \partial n_{c}$ by numerical differentiation of cubic spline interpolants as in Fig. 5(b) and plot as Fig. 9(b). The black curve plots the bulk sensitivity calculated analytically using Eq. (20). For all values of $n_{c}$ the analytical solution (Eq. (20)) agrees very well with the transmission line model (Eq. 4), and both agree very well with the FDTD results for large values of $n_{c}$. The agreement deteriorates at lower values of $n_{c}$ which probably comes from neglecting $\partial \delta_{m} / \partial n_{c}$ and the fringing fields in the model for the gap capacitance (Eq. (6)). Still the transmission line model (Eq. (4)) and the analytical solution (Eq. (20)) can be used over a broad range of bulk indices to estimate performance rather than the more time-consuming FDTD computations.

One interesting and important property extracted from Fig. 9(b) is that we can fit a linear model to the black curve as $\partial \lambda_{0 r} / \partial n_{c} \approx 81 n_{c}+199$ (nm/RIU). Strictly, the slope and intercept of this linear model are functions of $\lambda_{0 r}$ and the modal parameters $n_{\text {eff }}$ and $Z_{0}$ which change with $n_{c}$; however, the model fits well over the range of $n_{c}$ investigated here. Comparing Eq. (20) with a linear model reveals that the slope term $\left(81 \mathrm{~nm} / \mathrm{RIU}^{2}\right)$ comes from the gap capacitance and the intercept term $(199 \mathrm{~nm} / \mathrm{RIU})$ mostly from perturbation of the mode fields.

\#181367 - \$15.00 USD Received 10 Dec 2012; revised 17 Jan 2013; accepted 18 Jan 2013; published 12 Feb 2013 (C) 2013 OSA 25 February 2013 / Vol. 21, No. 4 / OPTICS EXPRESS 4340 

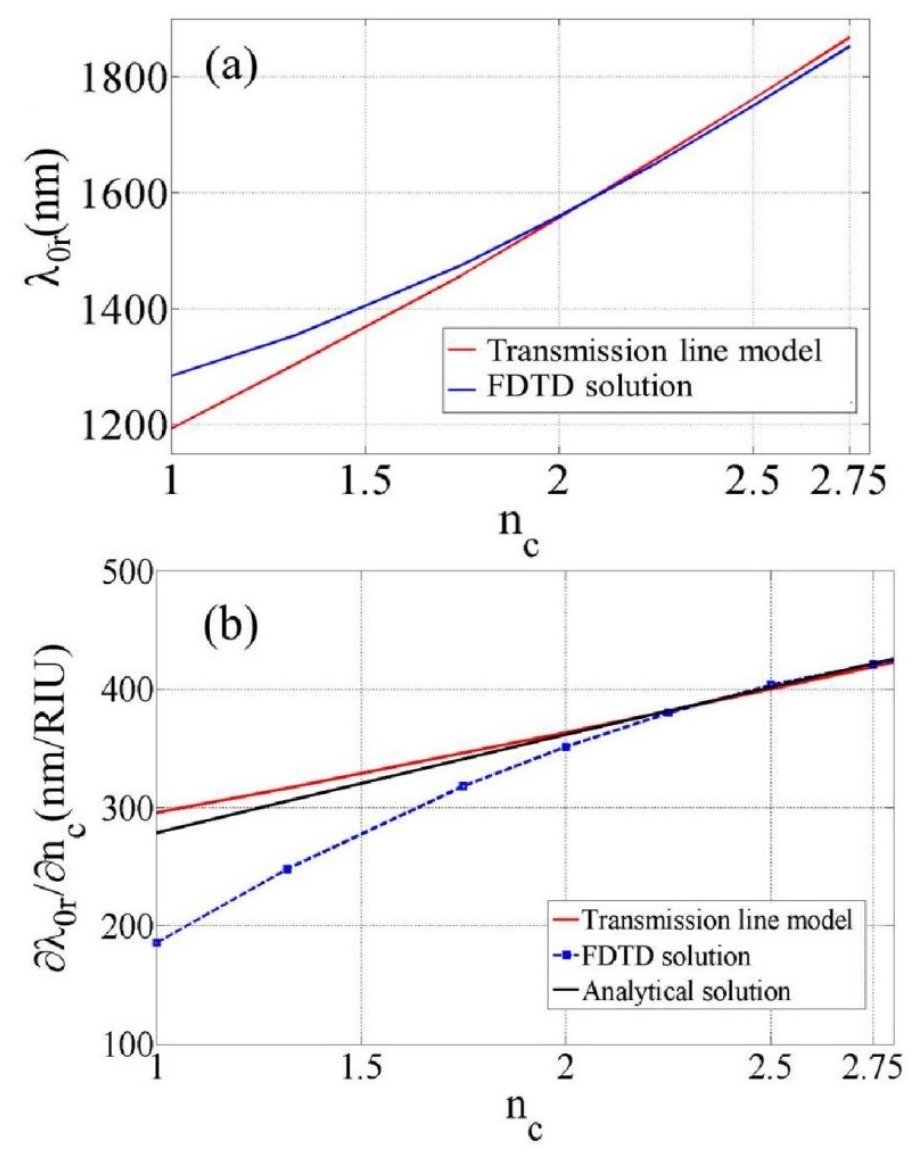

Fig. 9. (a) Resonant wavelengths computed using the transmission line model and the FDTD method as a function of bulk index $n_{c}$. (b) Bulk sensitivity computed using the FDTD method (dashed blue), the transmission line model (Eq. (4) - red), and the analytical solution (Eq. (20) - black).

\section{Surface sensitivity}

The surface sensitivity can be defined in a number of ways $[5,38,39]$. The definition adopted here is $\partial \lambda_{0 r} / \partial a$ where $a$ is the thickness of the adlayer of refractive index $n_{a}$ (Fig. 1(b)). The adlayer is modeled as uniform dielectric layer with its thickness and index $\left(a, n_{a}\right)$ being effective parameters [5]. The adlayer index is taken as $n_{a}=1.45$, which is representative of biochemical material [5], and it is assumed to grow on all $\mathrm{Au} / \mathrm{H}_{2} \mathrm{O}$ interfaces.

Previous studies $[4,40]$ indicate that the electric field enhancement is very sensitive to the gap size. Specifically, decreasing the gap increases the field enhancement in the gap, which in turn increases the overlap between the resonant mode and the adlayer, leading to increased sensitivity [41]. We therefore reduced the gap size from 20 to $10 \mathrm{~nm}$ and computed a series of absorptance curves as a increases from 0 to $5 \mathrm{~nm}$ using the FDTD method and a very small mesh size $\left(0.2 \times 0.2 \times 0.2 \mathrm{~nm}^{3}\right)$, as plotted in Fig. 10(a). For the case $a=5 \mathrm{~nm}$ the gap is fully loaded with adlayer and the shift in resonance relative to the no adlayer case $(a=0)$ is $30 \mathrm{~nm}$.

Fig. 10(b) gives $\partial \lambda_{0 r} / \partial a$ computed by interpolating the spectral shifts of Fig. 10(a) using a cubic spline, then computing the derivative of the spline. The largest surface sensitivity observed is $\partial \lambda_{0 r} / \partial a \sim 8 \mathrm{~nm} / \mathrm{nm}$, occurring in the early stages of adlayer growth, and then drop-

\#181367 - \$15.00 USD Received 10 Dec 2012; revised 17 Jan 2013; accepted 18 Jan 2013; published 12 Feb 2013 (C) 2013 OSA 
ping off significantly as the adlayer fills the gap region. The sensitivity is due mainly to the enhanced electric field in the gap and near the ends of each dipole where the enhanced fields overlap much more with the adlayer. Peak responsivity $\left(R_{e s p, r}\right)$ values are also plotted in Fig. 10(b); an incident optical power of $1 \mathrm{~mW}$ would yield a change in photocurrent of $\sim 1 \mu \mathrm{A}$ over the full adlayer range, which should be readily measureable.
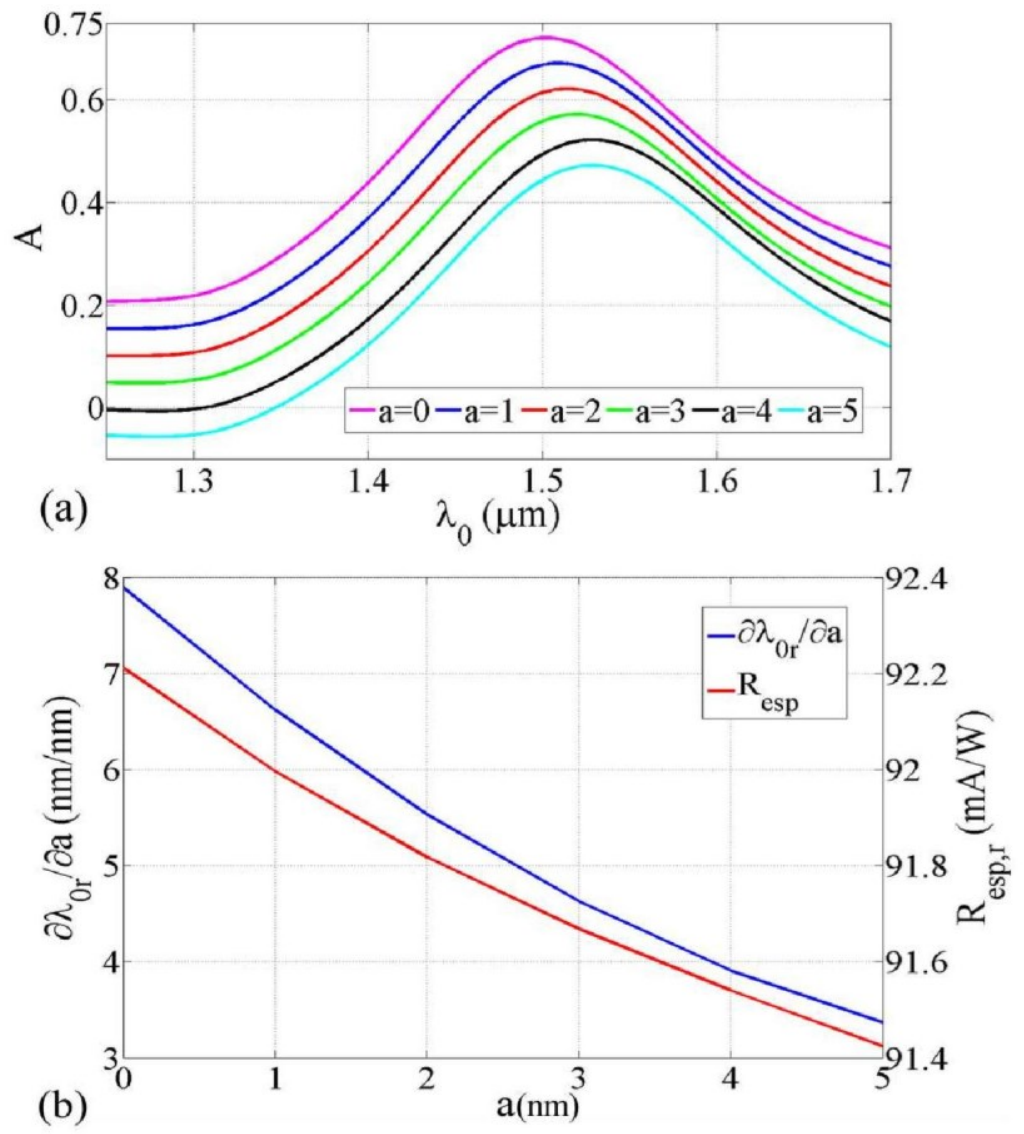

Fig. 10. (a) Absorptance $A$ vs. $\lambda_{0}$ for several adlayer thicknesses ( $a=0$ to $5 \mathrm{~nm}$ ); the curves are offset vertically by -0.05 for clarity. (b) Surface sensitivity $\left(\partial \lambda_{0 r} / \partial a\right.$ - blue) and peak responsivity ( $R_{\text {esp }, r}-$ red) as a function of $a$.

\subsection{Waveguide modal analysis}

Figure 11(a) and (b) show the real part of the main transverse electric field $\left(E_{z}\right)$ of the mode, plotted over the $y-z$ cross-section of one of the dipole arms for the extreme cases of surface adlayer thickness used in the sensitivity study, i.e., $a=0$ (no adlayer) and $a=5 \mathrm{~nm}$. The distribution of $E_{z}$ identifies the mode propagating along the nanowires as the $s a_{b}^{0}$ mode $[9,30]$, which is excited on the dipoles given the polarization, orientation and uniformity of the source field ( $x$-polarised plane wave). The field distribution is slightly perturbed by changes in the adlayer thickness (Fig. 11).

We have therefore computed the parameters of this mode $\left(n_{e f f}\right.$ and $\left.\alpha\right)$ as a function of a and we plot the results in Fig. 12. Increasing a decreases slightly $n_{\text {eff }}$ and $\alpha$ of the mode.

\#181367 - \$15.00 USD Received 10 Dec 2012; revised 17 Jan 2013; accepted 18 Jan 2013; published 12 Feb 2013 (C) 2013 OSA 


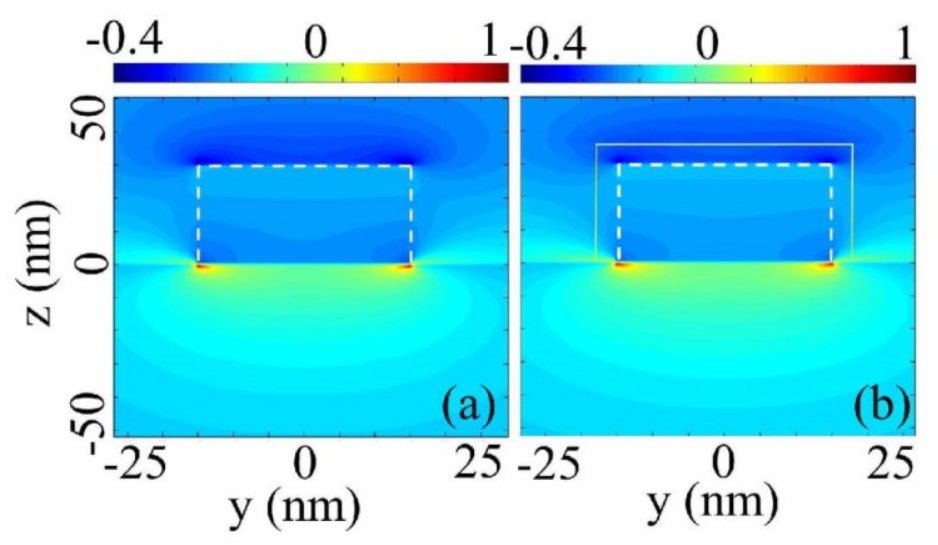

Fig. 11. Real part of $E_{z}$ of the $s a_{b}^{0}$ mode plotted over the cross-section of a nanowire waveguide $\left(\lambda_{0}=1353 \mathrm{~nm}\right)$ computed using a mode solver. (a) $a=0$ (no adlyaer) and (b) $a=5$ $\mathrm{nm}$.

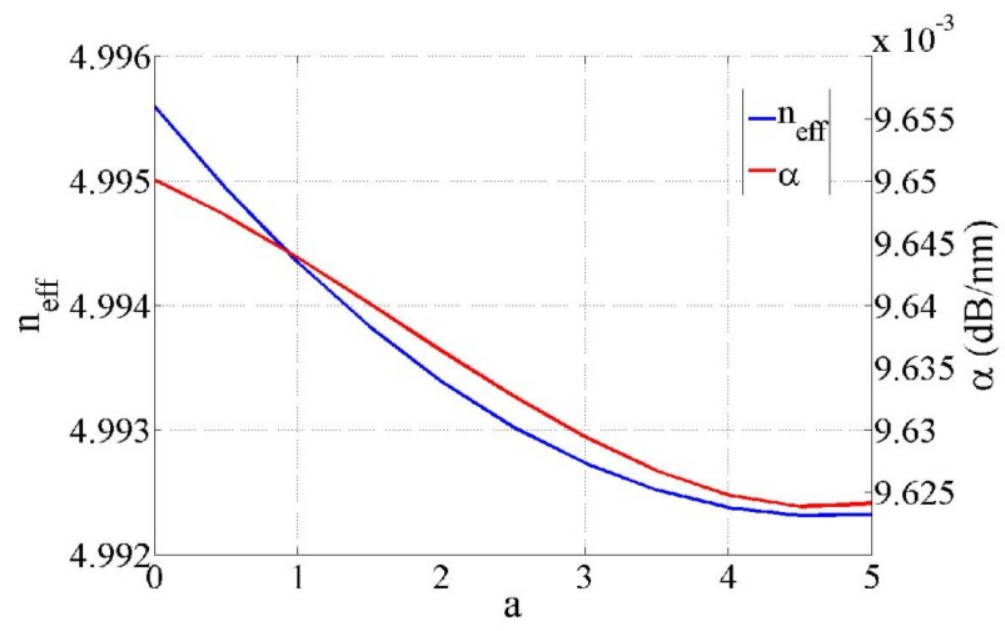

Fig. 12. Effective refractive index ( $n_{\text {eff }}$ blue) and mode power attenuation ( $\alpha$ - red) of the $s a_{b}^{0}$ mode resonating along the dipoles as a function of $a$.

\subsection{Analytical expression for the surface sensitivity of dipoles}

The red shift in the absorptance curves is due to changes in the parameters of the $s a_{b}^{0}$ mode resonating along the dipoles (Fig. 12) and to changes in the gap capacitance as the adlayer grows. The equivalent transmission line circuit described in the previous section can be used to take both effects into account using a new expression for $C_{g}$. Adding the adlayer produces three capacitors in series within the gap. Two capacitors $\left(C_{1}\right)$ are due to the adlayers growing on the ends of the right and left arms, and the third $\left(C_{2}\right)$ is formed by $\mathrm{H}_{2} \mathrm{O}$ filling the gap, as sketched in Fig. 13.

From circuit theory, the total gap capacitance is calculated as:

$$
C_{g}=\left(2 C_{1}^{-1}+C_{2}^{-1}\right)^{-1}
$$

where $C_{1}$ and $C_{2}$ are: 


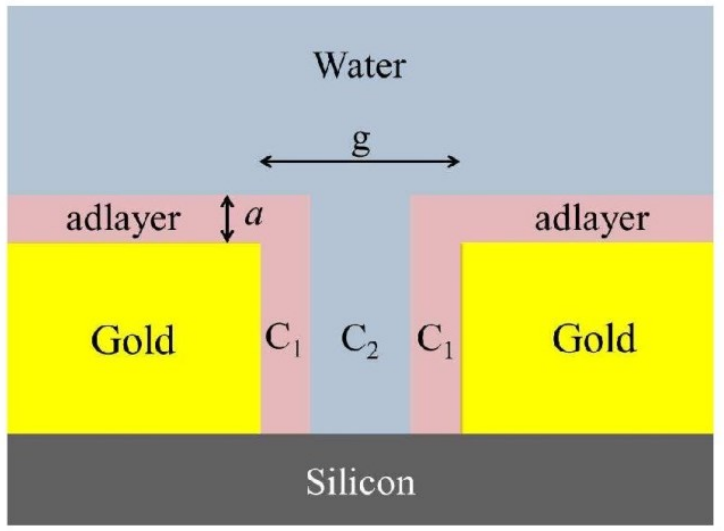

Fig. 13. Schematic of a dipole gap showing three plate capacitances in series.

$$
C_{1}=\varepsilon_{a}\left(\frac{w t}{a}\right)
$$

and

$$
C_{2}=\varepsilon_{c}\left(\frac{w t}{g-2 a}\right)
$$

In the above, $\varepsilon_{c}=\varepsilon_{0} \varepsilon_{r, c}$ and $\varepsilon_{a}=\varepsilon_{0} \varepsilon_{r, a}$ are the absolute permittivities of the bulk solution $\left(\mathrm{H}_{2} \mathrm{O}\right)$ and the adlayer, respectively. We ignore the imaginary parts of the permittivities and take $\varepsilon_{r, c} \approx n_{c}^{2} \approx 1.32^{2} \approx 1.74$ and $\varepsilon_{r, a} \approx n_{a}^{2} \approx 1.45^{2} \approx 2.11$. After some manipulations, we write $C_{g}$ as:

$$
C_{g}=\frac{\varepsilon_{a} \varepsilon_{c} w t}{2\left(\varepsilon_{a}-\varepsilon_{c}\right) a+\varepsilon_{a} g}
$$

Figure 14 plots $C_{g}$ (Eq. (24) and $Z_{0}$ of the $s a_{b}^{0}$ mode (Eqs. (7) to (9) as a function of the adlayer thickness $a$, showing that $C_{g}$ increases and $Z_{0}$ decreases slightly with $a$.

Taking the derivative of Eq. (4) with respect to $a$ yields:

$$
\zeta_{\omega} \frac{\partial \omega_{0 r}}{\partial a}=\zeta_{n} \frac{\partial n_{e f f}}{\partial a}+\zeta_{C} \frac{\partial C_{g}}{\partial a}+\zeta_{Z} \frac{\partial Z_{0}}{\partial a}
$$

where $\zeta_{\omega}, \zeta_{n}, \zeta_{C}$ and $\zeta_{Z}$ are defined in Eqs. (12) to (15). The term $\partial C_{g} / \partial a$ in Eq. (25) can be evaluated using Eq. (24) and expressed in terms of dipole dimensions and the properties of the materials filling the gap (emphi.e., the adlayer and $\mathrm{H}_{2} \mathrm{O}$ ) as:

$$
\frac{\partial C_{g}}{\partial a}=\frac{2 \varepsilon_{0} \varepsilon_{r, c} \varepsilon_{r, a}\left(\varepsilon_{r, a}-\varepsilon_{r, c}\right) w t}{\left[2\left(\varepsilon_{r, a}-\varepsilon_{r, c}\right) a+\varepsilon_{r, a} g\right]^{2}}
$$

The above reveals that $\partial C_{g} / \partial a$ is a saturating nonlinear function of the adlayer thickness $a$. Substituting Eq. (26) into Eq. (25), we obtain the following expression for the surface sensitivity as a function of $a$ :

$$
\frac{\partial \omega_{0 r}}{\partial a}=\frac{2 \varepsilon_{0} \varepsilon_{r, c} \varepsilon_{r, a}\left(\varepsilon_{r, a}-\varepsilon_{r, c}\right) w t}{\left[2\left(\varepsilon_{r, c}-\varepsilon_{r, a}\right) a+\varepsilon_{r, a} g\right]^{2}} \zeta_{\omega}^{-1} \zeta_{C} \omega_{0 r} Z_{0}+\zeta_{\omega}^{-1}\left(\zeta_{n} \frac{\partial n_{e f f}}{\partial a}+\zeta_{Z} \frac{\partial Z_{0}}{\partial a}\right)
$$




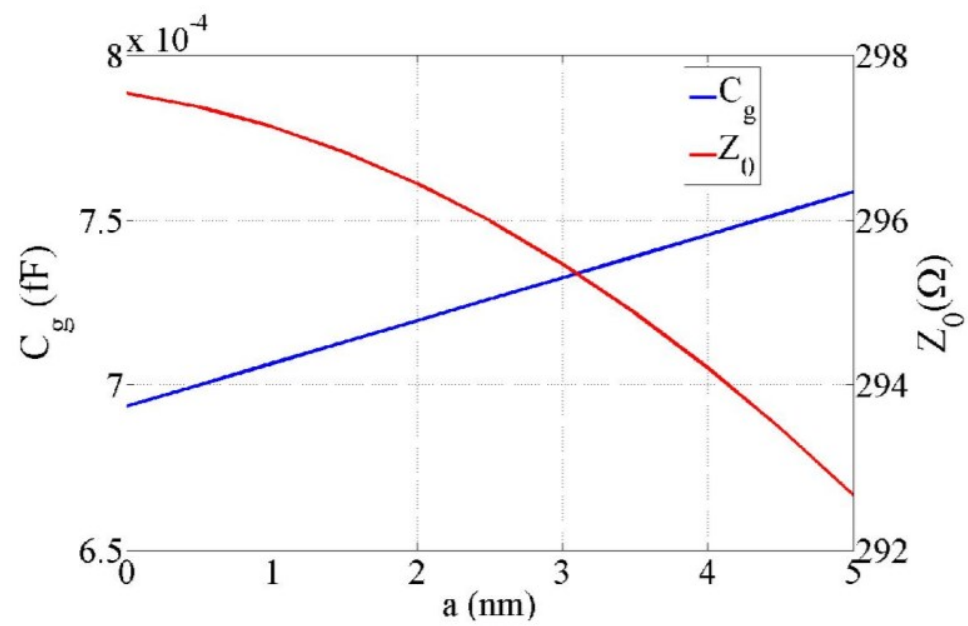

Fig. 14. Gap capacitance $C_{g}$ (blue) and characteristic impedance $Z_{0}$ of the $s a_{b}^{0}$ mode (red) as a function of $a$.

The surface sensitivity can be written in terms of the resonant wavelength using:

$$
\frac{\partial \lambda_{0 r}}{\partial a}=-\left(\frac{2 \pi c_{0}}{\omega_{0 r}^{2}}\right) \frac{\partial \omega_{0 r}}{\partial a}
$$

which yields:

$$
\frac{\partial \lambda_{0 r}}{\partial a}=\frac{4 \varepsilon_{0} \varepsilon_{r, c} \varepsilon_{r, a}\left(\varepsilon_{r, c}-\varepsilon_{r, a}\right) w t}{\left[2\left(\varepsilon_{r, c}-\varepsilon_{r, a}\right) a+\varepsilon_{r, a} g\right]^{2}} \zeta_{\omega}^{-1} \zeta_{C} \lambda_{0 r} Z_{0}-\zeta_{\omega}^{-1} \frac{\lambda_{0 r}^{2}}{2 \pi c_{0}}\left(\zeta_{n} \frac{\partial n_{e f f}}{\partial a}+\zeta_{Z} \frac{\partial Z_{0}}{\partial a}\right)
$$

From Fig. 12, it is clear that $\partial n_{e f f} / \partial a$ and $\partial Z_{0} / \partial a$ are approximately constant $(\sim-0.0014$ $\mathrm{nm}^{-1}$ and $1 \Omega / \mathrm{nm}$ ) over the range of a considered. Thus from Eq. (29) we find that $\partial \lambda_{0 r} / \partial a$ is also a saturating nonlinear function of a (which follows from Eq. (24)).

Figure 15(a) compares the resonant wavelengths obtained via the FDTD (Fig. 10(a)) and with those obtained by solving Eq. (4) using the corresponding modal parameters $\left(n_{e f f}, Z_{0}\right)$ computed at the resonant wavelength of every FDTD test case. Based on these results we compute $\partial \lambda_{0 r} / \partial a$ (by numerical differentiation of spline interpolants) and plot this as Fig. 15(b). The black curve plots the surface sensitivity calculated using the analytical solution, Eq. (29). For all adlayer thicknesses, the analytical solution (Eq. (29)) agrees very well with the transmission line model (Eq. (4)), and both are in reasonable agreement with the FDTD computations. The error probably comes from neglecting $\partial \delta_{m} / \partial a$ and the fringing fields in the model for the gap capacitance (Eq. (24)). Still the transmission line model (Eq. (4)) and the analytical solution (Eq. (29)) can be used over a broad range of adlayer thicknesses to estimate the surface sensitivity of dipoles rather than the more time-consuming FDTD method. The results from the transmission line model differ from the FDTD results by a maximum of $25 \%$.

\section{Comparison of dipole and monopole sensitivities}

Using the transmission line model we derive the bulk and surface sensitivities of monopole antennas and compare them to those of dipole antennas. Replacing all dipoles with monopoles of comparable length $(d=2 l$ and $g=0)$, we find the following equation for the resonant wavelengths of the equivalent transmission line circuit:

\#181367 - \$15.00 USD Received 10 Dec 2012; revised 17 Jan 2013; accepted 18 Jan 2013; published 12 Feb 2013

(C) 2013 OSA

25 February 2013 / Vol. 21, No. 4 / OPTICS EXPRESS 4345 

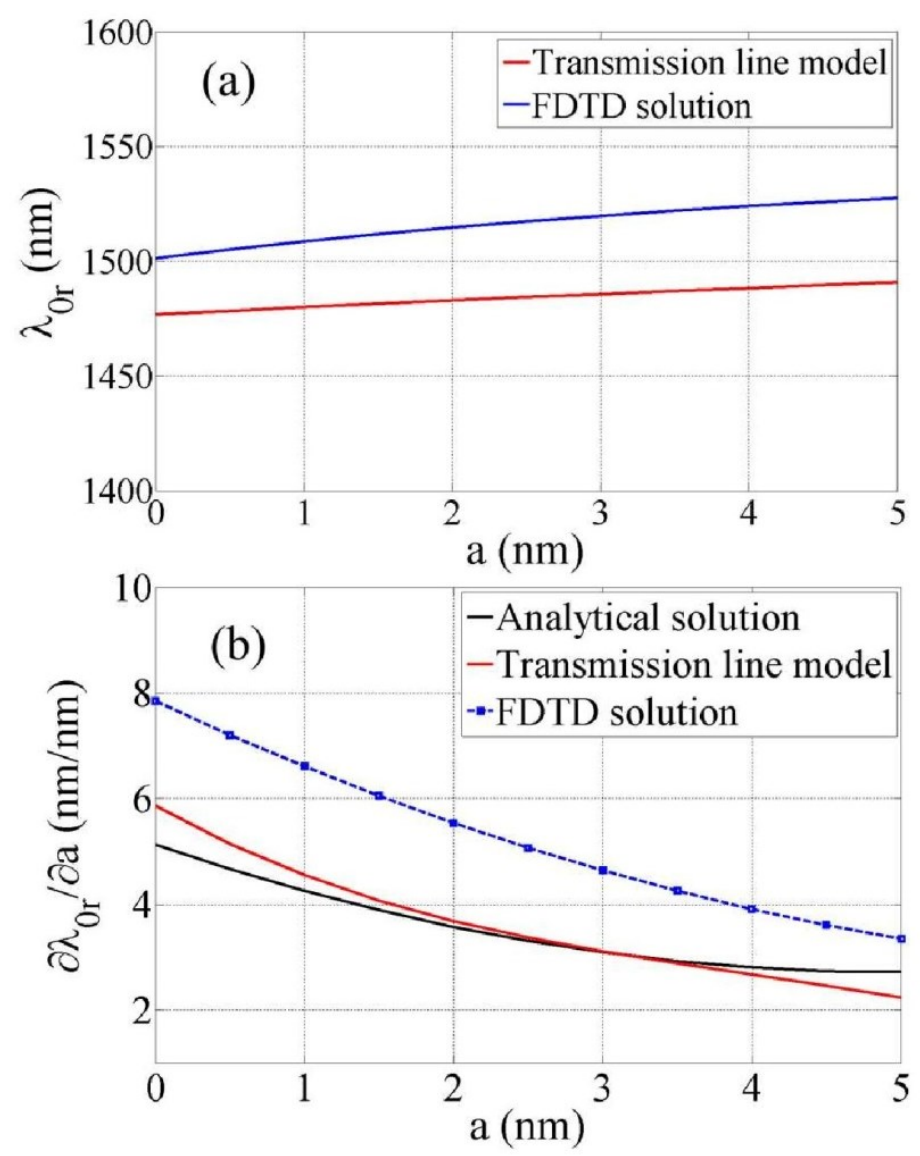

Fig. 15. (a) Resonant wavelengths computed using the transmission line model and the FDTD method as a function of adlayer thickness $a$. (b) Surface sensitivity computed using the FDTD method (dashed blue), the transmission line model (Eq.(4) - red), and the analytical solution (Eq.(29) - black).

$$
\cot \left(n_{\text {eff }} \omega_{0 r} \sqrt{\varepsilon_{0} \mu_{0}}\left(d+\delta_{m}\right)\right)=0
$$

where the symbols retain the same meaning as in Eq. (11). We note, of course, that there is no lumped capacitance in this model because there is no gap. By taking the derivative of the above with respect to $n_{c}$ and a we find the following expressions for the bulk and surface sensitivities:

$$
\begin{gathered}
\frac{\partial \lambda_{0 r}}{\partial n_{c}}=\frac{\lambda_{0 r}}{n_{e f f}} \frac{\partial n_{e f f}}{\partial n_{c}} \\
\frac{\partial \lambda_{0 r}}{\partial a}=\frac{\lambda_{0 r}}{n_{e f f}} \frac{\partial n_{e f f}}{\partial a}
\end{gathered}
$$

We note that a monopole corresponds to a dipole in the limit of $g \rightarrow 0$, which leads to an infinite gap capacitance, i.e. $C_{g} \rightarrow \infty$. In this limit, $\zeta_{\omega}, \zeta_{n}$ and $\zeta_{Z}$ become very large (Eqs. (13), (14) and (15)) and Eqs. (20) and (29) simplify to Eqs. (31) and (32).

Comparing Eqs. (31) and (32) with the corresponding ones for dipoles (Eqs. (20) and (29)), we observe that the sensitivities of monopole antennas are smaller than those of dipole antennas.

\#181367 - \$15.00 USD Received 10 Dec 2012; revised 17 Jan 2013; accepted 18 Jan 2013; published 12 Feb 2013

(C) 2013 OSA

25 February 2013 / Vol. 21, No. 4 / OPTICS EXPRESS 4346 
This is due to the absence of the gap in the former; thus, monopole resonances are altered only by changes in the properties of the $s a_{b}^{0}$ mode resonating on the antenna, whereas in dipoles, changes in the cap capacitance lead to a larger shift in resonance. Also, the electric field is strongly enhanced in the gap of dipoles compared to other locations on the antenna. The bulk sensitivity of dipoles is at least $1.65 \times$ greater than that of comparable monopoles. Similarly the surface sensitivity of dipoles is at least $1.5 \times$ greater than that of monopoles. In both cases, the importance of the gap is manifest.

\section{Conclusion}

Arrays of Au nano-dipole antennas forming a Schottky contact to Si were proposed and investigated theoretically for use as a wavelength-selective sub-bandgap rectenna and biosensor. Photodetection occurs via the internal photoelectric effect for photon energies above the Schottky barrier height but below the bandgap of Si. Excitation by a normally-incident plane wave polarized along the length of the dipoles was considered. Under this excitation, non-invasive electrical contacts to each dipole arm can be introduced, facilitating collection of the photocurrent. The plane-wave source excites a specific strongly-confined SPP mode ( $\left.s a_{b}^{0}[9]\right)$ propagating along the arms of the dipole antennas, forming a bonding resonance as the main resonance of operation of the antennas. Utilizing a Schottky detector structure simplifies interrogation in that the transmittance or reflectance need not be measured; rather only the photocurrent collected at the contacts needs to be monitored. Illuminating the antennas through the substrate leaves the top side of the device available for integrating microfluidics.

As a rectenna, high responsivities $(100 \mathrm{~mA} / \mathrm{W})$ and practical minimum detectable powers $(-12 \mathrm{dBm})$ are predicted at wavelengths near $1310 \mathrm{~nm}$. As a biosensor, the rectenna structure offers significant advantages as it simplifies the interrogation set-up: only the photocurrent generated by the device need be monitored, and the excitation optics (bottom) are physically separated from the micro-fluidics (top) by the device. Compelling sensitivities are predicted for the dipoles: $250 \mathrm{~nm} / \mathrm{RIU}$ in bulk sensitivity and $8 \mathrm{~nm} / \mathrm{nm}$ in surface sensitivity (biochemical adlayer in $\mathrm{H}_{2} \mathrm{O}$ ). In terms of changes in photocurrent, an incident optical power of $1 \mathrm{~mW}$ would yield a change of $\sim 1 \mu \mathrm{A}$ as an adlayer grows to $5 \mathrm{~nm}$ in thickness, which is readily measureable.

Using a transmission line model to represent the dipole antennas, we derive analytical expressions for the bulk and surface sensitivities of the structure and validate them via comparisons with numerical results. We proved using these expressions that dipole antennas provide much greater bulk and surface sensitivities than monopole antennas because of the existence of the gap in the former, which introduces a capacitance (and enhanced fields) that strongly affects the resonance.

\#181367 - \$15.00 USD Received 10 Dec 2012; revised 17 Jan 2013; accepted 18 Jan 2013; published 12 Feb 2013

(C) 2013 OSA

25 February 2013 / Vol. 21, No. 4 / OPTICS EXPRESS 4347 


\section{Chapter: Optimization of Plasmonic Nanoantennas for Sensing Applications: Consideration of Materials and Wavelengths}

The following article entitled "Optimization of Plasmonic Nanodipole Antenna Arrays for Sensing Application, was published in IEEE Journal of Selected Topics in Quantum Electronics, Volume 20, Issue 3 on May 2014 and is presented in full. The article represents work performed solely by the primary author under the supervision and guidance of the co-authors.

In this manuscript, authors present the optimization process for a plasmonic nanodipole antenna array designed and discussed in chapter 4 . The nanoantenna supports short-range surface plasmon polaritons (SPPs), in order to improve its sensing properties such as bulk sensitivity. Authors supposed that the array is integrated on a commercially used substrate (either silicon or glass) and covered by water which selected in anticipation of an eventual biosensing application.

Using modal analysis, a full parametrical study has been carried out on the dimensions of nanodipoles in three relevant wavelengths, $850 \mathrm{~nm}, 1310 \mathrm{~nm}$ and $1550 \mathrm{~nm}$. The optimized values were found to get more bulk sensitivity; results show a good performance over a wide range for nanodipole dimensions which help in the fabrication of the sensor. They show that the sensor with glass substrate gives more bulk sensitivity than the silicon type. Maximum bulk sensitivity is predicted at wavelength of $1550 \mathrm{~nm}$ as of $1000 \mathrm{~nm} / \mathrm{RIU}$ for the glass substrate type and 500 $\mathrm{nm} / \mathrm{RIU}$ for the silicon substrate type. Finally, some of the results are validated using full 3D FDTD solutions. 


\title{
Optimization of Plasmonic Nanodipole Antenna Arrays for Sensing Applications
}

\author{
Mohammad Alavirad, Langis Roy, Associate Member, IEEE, and Pierre Berini, Fellow, IEEE
}

\begin{abstract}
Nanoantennas are key optical components for several applications including biosensing. This paper presents the optimization of plasmonic nanodipole antenna arrays, operating with short-range surface plasmon polaritons, to maximize bulk sensitivity. The array is integrated on a substrate (silicon or glass) and covered by water. Using modal analysis, a full study was carried out on the dimensions of the nanodipoles at three optical wavelengths of interest, 850,1310 , and $1550 \mathrm{~nm}$, and some of the results were validated using full 3D FDTD modeling. We show that nanodipoles on a glass substrate produce a greater bulk sensitivity than on a silicon substrate. The largest bulk sensitivities are produced at the longest wavelength $(1550 \mathrm{~nm})$ as $1000 \mathrm{~nm} / \mathrm{RIU}$ on glass and $500 \mathrm{~nm} / \mathrm{RIU}$ on silicon. Good performance over a wide range of nanodipole dimensions was observed, making the arrays tolerant to imperfections.
\end{abstract}

Index Terms-Bionanotechnology, biosensing, antenna array, nanodipole, photonics, plasmonics, surface plasmon polariton.

\section{INTRODUCTION}

$\mathbf{S}$ URFACE plasmon polariton (SPP) sensors are widely used to detect molecular interactions. They are capable of monitoring the refractive index at the metal surfaces [1]. A new configuration for SPP sensors, based on metal nanoantennas [2]-[4], provides high bulk and surface sensitivities, making them useful for simple and fast label-free biodetection [5]. Localized surface plasmons (LSPs) on nanoparticles are contextually similar; their sensitivities to the local environment have been exploited in bio(chemical) sensors [6] and the effects of nanoparticle size, shape, and composition have been studied [7], [8]. Two interrogation approaches widely used for conventional SPP sensing are angular and spectral interrogation [1]. The first one involves measuring the change in resonance angle due to refractive index changes using a fixed wavelength source, while the latter uses a light source at a fixed angle and the index changes are monitored by the changes in the resonance wavelength [9]. These

Manuscript received August 6, 2013; revised October 10, 2013; accepted October 28, 2013.

M. Alavirad is with the Department of Electronics, Carleton University, Ottawa, ON K1S 5B6, Canada, and also with the School of Electrical Engineering and Computer Science, University of Ottawa, Ottawa, ON K1N 6N5, Canada (e-mail: alavirad@doe.carleton.ca).

L. Roy is with the Department of Electronics, Carleton University, Ottawa, ON K1S 5B6, Canada (e-mail: 1roy@ doe.carleton.ca).

P. Berini is with the School of Electrical Engineering and Computer Science, and also with the Department of Physics, University of Ottawa, Ottawa, ON K1N 5N4, Canada (e-mail: berini@eecs.uottawa.ca).

Color versions of one or more of the figures in this paper are available online at http://ieeexplore.iee.org.

Digital Object Identifier 10.1109/JSTQE.2013.2289963 interrogation approaches can also be used with nanoantenna arrays.

Many works have investigated the dependency of nanostructure sensitivity on geometry. Kavasnicka and Homola explored the bulk sensitivity of LSPs on gold nanoparticles using a dielectric overlayer, and found that it decreases with increasing particle size for particle diameters up to about $80 \mathrm{~nm}$ and then increases with increasing particle size [10]. The maximum bulk sensitivity found was $185 \mathrm{~nm} / \mathrm{RIU}$ for an overlayer thickness of $20 \mathrm{~nm}$ and a particle diameter of $160 \mathrm{~nm}$. Chen et al. measured the response of the surface plasmon peak of a set of gold nanoparticles of different shapes and found that the bulk sensitivities generally increase as Au nanoparticles become elongated and their apex become sharper [11]. They obtained bulk sensitivities [nm/RIU] of 44 for nanospheres, 83 for nanucubes, 150 to 540 for nanobipyramids of different aspect ratios, 195 to 288 for nanorods of different aspect ratios and up to 703 for nanobranches. In [12], the sensitivity of Ag strip pair arrays is discussed, where it is shown that the bulk sensitivity decreases from $700 \mathrm{~nm} / \mathrm{RIU}$ to $530 \mathrm{~nm} / \mathrm{RIU}$ as the slit width increases. In [13], Tsai et al. described a sensor consisting of square-lattice slab-like gold nanorings, and investigated the sensitivity of its bonding mode, finding that it can be improved to $691 \mathrm{~nm} / \mathrm{RIU}$ by broadening the ring width thereby increasing the field enhancement in the sensing region.

An experimental and theoretical study of sensitivity of high aspect ratio nanostructures has been reported in [14], revealing a strong dependence of the bulk sensitivity on the nanostructure height and gap width. Based on the results, the increase in height produces a larger optical volume at the resonance wavelength, easier detection of the absolute wavelength shift produced by changes in the local dielectric environment, and more bulk sensitivity up to $598 \mathrm{~nm} / \mathrm{RIU}$. Similar results and conclusions are reported in [15], and additionally that silver nanorods can offer up to 2Í more sensitivity than gold nanorods. Also, increasing the width of nanorods increases the bulk sensitivity for both metals. The sensitivity of silver nanorods reaches values $>400 \mathrm{~nm} / \mathrm{RIU}$ while that of gold nanorods is $\sim 250 \mathrm{~nm} / \mathrm{RIU}$. Bulk sensitivity measurements in [16] reveal that large nanostars show higher sensitivity than nanoparticles with other morphologies. Shen et al. presented an optimization approach to improve the sensitivity of plasmonic nanostructures by maximizing their surface enhanced Raman scattering (SERS) [17]. The bulk sensitivity of patterned Au spherical nanoparticle arrays on large-area glass substrates was measured as 43.4, 54.4 and $85.0 \mathrm{~nm} / \mathrm{RIU}$ for 10 , 25.7 and $33 \mathrm{~nm}$ high nanoparticles over the index range from 1.3284 to 1.5514 [18]. 


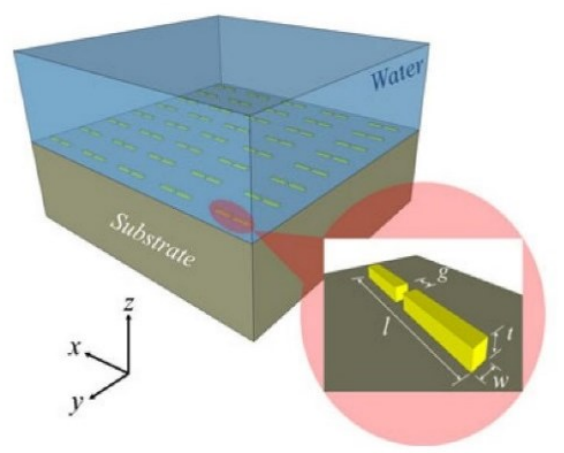

Fig. 1. Array of Au nanodipoles on silicon or glass covered by water; a plane wave source illuminates the array in the $z$-direction from the top or bottom.

While much of the literature is concentrated on investigating nanofeature geometry to enhance the bulk sensitivity, there is one report on the general analytical estimation of LSP sensitivity regardless of geometry by Zalyubovskiy et al. [19], who showed that the sensitivity is directly proportional to the resonance wavelength and that it depends on the fraction of the electromagnetic fields confined within the sensing volume (which is always less than unity). Using a quasistatic theory, they found an upper bound to the sensitivity of nanoparticles on dielectric substrates such as those associated with bimolecular sensing.

Although many theoretical and experimental studies have been carried out on various aspects of nanoparticles and nanoantennas and their applications, a systematic study of the bulk sensitivity of nanodipole antennas to variations in design is still needed. In this paper, we present a full study of the spectral response of an infinite array of rectangular gold nanodipoles on a substrate (silicon or glass) covered by water; these substrates are chosen because they are inexpensive and broadly available. The main goal of the paper is to find a set of optimized dimensions for designing an array of nanodipoles which provides maximum bulk sensitivity at three wavelengths of interest, $\lambda_{0 d}$ $=850 \mathrm{~nm}, \lambda_{0 d}=1310 \mathrm{~nm}$ and $\lambda_{0 d}=1550 \mathrm{~nm}$ (subscripts "0" and " $d$ " stand for "free-space" and "desired", respectively). These wavelengths are justified by the availability of inexpensive and compact laser sources. All calculations rely on modal analysis and a transmission line model proposed and validated in [4], [21].

In section II the problem geometry is described; in section III we summarize the design equation for an array of nanodipoles, we give a simple formula for the bulk sensitivity based on modal parameters, and we describe our optimization strategy; in section IV results are shown for six test cases determined by the wavelength and substrate of interest, trends are identified and the best designs summarized; finally in section $\mathrm{V}$ we validate some results of section IV via 3D numerical solution (FDTD); concluding remarks are then given in section VI.

\section{GEOMETRY}

Fig. 1 shows the biosensor concept, consisting of an array of Au nanodipole antennas on a substrate. The array is symmetric about the $x$ and $y$ axes. It is assumed illuminated by an

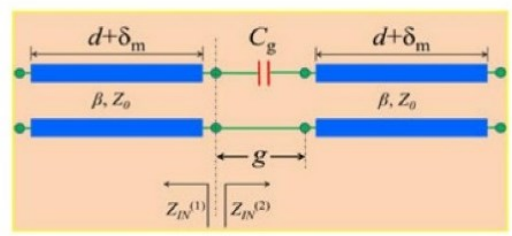

Fig. 2. Transmission line model of a dipole antenna where the gap is modeled as a parallel-plate capacitor [21].

$x$-polarised plane wave directed onto the array near the resonance wavelength from the top or through the substrate (except for silicon at $\lambda_{0 d}=850 \mathrm{~nm}$ which is not transparent at this wavelength). In a biosensing application, bottom illumination may be advantageous as it separates the optics (bottom) from the micro-fluidics (top) [4]. A single nanodipole with relevant dimensions is depicted in Fig. 1.

\section{NANODIPOLE DESIGN AND BULK SENSITIVITY}

One of the important objectives in optical antenna design for sensing is to find the optimum geometry of an antenna to operate at a specific resonance wavelength with a large bulk sensitivity. In this section, we summarise a simple method to design nanodipoles, and optimize their bulk sensitivity (the underlying theory originates from [4], [21]).

\section{A. Nanodipole Design}

Given a desired resonance frequency, $\omega_{0 d}$ (or wavelength $\lambda_{0 d}$ ), we seek the dimensions of a nanodipole. Fig. 2 shows an equivalent circuit for the antenna under perpendicular $x$-polarised illumination, as capacitor-coupled open-circuited transmission lines, where the capacitor models the gap between the dipole arms. The model relates $\omega_{0 d}$ to the effective refractive index $\left(n_{\text {eff }}\right)$ of the $s a_{b}^{0}$ mode [20] excited along the nanowires forming the nanodipole arms, and to the nanodipole gap, as follows [21]:

$$
\tan \left(n_{\mathrm{eff}} \omega_{0 d}\left(d+\delta_{m}\right) \sqrt{\varepsilon_{0} \mu_{0}}\right)=-2 \omega_{0 d} C_{g} Z_{0}
$$

where

$$
\omega_{0 d}=\frac{2 \pi c_{0}}{\lambda_{0 d}}
$$

and $c_{0}$ is the speed of light in the vacuum. The parameter $\delta_{m}$ is the distance from the end of each arm where the main field component decays to $1 / e$ and the term $\left(d+\delta_{m}\right)$ is the effective length of each transmission line section. We ignore the effect of $\delta_{m}$ in this paper, so $l=2 d+g$. Neglecting fringing electric fields, the capacitance of the gap, $C_{g}$, is taken as

$$
C_{g}=\varepsilon_{0} \varepsilon_{\text {water }} \frac{w \cdot t}{g}
$$

and $\varepsilon_{\text {water }}$ is the relative permittivity of water filling the gap at the wavelength of modal analysis. Also $Z_{0}$ is the characteristic impedance of the transmission lines, taken numerically as the weighted average wave impedance of the $s a_{b}^{0}$ mode, following [20]. 
Our design procedure starts by assigning initial dimensions to $w, t$ and $g$, and solving (1) for $d$, providing a good estimate for the length via

$$
d=\frac{1}{n_{\mathrm{eff}} \omega_{0 d} \sqrt{\varepsilon_{0} \mu_{0}}}\left(\pi-\tan ^{-1}\left(2 \omega_{0 d} C_{g} Z_{0}\right)\right)
$$

where the function $\tan ^{-1}$ returns the angle located in the first region of the trigonometric circle, i.e.

$$
0<\tan ^{-1}\left(2 \omega_{0 d} C_{g} Z_{0}\right)<\frac{\pi}{2} .
$$

For instance, for a nanodipole on glass resonating at $\lambda_{0 d}=$ $1310 \mathrm{~nm}$, one may choose $w=10 \mathrm{~nm}$ and $t=20 \mathrm{~nm}$, consequently the modal parameters (of the $s a_{b}^{0}$ mode) are calculated as $n_{\mathrm{eff}} \approx 4.5, Z_{0} \approx 860 \Omega$, then setting the gap to $g=20 \mathrm{~nm}$ yields $C_{g} \approx 0.15 \mathrm{aF}$; substituting these values in (3), yields the length of the nanodipole antenna as about $l \approx 290 \mathrm{~nm}$.

\section{B. Bulk Sensitivity Calculations}

Spectral interrogation is assumed for measuring changes in the bulk refractive index of the cover medium (water) by monitoring changes in the resonance wavelength of the nanodipoles. The bulk sensitivity $S_{B}$ can be expressed as [4]:

$$
S_{B}=S_{n}+S_{C_{g}}+S_{Z}
$$

where $S_{n}, S C_{g}$, and $S_{z}$ denote the sensitivity of the effective refractive index, the gap capacitance, and the characteristic impedance, respectively:

$$
\left\{\begin{array}{l}
S_{B}=\frac{\partial \lambda_{0 d}}{\partial n_{B}} \\
S_{n}=\left(-\frac{\lambda_{0 d}^{2}}{2 \pi c_{0}}\right) \zeta_{\omega}^{-1} \cdot \zeta_{n} \frac{\partial n_{\mathrm{eff}}}{\partial n_{B}} \\
S_{C_{g}}=\left(-\frac{\lambda_{0 d}^{2}}{2 \pi c_{0}}\right) \zeta_{\omega}^{-1} \cdot \zeta_{C} \frac{\partial C_{g}}{\partial n_{B}} \\
S_{Z}=\left(-\frac{\lambda_{0 d}^{2}}{2 \pi c_{0}}\right) \zeta_{\omega}^{-1} \cdot \zeta_{Z} \frac{\partial Z_{0}}{\partial n_{B}}
\end{array}\right.
$$

and the coefficients are defined as follows:

$$
\left\{\begin{array}{l}
\zeta_{\omega}=d c_{0}^{-1} n_{\mathrm{eff}}\left(1+4 \omega_{0 d}^{2} C_{g}^{2} Z_{0}^{2}\right)+2 C_{g} Z_{0} \\
\zeta_{n}=d c_{0}^{-1} \omega_{0 d}\left(1+4 \omega_{0 d}^{2} C_{g}^{2} Z_{0}^{2}\right) \\
\zeta_{C}=-2 \omega_{0 d} Z_{0} \\
\zeta_{Z}=-2 \omega_{0 d} C_{g}
\end{array}\right.
$$

where $n_{B}$ stands for the refractive index of water. In addition to maximizing $S_{B}$, we are interested in investigating the relative contributions of the terms on the right hand side of (4). It is shown in [4] that nanodipoles provide greater bulk sensitivity than monopoles because of the existence of the gap in the former. The nanodipoles collect light over an effective area, concentrating its energy to the gap region. The term $S_{C g}$ takes into account the gap effects and is expected to contribute importantly to $S_{B}$.

\section{Optimization Process}

It can be easily seen from (4) to (6) that the bulk sensitivity $S_{B}$ is a function of the geometry of the nanodipole and of the refractive index of the media involved (substrate, metal, water); i.e. $n_{\text {eff }}=n_{\text {eff }}\left(n_{B}, n_{S}, n_{M}, w, t\right), \omega_{0 r}=\omega_{0 r}\left(n_{B}, n_{S}, n_{M}\right.$, $w, t, l), C_{g}=C_{g}\left(n_{B}, w, t\right)$, and $Z_{0}=Z_{0}\left(n_{B}, n_{S}, n_{M}, w, t\right)$ where the subscripts $B, S$, and $M$ represent bulk, substrate, and metal, respectively. It is thus appropriate to investigate dependences by summarizing the computations graphically. We propose an optimization algorithm to investigate the array performance for two substrates, Silicon $(\mathrm{Si})$ and Glass $\left(\mathrm{SiO}_{2}\right)$, assuming Au nanodipoles, using wavelength-dependent refractive indices from [22] for these materials at the three wavelengths of interest, $\lambda_{0 d}=850 \mathrm{~nm}, \lambda_{0 d}=1310 \mathrm{~nm}$ and $\lambda_{0 d}=1550 \mathrm{~nm}$. The refractive index of water was taken as $n_{B}=1.329,1.32235$ and 1.318 at $\lambda_{0 d}=850 \mathrm{~nm}, \lambda_{0 d}=1310 \mathrm{~nm}$ and $\lambda_{0 d}=1550 \mathrm{~nm}$, respectively [23]. The algorithm consists of the following four steps.

1) Design the nanodipole antenna to resonate at a desired wavelength using (3).

2) Change the background refractive index (water) by perturbing its real part by $h_{B}= \pm 10^{-3}$ for the design of step $\mathrm{i}$, then find two new corresponding $n_{\text {eff }}$ and $Z_{0}$, and calculate the bulk sensitivity using (4) to (6). The terms $\partial n_{\text {eff }} / \partial n_{B}$, $\partial C_{g} / \partial n_{B}$ and $\partial Z_{0} / \partial n_{B}$ are approximated via an $\mathrm{O}\left(h^{2}\right)$ central finite-difference formula as follows:

$$
\frac{\partial f}{\partial n_{B}}=\frac{f\left(n_{B}+h_{B}\right)-f\left(n_{B}-h_{B}\right)}{2 h_{B}}
$$

where $f$ represents $n_{\mathrm{eff}}, C_{g}$, and $Z_{0}$.

3) Change the thickness of the nanodipole and repeat step ii, keeping all other dimensions fixed, in order to determine the effect of the thickness on the bulk sensitivity. Repeat many times using a small change in thickness in order to gather a full data set.

4) Change the width of the nanodipole and repeat steps ii and iii, keeping all other dimensions fixed, in order to understand the effect of width on the bulk sensitivity. Repeat many times using a small change in width in order to gather a full data set.

Changes in the bulk sensitivity with respect to changes in the gap length are determined straightforwardly using the modal results acquired in the previous steps and (2), (3), (5), and (6). Optimal designs are identified by locating the maximum bulk sensitivity.

\section{RESUlTS AND DiscusSiON}

In this section we investigate systematically the bulk sensitivity and its dependence on the design parameters and we summarize the best designs for each test case. For each test case (e.g. $\lambda_{0 d}=1310 \mathrm{~nm}$ on $\mathrm{Si}$ ), we calculate the bulk sensitivity and observe trends versus $t$ and $w$, then we pick a thickness (e.g. $t$ $=35 \mathrm{~nm}$ ) and follow trends versus $g$ and $w$.

\section{A. Silicon Substrate at $\lambda_{0 d}=850 \mathrm{~nm}$}

The first substrate chosen is silicon and the resonance wavelength is $\lambda_{0 d}=850 \mathrm{~nm}$. Fig. 3 shows the modal parameters and bulk sensitivity swept over thickness, width and gap. The results reveal that the characteristic impedance $Z_{0}$ drops significantly as the thickness increases. Also, for a specific thickness, decreasing the width increases the characteristic impedance. The derivative $\partial Z_{0} / \partial n_{B}$ was also computed and found to be 

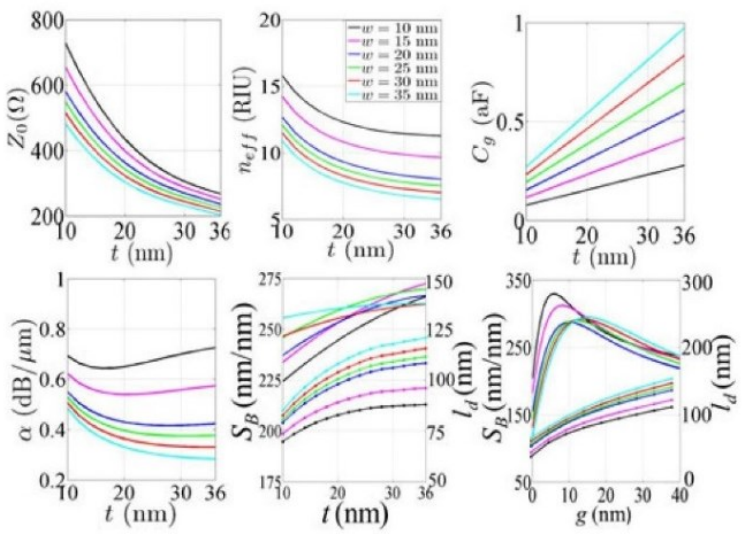

Fig. 3. Modal parameters, bulk sensitivity, and length for an array of nanodipoles covered by water on silicon designed for $\lambda_{0 d}=850 \mathrm{~nm}$ versus thickness (or gap), swept over width with $g=20 \mathrm{~nm}$, pitch size of $300 \mathrm{~nm} \times 300 \mathrm{~nm}$; top: (left) characteristic impedance, (middle) effective refractive index, (right) capacitance of the gap, bottom: (left) mode power attenuation, (middle) bulk sensitivity and nanodipole length, (right) bulk sensitivity and nanodipole length swept over $g(t=35 \mathrm{~nm})$.

negative, so it reduces $S_{B}$ in (4). The effective refractive index $n_{\text {eff }}$ exhibits the same behavior as $Z_{0}$ but its derivative $\partial n_{\text {eff }} / \partial n_{B}$ is positive, and thus increases the bulk sensitivity in (4). Finally the last parameter, $C_{g}$, increases strongly with thickness (and width) due to the increasing cross-sectional area $(w t)$ of the nanodipole arms. The derivative $\partial C_{g} / \partial n_{B}$ is positive and significantly increases the bulk sensitivity.

The mode power attenuation $\alpha$ is also shown in Fig. 3 as a function of $w$ and $t$. No obvious trend with thickness is observed for the cases $w=10,20 \mathrm{~nm}$, but for the other cases the attenuation decreases with increasing thickness. Also, the attenuation decreases with increasing widths for all thicknesses. This is due to the fact that the fields of the mode penetrate deeper into the $\mathrm{Au}$ as its thickness or width decrease.

The bulk sensitivity $S_{B}$ is plotted versus thickness in the bottom-middle panel and its values are labeled on the left axis. The right axis shows the corresponding nanodipole length (curves with dots). Increasing the nanodipole aspect ratio (i.e., making taller antennas) increases the bulk sensitivity. This trend is in agreement with an electrostatic approximation based on Mie theory [10]. We also recall the existence of a theoretical upper limit for LSP bulk sensitivity, which does not depend on the shape of the particle [19]. To check the validity of this fact, we increased the thickness for the case $w=10 \mathrm{~nm}$ to $t=80 \mathrm{~nm}$ and calculated $S_{B} \approx 284 \mathrm{~nm} / \mathrm{RIU}$ indicating saturation of the bulk sensitivity.

It is worth understanding "how much" bulk sensitivity comes from each term in (4). To this end, we consider one width $w$ $=20 \mathrm{~nm}$ and calculate the ratios $\left|S_{C g} / S_{Z}\right|$ and $S_{C g} / S_{n}$ as a function of thickness. Increasing the thickness increases $S_{C g}$, so we expect an increasing trend for both of these ratios. The ratio $\left|S_{C g} / S_{Z}\right|$ increases from $30(t=10 \mathrm{~nm})$ to $2370(t=$ $36 \mathrm{~nm}$ ), revealing the dominance of the gap capacitance term over the characteristic impedance term. Also the ratio $S_{C g} / S_{n}$ varies from $0.6(t=10 \mathrm{~nm})$ to $1(t=36 \mathrm{~nm})$ indicating that both terms are of comparable importance.
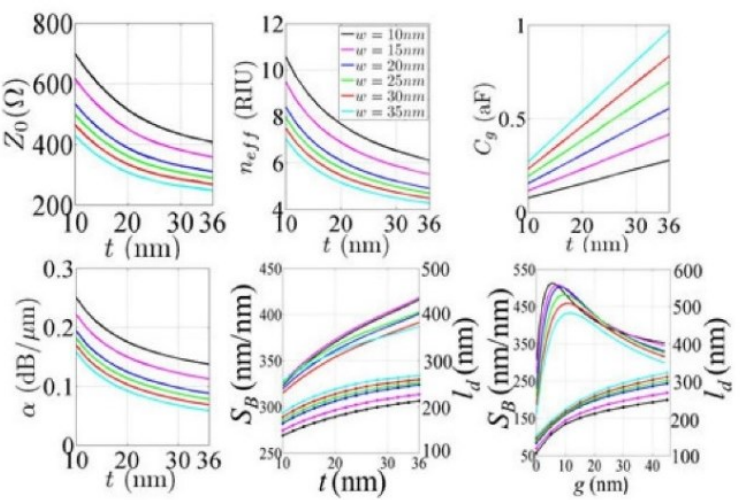

Fig. 4. Modal parameters, bulk sensitivity, and length for an array of nanodipoles covered by water on silicon designed for working at $\lambda_{0 d}=1310 \mathrm{~nm}$ versus thickness (or gap) swept over width with $g=20 \mathrm{~nm}$, pitch size of $300 \mathrm{~nm} \times 300 \mathrm{~nm}$; top: (left) characteristic impedance, (middle) effective refractive index, (right) capacitance related to the gap region, bottom: (left) mode power attenuation, (middle) bulk sensitivity and nanodipole length, (right) bulk sensitivity and nanodipole length swept over $g(t=35 \mathrm{~nm})$.

The bulk sensitivity is plotted versus gap length for $t=35 \mathrm{~nm}$ in the bottom-right panel and its values are labeled on the left axis. The right axis shows the corresponding nanodipole length (curves with dots). We note from these curves the existence of maximum values for $S_{B}$ at specific gap lengths. Reducing the gap length increases the capacitance but in the limit $g \rightarrow 0, C_{g}$ $\rightarrow \infty$, and $\zeta_{\omega}, \zeta_{n}$ and $\zeta_{Z}$ become very large, consequently (4) tends to $S_{B} \rightarrow S_{n}$ and a drop in bulk sensitivity occurs. At the other extreme, $g \rightarrow \infty, C_{g} \rightarrow 0$ and in this limit $\zeta_{Z}=0$ which causes a drop in bulk sensitivity. A maximum therefore exists between these extrema.

Based on the results obtained, the best design in terms of bulk sensitivity is considered as $g=20 \mathrm{~nm}, w=25 \mathrm{~nm}$, sweeping over $t$ with the corresponding length.

\section{B. Silicon Substrate at $\lambda_{0 d}=1310 \mathrm{~nm}$}

Fig. 4 shows the modal parameters and bulk sensitivity swept over thickness, width and gap for this test case. Results reveal that characteristic impedance $Z_{0}$ drops significantly as the thickness increases. Also, for a specific thickness, decreasing the width increases the characteristic impedance. The refractive index of water is approximately the same at $\lambda_{0 d}=1310 \mathrm{~nm}$ and $\lambda_{0 d}=850 \mathrm{~nm}$, so we do not expect a significant difference in the gap capacitance.

The mode power attenuation $\alpha$ is also shown in Fig. 4 as a function of $w$ and $t$. It is smaller than that at $\lambda_{0 d}=850 \mathrm{~nm}$ so we expect narrower spectral responses [21] which is advantageous in a sensor application [24]. The attenuation decreases with increasing thickness and width.

The bulk sensitivity $S_{B}$ is plotted versus thickness in the bottom-middle panel and its values are labeled on the left axis. The right axis shows the corresponding nanodipole length (curves with dots). Increasing the nanodipole aspect ratio increases the bulk sensitivity. Values also show larger $S_{B}$ with respect to $\lambda_{0 d}=850 \mathrm{~nm}$ which comes from the larger 

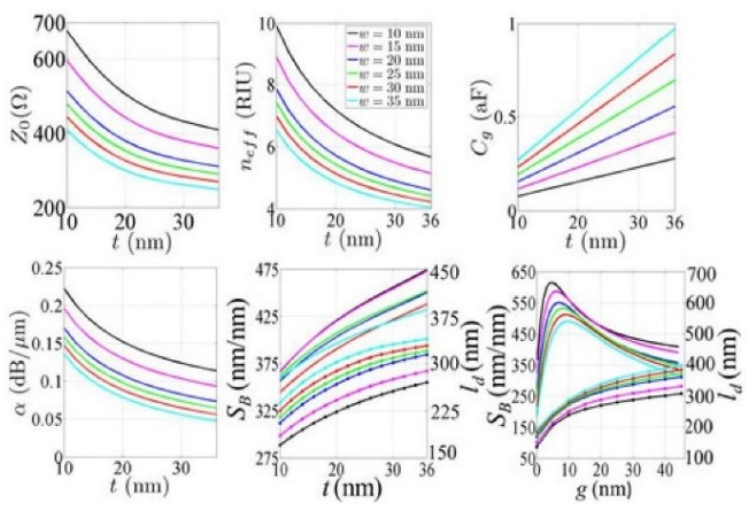

Fig. 5. Modal parameters, bulk sensitivity, and length for an array of nanodipoles covered by water on silicon designed for working at $\lambda_{0 d}=1550 \mathrm{~nm}$ versus thickness (or gap) swept over width with $g=20 \mathrm{~nm}$, pitch size of $360 \mathrm{~nm} \times 360 \mathrm{~nm}$; top: (left) characteristic impedance, (middle) effective refractive index, (right) capacitance related to the gap region, bottom: (left) mode power attenuation, (middle) bulk sensitivity and nanodipole length, (right) bulk sensitivity and nanodipole length swept over $g(t=35 \mathrm{~nm})$

coefficients in (5) at the higher wavelengths. For example, in the case $w=20 \mathrm{~nm}$, the ratio $S_{C g-(1310 \mathrm{~nm})} / S_{C g-(850 \mathrm{~nm})}$ increases from 1.27 to 1.68 which causes an increase in bulk sensitivity. It is also shown that increasing the nanodipole width $w$ decreases the bulk sensitivity. In fact by increasing $w, S_{C g}$ increases, while the values of $S_{Z}$ and $S_{n}$ decrease and $S_{B}$ drops.

The bulk sensitivity is plotted versus gap length for $t=35 \mathrm{~nm}$ in the bottom-right panel and its values are labeled on the left axis. The right axis shows the corresponding nanodipole length (curves with dots).

Based on the results obtained, the best design in terms of bulk sensitivity is considered as $g=20 \mathrm{~nm}, w=10 \mathrm{~nm}$, sweeping over $t$ with the corresponding length.

\section{Silicon Substrate at $\lambda_{0 d}=1550 \mathrm{~nm}$}

Fig. 5 shows the modal parameters and bulk sensitivity swept over thickness, width and gap for this test case. The results reveal that the characteristic impedance $Z_{0}$ drops significantly as the thickness increases. Also, for a specific thickness, decreasing the width increases the characteristic impedance. The gap capacitance is approximately the same as in the previous cases as the refractive index of water is approximately same.

The mode power attenuation is also shown in Fig. 5 as a function of $w$ and $t$. It is again smaller than ones predicted at $\lambda_{0 d}=850 \mathrm{~nm}$ and $\lambda_{0 d}=1310 \mathrm{~nm}$. The mode power attenuation decreases with increasing thickness and widths.

The bulk sensitivity $S_{B}$ is plotted versus thickness in the bottom-middle panel and its values are labeled on the left axis. The right axis shows the corresponding nanodipole length (curves with dots). Increasing the nanodipole aspect ratio increases the bulk sensitivity. The $S_{B}$ values are larger than in the previous test cases due to an increase in the coefficients in (5). For example, in the case $w=20 \mathrm{~nm}$, the ratios $S_{C g-(1550 \mathrm{~nm})}$ $/ S_{C g-(850 \mathrm{~nm})}$ and $S_{C g-(1550 \mathrm{~nm})} / S_{C g-(1310 \mathrm{~nm})}$ increase from 1.68 to 1.71 and from 1.02 to 2.2 , respectively. It is also shown
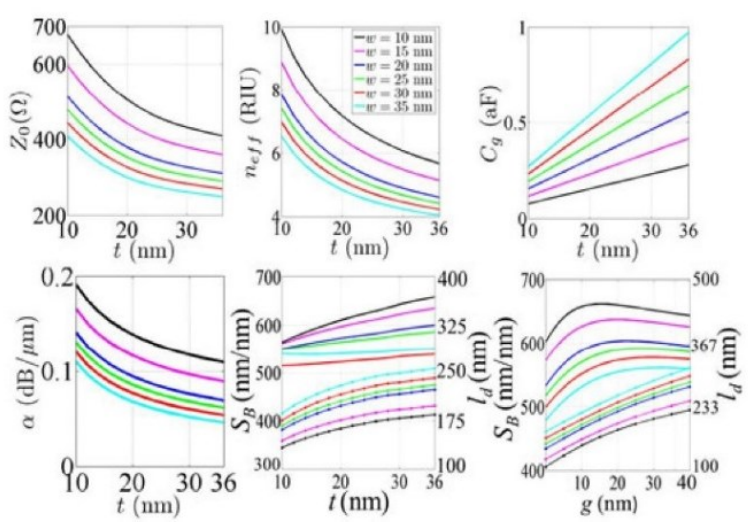

Fig. 6. Modal parameters, bulk sensitivity, and length for an array of nanodipoles covered by water on glass designed for working at $\lambda_{0 d}=850 \mathrm{~nm}$ versus thickness (or gap) swept over width with $g=20 \mathrm{~nm}$, pitch size of $300 \mathrm{~nm} \times 300 \mathrm{~nm}$; top: (left) characteristic impedance, (middle) effective refractive index, (right) capacitance related to the gap region, bottom: (left) mode power attenuation, (middle) bulk sensitivity and nanodipole length, (right) bulk sensitivity and nanodipole length swept over $g(t=35 \mathrm{~nm})$.

that increasing the nanodipole width $w$ decreases the bulk sensitivity. In fact by increasing $w, S_{C g}$ increases, while the values of $S_{Z}$ and $S_{n}$ decrease and $S_{B}$ drops.

The bulk sensitivity is plotted versus gap length for $t=35 \mathrm{~nm}$ in the bottom-right panel and its values are labeled on the left axis. The right axis shows the corresponding nanodipole length (curves with dots).

Based on the results obtained, the best design in terms of bulk sensitivity is considered as $g=20 \mathrm{~nm}, w=10 \mathrm{~nm}$, sweeping over $t$ with the corresponding length.

\section{Glass Substrate at $\lambda_{0 d}=850 \mathrm{~nm}$}

The substrate in this section is changed to glass and the resonance wavelength is set to $\lambda_{0 d}=850 \mathrm{~nm}$. Fig. 6 shows the modal parameters and bulk sensitivity swept over thickness, width and gap. Comparing to the results of section $\mathrm{A}$, the mode power attenuation $\alpha$ is much smaller which will lead to narrower resonance responses. The bulk sensitivity is also larger than the ones predicted in section A.

The reason for this can be found by observing the electric field of the mode near the nanodipoles. Fig. 7 shows the real part of the main transverse electric field $\left(E_{z}\right)$ of the mode, plotted over the $y z$ cross-section of one of the dipole arms for $w$ $=20 \mathrm{~nm}$ and $t=20 \mathrm{~nm}$ on glass and silicon. The distribution of $E_{z}$ identifies the mode propagating along the nanodipole arms as the $s a^{0}{ }_{b}$ mode, which is excited on the nanodipoles given the polarization, orientation and uniformity of the source field ( $x$-polarized plane wave). As can be seen the electric field of the mode extends more within the sensing region for the case of the glass substrate, and consequently is more susceptible to perturbations (thus the greater bulk sensitivity). Thus, reducing the refractive index of the substrate (silicon to glass) produces a structure that is more symmetric when covered by water, which in turn allows the fields to become more symmetric, enhancing the interaction with the sensing region. 


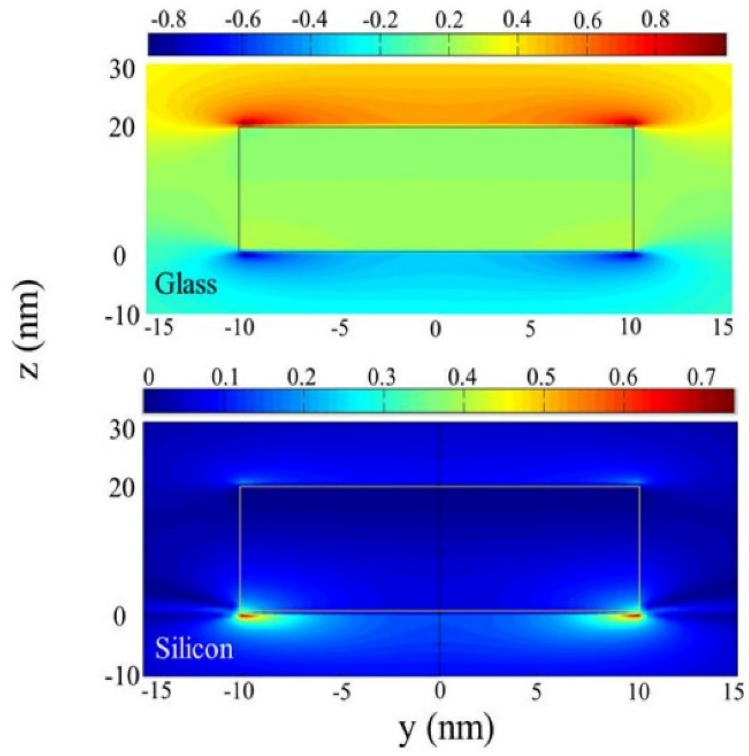

Fig. 7. Real part of $E_{z}$ of the $s a^{0}{ }_{b}$ mode plotted over the cross-section of a nanodipole arm $\left(\lambda_{0 d}=850 \mathrm{~nm}\right)$ computed using a mode solver; (top) glass and (bottom) silicon.
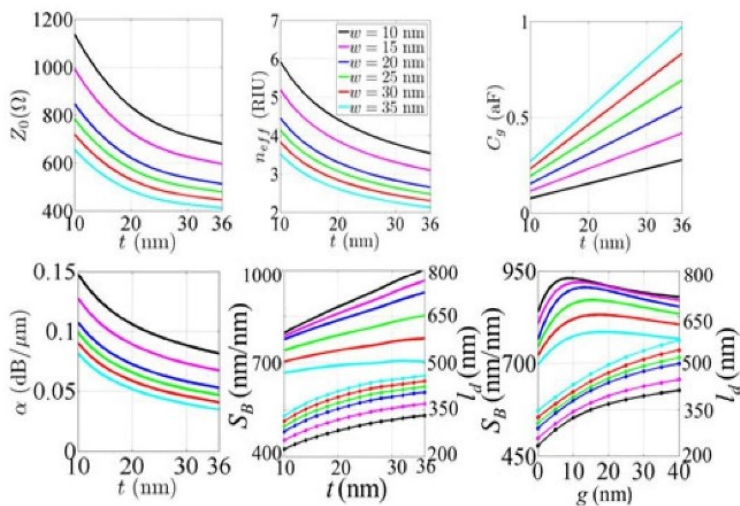

Fig. 8. Modal parameters, bulk sensitivity, and length for an array of nanodipoles covered by water on glass designed for working at $\lambda_{0 d}=1310 \mathrm{~nm}$ versus thickness (or gap) swept over width with $g=20 \mathrm{~nm}$, pitch size of $440 \mathrm{~nm} \times 440 \mathrm{~nm}$; top: (left) characteristic impedance, (middle) effective refractive index, (right) capacitance related to the gap region, bottom: (left) mode power attenuation, (middle) bulk sensitivity and nanodipole length, (right) bulk sensitivity and nanodipole length swept over $g(t=35 \mathrm{~nm})$.

Based on the results obtained, the best design in terms of bulk sensitivity is considered as $g=20 \mathrm{~nm}, w=10 \mathrm{~nm}$, sweeping over $t$ with the corresponding length.

\section{E. Glass Substrate at $\lambda_{0 d}=1310 \mathrm{~nm}$}

Fig. 8 shows the modal parameters and bulk sensitivity swept over thickness, width and gap for this test case. Comparing to the results of section $\mathrm{B}$, the bulk sensitivity is larger and the mode power attenuation is smaller for reasons that are discussed in the previous section. A lower mode power attenuation narrows the resonance which in turn makes shifts in resonance easier to track. For example, the full-width at half maximum (FWHM) is
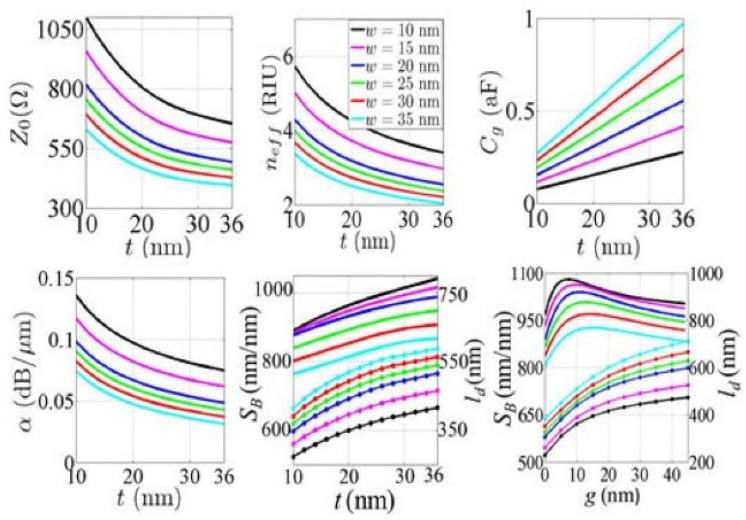

Fig. 9. Modal parameters, bulk sensitivity, and length for an array of nanodipoles covered by water on glass designed for working at $\lambda_{0 d}=1550 \mathrm{~nm}$ versus thickness (or gap) swept over width with $g=20 \mathrm{~nm}$, pitch size of $480 \mathrm{~nm} \times 480 \mathrm{~nm}$; top: (left) characteristic impedance, (middle) effective refractive index, (right) capacitance related to the gap region, bottom: (left) mode power attenuation, (middle) bulk sensitivity and nanodipole length, (right) bulk sensitivity and nanodipole length swept over $g(t=35 \mathrm{~nm})$.

calculated for this case to be $61 \mathrm{~nm}$, and for the corresponding case in section B as $488 \mathrm{~nm}$. Values also show a larger $S_{B}$ compared to $\lambda_{0 d}=850 \mathrm{~nm}$ (section A) which comes from the larger coefficients in (5) at larger wavelengths.

Based on the results obtained, the best design in terms of bulk sensitivity is considered as $g=20 \mathrm{~nm}, w=10 \mathrm{~nm}$, sweeping over $t$ with the corresponding length.

\section{F. Glass Substrate at $\lambda_{0 d}=1550 \mathrm{~nm}$}

The results for this test case are shown in Fig. 9 which reveals larger values for $Z_{0}$, and smaller values for $n_{\text {eff }}$ and the mode power attenuation compared to the previous cases. Increasing the wavelength clearly decreases the mode power attenuation. The bulk sensitivity is also larger than in all previous cases.

Based on the results obtained, the best design in terms of bulk sensitivity is considered as $g=20 \mathrm{~nm}, w=10 \mathrm{~nm}$, sweeping over $t$ with the corresponding length.

\section{Verification of Modal Results Using FDTD}

In this section we choose the best design for each test case and verify the results obtained through the transmission-line model by comparing with numerical results obtained using the FDTD method [25]. We calculate the bulk sensitivity directly by approximating the differential via an $\mathcal{O}\left(h^{2}\right)$ central finitedifference formula [26]

$$
\frac{\partial \lambda_{0 d}}{\partial n_{B}}=\frac{\lambda_{0 d}\left(n_{B}+h_{B}\right)-\lambda_{0 d}\left(n_{B}-h_{B}\right)}{2 h_{B}} .
$$

This approximation improves in accuracy as $h_{B} \rightarrow 0$ (to within machine arithmetic accuracy). In the computations, we used $h_{B}$ $=10^{-3}<<n_{B}$. Calculating this differential equation using the FDTD method is a very time-consuming process, especially for our chosen mesh of $0.5 \times 0.5 \times 0.5 \mathrm{~nm}^{3}$ for gridding the problem volume (thus highlighting the value of the transmission-line model). The parameter $\lambda_{0 d}$ is the resonance wavelength taken 


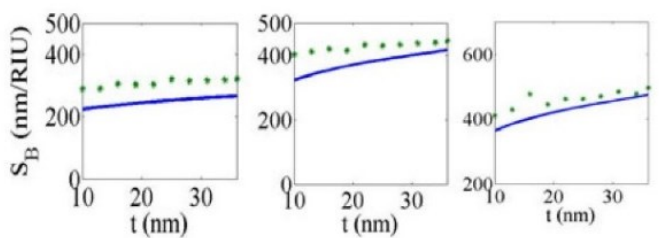

Fig. 10. Comparison of bulk sensitivity obtained from modal analysis (solic blue) to FDTD (green stars) on silicon: (left) $w=25 \mathrm{~nm}, \lambda_{0 d}=850 \mathrm{~nm}$, (middle) $w=10 \mathrm{~nm}, \lambda_{0 d}=1310 \mathrm{~nm}$, and (right) $w=10 \mathrm{~nm}, \lambda_{0 d}=1550 \mathrm{~nm}$

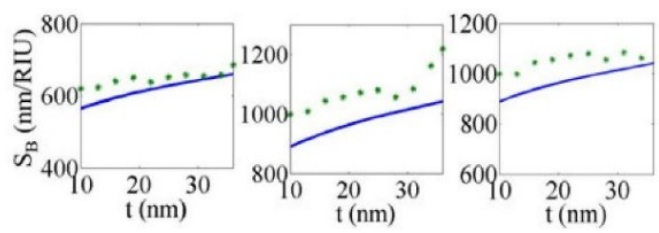

Fig. 11. Comparison of bulk sensitivity obtained from modal analysis (solid blue) to FDTD (green stars) on glass: (left) $w=10 \mathrm{~nm}, \lambda_{0 d}=850 \mathrm{~nm}$, (middle) $w=10 \mathrm{~nm}, \lambda_{0 d}=1310 \mathrm{~nm}$, and (right) $w=10 \mathrm{~nm}, \lambda_{0 d}=1550 \mathrm{~nm}$.

from the absorptance spectra for each design. The absorptance spectra are calculated using the transmittance and reflectance spectra, as in [21].

Transmittance and reflectance monitors were placed $2.5 \mu \mathrm{m}$ below and above the substrate $/ \mathrm{H}_{2} \mathrm{O}$ interface, respectively, parallel to the $x y$ plane. An $x$-polarized plane wave source located $2.8 \mu \mathrm{m}$ above the interface illuminates the array along the $-z$ direction.

It is worth to say that in simulations of the best designs of sections $\mathrm{C}, \mathrm{E}$, and $\mathrm{F}$, the designed lengths of nanodipoles exceed $300 \mathrm{~nm}$ which is the pitch size, so we changed the pitch size to $360 \mathrm{~nm} \times 360 \mathrm{~nm}, 440 \mathrm{~nm} \times 440 \mathrm{~nm}$ and $480 \mathrm{~nm} \times$ $480 \mathrm{~nm}$, respectively, to do FDTD calculations. Changing the pitch dimensions does not affect the bulk sensitivity value and only changes the resonant peak location and also the shape of the absorptance curve [21].

Fig. 10 showsthree cases on silicon at the three resonant wavelengths of interest. For each wavelength, the index of water was changed from the nominal value using perturbation method and absorptance spectra as described above. $S_{B}$ is calculated using (7) by monitoring the change in the resonance wavelength. As can be seen, there is a good agreement between the results.

\section{CONCLUSION}

We developed, presented, and discussed the optimization of arrays of nanodipoles for use as a (bio)sensor. It was demonstrated theoretically and numerically that the chosen resonant wavelength, substrate permittivity and the dimensions of the nanodipoles are important parameters in the design and optimisation of the structure to maximise the bulk sensitivity. Particularly, it was shown that increasing the thickness and width of nanodipoles can increase the bulk sensitivity, whereas the gap length exhibits an optimum where the sensitivity is largest. Reducing the substrate permittivity (e.g., from silicon to glass) changes the SPP mode fields on the nanodipoles such that they overlap more with the sensing region; consequently nanodipoles on glass provide more bulk sensitivity than on $\mathrm{Si}$ at the same wavelength. Operating at larger resonant wavelengths also increases the bulk sensitivity. The largest bulk sensitivities predicted are $1000 \mathrm{~nm} / \mathrm{RIU}$ on glass and $500 \mathrm{~nm} / \mathrm{RIU}$ on silicon, both at $\lambda_{0 d}=1550 \mathrm{~nm}$, and are very promising in contrast to the values reported in the literature as summarized in the introduction. The calculations were carried out using simple formulae relying on modal analysis, and some trends and results were verified using full-wave FDTD computations.

Fig. 11 shows the results of the three test cases on glass at the same three resonant wavelengths. These results are also in good agreement with each other.

\section{REFERENCES}

[1] J.Homola, Surface Plasmon Resonance Based Sensor, vol. 4. New York, NY, USA: Springer, 2006.

[2] F. J. Rodríguez, M. Martínez, B. Tomás, R. Ortuño, J. Martí, A. Martínez, and P. J. Rodríguez, "Highly-sensitive chemical detection in the infrared regime using plasmonic gold nanocrosses," Appl. Phys. Lett., vol. 98, no. 13, pp. 133118-21, Mar. 2011.

[3] C.-Y. Tsai, S. P. Lu, J. W. Lin, and P. T. Lee, "High sensitivity plasmonic index sensor using slablike gold nanoring arrays," Appl. Phys. Lett., vol. 98, no. 15, pp. 153108-1-153108-11, Apr. 2011.

[4] M. Alavirad, S. Siadat Mousavi, L. Roy, and P. Berini, "Schottky-contact plasmonic dipole rectenna concept for biosensing," Opt. Exp., vol. 21, no. 4 , pp. $4328-4347$, Feb.

[5] R. Adato and H. Altug, "In-Suit ultra-sensitive infrared absorption spectroscopy of biomolecule interactions in real time with plasmonic nanoantennas," Nat. Comm., vol. 4, 2013.

[6] M. E. Stewart, C. R. Anderton, L. B. Thompson, J. Maria, S. K. Gray, J. A. Rogers, and R. G. Nuzzo, "Nanostructured plasmonic sensors," Chem. Rev., vol. 108, no. 2, pp. 494-521, Jan., 2008.

[7] M. M. Miller and A. A. lazarides, "Sensitivity of metal nanoparticle surface plasmon resonance to the dielectric environment," J. Phys. Chem. B, vol. 109, no. 46, pp. 21556-21565, Oct. 2005.

[8] H. X. Xu and M. Kall, "Modeling the optical response of nanoparticlebased surface plasmon resonance," Sens. Actuators B, vol. 87, no. 2, pp. 244-247, Dec. 2002.

[9] J. Homola, S. S. Yee, and G. Gauglitz, "Surface plasmon resonance sensors: review," Sens. Actuators B-Chem., vol. 54, no. 1-2, pp. 3-15, Jan. 1999.

[10] P. Kvasnicka and J. Homola, "Optical sensor based on spectroscopy of localized surface plasmons on metallic nanoparticles: Sensitivity considerations," Biointerphases, vol. 3, no. 3, pp. FD4-FD11, Sep. 2008.

[11] H. Chen, X. Kou, Z. Yang, W. Ni, and J. Wang, "Shape- and size-dependent refractive index sensitivity of gold nanoparticles," Langmuir, vol. 24, no. 10 , pp. 5233-5237, Apr. 2008.

[12] Z. Chen, C. Wang, L. Wang, C. Jiang, and H. Zhu, "Surface plasmon resonance sensor by metal strip pair arrays," Opt. Quant.Electron., vol. 45, no. 7, pp. 707-712, Jul. 2013.

[13] C. Y. Tsai, S. P. Lu, J. W. Lin, and P. T. Lee, "High sensitivity plasmonic index sensor using slablike gold nanoring arrays," Appl. Phys. Lett., vol. 98 , no. 15, pp. 153108-1-153108-11, Apr. 2011.

[14] B. Päivänranta, H. Merbold, R. Giannini, L. Büchi, S. Gorelick, C. David, J. F. Löffler, T. Feurer, and Y. Ekinci, "High aspect ratio plasmonic nanostructures for sensing applications," ACS Nano, vol. 5, no. 8, pp. 6374 6382, Jul. 2011.

[15] A. Jakab, C. Rosman, Y. Khalavka, J. Becker, A. Trügler, U. Hohenester, and C. Sönnichsen, "Highly sensitive plasmonic silver nanorods," ACS Nano, vol. 5, no. 9, pp. 6880-6885, Aug. 2011.

[16] S. Barbosa, A. Agrawal, L. R. Lorenzo, I. P. Santos, R. A. A. Puebla, A. Kornowski, H. Weller, and L. M. L. Marzan, "Tuning size and sensing properties in colloidal gold nanostars," Langmuir, vol. 26, no. 18 , pp. 14943-14950, Aug. 2010.

[17] H. Shen, N. Guillot, J. Rouxel, M. L. Chapella, and T. Toury, "Optimized plasmonic nanostructures for improved sensing activities," Opt. Exp., vol. 20, no. 19, pp. 21278-90, Sep. 2012.

[18] J. Yuan, A. Hajebifard, C. George, P. Berini, and S. Zou, "Ordered gold nanoparticle arrays on glass and their characterization," J. Colloid Interface Sci, vol. 410, pp. 1-10, Nov. 2013. 
[19] S. J. Zalyubovskiy, M. Bogdanova, A. Deinega, Y. Lozovik, A. D. Pris, K. H. An, W. P. Hall, and R. A. Potyrailo, "Theoretical limit of localized surface plasmon resonance sensitivity to local refractive index change and its comparison to conventional surface plasmon resonance sensor," JOSA A, vol. 29, no. 6, pp. 994-1002, May 2012.

[20] P. Berini, "Plasmon-polariton waves guided by thin lossy metal films of finite width: Bound modes of asymmetric structures," Phys. Rev. B, vol. 63 , no. 12 , pp. 125417-33, Mar. 2001.

[21] S. Siadat, Mousavi, P. Berini, and D. McNamara, "Periodic plasmonic nanoantennas in a piecewise homogeneous background," Opt. Exp., vol. 20, no. 16, pp. 18044-18065, Jul. 2012.

[22] E. D. Palik, Handbook of Optical Constants of Solids. San Francisco, CA, USA: Academic, 1985.

[23] D. J. Segelstein, "The complex refractive index of water," M.S. thesis, University of Missouri, Kansas City, Missouri, USA, 1981.

[24] P. Berini, "Surface plasmon photodetectors and their applications," Laser Photon. Rev., doi: 10.1002/lpor.201300019.

[25] FDTD Solutions v. 7.5.6, Lumerical Solutions Inc., Vancouver, Canada.

[26] P. Berini, "Bulk and surface sensitivities of surface plasmon waveguides," New J. of Phys., vol. 10, no. 10, pp. 1-37, Oct. 2008.

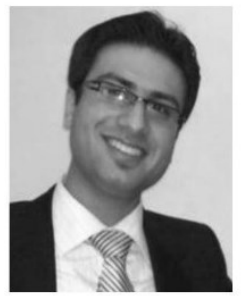

Mohammad Alavirad was born in Lahijan, Iran, in 1985. He received the B.Sc. and M.Sc. in electrical engineering from the Amirkabir University of Technology (Tehran Polytechnic) and the Sharif University of Technology, Tehran, Iran, in 2007 and 2009 , respectively. He is currently working toward the Ph.D. degree in the Department of Electronics, Carleton University, Ottawa, ON, Canada, and he is a Researcher at the School of Electrical Engineering and Computer Science, University of Ottawa, Ottawa, ON. His research interests include optical wave propagation, investigations of photonic structures, and numerical techniques in electromagnetics.

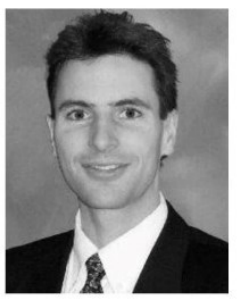

Langis Roy (M'93-A'01) received the B.A.Sc. degree in electrical engineering from the University of Waterloo, ON, Canada, in 1987, and the M.Eng. and $\mathrm{Ph}$.D. degrees from Carleton University, Ottawa, ON, Canada, in 1989 and 1993, respectively. Following a Research Fellowship with Matra Marconi Space France, in 1993, he joined the Department of Electrical Engineering, University of Ottawa as an Assistant Professor. He joined the Department of Electronics at Carleton University in 1999, where he is currently a Professor and since 2003, the Department Chair. He joined the Department of Electrical Engineering, University of Ottawa, in 1993 as an Assistant Professor. During his 2005-2006 sabbatical leave, he was a Visiting Professor at the VTT Micromodule Research Center (Oulu, Finland) and an Invited Professor at the Department of Electrical and Computer Engineering, INSA Toulouse, France. He collaborates extensively with industry and serves on the board of several research fund-granting agencies and technical conferences. His research interests include in microwave electronics, high-performance microwave circuit packaging, integrated active antennas, numerical techniques in electromagnetics, electromagnetic compatibility of ICs, optoelectronic packaging, monolithic microwave integrated circuits (MMIC), low temperature co-fired ceramics (LTCC), micro-electro-mechanical systems (MEMS), RF; millimeterwaves. Dr. Roy is a Licensed Professional Engineer in the Province of Ontario, Canada.

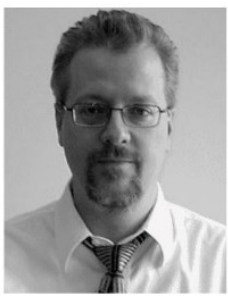

Pierre Berini (M'95-SM'06-F'11) received and the B.E.Sc. and B.Sc. degrees in electrical engineering and computer science, from the University of Western Ontario, London, ON, Canada, and the Ph.D. and M.Sc.A. degrees in electrical engineering from the École Polytechnique de Montréal, Montreal, QC, Canada, $\mathrm{He}$ is a Professor of electrical engineering, Professor of physics, and the University Research Chair in surface plasmon photonics at the University of Ottawa, and he was the Founder and Chief Technology Officer of a venture capital backed company. Dr. Berini has received an NSERC Steacie Fellowship, a Premier of Ontario Research Excellence Award (PREA), the University of Ottawa Young Researcher of the Year Award, an URSI Young Scientist Award, a George S. Glinski Award for Excellence in Research, and is a Canada Foundation for Innovation researcher. Dr. Berini is a Fellow of the Canadian Academy of Engineering, and he is a member of the OSA. He has published seven book chapters, approximately 250 scientific and technical papers in peer-reviewed periodicals and conference proceedings, and is an inventor or coinventor on 20 patents. He is an Associate (Topical) Editor of Optics Express and of Nanophotonics, and he contributes on an ongoing basis to the organization of several international conferences in photonics. His research interests include many areas of optics and photonics, with surface plasmons and their applications being of particular interest. 


\section{Chapter: Sub-Bandgap Photodetection with Electrically-Vontacted Plasmonic Nano-antennas on Silicon}

The following manuscript entitled "Photodetection with plasmonic Schottky-contact nanoantennas" is prepared to be submitted to the Optics Letters and is currently under review. The article represents work performed solely by the primary author under the supervision and guidance of the co-authors. Anthony Olivieri helped with the fabrication of nanoantennas. The characterization was performed entirely at the Berini Lab at the University of Ottawa.

The article represents the experimental results of the proposed nanoantenna. Authors propose a broadband and high-efficiency plasmonic Schottky diode using the concept of nanoantenna for detection of sub-bandgap photons in the optical communication wavelength range through internal photoemission (IPE). Using the extensive physical model on IPE (discussed in chapter 4) of hot carriers (here, holes) high values for responsivities for the proper polarization are achieved over a broad range of wavelength. Moreover, the Schottky contact area is only $500 \mu \mathrm{m}^{2}$, leading to a very small dark current $\sim 3.8 \mu \mathrm{A}$. This design is very promising for practical applications in high-density silicon photonic integration. 


\title{
Sub-bandgap photodetection with electrically- contacted plasmonic nano-antennas on silicon
}

\author{
Mohammad Alavirad, ${ }^{1,2}$ Anthony Olivieri, ${ }^{2}$ Langis Roy, ${ }^{3}$ Pierre \\ BERINI $^{2,4,5, *}$ \\ ${ }^{1}$ Department of Electronics, Carleton University, 1125 Colonel By Drive, Ottawa, ON K1S 5B6, Canada \\ ${ }^{2}$ Centre for Research in Photonics, University of Ottawa, 25 Templeton Street, Ottawa, Ontario K1N 6N5, Canada \\ ${ }^{3}$ Department of Electrical, Computer and Software Engineering, University of Ontario Institute of Technology, 2000 Simcoe Street North \\ Oshawa, Ontario L1H 7K4, Canada \\ ${ }^{4}$ School of Electrical Engineering and Computer Science, University of Ottawa, 800 King Edward Avenue, Ottawa, Ontario K1N 6N5, Canada \\ ${ }^{5}$ Department of Physics, University of Ottawa, 150 Louis Pasteur, Ottawa, Ontario K1N 6N5, Canada \\ *Corresponding author: berini@eecs.uottawa.ca
}

Received XX Month XXXX; revised XX Month, XXXX; accepted XX Month XXXX; posted XX Month XXXX (Doc. ID XXXXX); published XX Month XXXX

\begin{abstract}
We propose a Schottky contact photodetector based on electrically-contacted Au nanoantennas on p-Si for the plasmonic detection of sub-bandgap photons in the optical communications wavelength range. Based on a physical model for the internal photoemission of hot carriers, photons coupled onto the Au nanoantennas excite resonant plasmons, which decay into energetic, "hot" holes emitted over the Schottky barrier at the Au/pSi interface, resulting in a photocurrent. Moreover, the Schottky contact area is only $50 \boldsymbol{\mu m}^{2}$ and a significant portion of this contact $\left(45.5 \mu \mathrm{m}^{2}\right)$ consists of a $\mathrm{Au} / \mathrm{Ti}$ bilayer having a high Schottky barrier thus producing negligible dark current. This highly compact component is very promising for applications in high-density $\mathrm{Si}$ photonic integration. (C) 2016 Optical Society of America
\end{abstract}

OCIS codes: (220.0220) Optical design and fabrication; (230.0230) Optical devices; (240.6680) Surface plasmons; (250.5403) Plasmonics; (040.5160) Photodetectors.

http://dx.doi.org/10.1364/OL.99.099999

Plasmonic nanoantennas have become key photonic components useful for several applications involving the conversion of light from free space to ultrasmall sub-wavelength volumes. The behaviour of metals at optical frequencies creates differences between optical nanoantennas and classical microwave antennas $[1,2]$. Well-designed metallic structures having small dimensions, sharp corners and narrow gaps produce significant local field enhancement, and consequently strong enhancement of radiation emission and absorption in matter. However, large structures have better scattering-to-extinction cross-section ratios and better response in the far-field. Nanoantenna design must often trade-off these properties [3]. Nanomonopoles, nanodipoles and nanobowties are three of the most popular types of nanoantennas. In most cases, $\mathrm{Au}$ and $\mathrm{Ag}$ are used as the metal for the antennas, on a glass, ITO or Si substrate. The range of application of these types of antennas is very broad: biosensing [4, 5], photodetection [6], spectroscopy and imaging [7] and waveguiding [8] are some of the main applications.

The electric near-fields produced by nanoantennas can be of much greater intensity than the incident electric fields [9], therefore the photo-detection volume can shrink, leading potentially to faster devices with compelling signal to noise characteristics [10]. In [11] Mousavi et al predicated significant enhancement in responsivity in the presence of nanodipole antennas at telecom wavelengths. They proposed a surface plasmon-polaritons (SPP) photodetector concept, based on the enhancement of electrical near field in low-defect, low-doped $\mathrm{In}_{0.53} \mathrm{Ga}_{0.47} \mathrm{As}$ detection volumes located in the gaps of an array of metal nanodipole antennas. The 3-dB electrical bandwidth of the device was investigated based on its $R C$ rise time and the hole transit time through the detection volume for the cases of conventional and ballistic transport in InGaAs and found to range from $\sim 0.7$ to $4 \mathrm{THz}$. Nanomonopole arrays were also investigated for photodetection based on internal photoemission (IPE). The systems investigated consist of Au nanowires on $\mathrm{Si}$ covered by $\mathrm{H}_{2} \mathrm{O}$ and a thin adlayer representing a biochemical coating suggested for a biosensing application. The resonance excited on such nanoantennas by perpendicularly incident light polarised along their axis is dipolar, and comprised of an SPP waveguide mode propagating along the length of the nanowires and reflecting from the ends to form standing waves along the antenna [12] -the specific SPP waveguide mode excited on the nanowires is the $s a_{b}{ }^{0}$ mode of an asymmetric metal stripe [13].

The IPE mechanism for holes is sketched in Fig. 1. In fact, IPE is a 3-step process consisting of the photoexcitation of hot (energetic) carriers in the metal, transport with scattering of hot carriers 
towards the metal-Si interface, and the emission of hot carriers over the Schottky barrier into the semiconductor where they are collected as the photocurrent. IPE requires that the energy of the incident photons, $h v$ ( $h$ is Plank's constant and $v$ is the optical frequency), be greater than the Schottky barrier, $\Phi_{\mathrm{B}}$. This mechanism is useful for detection at energies below the semiconductor bandgap, $E_{\mathrm{g}}$.

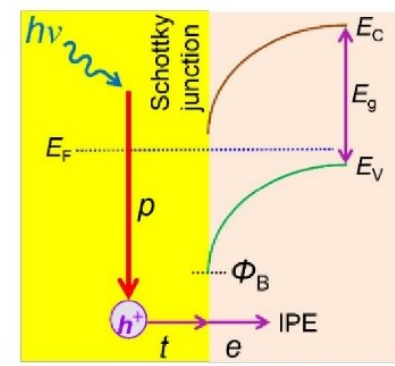

Fig. 1. Energy band diagram of a metal contact to a p-type semiconductor and the 3-steps of the internal photoemission process: $p$ : photoexcitation, $t$ : transport, $e$ : emission. $E_{\mathrm{C}}$ and $E_{\mathrm{V}}$ are the conduction and valence band edges, respectively, $E_{\mathrm{F}}$ is the Fermi level, and $\Phi_{\mathrm{B}}$ is the Schottky barrier height.

In this article, we present the fabrication and characterisation of IPE-based Schottky detectors where the contact electrode is formed as electrically-contacted metal nanomonopoles. This device uses hot holes arising from plasmon decay to directly generate the photocurrent, resulting in the detection of subbandgap infrared light. To reduce the dark current, we fabricated the probing arm of the device as a bilayer Au/Ti structure having a higher Schottky barrier than the Au nanoantenna array.

Design. Fig. 2 shows a schematic of the proposed structure. It includes an array of nanomonopoles for guiding SPPs on a lightlydoped p-Si epitaxial layer on a heavily-doped p+-Si substrate. The $\mathrm{Au}$ nanomonopoles are interconnected via $\mathrm{Au}$ lines running perpendicularly to their axes through the middle of each to a common circular contact pad used to collect the photocurrent. A nanomonopole is designed to couple normally-incident $p$ polarised infrared ( $\lambda_{0} \sim 1550 \mathrm{~nm}$, sub-bandgap) light to SPPs propagating along the bottom surface such that the SPP fields are coincident with the Schottky contact. As SPPs propagate they are absorbed in the metal, generating hot carriers therein primarily along the Schottky contact. Detection then occurs through the collection of hot holes in p-Si via IPE. The substrate is placed on a backside $\mathrm{Al} \mathrm{Ohmic}$ ground contact.

The proposed photodetector was analyzed by investigating its electromagnetic performance over a range of wavelengths. The right inset to Fig. 2 shows the absorptance spectra for a good design of length $125 \mathrm{~nm}$, width $44 \mathrm{~nm}$ and thickness of $20 \mathrm{~nm}$. The array consisted of 1600 nanomonopoles arranged in a $40 \times 40$ array with a $300 \mathrm{~nm}$ inter-antenna spacing in both the longitudinal and transverse directions, sufficient to ensure that near-field interantenna coupling is absent.

The dark current of a Schottky diode is given by:

$$
I_{D}=A_{c} A^{* *} T^{2} e^{-\frac{q \phi_{B}}{n k T}}
$$

where $k$ is Boltzmann's constant, $n$ is the ideality factor of diode, $T$ is the absolute temperature, $A^{* *}$ is the effective Richardson constant ( $32 \mathrm{Acm}^{-2} \mathrm{~K}^{-2}$ for holes [14]) and $A_{c}$ is the Schottky contact area. The probing structure (pad and arm) is made of $\mathrm{Au} / \mathrm{Ti}$ in order to reduce its dark current (Ti produces a significantly higher Schottky barrier height on $\mathrm{p}-\mathrm{Si}$ than $\mathrm{Au}$ ). The dark current is then determined by the sum of two terms: one due to the $\mathrm{Au} / \mathrm{Ti}$ probing structure and the other by the $\mathrm{Au}$ nanomonopole array. The contact areas are $45.5 \mu \mathrm{m}^{2}$ for the $\mathrm{Au} / \mathrm{Ti} / \mathrm{p}$-Si structure and 4.5 $\mu \mathrm{m}^{2}$ for the $\mathrm{Au} / \mathrm{p}-\mathrm{Si}$ electrically-contacted nanomonopole array. Consequently, a dark current of $I_{\mathrm{d}}=0.75 \mathrm{nA}$ and $3.8 \mu \mathrm{A}$ are obtained at $300 \mathrm{~K}$, respectively [14]. The low barrier height of the nanomonopole array should permit a detection window covering the entire shortwave infrared spectral range ( $\lambda=1.2$ to $2.5 \mathrm{~mm})$.

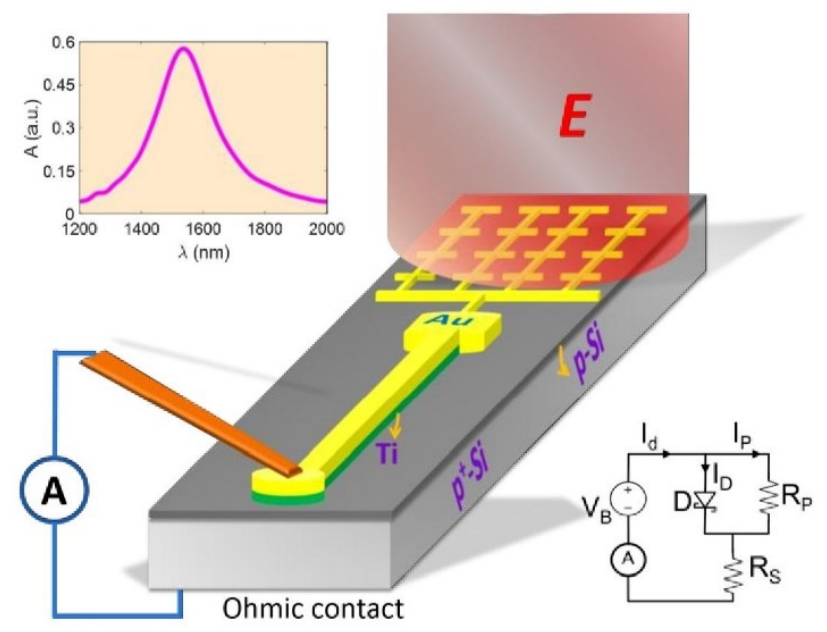

Fig. 2. Schematic of an Au-Ti/p-Si optical nanoantenna Schottkycontact diode for sub-bandgap detection. The materials used are Au for the nanoantenna array, $\mathrm{p}-\mathrm{Si}$ for the epitaxial layer, $\mathrm{p}^{+}-\mathrm{Si}$ for the substrate and $\mathrm{Au} / \mathrm{Ti}$ for the probing structure (pad and arm). The device is illuminated from the top with polarised infrared light aligned to the nanomonopole lengths. A good design of length $125 \mathrm{~nm}$, width $44 \mathrm{~nm}$ and thickness $20 \mathrm{~nm}$ produces a resonant wavelength at $\lambda=$ $1534 \mathrm{~nm}$ as identified from the absorptance response in inset.

Fabrication. The devices were fabricated on a double-side polished wafer, consisting of a p $\mathrm{p}^{+}-\mathrm{Si}$ substrate $250 \pm 25 \mu \mathrm{m}$ thick, of acceptor density $N_{\mathrm{A}}=5 \times 10^{18}$ to $3.5 \times 10^{18} \mathrm{~cm}^{-3}$, bearing a p-Si epitaxial layer $3 \pm 0.5 \mu \mathrm{m}$ thick, of acceptor density $N_{\mathrm{A}}=1.7 \times 10^{15}$ to $1.0 \times 10^{15} \mathrm{~cm}^{-3}$. Sintered Al was used for the Ohmic contact on the backside. The $\mathrm{Au} / \mathrm{Ti}$ probing structures were fabricated on the $\mathrm{p}-\mathrm{Si}$ epitaxial surface using a bilayer liftoff lithography technique and a UV contact mask aligner to expose the resist. Au/Ti was deposited by e-beam evaporating $5 \mathrm{~nm}$ of $\mathrm{Ti}$ followed by thermally evaporating $15 \mathrm{~nm}$ of $\mathrm{Au}$. The layout of the electrically-contact $\mathrm{Au}$ nanomonopoles was prepared using e-LiNE software [15] and ebeam lithography was used to define them in a bilayer PMMA liftoff process. The layout of this portion overlapped with the probing structure to ensure electrical continuity. For nanomonopoles array, immediately prior to Au deposition, the sample was placed in a buffered hydrofluoric (HF) acid solution to remove any native $\mathrm{SiO}_{2}$ and allow intimate contact between the $\mathrm{Au}$ and the semiconductor. No cover layer was used to allow for direct electrical probing and photocurrent measurements. 
Fig. 3 shows scanning electron microscope (SEM) images of a fabricated structure. The metal thickness and roughness were deduced from atomic force microscope (AFM) scans of the nanoantenna array (e.g., Fig. 3), revealing a thickness of $\sim 17 \mathrm{~nm}$ with a root mean square (RMS) roughness of $1 \mathrm{~nm}$ for each monopole (design target $=20 \mathrm{~nm}$ ). All other dimensions are well in keeping with the target ones.

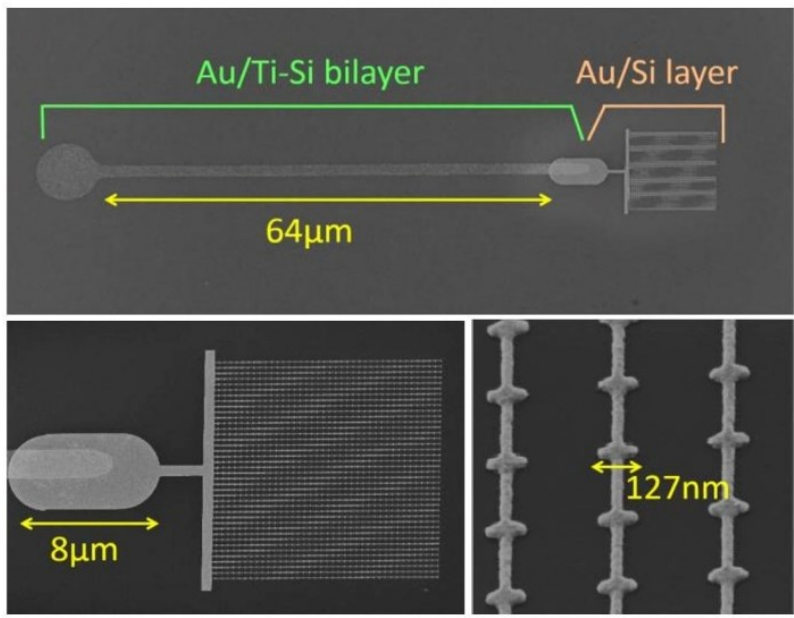

Fig. 3. SEM images of a representative nanoantenna array on p-Si: (top) overall view of the device consisting of a bilayer $\mathrm{Au} / \mathrm{Ti}$ probing structure, and (bottom) an array of $\mathrm{Au}$ electrically-contacted nanomonopole antennas.

Measurement A nanomonopole array under test is first biased by probing its probe pad and grounding its Al contact using a source meter (Fig. 2). A cleaved polarisation-maintaining single-mode fibre (PM-SMF) is then used to illuminate the nanoantenna under test from the top. A six-axis micro-positioner is manipulating the fiber. A tunable external cavity laser is used to generate the incident light at $\lambda_{0} \sim 1550 \mathrm{~nm}$. The laser is thermally stabilized and linearly polarized, with the polarisation aligned along the slow axis of the PM-SMF. The setup is placed on a vibration isolation optical table and is shielded from background light. Instrument control and data acquisition are performed via computer using LabVIEW (SP1 2013) [16]. A power meter was used to determine the optical power incident on a photodetector after removing the losses of all the elements in the input path of the setup.

The experiments are conducted by placing an array under test under microscope and using tipless AFM cantilever probes attached to a micropositioner to make electrical contact to a device on its probe pad. A reverse-bias voltage is applied between the probe pad and the Al Ohmic contact on the bottom surface (ground). Another micropositioner is then used to align the PMSMF (polarisation-aligned to launch TM light) to the photodetector. The cleaved fibre output field behaves as a Gaussian beam, with the beam waist coincident with the fibre output facet. The fibre is aligned to the nanomonopole array by placing the device under reverse-bias and maximising the measured photocurrent. It is expected that when the fibre is rotated to produce TE-polarised incidence, no photocurrent is generated. Once the best possible alignment is achieved, a computer automated routine controlling ancillary instrumentation is run to rapidly execute test routines and gather measurement data. Ancillary instruments consisted of a laser tunable over $\sim 100 \mathrm{~nm}$ (Photonetics TUNIC-Plus) and a source meter (Keithley 2400) used to apply a bias voltage and read the total current (dark + photo) simultaneously. All measurements were obtained at room temperature.

The black curve in Fig. 4 shows an average of five measured $I-V$ curves for a nanoantenna photodetector without illumination. It shows the rectifying property of the Schottky structure. The reverse bias characteristics are soft for all tested devices, due to the low Schottky barrier and possibly to deep traps formed by the diffusion of Au into p-Si during fabrication [17]. The total contact area of our test device is $A_{c}=49.5 \mu \mathrm{m}^{2}$. To obtain a lower dark current, we designed and fabricated the probing structure as a bilayer of $\mathrm{Au} / \mathrm{Ti}$, which forms a higher Schottky barrier on $\mathrm{p}-\mathrm{Si}$ than $\mathrm{Au}$. The area of the probing structure is $\sim 45.5 \mu \mathrm{m}^{2}$ which corresponds to more than $90 \%$ of the entire device area. Taking $\Phi_{\mathrm{B}}$ $=0.62 \mathrm{eV}$ for Ti $/ \mathrm{p}-\mathrm{Si}$ and $\Phi_{\mathrm{B}}=0.32 \mathrm{eV}$ for Au$/ \mathrm{p}-\mathrm{Si}$ [14] produces $I_{\mathrm{d}}$ $=0.75 \mathrm{nA}$ for the Ti $/ \mathrm{p}$-Si probing structure and $I_{\mathrm{d}}=3.8 \mu \mathrm{A}$ for the $\mathrm{Au} / \mathrm{p}-\mathrm{Si}$ electrically-contacted nanoantenna array. By the way, using bilayer structure for probe purpose makes the dark current of whole device $5 \times$ lower than for the corresponding Au-only design, resulting in an improved signal-to-noise ratio (SNR) for the photodetector.

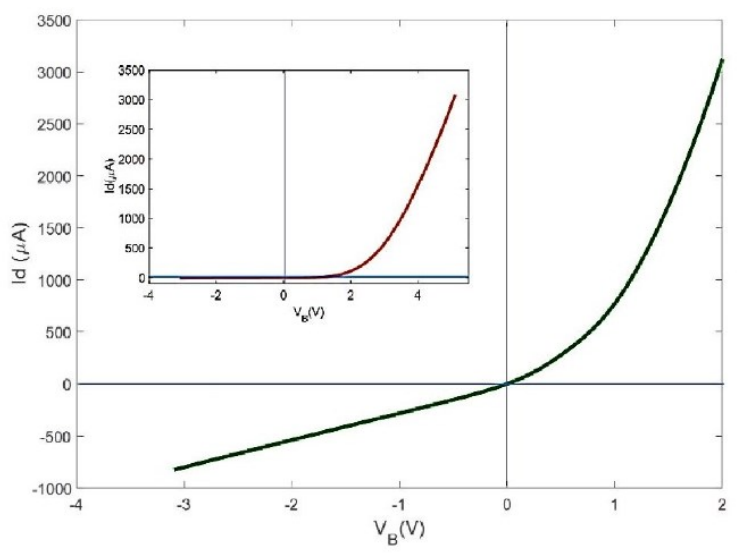

Fig. 4. The black curve shows an average of five dark $I-V$ characteristics for a complete device. The inset shows the dark $I-V$ characteristics of the probing structure only.

The reverse bias behaviour of the Schottky nanoantenna under test does not show a strong rectifying property. Because the experimental setup and the photodetectors have a high resistance, solving Eq. (1) to obtain $\Phi_{\mathrm{B}}$ is not very accurate. The reverse bias and small forward bias regimes are affected by the shunt current $I_{P}$, and the shunt and series resistances, $R_{P}$ and $R_{S}$, of the equivalent circuit of a non-ideal diode shown in left inset of Fig. 2 [17]. Several methods can be applied to extract the barrier height of high resistance Schottky diodes. The method proposed by Werner [18] has been shown to yield accurate results. Following this method yields $R_{P}=3.5 \mathrm{k} \Omega, R_{S}=311 \Omega$ and $\Phi_{\mathrm{B}}(\mathrm{Si})=0.32 \mathrm{eV}, \Phi_{\mathrm{B}}(\mathrm{Ti})=0.57 \mathrm{eV}$. The ideality factor was found to be 3.18 and 0.91 for $\mathrm{Au} / \mathrm{Si}$ and $\mathrm{Ti} / \mathrm{Si}$, respectively. Given that $h v>\Phi_{\mathrm{B}}$ must be satisfied in order for the SPPs to be detected, the long-wavelength cut-off of the photodetectors is $\lambda_{0} \sim 3880 \mathrm{~nm}$ implying a broad range of operating wavelengths limited only by the nanoantenna design.

Fig. 5 plots the $p$-polarized photocurrent response measured at normal incidence with $P_{\mathrm{inc}}=1.6 \mathrm{~mW}$ and $V_{\mathrm{B}}=-10 \mathrm{mV}$ for two 
detectors having arrays of nanomonopoles of different dimensions: each monopole in both arrays are $\sim 45 \mathrm{~nm}$ wide and $17 \mathrm{~nm}$ thick, the shorter ones are $\sim 124 \mathrm{~nm}$ long and the longer ones are $130 \mathrm{~nm}$. Two wavelength dependencies are observed on each response. The rapidly varying dependencies come from Fabry-Perot resonances. In fact, light transmitted through the array propagates through the semi-transparent substrate $\left(\mathrm{p}^{+}-\mathrm{Si}\right)$ and is reflected from the backside Ohmic contact. The free spectral range (FSR) of the Fabry-Perot responses (given by the difference between the frequencies of two adjacent peaks) is $\sim 2 \mathrm{THz}$. The Fabry-Perot resonances would disappear if a more heavily doped Si substrate was used. The second wavelength dependency is the slow one produced by the monopole resonance over their length. Increasing the monopole length, red-shifts the resonance wavelength, as expected.
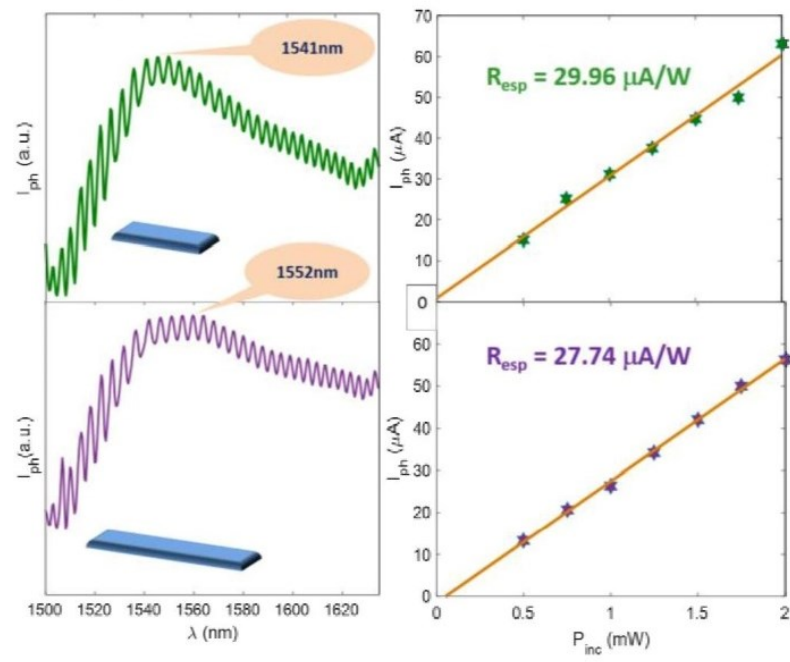

Fig. 5. Measured photocurrent response (left panels) of two electrically-contacted nanomonopole array photodetectors $\left(V_{\mathrm{B}}=-10\right.$ $\mathrm{mV}$ ). The nanomonopoles are $45 \mathrm{~nm}$ wide, $17 \mathrm{~nm}$ thick and $124 \mathrm{~nm}$ long (top panels) or $130 \mathrm{~nm}$ long (bottom panels). The rapid wavelength variations correspond to Fabry-Perot resonances. The corresponding photocurrent vs. incident power are also plotted (right panels); the slope of the linear fit gives the responsivity of each device.

Typical responsivity measurement results are also shown in Fig. 5. The slope of the curves yields the responsivity. We fit the measured data points to a linear model and take the slope of the fit as the responsivity of the photodetectors, yielding $R_{\text {esp }}=29.96$ and $27.74 \mu \mathrm{W} / \mathrm{A}$ for the two photodetectors.

In summary, we have presented a broadband $\mathrm{Si}$ plasmonic Schottky photodetector operating in the sub-bandgap regime and exploiting IPE. A nanomonopole antenna array is used to couple perpendicularly-incident light to SPPs propagating along an array of $\mathrm{Au}$ nanomonopoles forming a Schottky contact to $\mathrm{p}-\mathrm{Si}$. The responsivity is enhanced by the absorption of SPPs directly along the Schottky contact leading to the creation of hot holes near the contact. These holes cross over the Schottky barrier $(\sim 0.32 \mathrm{eV})$ and are collected as a photocurrent. Our measurements produced a responsivity of $\sim 30 \mu \mathrm{A} / \mathrm{W}$ at telecom wavelengths $(\sim 1550 \mathrm{~nm})$ and under low reverse bias. The optical bandwidth (FWHM) is $\sim 45 \mathrm{~nm}$. The dark current performance of the photodetector has been optimized by using a smaller area and fabricating the probing structure with Ti which produces a higher Schottky barrier to $\mathrm{p}-\mathrm{Si}$. The range of potential applications of this device concept is diverse. As a Si-based photodetector capable of detecting subbandgap photons, this structure is a good candidate for on-chip silicon photonics, ultimately eliminating the need to integrate additional semiconductor materials as detectors into chip designs, which would lower fabrication costs. The broad infrared sensitivity of these devices could enable low-cost silicon infrared imaging biosensors. The IPE mechanism employed is compatible with existing, above-bandgap photodetectors, which when combined could greatly extend the spectral range of silicon lightharvesting devices, such as Si-based solar cells, into the infrared region of the spectrum.

Acknowledgment. The authors are grateful to Ewa Lisicka-Skrzek for assistance with the photolithography of samples.

\section{References}

1. L. Novotny and N. van Hulst, Nat. Photonics, 5, (2011).

2. M. Alavirad, L. Roy and P. Berini, J. Photon. Energy. 6, 1 (2016).

3. D. Arble, N. Berkovitch, A. Nevet, A. Peer, S. Cohen, D. Ritter and M. Orenstein, Opt. Express 19, 10 (2011).

4. A. Kinkhabwala, Z. Yu, S. Fan, Y. Avlasevich, K. Mullen and W.E. Moerner, Nat. Photonics 3, (2009).

5. M.W. Knight, H. Sobhani, P. Nardlander and N.J. Halas, Science 332, (2011).

6. P. Bharadwaj, B. Deutsch and L. Novotny, L., Adv. Opt. Phot. 1, (2009).

7. A. Ahmed and R. Gordon, Nano Lett., 11, 4 (2011).

8. A. Andryieuski, R. Malureanu, G. Biagi, T. Holmgaard and A. Lavrinenko, Opt. Lett. 37, 6 (2012).

9. M. Alavirad; S. S. Mousavi; L. Roy and P. Berini, Opt. Express 21, 4, (2013).

10. K. T. Lin, H. L. Chen, Y. S. Lai and C. C. Yu, Nat. Commun. 5, (2013).

11. S. S. Mousavi, A. Stöhr and P. Berini, Appl. Phys. Lett. 104, 14 (2014).

12. S. S. Mousavi, P. Berini and D. McNamara, Opt. Express 20, 16 (2012).

13. P. Berini, Phys. Rev. B 63, 125417 (2001).

14. S. M. Sze and K. K. Ng, Physics of Semiconductor Devices. (2006)

15. e_LiNE; Raith nanofabrication: (Accessed 2015)

16. LabVIEW; National Instruments Corporation (Accessed 2016)

17. M. Alavirad, A. Olivieri, L. Roy and P. Berini, Opt. Express., 24, 20 (2016).

18. J. H. Werner, Appl. Phys. A. 47, 3 (1988). 


\section{Chapter: Conclusions and Future Work}

\subsection{Concluding remarks}

The preceding articles presented the development of two types of photodetectors: grating coupler based detectors and nanoantenna based detectors, which, both, in terms of responsivity and operating wavelength, are the first device of these types to be demonstrated in the literature:

- A novel plasmonic Schottky photodetector consisting of a Au nanograting on a thin metal patch on $p$-type Si was proposed. This device provides detection via the IPE of hot holes generated in the patch along the Schottky contact. This structure produced some of the highest responsivities reported to date in the literature, of about $13 \mathrm{~mA} / \mathrm{W}$ at $\lambda_{0} \sim 1550 \mathrm{~nm}$ (with low electrical bias). Gathered into arrays, these small area devices are capable of aggregate bandwidths into the $\mathrm{Tb} / \mathrm{s}$ range. These detectors have many important advantages such as simplicity, compatibility with silicon, and low-cost fabrication.

- A highly sensitive design using the concept of an optical nanoantenna was proposed. Arrays of Au nanodipole antennas form a Schottky contact to Si. They have been investigated theoretically and experimentally for use as a wavelength-selective subbandgap photodetector and biosensor. Photodetection occurs via IPE for photon energies above the Schottky barrier height but below the bandgap of Si. As a biosensor, the nanoantenna structure offers significant advantages as it simplifies the interrogation set-up: only the photocurrent generated by the device need be monitored, and the excitation optics cane be located at the bottom and are physically separated from the micro-fluidics which can be located at the top of the device. Compelling sensitivities are predicted for dipoles: $250 \mathrm{~nm} / \mathrm{RIU}$ of bulk sensitivity and $8 \mathrm{~nm} / \mathrm{nm}$ of surface 
sensitivity (biochemical adlayer in $\mathrm{H}_{2} \mathrm{O}$ ). Also, using a transmission line model to represent the dipole antennas, we derive analytical expressions for the bulk and surface sensitivities of the structure and validate them via comparisons with numerical results. As a detector, we showed that this small structure is capable of producing a highresponsivity device on silicon at telecommunication wavelengths.

The main contributions of this thesis could be listed as follows:

- A comprehensive surveying and comparing of most recent cutting-edge surface plasmon-polaritons IPE-based photodetectors is reported;

- Design, fabrication, measurement of a fast and high-responsivity sub-bandgap plasmonic photodetectors operating at telecommunication wavelengths are presented;

- Design, fabrication, measurement of a high-sensitivity Schottky-contact IPE-based plasmonic nanoantenna, targeting telecom and biosensing applications is reported;

- Optimization theory of a high-sensitivity nanoantenna based Schottky-contact photodetectors targeting commercial substrate and wavelengths is reported;

\subsection{Future work}

In view of the main contributions of the present thesis, the author makes the following recommendations for future work:

- Additional experimentation can be done to optimize the grating coupler detectors. Perpendicular excitation is an efficient illumination, but the small size of these 
detectors requires more precise optical positioners and polarization control (and consequently absorbed power).

- The top surface of the grating ridges is rough, which makes the spectral responses broader than expected. It is a strong function of the metal evaporation step and can be improved by using a better fabrication process.

- A more complex experimental setup is needed to evaluate the device's time response for potential use in a short-range high-speed communication link.

- In addition to investigating nanoantenna devices as high-responsivity photodetectors, a full theoretical investigation of their use as a biosensor was also carried out in this thesis. A natural next step would be to fabricate and test such biosensors.

- Electrically contacting the photodetectors was difficult as the probe tip touches, scratches, hides or even destroys the device. Also, adding a probe and positioner to the experimental setup introduces drift and difficulties in establishing a stable measurement. The author proposes a different probing technique to minimize these effects to investigate the dark current and photocurrent (ac and dc) in an accurate manner.

- Throughout the thesis, we assumed a room temperature for calculations and measurements but it would be very important and worthwhile to consider this factor for both photodetectors. Noise - thermal or shot - can be affected by temperature and, specifically in the electrical characterization, can be a significant problem. Dark current level goes high and monitoring the power peak would be difficult. We predict some 
challenging issues related to temperature effects. For example, study of electrical bandwidth and sensitivity of these photodetectors versus temperature would be needed. 


\section{References}

[1] Casalino, M., Coppola, G., Iodice, M., Rendina, I., and Sirleto, L., "Critically coupled silicon fabryperot photodetectors based on the internal photoemission effect at $1550 \mathrm{~nm}$," Opt. Exp., vol. 20, pp.12599-609, 2012

[2] A. Akbari, P. Berini, "Schottky contact surface-plasmon detector integrated with an asymmetric metal stripe waveguide," Appl. Phys. Lett. 95, 021104, 2009

[3] C. Daboo, M. Baird, H.H.M. Apsley, M. Emeny, "Improved surface plasmon enhanced photodetection at an AuGaAs Schottky junction using a novel molecular beam epitaxy grown onto coupling structure," Thin Solid Films 201, 9 - 27, 1991

[4] S.R.J Brueck, V. Diadiuk, T. Jones, W Lenth, "Enhanced quantum efficiency internal photoemission detectors by grating coupling to surface plasma waves," Appl. Phys. Lett. 46, 915-917, 1985

[5] M. Tsuneyasu, C. Sasakawa, N. Naruishi, Y. Tanaka, Y. Yoshida and Keiko Taw, "Sensitive detection of interleukin- 6 on a plasmonic chip by grating-coupled surface-plasmon-field-enhanced fluorescence imaging", Jpn. J. Appl. Phys. 53, 2014

[6] A. Akbari, R.N. Tait, P. Berini, "Surface plasmon waveguide Schottky detector," Opt. Exp. 18, $8505-14,2010$.

[7] I. Goykhman, B. Desiatov, J. Khurgin, J. Shappir, U. Levy, "Waveguide based compact silicon Schottky photodetector with enhanced responsivity in the telecom spectral band," Opt. Exp., Vol. 20, Issue 27, pp. 28594-28602, 2012

[8] M. W. Knight, H. Sobhani, P. Nordlander, N.J. Halas, "Photodetection with active optical antennas," Science 332, 702-704, 2011

[9] S. Lal, S. Link, and N. J. Halas, "Nano-optics from sensing to waveguiding," Nat Photon 1, 641648, 2007. 
[10] C.-Y. Tsai, S.-P. Lu, J.-W. Lin, and P.-T. Lee, "High sensitivity plasmonic index sensor using slablike gold nanoring arrays," Appl. Phys. Lett. 98, 153108, 2011

[11] B. Desiatov, I. Goykhman, N. Mazurski, J. O. Shappir, J.B. Khurgan, U. Levy, "Plasmonic enhanced silicon pyramids for internal photoemission Schottky detectors in the near-infrared regime", Optica Lett. Vol. 2, No. 4, 2015

[12] M.A. Nazirzadeh, F.B. Atar, B.B. Turgut and A.K. Okyay, "Random sized plasmonic nanoantennas on Silicon for low-cost broad-band near-infrared photodetection”, Scient. Rep. 4, Article number: 7103, (2013).

[13] J. Derov, Y. Y. Teng, and A. S. Karakashian, "Angular scan spectrum of a surface plasma excitation on a Schottky diode", Phys. Lett. A 95, 197-200, 1983

[14] P. Dawson, G. F. Cairns, and S. M. O'Prey, "Prism coupler with variable coupling gap", Rev. Sci. Instr. 71, 4208-4212, 2000

[15] A. Sellai and P. Dawson, "Quantum efficiency in GaAs Schottky photodetectors with enhancement due to surface plasmon excitations", Solid-State Electr. 46, 29-33, 2002

[16] P. Berini, "Surface plasmon photodetectors and their applications", Laser \& Photon. Rev. 8 (2), $197-220,2014$

[17] C. Scales, I. Breukelaar, and P. Berini, "Surface-plasmon Schottky contact detector based on a symmetric metal stripe in silicon," Opt. Lett., vol. 35, pp. 529-531, (2010)

[18] A. Akbari and P. Berini, "Schottky contact surface-plasmon detector integrated with an asymmetric metal stripe waveguide," Appl. Phys. Lett., vol. 95, 021104, 2009

[19] A. Akbari, A. Olivieri, Pierre Berini, "Subbandgap Asymmetric Surface Plasmon Waveguide Schottky Detectors on Silicon,” IEEE J. Sel. Top. Quant. Electron, Vol. 19, No. 3, 2013

[20] S. A. Maier, "Plasmonics: Fundamentals and Applications", Springer Science+Business Media, New York, 2007

[21] J. W. Yoon, W. J. Park, K. J. Lee, S. H. Song, and R. Magnusson, “Surface-plasmon mediated total absorption of light into silicon," Opt. Express 19, 20673-20680, 2011 
[22] X. G. Guo, R. Zhang, J. C. Cao, et. al. "Surface plasmon-enhanced absorption in metal grating coupled terahertz quantum well photodetectors", IEEE J. Quant. Electron., 48, 1113, 2012

[23] A. Takeda, T. Aihara, M. FukuharaY. Ishii, M. Fukuda, "Sensitivity improvement of Schottkytype plasmonic detector," Proc. SPIE 8923, Micro/Nano Materials, Devices, and Systems, 89234G, 2013.

[24] A. Takeda, T. Aihara, M. FukuharaY. Ishii, M. Fukuda, "Schottky-type surface plasmon detector with nano-slit grating using enhanced resonant optical transmission," J. Appl. Phys. 116, 084313, (2014).

[25] M. Fukuhara, M. Ota, H. Sakai, T. Aihara, Y. Ishii, M. Fukuda, "Low-loss waveguiding and detecting structure for surface plasmon polaritons," Appl. Phys. Lett. 104, 081111, (2014)

[26] J. Hetterich, G. Bastian, N. A. Gippius, S. G. Tikhodeev, G. von Plessen, and U. Lemmer, “Optimized design of plasmonic MSM photodetector," IEEE J. Quant. Electron. 43, 855-859, 2007

[27] K. Berthold, W. Beinstingl, and E. Gornik, "Frequency- and polarization-selective Schottky detectors in the visible and near ultraviolet," Optics Lett. Vol. 12, Issue 2, pp. 69-71, 1987

[28] D. Okamoto, J. Fujikata, K. Nishi, and K. Ohashi, "Numerical study of near-infrared photodetectors with surface-plasmon antenna for optical communication," Jpn. J. Appl. Phys., vol. 47, no. 4, pp. 2921-2923, Apr. 2008.

[29] A. M. Glass, P. F. Liao, D. H. Olson, L. M. Humphrey, "Optical metal-oxide tunnel detectors with microstructured electrodes," Opt. Lett. 7, 575-577, 1982

[30] H. Satoh and H. Inokawa, "Surface Plasmon Antenna with Gold Line and Space Grating for Enhanced Visible Light Detection by a Silicon-on-Insulator Metal-Oxide-Semiconductor Photodiode," IEEE Trans. Nanotechnol. 11, 346-351, 2012

[31] P. Genevet, J. Lin, M. A. Kats, and F. Capasso, "Holographic detection of the orbital angular momentum of light with plasmonic photodiodes," Nature Comm. 3, 1278, 2012

[32] M. Pelton, J. Aizpurua, G. Bryant, "Metal-nanoparticle plasmonics," Las. \& Photon. Rev., Vol 2, Issue 3, 136-159, 2008 
33. S. A. Maier, Plasmonics: Fundamentals and Applications, Springer Science+Business Media, New York (2007).

34. D. K. Gramotnev and S. I. Bozhevolnyi, "Nanofocusing of electromagnetic radiation," Nat. Photon., 4, 83 (2010).

35. A. Akbari, R.N. Tait and P. Berini, "Surface plasmon waveguide Schottky detector," Opt. Exp. 18, 8505-14, (2010).

36. A. Olivieri, C. Chen, S. Hassan, E. Lisicka-Skrzek, R. N. Tait, and P. Berini, "Plasmonic Nanostructured Metal-Oxide-Semiconductor Reflection Modulators,” Nano Lett., 15 (4), pp 2304$2311(2015)$

37. P. Berini, A. Olivieri, and C. Chen, "Thin Au surface plasmon waveguide Schottky detectors on $p$ Si," Nanotechnology 23, 444011 (2012).

38. H. A. Atwater and A. Polman, "Plasmonics for improved photovoltaic devices," Nature Mater. 9, 205 (2010).

39. S. W. Baek, J. Noh, C.H. Lee, B. S. Kim, M.K. Seo, and J.Y. Lee, "Plasmonic Forward Scattering Effect in Organic Solar Cells: A Powerful Optical Engineering Method," Sci. Rep. 3, 1726 (2013).

40. A. J. Leenheer, P. Narang, N. S. Lewis and H. Atwater, "Solar energy conversion via hot electron internal photoemission in metallic nanostructures: Efficiency estimates," J. Appl. Phys 115, 134301 (2014).

41. M. Alavirad; S. S. Mousavi; L. Roy and P. Berini, "Schottky-contact plasmonic dipole rectenna concept for biosensing," Opt. Express 21 (4), 4328-4347 (2013).

42. A. E. Cetin, A. F. Coskun, B. C. Galarreta, M. Huang, D. Herman, A. Ozcan, and H. Altug, "Handheld high-throughput plasmonic biosensor using computational on-chip imaging," Light Sci. Appl. 3, e122 (2014).

43. M. Alavirad, L. Roy, P. Berini, "Optimization of Plasmonic Nanodipole Antenna Arrays for Sensing Applications," IEEE J. Sel. Top. Quant., 20 (3), 7-14, (2014). 
44. WR Wong, SD Sekaran, FRM Adikan and P Berini, "Detection of dengue NS1 antigen using longrange surface plasmon waveguides," Biosensors and Bioelectronics 78, 132-139, (2016).

45. WR Wong, FRM Adikan and P Berini, "Long-range surface plasmon Y-junctions for referenced biosensing," Optics express 23 (24), 31098-31108, (2015).

46. P. Béland, O. Krupin and P. Berini, "Selective detection of bacteria in urine with a long-range surface plasmon waveguide biosensor," Biomedical Optics Express 6 (8), 2908-2922, (2015).

47. P. Berini, Adv. Opt. Phot. 1, 484-588 (2009).

48. T. Aihara, M. Fukuhara, A. Takeda, B. Lim, M. Futagawa, Y. Ishii, K. Sawada, and M. Fukuda, "Monolithic Integration of Surface Plasmon Detector and Metal-Oxide-Semiconductor Field-Effect Transistors," IEEE Photon. J. 5, 6800609 (2013).

49. M. Toma, K. Toma, P. Adam, J. Homola, W. Knoll, and J. Dostalek, "Surface plasmon-coupled emission on plasmonic Bragg gratings.," Opt. Express 20, 14042 (2012).

50. Z. Liu, D. Xi, D. Pile, Q. Luo, N. Fang, and X. Zhang, "Enhanced backward scattering by surface plasmon on silver film," Appl. Phys. A 87, 157 (2007).

51. H. Hu, X. Zeng, D. Ji, L. Zhu, and Q. Gan, "Efficient end-fire coupling of surface plasmons on flat metal surfaces for improved plasmonic Mach-Zehnder interferometer,” J. Appl. Phys. 113, 053101,( 2013).

52. T. Aihara and M. Fukuda, "Transmission properties of surface-plasmon-polariton coherence," Appl. Phys. Lett. 100, 213115, (2012).

53. K. L. Kelly, E. Coronado, L. L. Zhao, and G. C. Schatz, "The Optical Properties of Metal Nanoparticles: The Influence of Size, Shape, and Dielectric Environment," J. Phys. Chem. B 107, 668677, (2003).

54. M. Pelton, J. Aizpurua, and G. Bryant, "Metal-nanoparticle plasmonics," Laser \& Phot. Rev. 2, 136-159, (2008).

55. S. S. Mousavi, A. Stöhr and P. Berini, "Plasmonic photodetector with terahertz electrical bandwidth", Applied Physics Letters 104 (14), 143112, (2014) 
56. P. Bharadwaj, B. Deutsch, and L. Novotny, "Optical Antennas," Adv. Opt. Phot. 1, 438-483, (2009).

57. C. Clavero, "Plasmon-induced hot-electron generation at nanoparticle/metaloxide interfaces for photovoltaic and photocatalytic devices," Nat. Photon. 8, 95-103 (2014).

58. A. Sobhani, M. Knight, Y. Wang, B. Zheng, N. S. King, L. V. Brown, Z. Fang, P. Nordlander and N. J. Halas, "Narrowband photodetection in the near-infrared with a plasmon-induced hot electron device," Nat. Commun. 4, 1643 (2013).

59. M. Casalino, G. Coppola, M. Iodice, I. Rendina, and L. Sirleto, "Near-infrared sub-bandgap allsilicon photodetectors: state of the art and perspectives," Sensors 10, 10571 (2010).

60. M. W. Knight, H. Sobhani, P. Nordlander and N. J. Halas, "Photodetection with active optical antennas," Science 332, 702-704, (2011).

61. A. M. Brown, R. Sundararaman, P. Narang, W. A. Goddard, and H. A. Atwater, "Nonradiative Plasmon Decay and Hot Carrier Dynamics: Effects of Phonons, Surfaces, and Geometry," ACS Nano, 10 (1), pp 957-966, (2016).

62. M. L. Brongersma, N. J. Halas, P. Nordlander, "Plasmon-induced hot carrier science and technology," Nat. Nanotechnol. 10, 25-34 (2015)

63. P. Berini, "Surface plasmon photodetectors and their applications," Laser \& Photon. Rev., 8(2), $197-220(2014)$

64. P. Berini, "Figures of merit for surface plasmon waveguides," Opt. Express 14, 13030-13042 (2006)

65. T. Gong and J. N. Munday, "Materials for hot carrier plasmonics," Opt. Mater. Express, Vol. 5, Issue 11, pp. 2501-2512, (2015).

66. R. Sundararaman, P. Narang, A. S. Jermyn, W. A. Goddard, and H. A. Atwater, "Theoretical predictions for hot-carrier generation from surface plasmon decay," Nat. Commun. 5(5788), (2014).

67. S. M. Sze, [Physics of Semiconductor Devices], Wiley, New York (1981).

68. C. Scales and P. Berini, "Thin-film Schottky barrier photodetector models," IEEE J. Quant. Electr. 46, 633-643 (2010). 
69. B. Y. Zheng, H. Zhao, A. Manjavacas, M. McClain, P. Nordlander, and N. J. Halas, "Distinguishing between plasmon-induced and photoexcited carriers in a device geometry," Nat. Commun. 6(7797), (2015).

70. J. E. Sipe and J. Becher, "Surface-plasmon-assisted photoemission," J. Opt. Soc. Am. 71, 12861288 (1981).

71. S. Oldenburg, R. Averitt, S. L. Westcott and N. J. Halas, "Nanoengineering of optical resonances," Chem. Phys. Lett. 288, 243-247 (1998).

72. M. Moskovits, Surface enhanced spectroscopy. Rev. Mod. Phys. 57, 783-826 (1985).

73. J. I. Gersten and A. Nitzan, "Photophysics and photochemistry near surfaces and small particles," Surf. Sci. 158, 165-189 (1985).

74. M. Bernardi, J. Mustafa, J. B. Neaton and S. G. Louie, "Theory and computation of hot carriers generated by surface plasmon polaritons in noble metals," Nat. Commun. 6(7044), (2015)

75. X. X. D. Li, Z. Zhang, "Landau damping of quantum plasmons in metal nanostructures," New J. Phys. 15, 023011 (2013).

76. A. Manjavacas, J. G. Liu, V. Kulkarni, and P. Nordlander, "Plasmon-Induced Hot Carriers in Metallic Nanoparticles,” ACS Nano, 8 (8), pp 7630-7638, (2014).

77. C. F. Bohren, "How can a particle absorb more than the light incident on it?" Am. J. Phys. 51, 323327 (1983).

78. P. Dombi, A. Hörl, P. Ra' cz, I. Ma'rton, A. Trügler, J. R. Krenn and U. Hohenester, "Ultrafast strong-field photoemission from plasmonic nanoparticles," Nano Lett. 13, 674-678 (2013).

79. H. Zhang, A. O. Govorov, "Optical generation of hot plasmonic carriers in metal nanocrystals: The effects of shape and field enhancement," J. Phys. Chem. C 118, 7606-7614 (2014).

80. M. Casalino, G. Coppola, M. Iodic, I. Rendina and L. Sirleto, "Critically coupled silicon fabryperot photodetectors based on the internal photoemission effect at $1550 \mathrm{~nm}$," Opt. Exp., vol. 20, pp.12599-609 (2012) 
81. C. Daboo, M. Baird, H.H.M. Apsley, M. Emeny, "Improved surface plasmon enhanced photodetection at an AuGaAs Schottky junction using a novel molecular beam epitaxy grown onto coupling structure," Thin Solid Films 201, 9-27, (1991).

82. I. Goykhman, B. Desiatov, J. Khurgin, J. Shappir and U. Levy, "Waveguide based compact silicon Schottky photodetector with enhanced responsivity in the telecom spectral band," Opt. Exp., Vol. 20, Issue 27, pp. 28594-28602, (2012).

83. C.-Y. Tsai, S.-P. Lu, J.-W. Lin, and P.-T. Lee, "High sensitivity plasmonic index sensor using slablike gold nanoring arrays," Appl. Phys. Lett. 98, 153108, (2011).

84. B. Desiatov, I. Goykhman, N. Mazurski, J. O. Shappir, J.B. Khurgan and U. Levy, "Plasmonic enhanced silicon pyramids for internal photoemission Schottky detectors in the near-infrared regime", Optica Lett. Vol. 2, No. 4, (2015).

85. A. Sellai and P. Dawson, "Quantum efficiency in GaAs Schottky photodetectors with enhancement due to surface plasmon excitations", Solid-State Electr. 46, 29-33 (2002)

86. S. Hassan, E. Lisicka-Skrzek, A. Olivieri, R.N. Tait and Pierre Berini, "Fabrication of a plasmonic modulator incorporating an overlaid grating coupler," Nanotech. 25 495202, (2014).

87. J. W. Yoon, W. J. Park, K. J. Lee, S. H. Song, and R. Magnusson, “Surface-plasmon mediated total absorption of light into silicon," Opt. Express 19, 20673-20680, (2011).

88. X. G. Guo, R. Zhang, J. C. Cao, et. al. "Surface plasmon-enhanced absorption in metal grating coupled terahertz quantum well photodetectors", IEEE J. Quant. Electron., 48, 1113 (2012).

89. A. Takeda, T. Aihara, M. FukuharaY. Ishii and M. Fukuda, "Sensitivity improvement of Schottkytype plasmonic detector," Proc. SPIE 8923, Micro/Nano Materials, Devices, and Systems, 89234G (2013).

90. M. Alavirad, A. Olivieri, L. Roy and P. Berini, "High-responsivity sub-bandgap hot-hole plasmonic Schottky detectors," Opt. Express., Vol. 24, No. 20, 22544-22554 (2016).

91. J. A. Bean, A. Weeks, S. Member, G. D. Boreman, "Performance optimization of antenna-coupled $\mathrm{Au} / \mathrm{AlOx} / \mathrm{Pt}$ tunnel diode infrared detectors," IEEE J. Quantum Electron., 47, 126-135 (2011). 
92. P. Genevet, J. Lin, M. A. Kats, and F. Capasso, "Holographic detection of the orbital angular momentum of light with plasmonic photodiodes," Nature Comm. 3, 1278 (2012).

93. H. Chalabi, D. Schoen and M. L. Brongersma, Hot-Electron Photodetection with a Plasmonic Nanostripe Antenna, Nano Lett., 14 (3), pp 1374-1380 (2014).

94. K. Wu, Y. Zhan, S. Wu, J. Deng and X. Li, "Surface-plasmon enhanced photodetection at communication band based on hot electrons," J. Appl. Phys. 118, 063101 (2015).

95. M. Casalino, M. Iodice, L. Sirleto, I. Rendina and G. Coppola, “Asymmetric MSM sub-bandgap all-silicon photodetector with low dark current," Opt. Expr., Vol. 21, No. 23 (2013).

96. C. Ng, J. Cadusch, S. Dligatch, A. Roberts, T. J. Davis, P. Mulvaney, and D. E. Gomez, "Hot Carrier extraction with plasmonic broadband absorbers," ACS Nano, 10 (4), pp 4704-4711, (2016).

97. F. P. G. d. Arquer and G. Konstantatos, "Metal-insulator-semiconductor heterostructures for plasmonic hot-carrier optoelectronics," Opt. Express Vol. 23, Issue 11, pp. 14715-14723, (2015).

98. A., A. Ogino, M. Iwaki, K. Hashimoto, A. Ohnuma, F. Amano, B. Ohtani, and H. Kominami, "Gold-titanium(IV) oxide plasmonic photocatalysts prepared by a colloid-photodeposition method: correlation between physical properties and photocatalytic activities," Langmuir 28, 13105-13111 (2012).

99. X. Shi, X., K. Ueno, N. Takabayashi and H. Misawa, "Plasmon-enhanced photocurrent generation and water oxidation with a gold nanoislandloaded titanium dioxide photoelectrode," J. Phys. Chem. C $117,2494-2499$ (2013).

100. D. Gong, W. Chye J. Hoa, Y. Tanga, Q. Taya, Y. Laia, J. G. Highfieldb and Z. Chen, "Silver decorated titanate/titania nanostructures for efficient solar driven photocatalysis," J. Solid State Chem. 189, 117-122 (2012).

101. Y. Fang, Yang Jiao, K.i Xiong, R. Ogier, Z. J. Yang, S. Gao, A. B. Dahlin, and M. Käll, "Plasmon Enhanced Internal Photoemission in Antenna-Spacer-Mirror Based Au/TiO2 Nanostructures," Nano Lett., 15 (6), pp 4059-4065, (2015). 
102. Y. Shiraishi, D. Tsukamoto, Y. Sugano, A. Shiro, S. Ichikawa, S. Tanaka and T. Hirai, "Platinum nanoparticles supported on anatase titanium dioxide as highly active catalysts for aerobic oxidation under visible light irradiation," ACS Catal. 2, 1984-1992 (2012).

103. F. Wu, et al., "Photocatalytic activity of $\mathrm{Ag} / \mathrm{TiO}_{2}$ nanotube arrays enhanced by surface plasmon resonance and application in hydrogen evolution by water splitting," Plasmonics 8, 501-508 (2012).

104. H. M. Chen et al., "Plasmon inducing effects for enhanced photoelectrochemical water splitting: X-ray absorption approach to electronic structures," ACS Nano 6, 7362-7372 (2012).

105. Z. Han, Z. et al., "Visible-light photocatalytic application of hierarchical Au-ZnO flower-rod heterostructures via surface plasmon resonance," Plasmonics 8, 1193-1202 (2013).

106. E. Kazuma and T. Tatsuma, "Photoelectrochemical analysis of allowed and forbidden multipole plasmon modes of polydisperse Ag nanorods," J. Phys. Chem. C 117, 2435-3441 (2013).

107. P. Berini, "Plasmon-polariton waves guided by thin lossy metal films of finite width: Bound modes of asymmetric structures," Phys. Rev. B, 63, 125417, (2001).

108. A. Olivieri, A. Akbari and P. Berini, "Surface plasmon waveguide Schottky detectors operating near breakdown," Phys. Stat. Sol. Rap. Res. Lett., 4, 283-285 (2010).

109. A. Akbari, A. Olivieri and P. Berini, "Sub-bandgap asymmetric surface plasmon waveguide Schottky detectors on silicon,” IEEE J. Sel. Top. Quant. Electr. 19, 4600209 (2013).

110. K. T. Lin, H. L. Chen, Y. S. Lai, and C. C. Yu, "Silicon-based broadband antenna for high responsivity and polarization-insensitive photodetection at telecommunication wavelengths," Nat. Commun. 5(3288), (2013).

111. A. Ben-Moshe, B. M. Maoz, A. O. Govorov, and G. Markovich, "Chirality and chiroptical effects in inorganic nanocrystal systems with plasmon and exciton resonances," Chem. Soc. Rev. 42, 7028$7041(2013)$.

112. W. Li , Z. J. Coppens, L. V. Besteiro, W. Wang, A. O. Govorov, and J. Valentine, "Circularly polarized light detection with hot electrons in chiral plasmonic metamaterials," Nat. Commun., 6(8379), (2015). 
113. Y. Yang, R. C. da Costa, M. J. Fuchter, and A. J. Campbell, "Circularly polarized light detection by a chiral organic semiconductor transistor," Nat. Photon. 7, 634-638 (2013).

114. M. Casalino, "Internal Photoemission Theory: Comments and Theoretical Limitations on the Performance of Near-Infrared Silicon Schottky Photodetector," IEEE J. Quant. Electron. 52(4), (2016). 115. M. W. Knight, Y. Wang, A. S. Urban, A. Sobhani, B. Y. Zheng, P. Nordlander and N. J. Halas, “Embedding Plasmonic Nanostructure Diodes Enhances Hot Electron Emission," Nano Lett., 13 (4), pp 1687-1692 (2014).

116. L. Yang, P. Kou, J. Shen, E. H. Lee, S. He, "Proposal of a polarization-insensitive and highefficiency hot-carrier Schottky photodetector integrated with a silicon ridge waveguide," J. of Opt. 17(12):125010, (2015).

117 A. Tanaka et al., "Gold-titanium(IV) oxide plasmonic photocatalysts prepared by a colloidphotodeposition method: correlation between physical properties and photocatalytic activities," Langmuir 28, 13105-13111 (2012).

118. I. Goykhman, U. Sassi, B. Desiatov, N. Mazurski, S. Milana, D. de Fazio, A. Eiden, J. Khurgin, J. Shappir, U. Levy, and A. C. Ferrari, “On-Chip Integrated, Silicon-Graphene Plasmonic Schottky Photodetector with High Responsivity and Avalanche Photogain,” Nano Lett. 16(5): 3005-3013, 2016. 